\title{
Search for the neutral MSSM Higgs bosons in the $\tau \tau$ decay channels at CDF Run II
}

\author{
Cristóbal Cuenca Almenar \\ Departament de Física Atòmica, Molecular i Nuclear (Universitat de València) and \\ IFIC (CSIC - Universitat de València)
}


Memòria presentada per a l'obtenció del títol de Doctor per la Universitat de València 
Na Victoria Castillo Giménez, professora titular del departament de Física Atòmica, Molecular i Nuclear de la Universitat de València,

En John Conway, catedràtic del departament de Física de la Universitat de Califòrnia a Davis i

En Juan Valls Ferrer, científic titular del CSIC

\section{CERTIFIQUEN}

que la present memòria, search for neutral MSSM Higgs bosons in the tau tau decay channels, ha estat realitzada per En Cristóbal Cuenca Almenar sota la seua direcció al departament de Física Atòmica, Molecular i Nuclear de la Universitat de València i constitueix la seua Tesi Doctoral, que presenta en

compliment dels requisits per a l'obtenció del títol de Doctor per la Universitat de València.

Per a que així conste, signem el present certificat en Paterna, a 11 d'abril de 2008,

Victoria Castillo Giménez

John Conway

Juan Valls Ferrer 


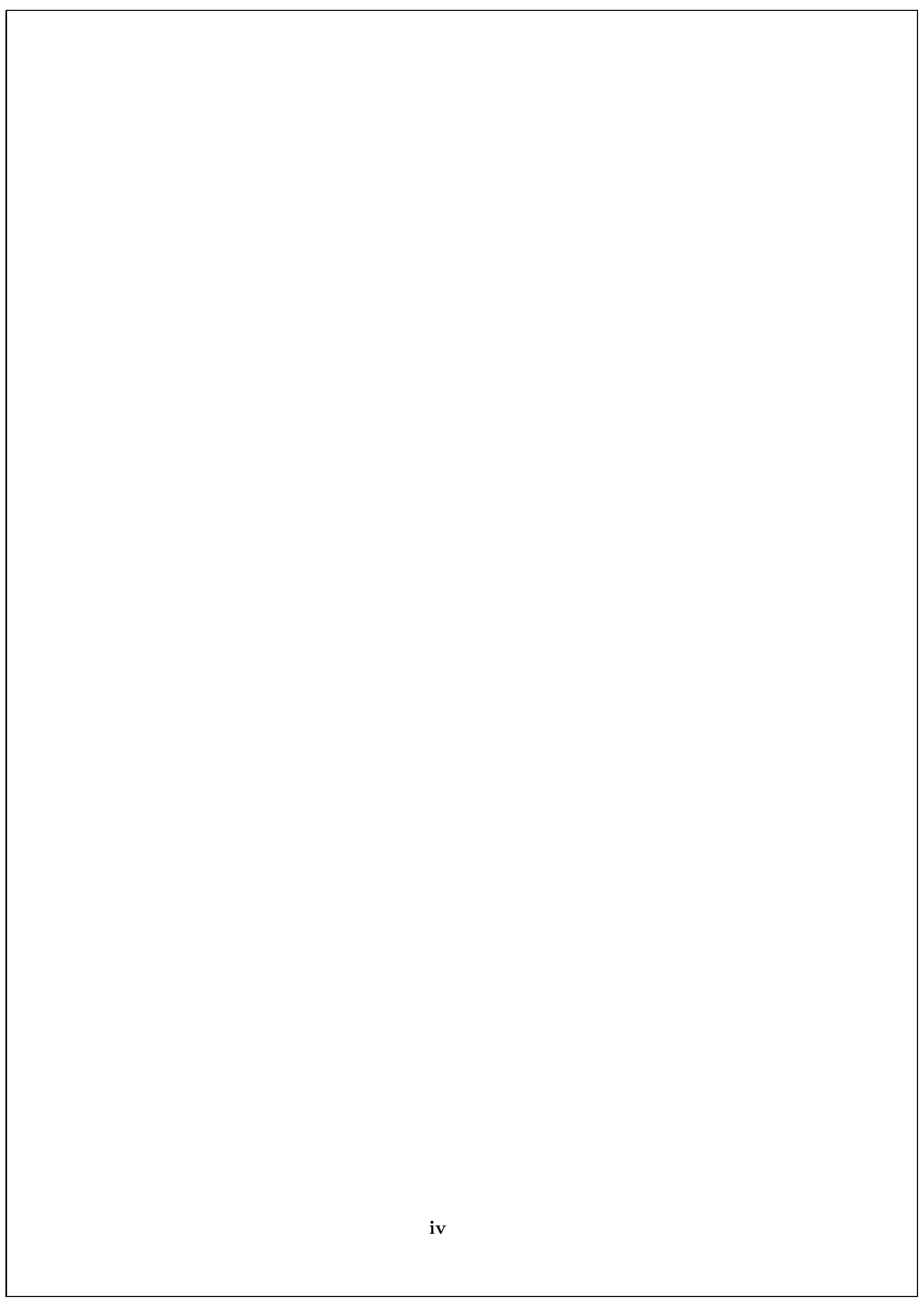




\title{
Search for the neutral MSSM Higgs bosons in the $\tau \tau$ decay channels at CDF Run II
}

\author{
Cristóbal Cuenca Almenar
}

This thesis presents the results on a search for the neutral MSSM Higgs bosons decaying to tau pairs, with least one of these taus decays leptonically. The search was performed with a sample of $1.8 \mathrm{fb}^{-1}$ of proton-antiproton collisions at $\sqrt{s}=1.96 \mathrm{TeV}$ provided by the Tevatron and collected by CDF Run II. No significant excess over the Standard Model prediction was found and a $95 \%$ confidence level exclusion limit have been set on the cross section times branching ratio as a function of the Higgs boson mass. This limit has been translated into the MSSM Higgs sector parameter plane, $\tan \beta$ vs. $M_{A}$, for the four different benchmark scenarios. 


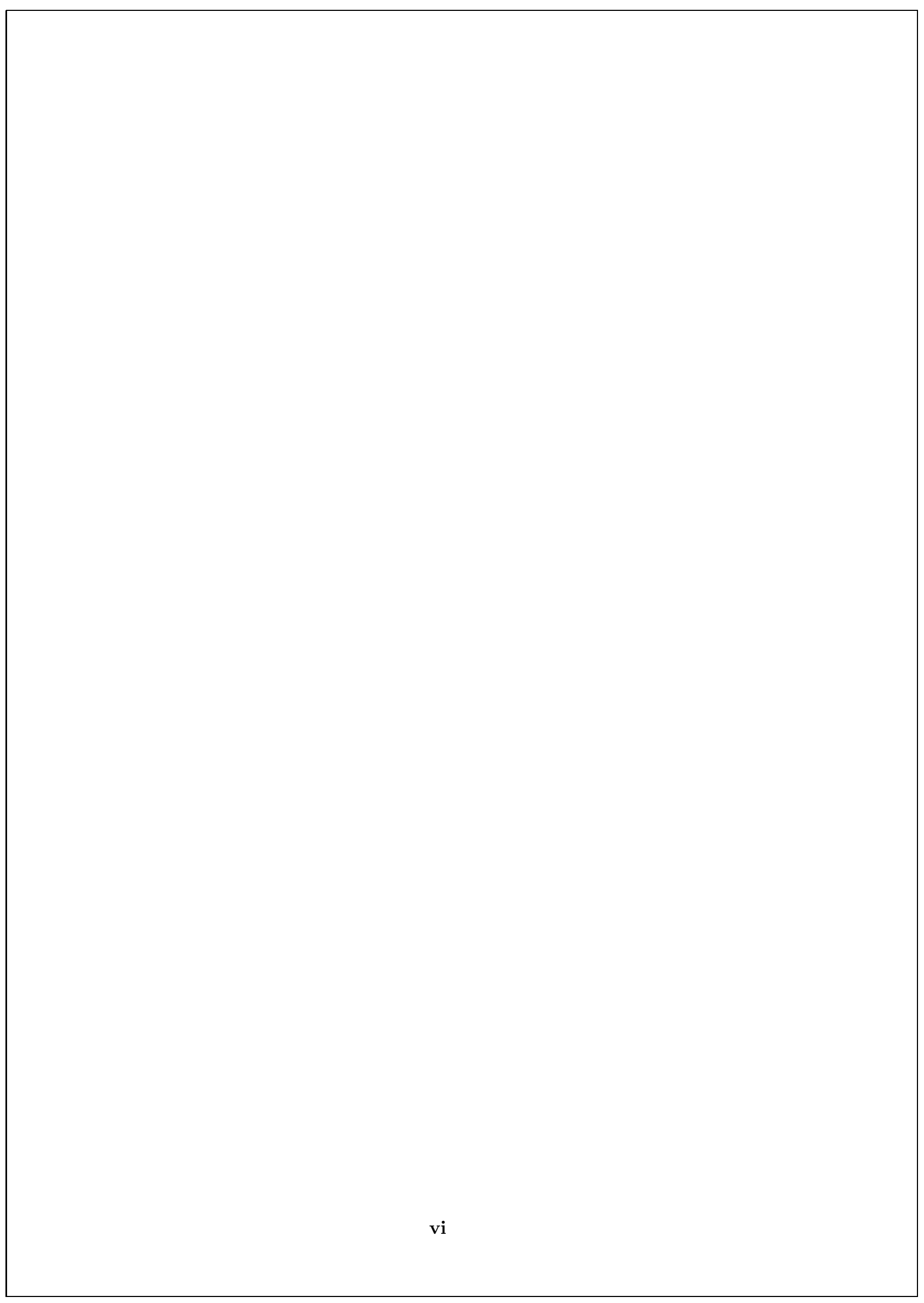




\section{Acknowledgements}

I wish to thank all the individuals and institutions involved in the successful submission of this work. Especially, I would like to thank my thesis supervisors, Victoria Castillo Giménez from the University of València, John Conway from the University of California (Davis) and Juan Valls Ferrer from IFIC (CSIC and University of València). During my years as a PhD student, many decisions had a critical impact on the final result; only now I realize how relevant their advice was.

During my last year as an undergraduate, Emilio Higón Rodríguez and Victoria backed my application for a collaboration grant that allowed the beginning of my work with the Department of Atomic, Molecular and Nuclear physics. They invited me to enroll in the CERN summer student program and, later, they welcomed me in their research group. The trust they put in me about five years ago was the seed of this thesis. I am indebted to Emilio, at that point the department head and project leader, and to Victoria, my tutor since I joined and the current department head, for their support. During this time, I also met and interacted with Antoni Ferrer Soria, national high energy program manager then and now member of my thesis committee, who always strove to convey his deep knowledge and excitement about physics. Moreover, I want to mention the support of our staff at IFIC, specially the members of IT and administration departments.

Recently graduated Belen Salvachúa and I started our PhD program at the same time. Both Esteban Fullana Torregrosa, the "senior" student of the group, and Xose Castelo, our engineer, were our guides at CERN for the first few weeks. 
Having my group colleagues at CERN definitely made everything easier. With them, I also interacted with many other students and scientists, mainly involved in TileCal construction and commissioning. I would like to mention Rupert Leitner, project leader, and Anna Henriques, CERN group leader, for their welcoming attitude. In the testbeam, we always had Bob Stanek and Stan Nemecek providing as much help as needed; Tomas Davidek was intrumental for the testbeam data analysis I presented for my Advance Studies Degree. I learned most of what I know about FPGA's and the TileCal Read out Drivers from Xose and the engineers of the University of Geneva, especially Annie Leger. Finally, implementing an optimal filter for TileCal in ATHENA wouldn't have been possible without the expertise of Esteban, Richard Teuscher and the technical help of Sasha Solodkov. All of these scientists deserve my admiration for their knowledge and my gratitude for their help.

I enjoyed many good times with the students collaborating in TileCal and other experiments. I remember vividly the students from Pisa (Iacoppo, Francesca, Andrea,...), Barcelona (Olga, Xavi, Oriol, Ernest, Míriam,...), Valencia (Jose Enrique,...), Granada (Alberto, María,...) amongst many others which made those months at CERN much nicer.

After I got my Advanced Studies Degree I joined CDF, as a visiting graduate student at UC Davis. I am thankful to Juan for this arrangement and, of course, to John Conway for welcoming me into his group and having provided this opportunity to perform data analysis. Actually, the whole group of UC Davis was nice and supportive. Sasha Baroiant, Stan Forester and Aron Soha helped me with CDF code for the first months; Robin Erbacher and Max Chertok, both faculty at Davis, were also a reference in CDF, as well as the other postdocs and students and the members of the group based at FNAL and Davis. I would like to specially thank Rob and his coffee machine, for many interesting conversations, and my office mates Andrew and Will.

The analysis presented in this thesis is framed in a long project to use tau leptons in Higgs seaches at CDF. Several individuals have been involved, out of which I would like to warmly thank John, Anton Anastassov and Dongwook Jang. Anton developed many techniques, owns a profound expertise in tau reconstruction and a fine feeling for the physics of the Tevatron while Dongwook is behind several 
implementations and studies. Endless discussions with Anton and Dongwook were instrumental for this result. This thesis wouldn't have been possible without their work and collaboration. Moreover, I have to thank John for sharing his endless know-how on template fitting and limit extraction and Amit Lath for his insight in MSSM interpretation. If I now feel more comfortable relaying on PYTHIA is partially due to Steve Mrenna's code fixes after our studies.

Being based in the CDF trailers, I had the opportunity to collaborate with many brilliant physicists. Many conversations with them helped me deepen my understanding of the physics, the detector and technical issues of CDF, the different analysis tools and the limitations of our measurements. I specially want to thank Óscar González for sharing his knwledge on QCD and Ray Culbertson and Pasha Murat for his invaluable help with stntuple and, in particular, with the cataloguing effort. Also, the months I spent in the control room taking data were done much nicer by Dee Hanh, Steve, J.J. and the whole Quality of Life Committee.

My life in the past three years would have been extremely quiet if I hadn't related to the Spanish cluster at FNAL and our friends in the city, without them I wouldn't have started playing volleyball or mus. I would like to thank Olga, Xavi and Oriol, for their welcome; Kike for that sincere friendship we shared in so many trips; the "new" students Bruno, Miguel, Bárbara and Carol for so many good times; also, I don't want to forget our good friends from the other side of the ring, Arán and Ernest. I would like to mention Susana for provinding shelter when I first moved to Illinois, and Olga and Michel for their intense and lasting care. I'm especially moved by the care I felt when I was trying to finish up the analysis: I was invited over for dinner so many times! Finally, I can't forget the fishbowl; I still smile when I remember all we lived together in Chicago and surroundings.

Becoming an elected GSA officer and UEC member also added precious experiences to my stay at FNAL. I want to tank my fellow GSA officers for all their work, Leah, Jim, Alexis and Tuula. Specially our trip to DC was a very intense learning experience: FNAL and SLAC representing the field in "the hill".

Overall, I spent about three and a half years in the Chicago suburbs. I am deeply thankful to those that made this time better, particularly to my house mate Ankush Mitra, always so patient with the cluster. Across the sea, my family and friends had to deal with my lack of skills to keep long-distance relationships. 
Not only they were always anchor and support to me but also they never hesitated in helping in any circumstance. Afterall, having to miss most of the events and celebrations you lived in Valencia during this years was the most difficult part.

To all of you, and specially to my parents, Salvador and Encarna, and brothers, Salva, Joan and Vicente, my deepest and most sincere acknowledgment and gratitude. 
A la meua madrina, la perla fina d'Alboraia, acceptant sense entendre el començament d'un projecte que ha vist acabat transgredida la història 


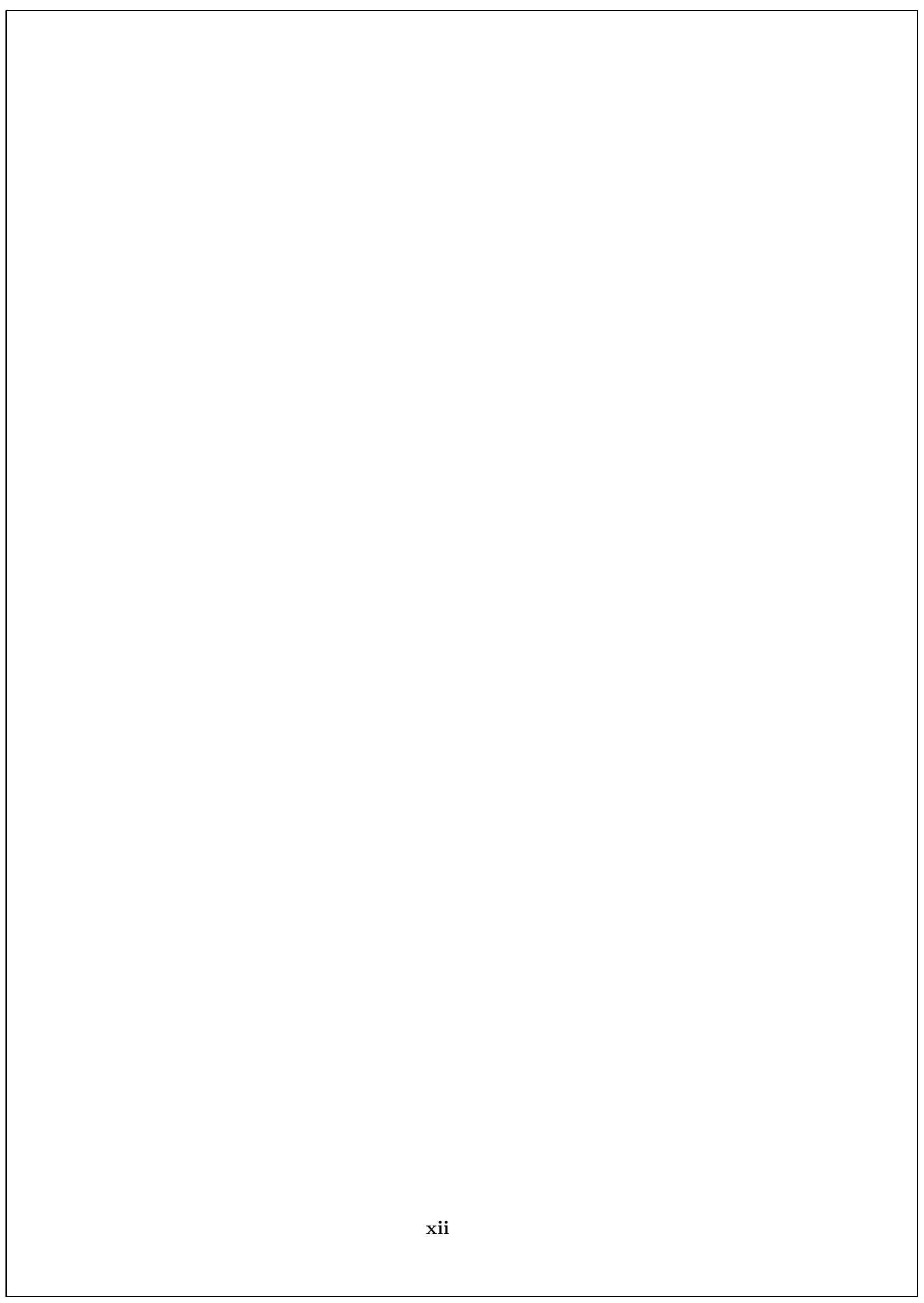




\section{Contents}

1 Introduction 1

2 Theory Introduction $\quad 5$

2.1 Standard Model . . . . . . . . . . . . . . . . . . 6

2.1 .1 Quantum Electrodynamics . . . . . . . . . 8

2.1.2 Quantum Chromodynamics . . . . . . . . . . . . . 9

2.1.2.1 Hadron-hadron processes and QCD Factorization 11

2.1.2.2 Parton Distribution Functions _. . . . . . . . 12

2.1.2.3 PDFs uncertainties $\ldots \ldots \ldots \ldots \ldots$

2.1.3 Electroweak Theory . . . . . . . . . . . . . . . . . 14

2.1.4 The Higgs mechanism . . . . . . . . . . . . . . . . 17

2.1.5 Standard Model Limitations . . . . . . . . . . . . . . . . . 21

2.2 Minimal Supersymmetric Extension of the Standard Model . . . . 23

2.2 .1 Supersymmetry . . . . . . . . . . . . . . 23

2.2.2 Supersymmetry and the hierarchy problem . . . . . . . 24

2.2.3 Other benefits from the introduction of SUSY . . . . . . . 24 
2.2.4 The Minimal Supersymmetric Standard Model . . . . . . . 26

2.2.4.1 MSSM Lagrangian and R-parity . . . . . . . . 30

2.2 .5 MSSM Higgs Sector $\ldots \ldots \ldots \ldots$

3 Experimental setup $\quad 39$

3.1 The Tevatron Collider . . . . . . . . . . . . . . . . . . . . 39

3.2 CDF Run II detector . . . . . . . . . . . . . . . . . . . 43

3.2.1 Tracking and Time of Flight systems . . . . . . . . . . 45

3.2 .2 Calorimeter system . . . . . . . . . . . . . . . 47

3.2.2.1 Central Calorimeters . . . . . . . . . 48

3.2.2.2 Shower Maximum Detector . . . . . . . . . . 50

3.2.2.3 Plug Calorimeters . . . . . . . . . . . . . . 51

3.2 .3 Muons system . . . . . . . . . . . . . . . . . 52

3.3 Luminosity Measurement . . . . . . . . . . . . . . . . . 54

$3.3 .1 \quad$ CLC detector . . . . . . . . . . . . . . . . . 54

3.3.2 Measurement of the luminosity . . . . . . . . . . 55

3.4 Trigger and Data Acquisition . . . . . . . . . . . . . 57

3.4 .1 Level 1 trigger . . . . . . . . . . . . . . . . . 59

3.4 .2 Level 2 trigger . . . . . . . . . . . . . . . 60

3.4 .3 Level 3 trigger . . . . . . . . . . . . . . . . 63

4 Data Samples and Simulation $\quad 65$

4.1 Data . . . . . . . . . . . . . . . . . 66

4.2 Monte Carlo . . . . . . . . . . . . . . . . . . . . . 67

xiv 
$\begin{array}{llr}5 & \text { Trigger efficiency } & 69\end{array}$

$5.1 \quad$ Introduction . . . . . . . . . . . . . . . . . 69

5.2 Trigger paths description $\ldots \ldots \ldots \ldots$. . . . . . . . . . 69

5.2 .1 Level 2 trigger requirements . . . . . . . . . . . . 70

5.2 .2 Level 3 trigger requirements . . . . . . . . . . . . . . 71

5.3 Data Samples and event selection . . . . . . . . . . . . . 72

5.4 Level 2 trigger efficiency . . . . . . . . . . . . . . . 72

5.5 Level 3 trigger efficiency . . . . . . . . . . . . . . . . . 75

6 Particle identification $\quad 85$

6.1 Electrons .......................... 86

6.2 Muons . . . . . . . . . . . . . . . . . . . . 87

6.3 Taus . . . . . . . . . . . . . . . . . . . 90

6.4 Missing transverse energy . . . . . . . . . . . . . . . . . . 96

7 Event Selection and Background Estimation $\quad 99$

7.1 Fake Rate Measurement and Consistency Checks . . . . . . . . . . 99

7.2 Event cuts . . . . . . . . . . . . . . . . . . 105

7.2 .1 General requirements . . . . . . . . . . . . 106

$7.2 .2 \quad \zeta$ cut . . . . . . . . . . . . 106

7.2.3 Suppression of QCD backgrounds. . . . . . . . . . . . 107

$7.2 .4 \quad Z \rightarrow \mu \mu$ removal . . . . . . . . . . . . . . . 109

7.3 Backgrounds and estimation methods . . . . . . . . . . . 110

$7.3 .1 \quad Z \rightarrow l l \ldots \ldots \ldots \ldots \ldots \ldots \ldots$ 
7.3.2 Di-boson, $W+\gamma$, and $t \bar{t} \ldots \ldots \ldots \ldots \ldots$

7.3.3 Backgrounds with misidentified or non-isolated $e$ or $\mu$ in the $\tau_{e} \tau_{\mu}$ detection mode . . . . . . . . . . . . 110

7.3.4 Backgrounds from misidentified $j e t \rightarrow \tau_{\text {had }}$ in the $\tau_{l} \tau_{\text {had }}$ detection modes . . . . . . . . . . . . . . . . 112

7.4 Signal selection efficiency $\ldots \ldots \ldots \ldots$. . . . . . . . . 115

$\begin{array}{lll}8 & \text { Results } & 117\end{array}$

8.1 Observed Events . . . . . . . . . . . . . . . . . . . 117

8.2 Systematic uncertainties . . . . . . . . . . . . . . . . 118

8.3 Fitting Method . . . . . . . . . . . . . . . . . 130

8.4 Nuisance Parameters for Systematic Uncertainties . . . . . . . . 132

8.4.1 Template Morphing . . . . . . . . . . . . . . . 133

8.4.2 Profile Likelihood . . . . . . . . . . . . . . . . . 133

8.5 Expected Sensitivity . . . . . . . . . . . . . . . . . . . 134

8.6 Results . . . . . . . . . . . . . . . . . . . . . . . 134

8.7 Interpretation of the Limits . . . . . . . . . . . . . 138

8.7.1 Production Cross-section Calculations . . . . . . . . . . 139

8.7.2 Excluded region in $\tan \beta$ vs $m_{A} \ldots \ldots \ldots \ldots$

8.8 Full di-tau mass reconstruction using MET projection. . . . . . . . 140

9 Conclusions

$\begin{array}{ll}\text { A Resum } & 147\end{array}$

A.1 Introducció teòrica . . . . . . . . . . . . . . . . . . . . . . . . . . 147 
A.2 Dispositiu Experimental . . . . . . . . . . . . . . . . 149

A.3 Mostra de dades i trigger f . . . . . . . . . . . . . . . 151

A.4 Identificació de partícules i selecció de succesos . . . . . . . . . 151

A.5 Resultats . . . . . . . . . . . . . . . . 153

A.6 Conclusions . . . . . . . . . . . . . . . . . . . . . 154

$\begin{array}{ll}\text { Bibliography } & 155\end{array}$

$\begin{array}{ll}\text { List of Figures } & 165\end{array}$

$\begin{array}{ll}\text { List of Tables } & 171\end{array}$

$\begin{array}{ll}\text { Glossary } & 173\end{array}$

xvii 


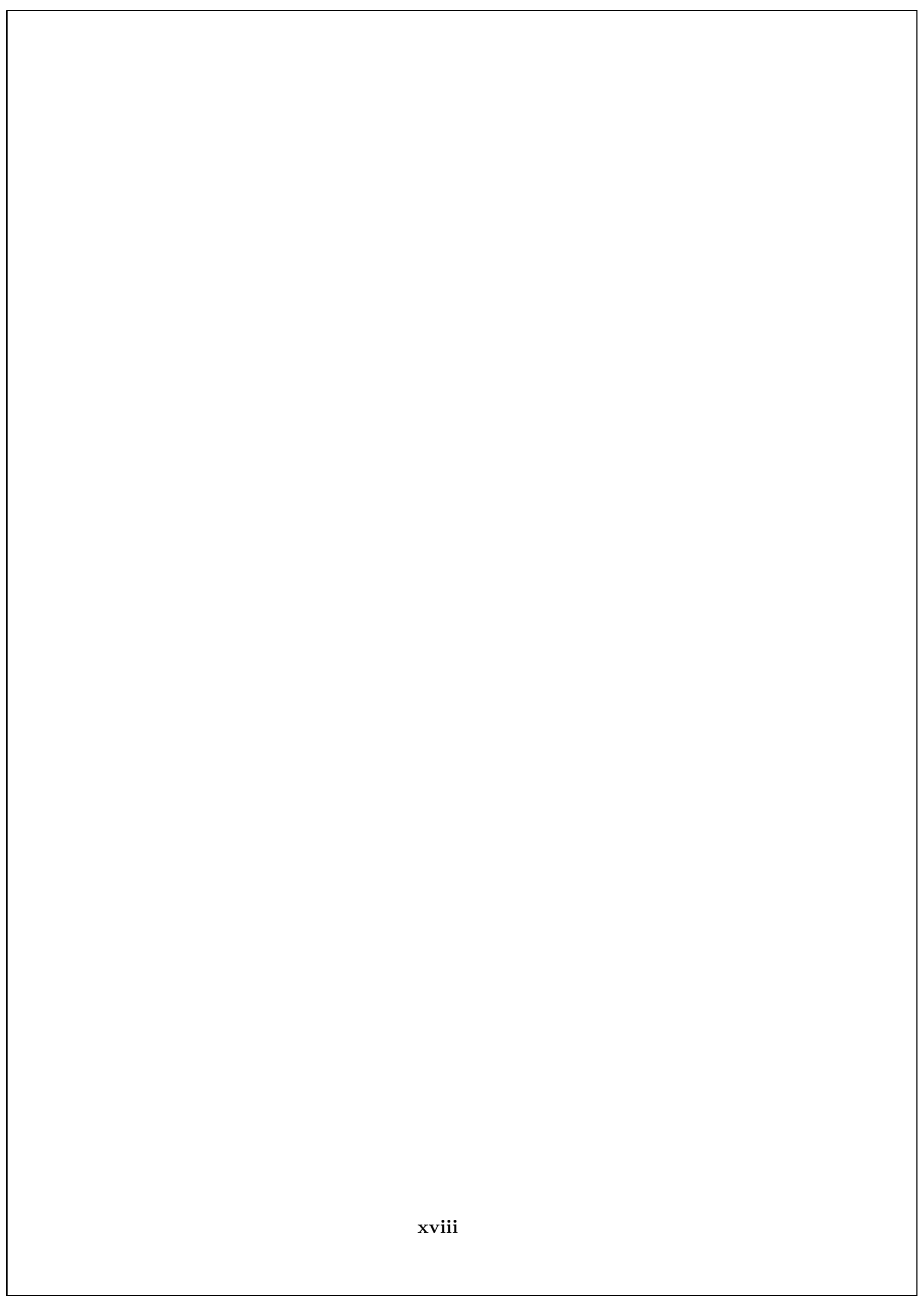


"The right to search for truth implies also a duty; one must not conceal any part of what one has recognized to be true."

- Albert Einstein

xix 


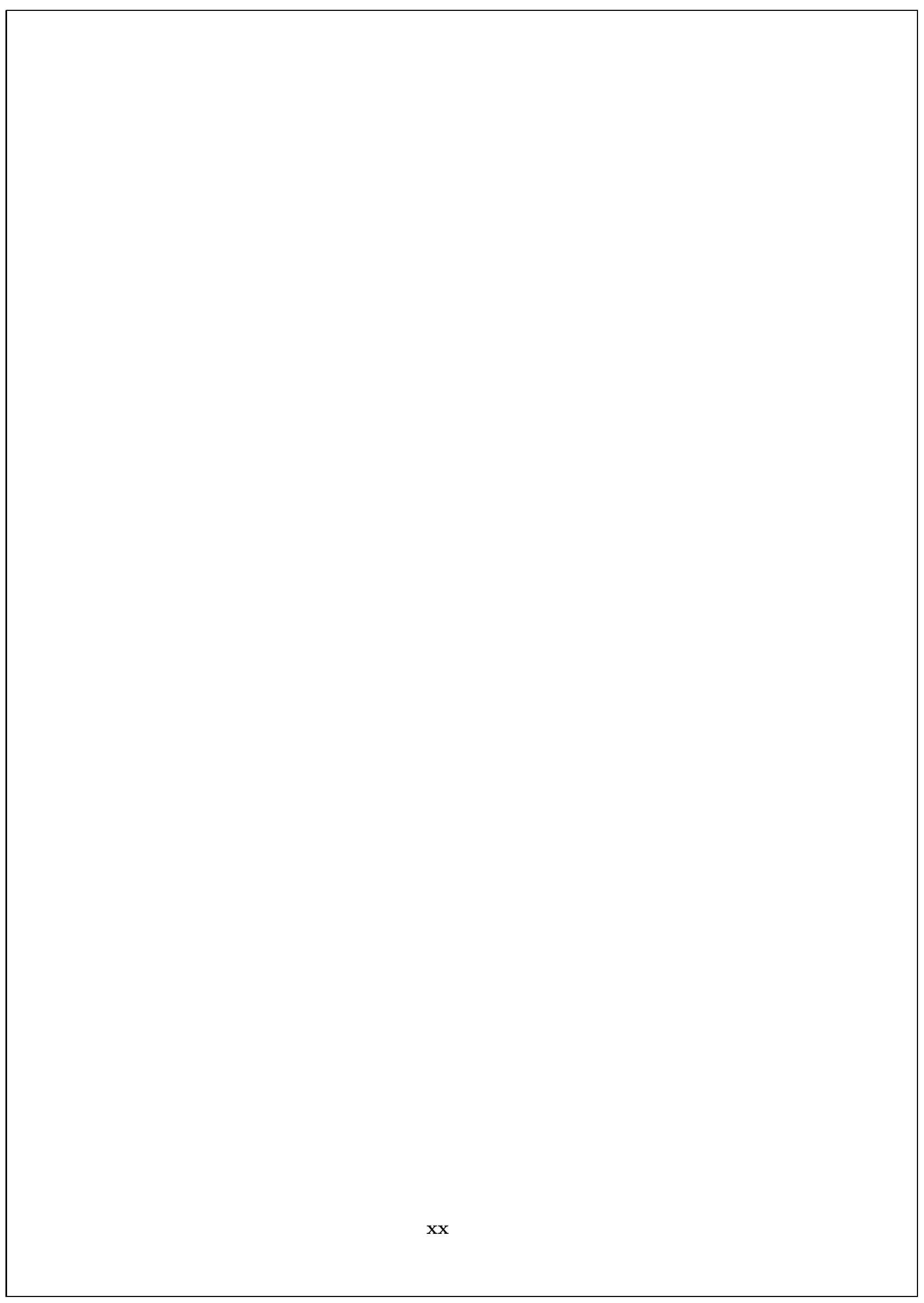




\section{Chapter 1}

\section{Introduction}

The Standard Model of particle physics has proven to be one of the most accurate descriptions of nature ever devised by human beings. The level of accuracy of some theoretical predictions has never been attained before. It includes the electromagnetic interaction, and the weak and strong force, developing the Lagrangian from symmetry principles.

There are two different types of fundamental constituents of nature, in the framework of the Standard Model: bosons and fermions. Bosons are those particles in responsible for carrying the interactions among the fermions, which constitute matter. Fermions are divide into six quarks and six leptons, forming a three-folded structure. All these fermions have an antimatter partner.

However, several difficulties point along with the idea that the Standard Model is only an effective low energy theory. These limitations include the difficulty to incorporate gravity and the lack of justification to fine tuning of some perturbative corrections. Moreover, some regions of the theory are not understood, like the mass spectrum of the Standard Model or the mechanism for electroweak symmetry breaking.

Supersymmetry is a newer theoretical framework, thought to overcome the problems found with the Standard Model, while preserving all its predictive power. It introduces a new symmetry that relates a new boson to each SM fermion and 
a new fermion to each SM boson. The model with a minimal supersymmetric content in the Higgs sector is know as Minimal Supersymmetric Extension of the Standard Model, MSSM. This theory predicts new neutral Higgs boson states that would manifest in the Tevatron.

The Tevatron is a hadron collider operating at Fermilab, USA. This accelerator provides proton-antiproton $(\mathrm{p}-\overline{\mathrm{p}})$ collisions with a center of mass energy of $\sqrt{s}=$ $1.96 \mathrm{TeV}$, the current energy frontier. $\mathrm{CDF}$ and $\mathrm{D} \varnothing$ are the detectors built to analyse products of the collisions provided by the Tevatron. Both experiments have produced a very significant scientific output in the last few years, like the discovery of the top quark or the measurement of the $\mathrm{B}_{\mathrm{s}}^{0}$ mixing. The Tevatron experiments are also reaching sensitivity to the SM Higgs boson.

The scientific reach of CDF also includes a very active program on searches for physics signatures beyond the Standard Model. These searches are, in general, signature based and share a common strategy based on two steps: describe the expected Standard Model contributions with the chosen signature and asses any deviation in the data collected by the experiment. Assuming a process in a given model, a favored final state is chosen. Then, analysts have to prove that the contributions of the Standard Model are under control, which involves understanding the performance/misperformance of the detector and considering all the different processes that might have an impact.

The analysis presented in this dissertation focuses on the search for neutral MSSM Higgs bosons decaying to tau pairs. The production of this particles could be highly enhanced at the Tevatron, turning CDF in a convenient probe to either make a discovery of new physics or limit the parameter space available in the theory. The decay channel is chosen to be di-tau to reduce the Standard Model contributions, however, the reconstruction and identification of tau leptons in CDF increases the complexity to the search. This is due to the fact that taus decay to other particles short after being produced, $\sim 35 \%$ of the tau decays are to other leptons, i.e. electrons or muons and two neutrinos, while a $\sim 65 \%$ of the decays are semi-hadronic, i.e. to charged and neutral mesons and a neutrino.

Among all the combinations of tau decays, this analysis includes three modes. One of the taus is always required to decay leptonically, to electron or muon, while the second tau is required to decay hadronically or to a different species of lepton. In 
summary, the event selection demands an electron and a hadronic tau, a muon and a hadronic tau or a muon and an electron.

The signal extraction method chosen was a profile likelihood fit. No signal is found over the predicted Standard Model background. Instead, 95\% confidence level limits are set on the production cross section times branching ratio, and then translated into the parameter space of MSSM, in four different scenarios.

This thesis sketches the basic theory concepts of the Standard Model and the Minimal Supersymmetric Extension in Chapter 2. The next section, Chapter 3, describes the Tevatron and CDF. The development of the analysis is shown in Chapter 4 to Chapter 7. Finally, Chapter 8 and Chapter 9 are devoted to discuss the results and conclusions of this work, and future prospects. 


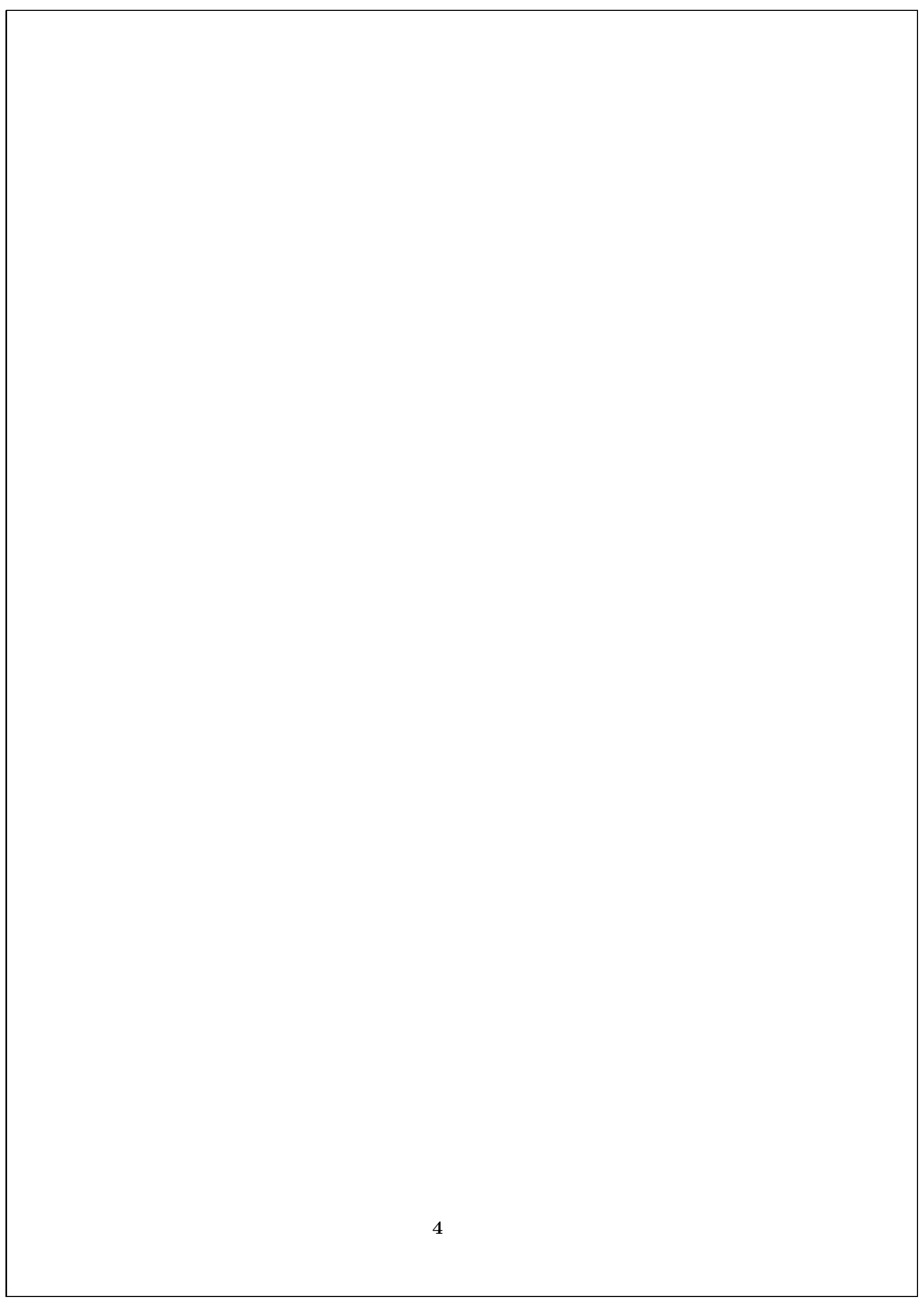




\section{Chapter 2}

\section{The Standard Model and the Minimal Supersymmetric Extension}

This chapter sketches a brief introduction to the Standard Model (SM) and to the Minimal Supersymmetric extension of the Standard Model (MSSM). The Higgs sector of the MSSM is described in a more detailed manner, as well as the experimental signature that motivates this thesis.

Although the SM has shown and excellent level of accomplishment in describing the building blocks and basic interactions of matter, several limitations of this model would indicate that it is a low energy effective theory. Some extensions of the theory, one of them being the MSSM, try to address and fix the issues that are not dealt satisfactorily in the SM. 


\subsection{Standard Model}

The Standard Model is a quantum field theory that has proven to describe to an unprecedented level of precision many experimental results [1]. A complete description of the theory can be easily found in the scientific literature $[2,3]$.

Based on several group symmetries, the SM includes the electromagnetic, weak and strong interaction. The building blocks of nature, according to the SM, are a close set of fermions and bosons. The fermions are responsible for matter, while the bosons mediate interactions.

The fermionic sector ensembles six quarks and six leptons and their antiparticles, divided in three parallel families, Figure 2.1. The members of these families are identical in every observable, except for the mass. Our most immediate world is made with the particles of the first family: the up quark (u) and down quark (d) that form the protons and neutrons in nuclei and the electrons $\left(\mathrm{e}^{-}\right)$and its associated neutrino $\left(\nu_{\mathrm{e}}\right)$, Table 2.1. The particles in the other two families are more massive and decay rapidly to the ones of the first family.

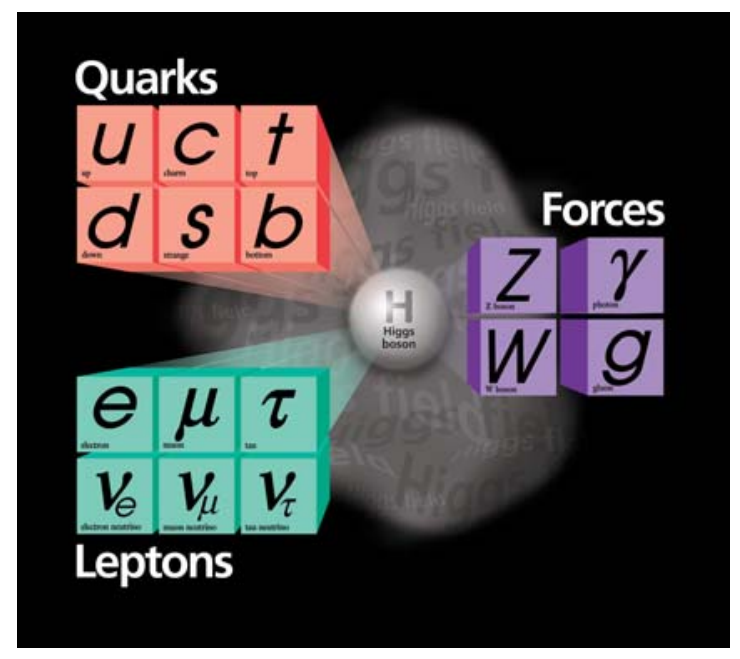

Figure 2.1: Elementary particles in the Standard Model. (Image courtesy of Fermilab Visual Media Services)

The interactions of the fermions in Table 2.1 are mediated by the bosonic con- 


\begin{tabular}{c|c|c|c}
\hline \hline & $1^{\text {st }}$ Generation & $2^{\text {nd }}$ Generation & $3^{\text {rd }}$ Generation \\
\hline \multirow{4}{*}{ quarks } & Up $(\mathbf{u})$ & Charm $(\mathbf{c})$ & Top $(\mathbf{t})$ \\
& $1.5-3.0 \mathrm{MeV} / c^{2}$ & $1.25 \pm 0.09 \mathrm{GeV} / c^{2}$ & $170.9 \pm 1.8 \mathrm{GeV} / c^{2}$ \\
\cline { 2 - 4 } & Down $(\mathbf{d})$ & Strange $(\mathbf{s})$ & Bottom $(\mathbf{b})$ \\
& $3.0-7.0 \mathrm{MeV} / c^{2}$ & $95 \pm 25 \mathrm{MeV} / c^{2}$ & $4.20 \pm 0.07 \mathrm{GeV} / c^{2}$ \\
\hline \multirow{4}{*}{ leptons } & Electron neutrino $\left(\boldsymbol{\nu}_{\mathbf{e}}\right)$ & Muon neutrino $\left(\boldsymbol{\nu}_{\boldsymbol{\mu}}\right)$ & Tau neutrino $\left(\boldsymbol{\nu}_{\boldsymbol{\tau}}\right)$ \\
& $<2 \mathrm{eV} / c^{2}$ & $<0.19 \mathrm{MeV} / c^{2}$ & $<18.2 \mathrm{MeV} / c^{2}$ \\
\cline { 2 - 4 } & Electron $(\mathbf{e})$ & Muon $(\boldsymbol{\mu})$ & Tau $(\boldsymbol{\tau})$ \\
& $0.511 \mathrm{MeV} / c^{2}$ & $105.66 \mathrm{MeV} / c^{2}$ & $1776.99_{-0.26}^{+0.29 \mathrm{MeV} / c^{2}}$ \\
\hline \hline
\end{tabular}

Table 2.1: The fermion sector of the SM. All masses are taken from the Particle Data Group (PDG) [4], except for the top quark mass, where the last TeVatron combination is quoted in [5].

stituents of the Standard Model. These bosons carry the fundamental forces derived from the symmetries, as summarized in Table 2.2. The overall symmetry of the Standard Model is the combination of the color symmetry group for the strong force $\mathrm{SU}(3)_{C}$, weak-isospin symmetry for the weak interaction of left handed particles $\mathrm{SU}(2)_{L}$ and hypercharge symmetry $\mathrm{U}(1)_{Y}$, expressed as $\mathrm{SU}(3)_{C} \times \mathrm{SU}(2)_{L} \times \mathrm{U}(1)_{Y}$. However, the original symmetry is broken in our universe, as will be shown latter.

Even if gravity is the interaction that has been known for the longest time and is the closest to our every day life experience, it still has not been successfully included in the Standard Model framework. This is one of the main arguments against the Standard Model being the theory of everything, therefore suggesting that there needs to be a somewhat more general theory. This new theory would have to include all the symmetries of the Standard Model, and, simultaneously accept a forth interaction.

In the following sections, an introduction to the different parts of the Standard Model is presented. After a brief explanation of the symmetry originating each interaction, a short discussion of the couplings and eigen states will be shown. 


\begin{tabular}{c|c|c}
\hline \hline Interaction & Particle & Mass \\
\hline electromagnetic & photon, $\gamma$ & 0.0 \\
\hline strong & gluon, $\mathbf{g}$ & 0.0 \\
\hline \multirow{2}{*}{ weak } & $\mathrm{W}^{ \pm}$ & $80.403 \pm 0.029 \mathrm{GeV} / c^{2}$ \\
\cline { 2 - 3 } & $\mathbf{Z}^{\mathbf{0}}$ & $91.188 \pm 0.002 \mathrm{GeV} / c^{2}$ \\
\hline \hline
\end{tabular}

Table 2.2: The gauge bosons of the Standard Model and their interactions [5].

\subsubsection{Quantum Electrodynamics}

Quantum Electrodynamics (QED) was developed in the late 1940s and early 1950s chiefly by Feynman, Schwinger and Tomonaga [6], describing electromagnetic interactions of electrons and photons. This is a quantum relativistic renormalizable theory which is invariant under a change of phase or gauge, $\theta$ :

$$
\psi \rightarrow \psi^{\prime}=e^{i Q \theta} \psi
$$

where $Q$ represents the charge and $\psi$ is the Dirac field (spin 1/2). In order to promote the global symmetry under $\mathrm{U}(1)$ transformations, responsible for the conservation of the charge, to a local one $(\theta=\theta(x))$, the covariant derivative needs to be introduced:

$$
D_{\mu} \equiv \partial_{\mu}-i e Q A_{\mu}
$$

where $A_{\mu}$ is a field that satisfies:

$$
A_{\mu} \rightarrow A_{\mu}^{\prime} \equiv A_{\mu}+\frac{1}{e} \partial_{\mu} \theta
$$

Therefore, the Lagrangian describing the theory becomes:

$$
\mathcal{L}=\bar{\psi}\left(i \gamma^{\mu} D_{\mu}-m\right) \psi=\bar{\psi}\left(i \gamma^{\mu} \partial_{\mu}-m\right) \psi+\mathcal{L}_{I}
$$

where the last term corresponds to the interaction with the new field, $A_{\mu}$ :

$$
\mathcal{L}_{I}=e Q A_{\mu}\left(\bar{\psi} \gamma^{\mu} \psi\right)
$$


In addition, the kinetic energy of the new field needs to be introduced. From Maxwell's equations, the kinetic term must be of the form:

$$
\mathcal{L}_{K}=-\frac{1}{4} F_{\mu \nu} F^{\mu \nu}
$$

where $F_{\mu \nu} \equiv \partial_{\mu} A_{\nu}-\partial_{\nu} A_{\mu}$.

Thus, in this theory the electromagnetic interaction is described by two quantum fields: one for the charged particles and one for the photon. The strength of the interaction is usually described by the coupling constant $\alpha_{\mathrm{e} m}$ whose value depends on the momentum transfer $q^{2}$ in an interaction. At $q^{2} \rightarrow 0$ (or low energies) the coupling constant value is that of the fine structure constant, $\alpha_{\mathrm{e} m}=\frac{e^{2}}{4 \pi / h c}=\frac{1}{137}$. At the scale of the $Z$-boson (short distances), its value increases: $\alpha_{\mathrm{e} m}\left(m_{Z}\right) \approx \frac{1}{128}$.

\subsubsection{Quantum Chromodynamics}

One of the cornerstones of the Standard Model is Quantum Chromodynamics (QCD) that describes the strong interaction. Following the way opened by QED and Yang-Mills theories, QCD was developed in 1973 [7] in the context of Quantum Field Theory based in $\mathrm{SU}(3)$ symmetry group [8]. It is a non-abelian theory and the Lagrangian, that describes the strong interaction of colored quarks and gluons ${ }^{1}$, is given by:

$$
\mathcal{L}_{Q C D}=\sum_{\text {flavor }} \overline{q_{a}}\left(i \gamma^{\mu} D_{\mu}-m_{q}\right)_{a b} q_{b}-\frac{1}{4} F_{\alpha \beta}^{A} F_{A}^{\alpha \beta}
$$

where the sum runs over the six different flavors of the quarks. $F_{\alpha \beta}^{A}$ is the field strength tensor derived from the gluon field $A_{\alpha}^{A}$ as,

$$
F_{\alpha \beta}^{A}=\left[\partial_{\alpha} A_{\beta}^{A}-\partial_{\beta} A_{\alpha}^{A}-g f^{A B C} A_{\alpha}^{B} A_{\beta}^{C}\right]
$$

and the indices A,B,C run over the eight color degrees of freedom of gluon field. $\mathrm{g}$ is the coupling constant, which determines the strength of the interaction between colored quanta, and $f^{A B C}$ are the structure constants of the $\mathrm{SU}(3)$ color group.

\footnotetext{
${ }^{1}$ The charge associated with the strong interaction is the color-charge. The color property was introduced to quarks satisfied the requirement of Pauli exclusion principle. Posterior experiment results proved the validity of color hypothesis
} 
The third term in equation 2.8 shows the non-abelian nature of QCD. This term describes the property of interaction between gluons, resulting in the very different behavior of the strong interaction compared to the electromagnetic interaction. This self-coupling is the reason for the strong coupling constant, $\alpha_{s}=\frac{g^{2}}{4 \pi}$, is large at small energies (large distances) and decreases at high energies (small distance) as is shown in figure 2.2 .

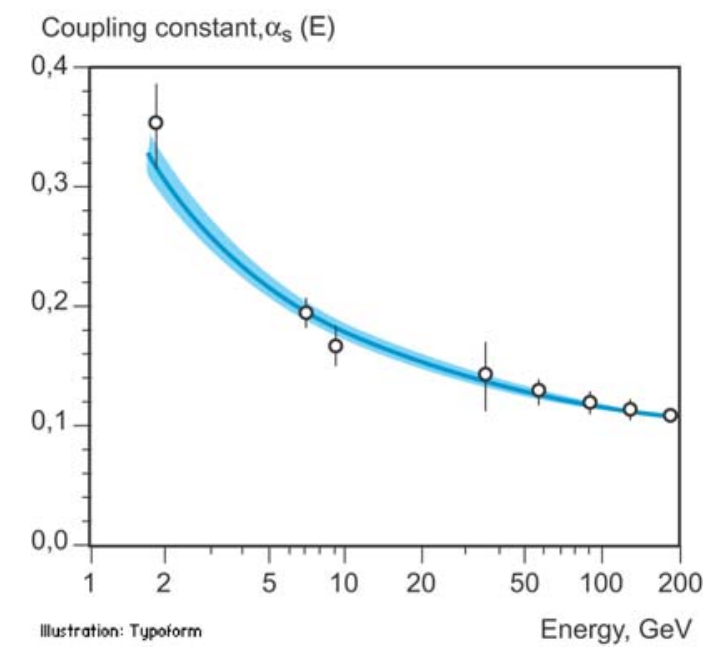

Figure 2.2: The value of the running coupling constant, $\alpha_{S}$, as a function of the energy scale E.

This characteristic running of $\alpha_{S}$ is used to explain the observed behavior of the strong interaction:

- Asymptotic freedom: At high energies (small distance) the strong interaction proceeds via color field of reduced strength and the quarks and gluons behave as essentially free, non-interacting particles.

- Confinement: At low energies (or large distance) the strength of the color field is increasing, since the potential behaves as $V(r) \sim \lambda r$, and in this way the quarks and gluons can never be observed as free particles. If two interacting partons are separated, the energy of the field increases so much that it creates new interacting particles and at the end it is left with colorless hadrons containing the partons. Therefore partons are not observed as free particles. 
It is important to note that the asymptotic freedom property allows the application of perturbation theory to calculate cross section measurements in scattering processes where quarks and gluons are involved. Moreover, this property explains the partial success of the naive Quark Parton Model approach, which is going to be presented below.

\subsubsection{Hadron-hadron processes and QCD Factorization}

An essential ingredient in the description of the DIS above is the concept of QCD Factorization, which allows us to extract universal PDFs that can be employed in other hadron processes. The QCD Factorization theorem stays that a given event can be factorized into short- and long-distance related parts, where the long-distance physics is included in the PDFs.

The cross section for a hard scattering process initiated by two hadrons with fourmomenta $P_{1}$ and $P_{2}$ can be written as:

$$
\sigma\left(P_{1}, P_{2}\right)=\sum_{i, j} \int d x_{1} d x_{2} f_{i}\left(x_{1}, \mu_{F}^{2}\right) f_{j}\left(x_{2}, \mu_{F}^{2}\right) \hat{\sigma}_{i j}\left(p_{1}, p_{2}, \alpha_{s}\left(\mu_{F}^{2}\right), Q^{2} / \mu_{F}^{2}\right),
$$

where the momenta of the partons which participate in the hard interaction are $p_{1}=x_{1} P_{1}$ and $p_{2}=x_{2} P_{2}$. The $\hat{\sigma}_{i j}$ is the parton-parton cross section and $f_{i}\left(x_{1}, \mu_{F}^{2}\right)$ are the well-known PDFs defined at a factorization scale, $\mu_{F}$. This factorization scale $\mu_{F}$ is an arbitrary parameter. It can be thought of as the scale which separates the soft and the hard processes. Thus a parton emitted with small transverse momentum less than the scale $\mu_{F}$ is considered part of the hadron structure and is absorbed into the parton distribution. Processes with transverse momentum larger than $\mu_{F}$ is part of the parton-parton cross section. The scale $\mu_{F}$ is typically chosen to be of the order of the hard scale $\mathrm{Q}$, which characterizes the parton-parton interaction. Principally, any observable should be invariant under variations of this scale. This is formally expressed as:

$$
\mu^{2} \frac{d}{d \mu^{2}} \Gamma=0
$$

where $\Gamma$ is the observable we are interested in. In the perturbative approach, this 
equation has to be applied to the perturbative expansion of the observable,

$$
\Gamma=\Gamma_{0}+\alpha_{s} \Gamma_{1}+\alpha_{s}^{2} \Gamma_{2}+\ldots
$$

therefore, the equation transforms into

$$
\mu^{2} \frac{d}{d \mu^{2}} \sum_{j=0}^{N} \alpha_{s}^{j} \Gamma_{j}=\mathcal{O}\left(\alpha_{s}^{N+1}\right),
$$

showing that the variation of the observable with the scale is given by terms which were not included in the perturbative expansion. The more terms included in the perturbative expansion, the weaker the dependence on $\mu$ will be.

Similar to QED, different types of divergences appear in the pQCD calculations. The renormalization is the standard regularization procedure used to solve these divergences [9]. The procedure is not unique, i.e. there is a renormalization scheme selection, which is chosen depending on the properties of the parameters needed in the calculation. As in the factorization procedure, the renormalization introduces a scale, $\mu_{R}$, at which the renormalization is performed and any physical observable should be invariant under variations of this scale. However, a theoretical dependence is obtained in $\mathrm{pQCD}$ since the perturbative expansion is performed only to a given order.

\subsubsection{Parton Distribution Functions}

The partonic structure of hadrons plays a fundamental role in elementary particle physics. The comparison of data with SM predictions, precision measurements of SM parameters, and searches for signals of physics beyond the SM, all rely on the parton picture of hadronic beam particles.

As already mentioned, pQCD is not able to predict the $\mathrm{x}$-dependence of the PDFs. PDFs at a given scale $Q_{0}^{2}$ are extracted from fits to data and DGLAP equations are used to predict PDFs to a higher scale $Q^{2}$. The PDFs are parametrized and the parameters are determined by a $\chi^{2}$ minimization over data from different type of measurements: structure functions in deep-inelastic $e, \mu$ or $\nu$ scatter-

ing, measurements of Drell-Yan production, $\mathrm{W}$-asymmetry in $p \bar{p}$ collisions and 
inclusive jet cross sections. Different groups provide parameterizations of parton densities. Among others, PDFs come from Martin, Roberts, Stirling and Thorne (MRST) group [10] and the "Coordinated Theoretical-Experimental Project on QCD" ( CTEQ Collaboration) [11].

\subsubsection{PDFs uncertainties}

A Hessian method is used to evaluate the PDFs uncertainties. A brief description of the method is given below, for more details see [12,13].

In the Hessian method, a large matrix $(20 \times 20$ for CTEQ, $15 \times 15$ for MRST), with dimensions equal to the number of free parameters in the fit, has to be diagonalized. The result is 20 (15) orthogonal eigenvectors for CTEQ (MRST), denoted as $a_{i}$, which provides the basis for the determination of the PDFs uncertainties for any cross section. The Hessian matrix can be expressed as:

$$
H_{i j}=\frac{1}{2} \frac{\partial^{2} \hat{\chi}^{2}}{\partial a_{i} \partial a_{j}}
$$

This matrix determines the behavior of $\hat{\chi}^{2}(a)$ in the neighborhood of the minimum. The point $a_{0}$ in the $n$-dimensional parameter space, where $\hat{\chi}^{2}(a)$ is minimum, is the best fit to the global data set. Points in some small neighborhood of $a_{0}$ are also acceptable fits. For each eigenvector two displacements from $a_{0}$, in the + and - directions along the vector, denoted $a_{i}^{+}$and $a_{i}^{-}$for the $i^{t h}$ eigenvector are considered. At these points, $\hat{\chi}^{2}=\hat{\chi}_{0}^{2}+T^{2}$ where $\hat{\chi}_{0}^{2}=\hat{\chi}^{2}\left(a_{0}\right)=$ the minimum, and $T$ is a parameter called tolerance. Any PDFs set with $\hat{\chi}^{2}-\hat{\chi}_{0}^{2}<T^{2}$ is considered to be an acceptable fit to the global data set. In particular, the $2 n$ PDFs sets $a_{i}^{ \pm}$span the parameter space in the neighborhood of the minimum. CTEQ group chooses $T^{2} \sim 100$ and MRST group uses $T^{2} \sim 50$.

Any quantity $\Gamma$ that depends on PDFs has a predicted value $\Gamma_{0}=\Gamma\left(a_{0}\right)$ and an associated, a priori asymmetric, uncertainty $\delta \Gamma$. The $+(-)$ uncertainties are calculated as:

$$
\delta \Gamma_{+}=\left(\sum_{k=1}^{n}\left[\max \left(\Gamma\left(a_{i}^{+}\right), \Gamma\left(a_{i}^{-}\right), \Gamma\left(a_{0}\right)\right)-\Gamma\left(a_{0}\right)\right]^{2}\right)^{1 / 2}
$$


and

$$
\delta \Gamma_{-}=\left(\sum_{k=1}^{n}\left[\min \left(\Gamma\left(a_{i}^{+}\right), \Gamma\left(a_{i}^{-}\right), \Gamma\left(a_{0}\right)\right)-\Gamma\left(a_{0}\right)\right]^{2}\right)^{1 / 2}
$$

In figure 2.3 the uncertainties on gluon and u-quark distributions are shown. The $\mathrm{u}$-quark distribution is tightly constrained for $\mathrm{x} \leq 0.8$, whereas the uncertainty on the gluon distribution can be larger than a factor of 2 at high $\mathrm{x}$.
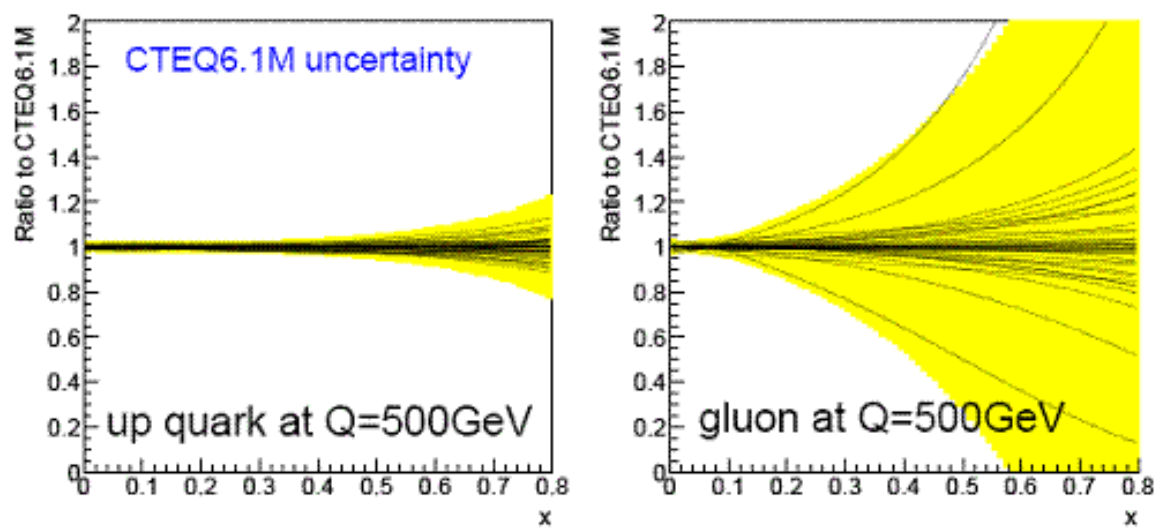

Figure 2.3: Uncertainty on gluon and u-quark PDFs. The yellow bands represent the global uncertainty. The curves are the ratios of the 40 eigenvector basis sets to the standard set, CTEQ6.1M.

\subsubsection{Electroweak Theory}

The weak theory was proposed by Enrico Fermi in 1934 in order to explain the proton $\beta$-decay [14]. In this theory four fermions directly interacted with one another in such a way that a neutron (or a down-quark) could be directly split into an electron, an antineutrino and a proton (an up-quark). The strength of the Fermi's interaction was given by the Fermi constant, $G_{F}$.

Feynman diagrams described the interaction remarkably well at tree level but loop diagrams could not be calculated reliably because Fermi's interaction was not renormalizable. The solution came in 1967 when the electromagnetic and weak 
interactions were successfully unified by Glashow, Salam and Weinberg [15-17]. This unification constituted the Standard Electroweak Model which is the core of the SM. The idea of the unification is to combine both interactions into one single theoretical framework in which they would appear as two manifestations of the same fundamental interaction. These interactions are unified under the group $\mathrm{SU}(2)_{L} \otimes \mathrm{U}(1)_{\mathrm{Y}}$. The first part of the group has dimension three and therefore, three generators are needed: $t_{i}=\frac{\sigma_{i}}{2}(\mathrm{i}=1,2,3)$ where $\sigma_{i}$ are the Pauli matrices. These generators, due to the global gauge invariance under $\mathrm{SU}(2)$, introduce a new quantum number called the weak isospin $(T)$. This number is associated to the different spin-like multiplets. Since weak force only interacts with lefthanded particles (right-handed antiparticles), the left-handed fermions transform as doublets while the right handed ones transform as singlets:

$$
\begin{aligned}
f_{L}^{i} & =\left(\begin{array}{c}
\nu_{L}^{i} \\
l_{L}^{i}
\end{array}\right),\left(\begin{array}{c}
u_{L}^{i} \\
d_{L}^{i}
\end{array}\right) \\
f_{R}^{i} & =l_{R}^{i}, u_{R}^{i}, d_{R}^{i}
\end{aligned}
$$

where $i=1,2,3$ corresponds to the family index. Hence, the weak interaction is divided into a "charged part" (that is, exchanging the components of the doublet) and a "neutral part" (that is, leaving the doublets as they are). Since $\mathrm{SU}(2)$ is a non-Abelian group, it allows self-interactions of these gauge fields.

Since the group $U(1)_{Y}$ has only one dimension, its structure is more simple having only one generator called the hypercharge $\hat{Y}$. Once the $\mathrm{SU}(2)_{L} \otimes \mathrm{U}(1)_{\mathrm{Y}}$ group is defined, the SM electroweak Lagrangian is obtained by requiring invariance under local gauge transformations to obtain an interacting field theory, following the analogy with QED. This is achieved by replacing the derivatives of the fields by the corresponding covariant derivative, which now has the form:

$$
D_{\mu} \equiv \partial_{\mu}-i g \boldsymbol{T} \boldsymbol{W}_{\mu}-i g^{\prime} \frac{Y}{2} B_{\mu}
$$

where g and g' are the coupling constants corresponding to $S U(2)_{L}$ and $U(1)_{Y}$, respectively. 
Then, the electroweak Lagrangian can be written as:

$$
\mathcal{L}_{E W}=\mathcal{L}_{f}+\mathcal{L}_{G}+\mathcal{L}_{S S B}+\mathcal{L}_{Y W}
$$

The first term corresponds to the fermion Lagrangian:

$$
\mathcal{L}_{f}=\sum_{f=l, q} \bar{f} i \not D f
$$

The second term is the contribution from the gauge fields:

$$
\mathcal{L}_{G}=-\frac{1}{4} W_{\mu \nu}^{i} W_{i}^{\mu \nu}-\frac{1}{4} B_{\mu \nu} B^{\mu \nu}+\mathcal{L}_{G F}+\mathcal{L}_{F P}
$$

where $W_{\mu \nu}^{i}$ (with $i=1,2,3$ ) and $B_{\mu \nu}$ are, respectively, the field strength tensors for $S U(2)_{L}$ and $U(1)_{Y}$ defined as:

$$
\begin{aligned}
W_{\mu \nu}^{i} & \equiv \partial_{\mu} W_{\nu}^{i}-\partial_{\nu} W_{\mu}^{i}+g \epsilon^{i j k} W_{\mu}^{j} W_{\nu}^{k} \\
B_{\mu \nu} & \equiv \partial_{\mu} B_{\nu}-\partial_{\nu} B_{\mu}
\end{aligned}
$$

and $\mathcal{L}_{G F}$ and $\mathcal{L}_{F P}$ are the gauge fixing and Faddeev Popov Lagrangians that are needed in any theory [18].

The last two terms of the electroweak Lagrangian equation (2.19) are the symmetry breaking sector and the Yukawa Lagrangian, respectively, which will be described in next subsection.

The gauge fields presented at equation (2.22) can be rewritten as:

$$
\begin{aligned}
W_{\mu}^{ \pm} & =\frac{1}{\sqrt{2}}\left(W_{\mu}^{1} \mp i W_{\mu}^{2}\right) \\
Z_{\mu} & =\cos \theta_{W} W_{\mu}^{3}-\sin \theta_{W} B_{\mu} \\
A_{\mu} & =\sin \theta_{W} W_{\mu}^{3}+\cos \theta_{W} B_{\mu}
\end{aligned}
$$

where, again, $A_{\mu}$ represents the photon field and $\cos \theta_{W}=\frac{g}{\sqrt{g^{\prime 2}+g^{2}}}$ is the weak mixing angle, which relates both couplings by the simple relation $\tan \theta_{W}=g^{\prime} / g$. In addition, $W_{\mu}^{ \pm}$and $Z_{\mu}$ fields are associated to the physical $W^{ \pm}$and $Z^{0}$ boson particles. In this framework, the electron charge and the Fermi constant can be 
written in terms of the couplings through the following relations:

$$
\begin{aligned}
e & =g \sin \theta_{W} \\
G_{F} & =\frac{\sqrt{2}}{8} \frac{g^{2}}{m_{W}^{2}} .
\end{aligned}
$$

The electric charge $\hat{Q}$, the third component of the weak isospin $\hat{T}_{3}$, and the weakhypercharge $\hat{Y}$ are linearly related by the Gell-Mann Nishijima formula:

$$
\hat{Q}=\hat{T}_{3}+\hat{Y} / 2
$$

Hence, the global and local conservation of weak-isospin and hypercharge naturally implies charge conservation, as required by QED, and the electromagnetic and weak interactions are unified under the same theoretical framework.

\subsubsection{The Higgs mechanism}

As shown, the Standard Model formalism allows the unification of electromagnetic and weak interactions through the exploitation of a local gauge symmetry. Nevertheless, this gauge symmetry requires massless $W^{ \pm}$and $Z$ bosons. This requirement is in contradiction with the observation and one needs to introduce a mechanism for generating non-zero masses while preserving the renormalizability of the theory. In the SM, the Higgs mechanism of Spontaneous Symmetry Breaking (SSB) is proposed.

In the SSB, one introduce a new field, the Higgs field, such as:

$$
\Phi \equiv\left(\begin{array}{c}
\phi^{+} \\
\phi^{0}
\end{array}\right)
$$

The correspondent kinetic and potential term in the Lagrangian has the form:

$$
\mathcal{L}_{\Phi}=\left(D_{\mu} \Phi\right)^{\dagger} D^{\mu} \Phi-V(\Phi)
$$


where

$$
V(\Phi)=\mu^{2} \Phi^{\dagger} \Phi+\lambda\left(\Phi^{\dagger} \Phi\right)^{2}
$$

If $\lambda>0$ and $\mu^{2}<0$ the potential $V(\Phi)$ has a minimum for:

$$
\Phi^{\dagger} \Phi=-\frac{\mu^{2}}{2 \lambda} \equiv \frac{v^{2}}{2} .
$$

Thus, the field $\Phi$ has a non-zero vacuum expectation value (VEV):

$$
\langle 0|\Phi| 0\rangle=\frac{v}{\sqrt{2}} \neq 0 \text {. }
$$

Choosing one of a set of degenerate states of minimum energy breaks the gauge symmetry.

As stated by the Goldstone theorem, fields that acquire a VEV will have an associated massless Goldstone boson which will disappear transformed into the longitudinal component of a massive gauge boson. Since the photon is known to be massless, the symmetry is chosen to be broken so that only the fields with zero electric charge (the ones that cannot couple to the electromagnetic interaction) acquire a VEV. In such a way, the symmetry of the photon-associated operator, $\hat{Q}$ is preserved:

$$
\begin{gathered}
\Phi_{0} \equiv\langle 0|\Phi| 0\rangle \equiv\left(\begin{array}{l}
0 \\
v
\end{array}\right) \\
Q \Phi_{0}=0 .
\end{gathered}
$$

Expanding around the true minimum of the theory, the complex field $\phi$ becomes:

$$
\Phi(x)=e^{i \frac{\boldsymbol{\tau}}{2} \boldsymbol{\xi}(x)} \frac{1}{\sqrt{2}}\left(\begin{array}{c}
0 \\
v+H(x)
\end{array}\right) .
$$

where the three parameters $\boldsymbol{\xi}(x)$ correspond to the motion through the degenerated minima in the $\mathrm{SU}(2)$ space. Since the Lagrangian is locally gauge invariant, one 


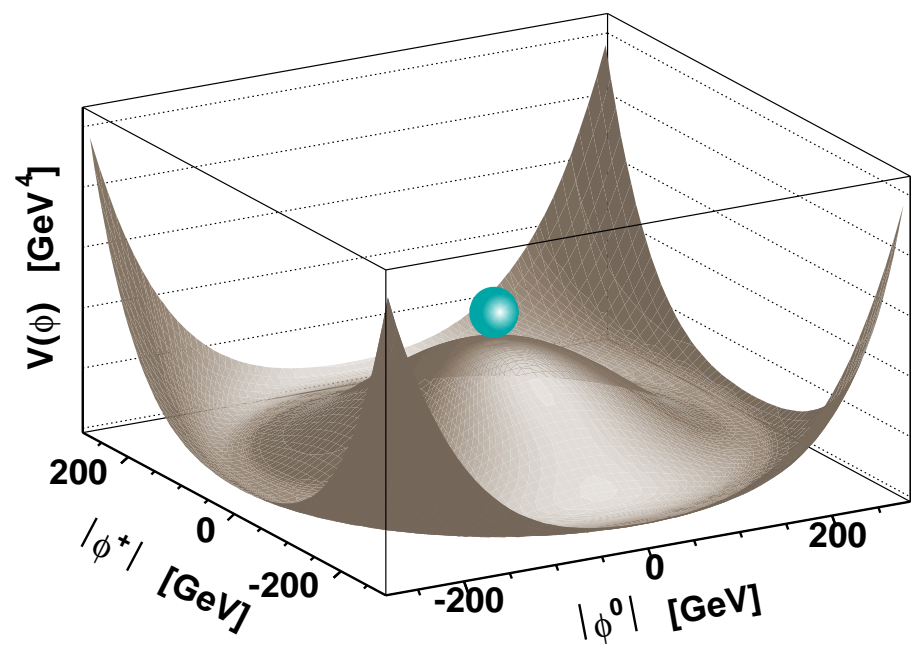

Figure 2.4: The minimum of the Higgs potential occurs at $-\mu^{2} /(2 \lambda)$, not at zero

can choose $\boldsymbol{\xi}(x)=0$. Hence, introducing this expansion into the SM Lagrangian equation (2.19), one obtains tree level predictions for massive fermions (coming from the $\mathcal{L}_{Y W}$ part), massive gauge bosons (coming from the kinetic part of $\mathcal{L}_{S S B}$ and a new Higgs boson. These relations are:

$$
\begin{aligned}
M_{W} & =\frac{v g}{2} \\
M_{Z} & =v \frac{\sqrt{g^{2}+g^{\prime 2}}}{2} \\
M_{H} & =\sqrt{-2 \mu^{2}}=\sqrt{2 \lambda} v \\
m_{f} & =\lambda_{f} \frac{v}{\sqrt{2}} \\
m_{\gamma}^{2} & =0
\end{aligned}
$$

where $f$ stands for the fermions in the theory. These relations can also be expressed as a function of the weak mixing angle,

$$
M_{z}=\frac{\frac{1}{2} v g}{\cos \theta_{W}},
$$


which leads to the SM prediction

$$
\frac{M_{W}^{2}}{M_{Z}^{2}}=\cos ^{2} \theta_{W} .
$$

This prediction was tested once the $W^{ \pm}$and $Z$ vector bosons where discovered in 1983 by UA1 and UA2 collaborations at the CERN SPS $[19,20]$.

The ten independent fields before SSB (three massless gauge bosons $\left(W^{ \pm}, Z\right)$, with two polarization states each, and one $\mathrm{SU}(2)$ doublet of complex scalars) are now represented by three massive bosons, which account for nine degrees of freedom, and a new physical scalar particle called the Higgs boson, which accounts for the last degree of freedom.

This new particle, which is the missing piece to confirm the Higgs mechanism, has the couplings completely defined by the theory:

$$
\begin{aligned}
\lambda_{H H H} & =3 \frac{M_{H}^{2}}{M_{Z}^{2}} \\
\lambda_{H V V} & =2 \sqrt{2 G_{F}} M_{V}^{2} \\
\lambda_{H f f} & =2 \sqrt{2 G_{F}} m_{f}
\end{aligned}
$$

where $V=W, Z$ and $G_{F}$ is the Fermi constant. The vacuum expectation value $v$ is determined experimentally from the partial width $\Gamma\left(\mu \rightarrow \nu_{\mu} \bar{\nu}_{e} e\right)$ at low energies $\left(q^{2}<<M_{W}^{2}\right)$ :

$$
\frac{G_{F}}{\sqrt{2}}=\frac{g^{2}}{8 M_{W}^{2}}=\frac{1}{2 v^{2}},
$$

where, substituting experimental values:

$$
v=\left(\sqrt{2} G_{F}\right)^{-\frac{1}{2}}=246 \mathrm{GeV},
$$

which sets the electroweak symmetry breaking scale.

This new particle allows Yukawa-like terms in the Lagrangian:

$$
g_{f}\left[\left(\bar{f}_{L} \phi\right) f_{R}+\text { h.c. }\right] \text {, }
$$


which can be written in terms of the VEV:

$$
\sqrt{\frac{1}{2}} g_{f} v\left(\bar{f}_{L} f_{R}+\bar{f}_{R} f_{L}\right)
$$

Therefore, not only the bosons acquire mass through the Higgs mechanism but also the fermions with $m_{f}=g_{f} v / \sqrt{2}$. Noticeably, the strength of the coupling is proportional to the masses. However, masses are not predicted unless $g_{f}$ is determined.

\subsubsection{Standard Model Limitations}

The SM description of the different processes involving electroweak or strong interactions is extremely accurate. At the present time, no experiment has been able to find any clear deviation from the SM predictions. Nevertheless, physicists are still pushing to find such tiny deviations. The main reason is that the SM has serious theoretically motivated problems, starting from the fact that gravity is not accommodated in the theory, that prevent it from being the ultimate theory, the Theory of Everything (TOE), that would describe nature in a comprehensive manner.

Even accepting the peculiar set of group representations and hypercharges required by the model, the SM contains at least 19 free parameters, such as couplings, masses and mixings, which cannot be predicted but must be measured by the experiment. In addition, more parameters would be needed if one wants to accommodate non-accelerator observations such as the cosmological baryon asymmetry, neutrino masses and mixings or the problematic cosmological constant.

The SM lets also several questions unanswered such as why are there three generations, spatial dimensions or colors, how do we understand neutrino oscillations and massive neutrinos, why are the electric charge of the proton and the electron exactly opposite or whether the Higgs mechanism is really the process through which the electroweak symmetry breaking occurs and lay beneath the origin of masses. In addition, the model cannot explain which are the mechanisms to produce the matter-antimatter asymmetry observed in the universe or what is the relation between the strong and electroweak forces. 
Perhaps the most surprising feature of the SM is the accurate description of the interactions between particles with masses 17 orders of magnitude smaller than the Planck mass and the difficulty to accommodate gravity within this framework [21]. This feature may be an indication that the SM is an effective theory, that is a "low energy" limit of a more fundamental one. But this assumption automatically leads to the question of up to which energy scale will the SM be valid.

However, spin zero fields are radically different from fermions and gauge bosons. The latter are protected from large radiative corrections to their masses thanks to chiral and gauge symmetries, respectively. In the SM there is no mechanism to prevent scalar particles from acquiring large masses through radiative corrections. Therefore, $m_{H}^{2}$ receives enormous quantum corrections from the virtual effects of every particle which couples to the Higgs field.

Due to these corrections, the Higgs mass would be

$$
m_{h_{S M}}^{2}=\left(m_{h}^{2}\right)_{0}+\Delta M_{H}^{2}
$$

where $\left(m_{h}^{2}\right)_{0}$ is the bare Higgs mass and $\Delta M_{H}^{2}$ is the correction given by

$$
\Delta M_{H}^{2}=-\frac{\lambda_{f}^{2}}{16 \pi^{2}}\left[2 \Lambda^{2}+\mathcal{O}\left(m_{f}^{2} \ln \left(\frac{\Lambda}{m_{f}}\right)\right)\right] i
$$

where $\lambda_{f}$ is the Yukawa coupling of the fermion $f$ and $\Lambda$ is an energy cutoff which is interpreted as the energy scale at which new physics enters and changes the high-energy behavior of the theory. If the SM needs to describe nature until the Planck scale, then the quantum correction $\Delta M_{H}^{2}$ is about 30 orders of magnitude larger than the bare Higgs mass square. A cancellation of these corrections at all orders would call for an incredible "fine tinning" which seems very unlikely [22]. This problem is present even if there is no direct coupling between the Standard model Higgs boson and the unknown heavy particles [23].

In a model with spontaneous electroweak symmetry breaking, the problem affects not only to the Higgs mass but also its expectation value and the masses of other particles that get their masses through this mechanism such as the $W, Z$, quarks and charged leptons. This situation has also an analogy with the self-energy corrections on the electron, which is solved by the presence of the positron [24]. 
Hence, it is unnatural to have all the SM particles masses at the electroweak scale unless the model is somehow cut off and embedded in a richer structure at energies no bigger than the $\mathrm{TeV}$ scale.

\subsection{Minimal Supersymmetric Extension of the Stan- dard Model}

After a brief introduction to supersymmetry, this section presents the Minimal Supersymmetric Extension of the Standard Model (MSSM).

\subsubsection{Supersymmetry}

Supersymmetry (SUSY) [4][23] is a symmetry which relates masses and couplings of bosons and fermions via spin- $\frac{1}{2}$ charges. In SUSY, particles are combined into superfields and an operator $Q$ generates the transformation of converting fermions to bosons and vice versa:

$$
\left.\left.Q \mid \text { Boson }\rangle=\mid \text { Fermion }\rangle \quad Q^{\dagger} \mid \text { Fermion }\right\rangle=\mid \text { Boson }\right\rangle
$$

Therefore $Q$ is a complex anticommuting spinor and its hermitian conjugate, $Q^{\dagger}$, is also a symmetry generator. Both generators are fermionic in nature $(S=1 / 2)$ and form a Lie algebra [25], together with the four-momentum and the Lorentz transformation generators. In fact, SUSY is a generalization of the space-time symmetries of quantum field theory and seems to be the last possible extension of the Lorentz group [26].

In this situation, each chiral fermion $f_{L, R}$ has a scalar partner $\tilde{f}_{L, R}$ and for each massless gauge boson $A_{\mu}$, with the helicity states \pm 1 , there is a massless spin $1 / 2$ gaugino partner, with helicity states $\pm \frac{1}{2}$. 


\subsubsection{Supersymmetry and the hierarchy problem}

The SM hierarchy problem presented in section 2.1 .5 is very elegantly solved when considering the supersymmetric theory [27]. The reason is that every fermion $f$ has a scalar SUSY partner $S$ that couples to the Higgs as well and contributes with a mass correction term of the form:

$$
\Delta M_{H}^{2}=\frac{\lambda_{S}^{2}}{16 \pi^{2}}\left[2 \Lambda^{2}+\mathcal{O}\left(m_{S}^{2} \ln \left(\frac{\Lambda}{m_{S}}\right)\right)\right]
$$

Since now $\lambda_{f}=\lambda_{S}$ and Fermi statistics imply an opposite sign with respect to the contribution stated in equation (2.52), all the terms have a counter-term that naturally cancel all the huge corrections. The terms that do not cancel are of the form:

$$
\Delta M_{H}^{2}=\frac{\lambda^{2}}{16 \pi^{2}}\left|m_{S}^{2}-m_{f}^{2}\right|
$$

where some smaller contributions have been omitted. This result leads us to the following "naturalness" argument, [28,29]: since these corrections must not be greater than $m_{h_{S M}}$ in order to avoid too much fine tuning, then

$$
\left|m_{S}^{2}-m_{f}^{2}\right| \lesssim 1 \mathrm{TeV}^{2}
$$

Hence, one associates $\Lambda \sim 1 \mathrm{TeV}$ as the scale where the $\mathrm{SM}$ is no longer valid and must be substituted by its supersymmetric extension. As a benefit, this new theory would be valid all the way up to the Planck scale. In any case, this is only a qualitative argument and does not help predicting exactly whether new particles should appear at $900 \mathrm{GeV}$ or $2 \mathrm{TeV}$.

\subsubsection{Other benefits from the introduction of SUSY}

Besides making a small Higgs mass natural, SUSY has other interesting consequences. One of them is that when SUSY is locally realized it contains among its gauge fields the graviton. Thus SUSY seems to be a good candidate for a theory

of all interactions, or at least to play an important role in any such theory. In 
addition, Great Unifications Theories (GUT) also provide good motivation for the existence of supersymmetry. One can use the running of the three couplings of the SM, measured at the electroweak scale, and find that, at a certain GUT scale of $10^{15} \mathrm{GeV}$, the couplings almost become the same value [30]. But if one considers SUSY then the couplings are modified in such a way that they become precisely the same value at the GUT scale. Therefore, it is a strong indication for the need of SUSY. However, some people claim that there is nothing special on that [31] provided that other models could do it if they introduce as many parameters as SUSY does.

In addition to gauge coupling unification, SUSY is also a key ingredient for GUT. These theories have interesting predictions such as a small neutrino mass of the order of $m_{\nu} \approx m_{W}^{2} / m_{G U T} \approx 10^{-2} \mathrm{eV} / \mathrm{c}^{2}$ and it can lead to the understanding of the different quark and lepton quantum numbers. But without SUSY the lifetime of the proton would be too small and the prediction for $\sin ^{2} \theta_{W}$ would differ from the experiment [31-33]. In addition, SUSY has been of greatest interest in string theories since it is the mechanism which provides a coherent and complete framework which avoids negative square masses in some vibrational modes (tachyons) [34].

Furthermore, some SUSY models predict the presence of a lightest supersymmetric particle, which is a candidate for dark matter in the universe, provided that it is neutral, weakly interacting and absolutely stable.

As a final remark, recent fits on the electroweak precision observables, such as the effective leptonic weak mixing angle, $\sin ^{2} \theta_{\text {eff }}$, seem to favor supersymmetric models in front of the SM alone [35]. This can be seen in, where the SM predictions for the $M_{W}$ as a function of $m_{t}$ is being compared with the predictions from the unconstrained Minimal Supersymmetric Standard Model (MSSM), which will be described in the next subsection. The predictions within the two models give rise to two bands with only a relatively small overlap region. The allowed parameter region in the SM arises from varying the only free parameter of the model, the mass of the SM Higgs boson from $M_{h_{S M}}=114 \mathrm{GeV} / c^{2}$ (upper edge of the band) to $400 \mathrm{GeV} / c^{2}$ (lower edge of the band). For the MSSM area, SUSY masses close to their experimental limit are assumed for the upper edge, while the MSSM with large masses yields the lower edge of the blue area (dark-shaded). The $68 \%$ C.L. 
experimental results slightly favours the MSSM over the $\mathrm{SM}^{2}$.

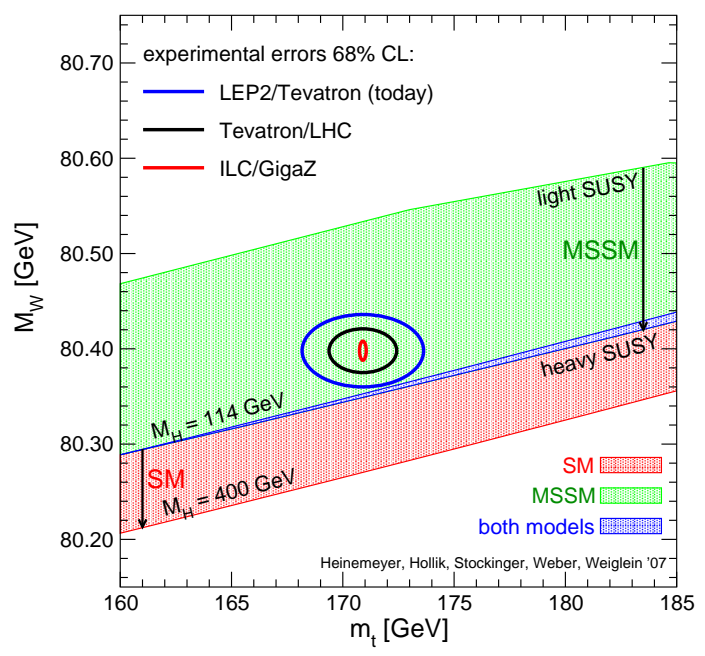

Figure 2.5: $M_{W}$ as a function of $m_{t}$ as predicted by the SM in red (mediumshaded) and blue (dark-shaded) bands and with the MSSM prediction in green (lightshaded) and blue (dark-shaded) bands. The perspectives for the present and future generation colliders, are also stated.

\subsubsection{The Minimal Supersymmetric Standard Model}

Similarly to the SM construction, that was conceived to be the minimal group viable to explain the electroweak sector, the MSSM [36] is the minimal viable supersymmetric extension of the SM. The MSSM obeys the same $\mathrm{SU}(3)_{C} \otimes \mathrm{SU}(2)_{L} \otimes$ $\mathrm{U}(1)_{\mathrm{Y}}$ gauge symmetries of the Standard Model but doubles the spectrum of new particles since for every particle in the SM, a superpartner is postulated which differs by half a unit of spin. The superpartners are conveniently described by a notation with close correspondence to the SM notation for bosons and fermions. Hence, the superpartners are written with the same letter of their partner but with a tilde over it and the superfields are written with a "hat" superscript. In addition, the bosonic partners of the fermions are denoted starting with an extra "s" (e.g. selectron is the superpartner of the electron) and the fermionic partners

\footnotetext{
${ }^{2}$ Last top mass measurements from the Tevatron [5] indicate even a lower mass for the top: $m_{t}=171.4 \pm 1.2$ (stat) \pm 1.8 (syst) $\mathrm{GeV} / c^{2}$.
} 
of the bosons finish with the suffix "ino" (e.g. gluino is the superpartner of the gluon).

For simplicity and to avoid unnecessary repetitions, consider the case of one generation of quarks, leptons and their superpartners. One can define $\hat{Q}$ as the superfield containing an $\mathrm{SU}(2)_{L}$ doublet of quarks:

$$
Q=\left(\begin{array}{l}
u_{L} \\
d_{L}
\end{array}\right)
$$

and their scalar partners which are also in an $\mathrm{SU}(2)_{L}$ doublet,

$$
\tilde{Q}=\left(\begin{array}{c}
\tilde{u}_{L} \\
\tilde{d}_{L}
\end{array}\right)
$$

In an analogous form, the superfield $\hat{U}^{c}\left(\hat{D}^{c}\right)$ contains the right-handed up (down) anti-quark, $\bar{u}_{R}\left(\bar{d}_{R}\right)$, and its scalar partner, $\tilde{u}_{R}^{*}\left(\tilde{d}_{R}^{*}\right)$. Following the same pattern, leptons are contained in the $S U(2)_{L}$ doublet superfield $\hat{L}$ which contains the lefthanded fermions,

$$
L=\left(\begin{array}{c}
\nu_{L} \\
e_{L}
\end{array}\right)
$$

and their scalar partners,

$$
\tilde{L}=\left(\begin{array}{c}
\tilde{\nu}_{L} \\
\tilde{e}_{L}
\end{array}\right) \quad \text {. }
$$

Finally, the superfield $\hat{E}^{c}$ contains the right-handed anti-electron, $\bar{e}_{R}$, and its scalar partner, $\tilde{e}_{R}^{*}$.

Similarly, for every gauge boson it exist a Majorana fermion (gaugino). $\hat{G}^{a}$ is defined as a superfield that contains all the gluons, $g^{a}$, and their fermion partners the gluinos, $\tilde{g}^{a} ; \hat{W}_{i}$ contains the $S U(2)_{L}$ gauge bosons, $W_{i}$, and their fermion partners, $\tilde{\omega}_{i}$ (winos); and $\tilde{B}$ contains the $U(1)$ gauge field, $B$, and its fermion 
partner, $\tilde{b}$ (bino).

In addition, in the MSSM the Higgs sector is enlarged to avoid triangle gauge anomalies [37-39]. Gauge theories cannot have anomalies and this is simply achieved by requiring that the sum of all fermion charges vanishes. The Higgs scalar doublet acquires a SUSY partner which is an $\mathrm{SU}(2)_{L}$ doublet of Majorana fermion fields, $\tilde{h}_{1}$ (Higgsinos), which will contribute to the triangle $\mathrm{SU}(2)_{L}$ and $\mathrm{U}(1)_{Y}$ gauge anomalies. Since fermions in SM have exactly the right quantum numbers to cancel these anomalies, it follows that the contribution from the fermionic partner of the Higgs doublet remains uncanceled. The easiest solution is to require a second Higgs doublet with precisely the opposite $\mathrm{U}(1)_{Y}$ quantum number than the first Higgs doublet. Furthermore, in the SM the Higgs doublet (the complex conjugate of the doublet) can couple to the $T_{3}=+\frac{1}{2}\left(T_{3}=-\frac{1}{2}\right)$ fermions and give mass to all the spectrum of fermions. But, in a supersymmetric theory, any doublet can give mass either to a $T_{3}=+\frac{1}{2}$ or a $T_{3}=-\frac{1}{2}$ fermion but not both. Thus, two Higgs doublets are needed in order to generate both up-like and down-like quark masses. As result, one could think of the SM becoming a two Higgs doublet model (2HDM) [40] prior to introduce the supersymmetric sector. In Table 2.3 the spectrum of the MSSM fields is summarized.

With two SU(2) doublets, the theory has eight real scalar fields and three massless gauge bosons, which accounts for fourteen degrees of freedom. After SUSY breaking, the three gauge bosons acquire masses (nine degrees of freedom), which means that there should exist five spin-zero Higgs fields in the spectrum: three neutral scalars $(h, H, A)$ and two charged pairs $\left(H^{+}, H^{-}\right)$.

The parameters of the supersymmetry-conserving sector consist of:

- Gauge couplings: $g_{s}, g$ and $g^{\prime}$, corresponding to the Standard Model gauge group $\mathrm{SU}(3)_{C} \otimes \mathrm{SU}(2)_{L} \otimes \mathrm{U}(1)_{\mathrm{Y}}$, respectively.

- Higgs mass parameter, $\mu$.

- Higgs-fermion Yukawa coupling constants: $\lambda_{u}, \lambda_{d}$, and $\lambda_{e}$, corresponding to the coupling of quarks or leptons and their superpartners to the Higgs bosons and higgsinos. 


\begin{tabular}{cc|cc|cc|c}
\hline \hline \multirow{2}{*}{ Names } & & 2HDM particle & SUSY partner & $\left(\begin{array}{c}\mathbf{S U}(\mathbf{3})_{\boldsymbol{C}} \\
\mathbf{S U}(\mathbf{2})_{\mathbf{L}} \\
\mathbf{U}(\mathbf{1})_{\boldsymbol{Y}}\end{array}\right)$ \\
\hline \multirow{2}{*}{ squarks, quarks } & $\hat{Q}$ & $\left(u_{L} d_{L}\right)$ & $\frac{1}{2}$ & $\left(\tilde{u}_{L} \tilde{d}_{L}\right)$ & 0 & $\left(3,2, \frac{1}{3}\right)$ \\
$(\times 3$ families $)$ & $\hat{U}$ & $u_{R}^{\dagger}$ & $\frac{1}{2}$ & $\tilde{u}_{R}^{*}$ & 0 & $\left(\overline{3}, 1,-\frac{4}{3}\right)$ \\
& $\hat{D}$ & $d_{R}^{\dagger}$ & $\frac{1}{2}$ & $\tilde{d}_{R}^{*}$ & 0 & $\left(\overline{3}, 1, \frac{2}{3}\right)$ \\
\cline { 2 - 7 } sleptons, leptons & $\hat{L}$ & $\left(\nu e_{L}\right)$ & $\frac{1}{2}$ & $\left(\tilde{\nu} \tilde{e}_{L}\right)$ & 0 & $(1,2,-1)$ \\
$(\times 3$ families $)$ & $\hat{E}$ & $e_{R}^{\dagger}$ & $\frac{1}{2}$ & $\tilde{e}_{R}^{*}$ & 0 & $(1,1,2)$ \\
\hline \multirow{2}{*}{ EWK bosons } & $\hat{W}$ & $W^{1} W^{2} W^{3}$ & 1 & $\tilde{W}^{1} \tilde{W}^{2} \tilde{W}^{3}$ & $\frac{1}{2}$ & $(1,3,0)$ \\
Strong bosons & $\hat{B}$ & $B$ & 1 & $\tilde{B}_{a}$ & $\frac{1}{2}$ & $(1,1,0)$ \\
\cline { 2 - 7 } Higgs, higgsinos & $\hat{G}_{a}$ & $g_{a}$ & 1 & $\tilde{g}_{a}$ & $\frac{1}{2}$ & $(8,1,0)$ \\
& $\hat{H}_{d}$ & $\left(H_{u}^{+} H_{u}^{0}\right)$ & 0 & $\left(\tilde{H}_{u}^{+} \tilde{H}_{u}^{0}\right)$ & $\frac{1}{2}$ & $(1,2,1)$ \\
\hline \hline
\end{tabular}

Table 2.3: Superfields and particle content of the MSSM. Symbols for each of the chiral supermultiplets as a whole are indicated in the second column.

The supersymmetry-breaking sector contains the following set of parameters:

- Gaugino Majorana masses $M_{3}, M_{2}$ and $M_{1}$, associated with the $\mathrm{SU}(3)_{C}, \mathrm{SU}(2)_{L}$ and $\mathrm{U}(1)_{\mathrm{Y}}$ subgroups, respectively. These masses may be connected in some cases as will be seen later.

- Five scalar squared-mass parameters for the squarks and sleptons: $M_{\tilde{Q}}^{2}, M_{\tilde{U}}^{2}$, $M_{\tilde{D}}^{2}, M_{\tilde{L}}^{2}$ and $M_{\tilde{E}}^{2}$, corresponding to the five electroweak gauge multiplets.

- Trilinear interaction terms of the form Higgs-squark-squark and Higgs-sleptonslepton, with coefficients $A_{u}, A_{d}$ and $A_{e}$.

- Three scalar Higgs squared-mass parameters, two of which $\left(m_{1}^{2}\right.$ and $\left.m_{2}^{2}\right)$ contribute to the diagonal Higgs squared-masses and a third which corresponds to the off-diagonal terms $m_{12}^{2} \equiv \mu B$. These three parameters can be reexpressed in terms of the two Higgs vacuum expectation values $\left(v_{d}=\left\langle H_{d}^{0}\right\rangle\right.$ 
and $\left.v_{u}=\left\langle H_{u}^{0}\right\rangle\right)^{3}$, usually taken through the ratio

$$
\tan \beta \equiv \frac{v_{u}}{v_{d}}
$$

and one physical Higgs mass ${ }^{4}$.

The gluino is the color octet Majorana (there is no distinct antigluon) fermion partner of the gluon. It has 16 degrees of freedom since there are 8 massless gluons ( 2 spin degrees of freedom, each). The supersymmetric partners of the electroweak gauge and Higgs bosons (gauginos and higgsinos) can mix. As a result, the physical mass eigenstates are model-dependent linear combinations of these states, called charginos and neutralinos, which are obtained by diagonalizing the corresponding mass matrices. There are two charginos $\left(\tilde{\chi}_{i}^{ \pm}\right)$and four neutralinos $\left(\tilde{\chi}_{i}^{0}\right)$, which are by convention ordered in masses $\left(\tilde{\chi}_{1}^{ \pm}\right.$is the lowest chargino and $\tilde{\chi}_{1}^{0}$ is the lowest neutralino). Depending whether the chargino or neutralino eigenstate approximates a particular gaugino or higgsino state, they can become more photino-like, bino-like... and result in strikingly different phenomenology.

The supersymmetric partners of the quarks and leptons are spin-zero bosons and the resulting squarks and sleptons can also mix their left- and right-handed components yielding the mass eigenstates (denoted by the indices 1,2 instead of $L, R$ ). This mixing is proportional to the mass of the SM partner quark or lepton and to $\tan \beta$. Thus, the mixing can lead to an important splitting in the mass spectrum of heavy squarks, specially at large $\tan \beta$. In contrast, the first two families can be considered degenerate in mass. All physical particles of the MSSM are given in Table 2.4.

\subsubsection{MSSM Lagrangian and R-parity}

The MSSM Lagrangian is constructed using the already defined particle content and following an analogy with the $\mathcal{L}_{\mathrm{SM}}$. Following a similar notation as in the SM,

\footnotetext{
${ }^{3}$ Notation $v_{u}\left(v_{d}\right)$ is used to distinguish vacuum expectation values of the Higgs field which couples exclusively to up-type (down-type) quarks.

${ }^{4}$ Note that $v_{d}^{2}+v_{u}^{2}=4 M_{W}^{2} / g^{2}=\left(246 \mathrm{GeV} / c^{2}\right)^{2}$ is fixed by the $W$ mass and the gauge coupling, but $\tan \beta$ is a free parameter of the model.
} 


\begin{tabular}{lcc|llr}
\hline \hline 2HDM & particle & spin & SUSY particle & spin \\
\hline quarks: & $q$ & $\frac{1}{2}$ & squarks: & $\tilde{q}_{1}, \tilde{q}_{2}$ & 0 \\
leptons: & $l$ & $\frac{1}{2}$ & sleptons: & $\tilde{l}_{1}, \tilde{l}_{2}$ & 0 \\
gluons: & $g_{a}$ & 1 & gluinos: & $\tilde{g}_{a}$ & $\frac{1}{2}$ \\
gauge bosons: & $W^{ \pm}, Z^{0}, \gamma$ & 1 & neutralinos: & $\tilde{\chi_{1}^{0}}, \tilde{\chi_{2}^{0}}, \tilde{\chi_{3}^{0}}, \tilde{\chi_{4}^{0}}$ & $\frac{1}{2}$ \\
Higgs bosons: & $h^{0}, H^{0}, A^{0}, H^{ \pm}$ & 0 & charginos: & $\tilde{\chi}_{1}^{ \pm}, \tilde{\chi}_{2}^{ \pm}$ & $\frac{1}{2}$ \\
\hline \hline
\end{tabular}

Table 2.4: The particle content of the MSSM.

the kinetic term of the Lagrangian can be written as:

$$
\begin{aligned}
\mathcal{L}_{K E} & =\sum_{i}\left\{\left(D_{\mu} S_{i}\right)^{\dagger}\left(D^{\mu} S_{i}\right)+\frac{i}{2} \bar{\psi}_{i} \gamma^{\mu} D_{\mu} \psi_{i}\right\} \\
& +\quad \sum_{A}\left\{-\frac{1}{4} F_{\mu \nu}^{A} F^{\mu \nu A}+\frac{i}{2} \bar{\lambda}_{A} D \lambda_{A}\right\} .
\end{aligned}
$$

Here, $S_{i}\left(\psi_{i}\right)$ is the scalar (fermion) component of the $i^{\text {th }}$ chiral superfield, $D$ is the $S U(3) \times S U(2)_{L} \times U(1)$ gauge invariant derivative, $F_{\mu \nu}^{A}$ is the Yang-Mills gauge field and $\lambda_{A}$ is the gaugino superpartner of the corresponding gauge boson. It is worth noticing that the $\sum_{i}$ is a sum over all fermion fields of the SM, the scalar partners and the 2 Higgs doublets with their fermion partners. On the other hand, $\sum_{A}$ is over the $S U(3)_{c}, S U(2)_{L}$ and $U(1)_{Y}$ gauge fields with their fermion partners, the gauginos.

The interactions between bosons and fermions are completely determined by the gauge symmetries and by the supersymmetry:

$$
\begin{aligned}
\mathcal{L}_{i n t}= & -\sqrt{2} \sum_{i, A} g_{A}\left[S_{i}^{*} T^{A} \bar{\psi}_{i L} \lambda_{A}+\text { h.c. }\right] \\
& -\frac{1}{2} \sum_{A}\left(\sum_{i} g_{A} S_{i}^{*} T^{A} S_{i}\right)^{2}
\end{aligned}
$$

where $\psi_{L} \equiv \frac{1}{2}\left(1-\gamma_{5}\right) \psi, T^{A}$ is the matrix of the group generators and $g_{A}$ the gauge coupling constants. It can be seen that there are no adjustable parameter, hence, all interaction strengths are completely fixed in terms of SM coupling 
constants.

Once the superfields and the gauge symmetries are chosen, the only freedom in constructing $\mathcal{L}_{M S S M}$ is contained in a function called superpotential, $\mathcal{W}$. This is an analytic form of the chiral superfields, $\hat{S}$, that has the form:

$$
\mathcal{W}=\epsilon_{i j} \mu \hat{H}_{u}^{i} \hat{H}_{d}^{j}+\epsilon_{i j}\left[\lambda_{L} \hat{H}_{d}^{i} \overline{\hat{L}}^{j} \overline{\hat{E}}+\lambda_{D} \hat{H}_{d}^{i} \hat{Q} \hat{\bar{D}}+\lambda_{U} \hat{H}_{u}^{j} \hat{Q}^{i} \hat{\hat{U}}\right]+W_{R P}
$$

where $i$ and $j$ are $S U(2)_{L}$ doublet indices and $\epsilon_{i j}=-\epsilon j i$ (with $\epsilon_{12}=1$ ) contracts the $S U(2)_{L}$ doublet fields. No derivative interactions are allowed in order that $\mathcal{W}$ be an analytical function. The term $\mu \hat{H}_{u}^{i} \hat{H}_{d}^{j}$ gives mass terms for the Higgs bosons and so $\mu$ is often called the Higgs mass parameter. The terms in the square brackets proportional to $\lambda_{L}, \lambda_{D}$ and $\lambda_{U}$ give the usual Yukawa interactions of the fermions with the Higgs bosons. Hence, unlike the SM case, these coefficients are determined in terms of the fermion masses and the vacuum expectation values of the neutral members of the scalar components, and are not arbitrary couplings.

In the most general superpotential one can add more terms which are grouped under $W_{R P}$ in equation (2.62). These terms are of the form:

$$
W_{R P}=\lambda_{\alpha \beta \gamma} \hat{L}^{\alpha} \hat{L}^{\beta} \overline{\hat{E}}^{\gamma}+\lambda_{\alpha \beta \gamma}^{\prime} \hat{L}^{\alpha} \hat{Q}^{\beta} \overline{\hat{D}}^{\gamma}+\lambda_{\alpha \beta \gamma}^{\prime \prime} \overline{\hat{U}}^{\alpha} \overline{\hat{D}}^{\beta} \overline{\hat{D}}^{\gamma}+\mu^{\prime} \hat{L} \hat{H}
$$

where the indices $\alpha, \beta$ and $\gamma$ label the 3 generations of quarks and leptons. These terms constitute a problem in the sense that the first two contribute to lepton number violation interactions and the third one to baryon number violation interactions $^{5}$. The combination of lepton and baryon violation terms can contribute to the proton decay at tree level through the exchange of the scalar partner of the down quark. Since this process is experimentally restricted [42-45][46-49] it put into question the validity of the model. One solution is to assume that the parameters are small enough to avoid experimental limits. Even this is certainly allowed experimentally, this would imply the introduction of an artificial tuning. The other solution is to introduce a new symmetry called R-parity [50-56]. R-parity $\left(R_{p}\right)$ is

\footnotetext{
${ }^{5}$ The fourth term can be ignored since one can implement a rotation in the lepton field $\hat{L}$ such that this term vanishes [41].
} 
a multiplicative quantum number defined as:

$$
R=(-1)^{3(B-L)+2 s},
$$

where $B$ and $L$ are the baryon and lepton quantum numbers and $s$ is the spin of the particle. Thus, all SM particles have $R_{p}=+1$ while their SUSY partners have $R_{p}=-1$.

The assumption of such a symmetry prevents lepton and baryon number violating terms but has also dramatic phenomenological consequences: there can be no mixing between the sparticles and the $R_{P}=1$ particles, SUSY particles can only be pair-produced in the collisions of SM particles and a SUSY particle would undergo a chain of decays until the lightest SUSY particle (LSP) is produced. Then, this LSP cannot decay further and constitutes a cold dark matter candidate ${ }^{6}$.

\subsubsection{The Minimal Supersymmetric Standard Model Higgs Sec- tor}

As stated in Section 2.2.4, the MSSM Higgs sector extends the SM Higgs sector with a second Higgs doublets. These two doubles generate eight scalar degrees of freedom. Three of them become the longitudinal components of the electroweak bosons, $\mathrm{W}^{ \pm}$and $\mathrm{Z}^{0}$, from the three Goldstone bosons generated with spontaneous symmetry breaking. The remaining five degrees of freedom should manifest as physical states, three neutral and two charged Higgs bosons: the pseudoscalar $\mathrm{A}^{0}$, the $\mathcal{C P}$-even $\mathrm{h}^{0}$ and $\mathrm{H}^{0}$, and the charge $\mathrm{H}^{ \pm}$.

Similarly to the spontaneous symmetry breaking in electroweak theory, Section 2.1.3, provided that the Higgs potentials have a non-zero vacuum expectation value, the fields could be expressed, at tree level, as shown in equation (2.65):

$$
\Phi_{1} \equiv\left\langle 0\left|\Phi_{1}\right| 0\right\rangle \equiv \frac{1}{\sqrt{2}}\left(\begin{array}{c}
v_{1} \\
0
\end{array}\right), \quad \Phi_{2} \equiv\left\langle 0\left|\Phi_{2}\right| 0\right\rangle \equiv \frac{1}{\sqrt{2}}\left(\begin{array}{c}
0 \\
v_{2}
\end{array}\right)
$$

\footnotetext{
${ }^{6}$ Due to cosmological constraints, a cold dark matter candidate need to be stable and neu$\operatorname{tral}[57,58]$.
} 
with $v_{1}$ and $v_{2}$ being the minimum Higgs potential such as

$$
v^{2}=v_{1}^{2}+v_{2}^{2}=\frac{4 M_{W}^{2}}{g^{2}}=(246 \mathrm{GeV})^{2}
$$

Moreover, these two parameters, $v_{1}$ and $v_{2}$, are directly related to the Higgs couplings to the down-type and up-type quarks $[40,59]$. The ratio of the the vacuum expectation values defines one of the main parameters of the Higgs sector

$$
\tan \beta=\frac{v_{2}}{v_{1}}
$$

While the two $\mathcal{C P}$-even neutral Higgs bosons derive from the real part of the mass matrix

$$
\left(\begin{array}{c}
\mathrm{h}^{0} \\
\mathrm{H}^{0}
\end{array}\right)=\sqrt{2}\left(\begin{array}{cc}
\cos \alpha & \sin \alpha \\
-\sin \alpha & \cos \alpha
\end{array}\right)\left(\begin{array}{c}
\operatorname{Re} \phi_{1}^{0 \star}-v_{1} \\
\operatorname{Re} \phi_{2}^{0 \star}-v_{2}
\end{array}\right),
$$

the $\mathcal{C P}$-odd neutral Higgs boson is obtained from the imaginary part

$$
\mathrm{A}^{0}=\sqrt{2}\left(-\operatorname{Im} \phi_{1}^{0 \star} \sin \beta+\operatorname{Im} \phi_{2}^{0 \star} \cos \beta\right)
$$

Finally, the charged Higgs bosons

$$
\mathrm{H}^{ \pm}=-\phi_{1}^{ \pm} \sin \beta+\phi_{2}^{ \pm} \cos \beta
$$

The mass of the charged Higgs bosons is the same, therefore the total number of free parameters is six, corresponding to the four masses, the mixing angle $\alpha$ and the ratio of the vacuum expectation values $\tan \beta$. At tree level, however only two of these parameters are relevant: $\tan \beta$ and one of the masses (customary $m_{\mathrm{A}^{0}}$ ) [60]. The other parameters can be derived from these two, according to the 
following equations

$$
\begin{aligned}
m_{\mathrm{H}^{ \pm}}^{2} & =m_{\mathrm{A}^{0}}^{2}+m_{\mathrm{W}}^{2} \\
m_{\mathrm{h}^{0}, \mathrm{H}^{0}}^{2} & =\frac{1}{2}\left(m_{\mathrm{A}^{0}}^{2}+m_{\mathrm{Z}}^{2} \pm \sqrt{\left(m_{\mathrm{A}^{0}}^{2}+m_{\mathrm{Z}}^{2}\right)^{2}-4 m_{\mathrm{Z}^{2}}^{2} m_{\mathrm{A}^{0}}^{2} \cos ^{2} 2 \beta}\right) \\
\tan 2 \alpha & =\tan 2 \beta\left(\frac{m_{\mathrm{A}^{0}}^{2}+m_{\mathrm{Z}}^{2}}{m_{\mathrm{A}^{0}}^{2}-m_{\mathrm{Z}}^{2}}\right)
\end{aligned}
$$

At large $\tan \beta, \mathrm{A}^{0}$ is almost degenerate in mass with either $\mathrm{h}^{0}$ or $\mathrm{H}^{0}$, Figure 2.6. For low mass $\mathrm{A}^{0}$, this boson is degenerate in mass with $\mathrm{h}^{0}$. For high mass, however, it is degenerate in mass with $\mathrm{H}^{0}$. The divide between these two regimes is around the $\mathrm{Z}^{0}$ mass pole, as can be read from equation (2.71). From the experimental point of view, this behavior doubles the effective sensitivity to find a neutral MSSM Higgs boson.

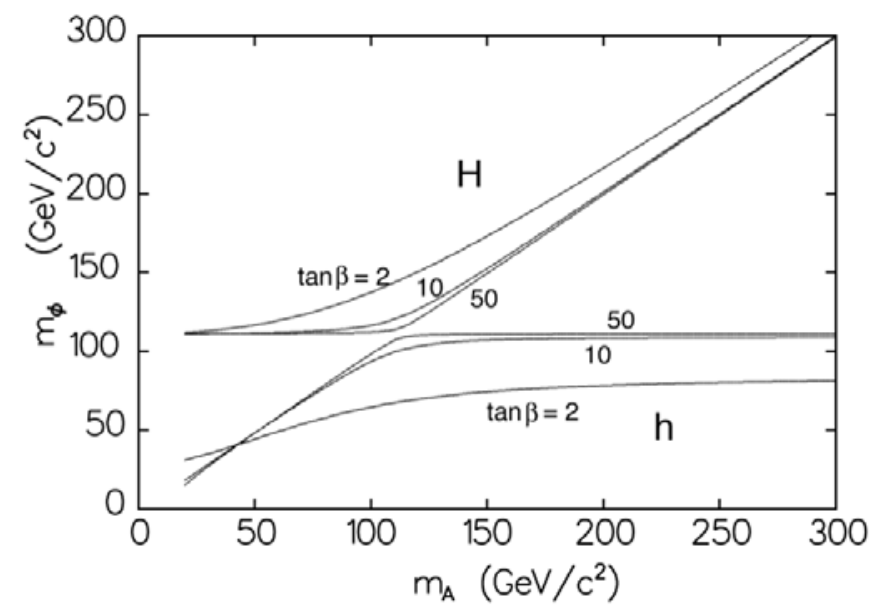

Figure 2.6: For large $\tan \beta$, the pseudoscalar boson $\mathrm{A}^{0}$ is degenerated in mass with either $\mathrm{h}^{0}$ or $\mathrm{H}^{0}$, referred as $\phi$

In this model, the couplings of two Higgs bosons that are degenerate in mass to up-type quarks is enhanced by a factor $\sim \tan ^{2} \beta$, while the third boson remains SM-like. This is a favorable scenario for the Tevatron, where the dominant production mechanisms are gluon fusion, mediated with a b-quark triangle, and $b \bar{b}$ 
annihilation, Figure 2.7. The Standard Model Higgs is produced by similar diagrams, but with $t$ quarks instead of $b$ quarks. The enhancement of the production diagrams with b-quarks by a factor of $\sim \tan ^{2} \beta$ beats the mass suppression factor $m_{b} / m_{t}$ that dominates the Standard Model cross sections. The production cross section enhancement at the Tevatron is very significant, as shown in Figure 2.8 and Figure 2.9.
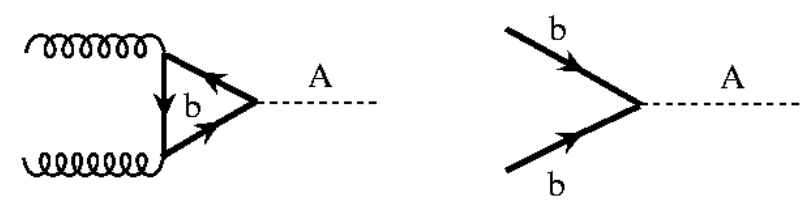

Figure 2.7: The main production mechanisms, at tree level, of neutral MSSM Higgs bosons is represented by these two diagrams. Gluon fusion or quark annihilation prefer b-quarks to t-quarks, at large $\tan \beta$ due to the coupling enhancement

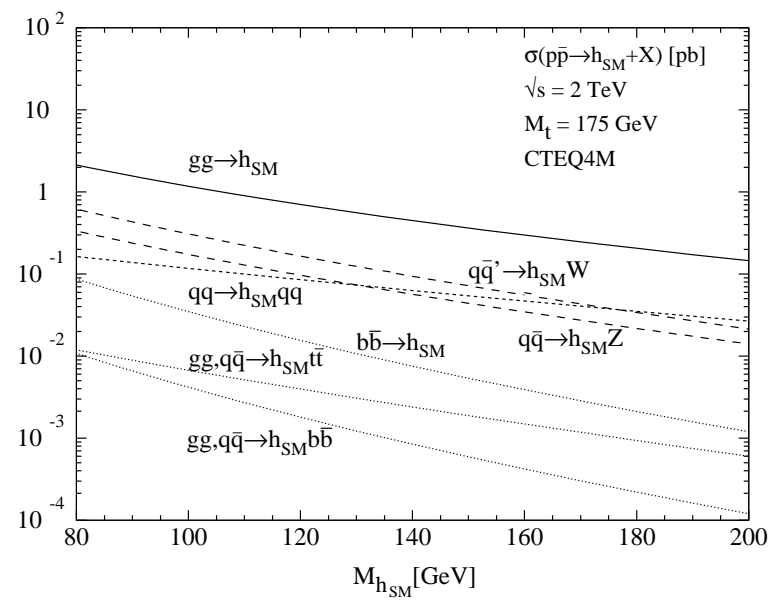

Figure 2.8: Standard Model Higgs boson production cross sections at the Tevatron, in pb.

The branching ratios of the Higgs bosons are, to first order, almost constant for the ranges of $\tan \beta$ and $m_{\mathrm{A}^{0}}$ of interest, [60]. A pair of b-quarks is the main decay mode, with a relative width of a $\sim 90 \%$. The remaining $\sim 10 \%$ is taken by the di-tau decay mode. The $\mathrm{b} \overline{\mathrm{b}}$ decay channel has a big advantage with respect to the di-tau channel in terms of branching ratio, Figure 2.10. However, given the nature of a hadron collider like the Tevatron where the $\mathrm{b} \overline{\mathrm{b}}$ production cross section via QCD processes is of the order of the $\mu \mathrm{b}$, a search for a mass resonance in this 


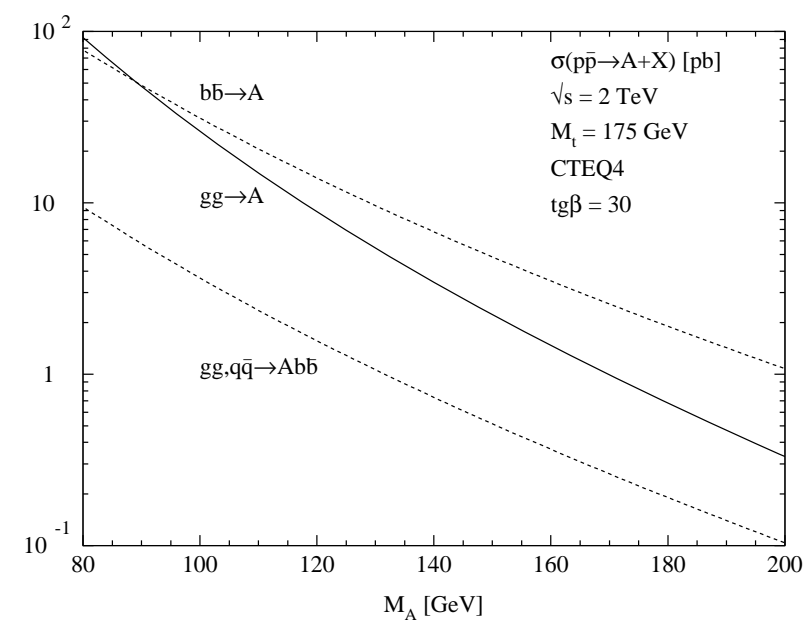

Figure 2.9: Pseudoscalar MSSM Higgs boson production cross sections at the Tevatron, in $\mathrm{pb}$, for $\tan \beta=30$.
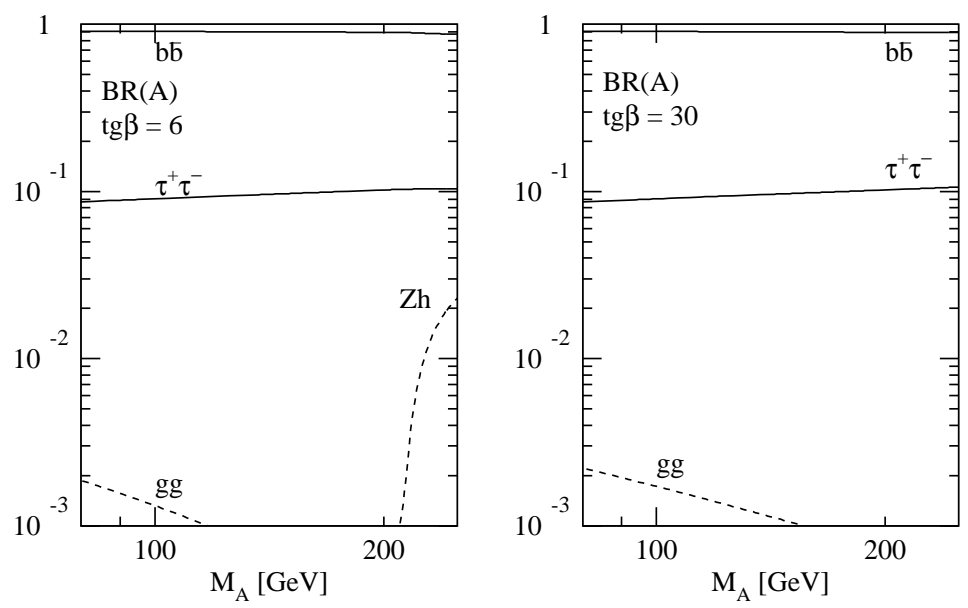

Figure 2.10: Pseudoscalar MSSM Higgs boson branching ratios, for $\tan \beta=6$ and $\tan \beta=30$. 
channel is experimentally inaccessible. With a smaller branching ratio, the di-tau decay channel competes against more rare electroweak processes, promoting this channel's sensitivity. Moreover, the level of understanding of electroweak boson and di-boson production is very well understood in CDF.

Tau leptons have a mean life of $c \tau=87.11 \mu s$. The leptonic decays, $\tau \rightarrow e \overline{\nu_{e}} \nu_{\tau}$ and $\tau \rightarrow \mu \overline{\nu_{\mu}} \nu_{\tau}$, account for $\sim 35 \%$ of the branching ratio [4]. The semi-hadronic decays involve more complicated signatures, with final states involving neutral and charged mesons. 


\section{Chapter 3}

\section{Experimental setup}

The Fermilab Tevatron is the highest energy hadron collider now in operation. After a major upgrade, the Tevatron Run II provides proton-antiproton $(p \bar{p})$ collisions with a center of mass energy of $1.96 \mathrm{TeV}$ and a bunch crossing period of 396 ns. Two detectors were desingned to extract the full scientific potential of these collisions: CDF, the Collider Detector at Fermilab and DØ. Both of them follow the usual structure of high energy physics experiments with a tracker, a calorimeter and a muon spectromenter, aranged in concentrical layers.

The results presented in the thesis make use of approximatelly $1.8 \mathrm{fb}^{-1}$ worth of data collected by CDF. A brief descrition of the accelerator chain and the detector is presented in the following sections. Also, a brief introduction to the measurment of luminosity in CDF as well as the trigger system is sketched.

\subsection{The Tevatron Collider}

The Tevatron Collider [61] located at the Fermi National Accelerator Laboratory (Fermilab) in Batavia (Illinois, USA) is a proton-antiproton $(p \bar{p})$ collider with a center-of-mass energy of $1.96 \mathrm{TeV}$. As shown in Figure 3.1, this complex has five major accelerators and storage rings used in successive steps, as is explained in 
detail below, to produce, store and accelerate the particles up to $980 \mathrm{GeV}$.

The acceleration cycle starts with the production of protons from ionized hydrogen atoms $H^{-}$, which are accelerated to $750 \mathrm{KeV}$ by a Cockroft-Walton electrostatic accelerator. Pre-accelerated hydrogen ions are then injected into the Linac where they are accelerated up to $400 \mathrm{MeV}$ by passing through a $150 \mathrm{~m}$ long chain of radio-frequency (RF) accelerator cavities. A carbon foil strips off the electrons of the $H^{-}$ions, thus producing protons. Inside the Booster the protons are merged into bunches and accelerated up to an energy of $8 \mathrm{GeV}$ prior to entering the Main Injector. In the Main Injector, a synchrotron with a circumference of $3 \mathrm{~km}$, the proton bunches are accelerated further to an energy of $150 \mathrm{GeV}$ and coalesced ${ }^{1}$ together before injection into the Tevatron.

\section{FERMILAB'S ACCELERATOR CHAIN}

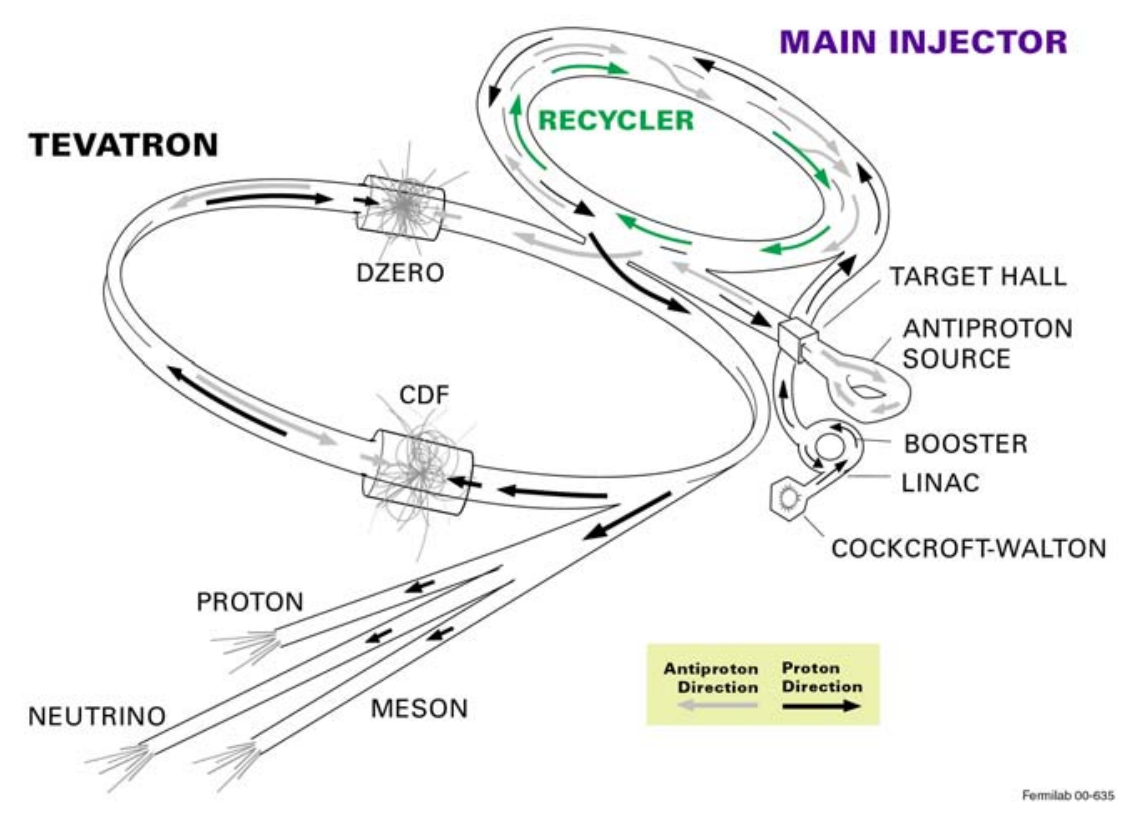

Figure 3.1: The Tevatron Collider Chain at Fermilab.

The production of the antiproton beam is significantly more complicated. The cycle starts with extracting a $120 \mathrm{GeV}$ proton beam from the Main Injector onto a

\footnotetext{
${ }^{1}$ coalescing is the process of merging proton bunches into one dense, high density beam
} 
stainless steel target. This process produces a variety of different particles, among which appear antiprotons ${ }^{2}$. The particles come off the target at many different angles and they are focused into a beam line with a Lithium lens. In order to select only the antiprotons, the beam is sent through a pulsed magnet which acts as a charge-mass spectrometer. The produced antiprotons are then injected into the Debuncher, an $8 \mathrm{GeV}$ synchrotron, which reduces the spread in the energy distribution of the antiprotons. After that, the antiproton beam is directed into the Accumulator, a storage ring in the Antiproton Source, where the antiprotons are stored at an energy of $8 \mathrm{GeV}$ and stacked to $10^{12}$ particles per bunch. The antiproton bunches are then injected into the Main Injector and accelerated to $150 \mathrm{GeV}$.

Finally, 36 proton and antiproton bunches are inserted into the Tevatron, a double acceleration ring of $1 \mathrm{~km}$ of radius, where their energy is increased up to 980 $\mathrm{GeV}$. Proton and antiproton bunches circulate around the Tevatron in opposite directions guided by superconducting magnets and where their orbits cross at the two collision points, B0 and D0. These interactions are observed by the CDF and $\mathrm{D} \emptyset$ detectors, respectively.

In the absence of a crossing angle or position offset, the luminosity at the interaction points is given by the expression:

$$
L=\frac{f_{b c} N_{b} N_{p} N_{\bar{p}}}{2 \pi\left(\sigma_{p}^{2}+\sigma_{\bar{p}}^{2}\right)} F\left(\frac{\sigma_{l}}{\beta^{*}}\right)
$$

where $f_{b c}$ is the revolution frequency, $N_{b}$ is the number of bunches, $N_{p(\bar{p})}$ is the number of protons (antiprotons) per bunch, and $\sigma_{p(\bar{p})}$ is the transverse and longitudinal rms proton (antiproton) beam size at the interaction point. $F$ is a form factor with a complicated dependence on beta function, $\beta^{*}$, and the bunch length, $\sigma_{l}$. The beta function is a measure of the beam width, and it is proportional to the beam's $x$ and $y$ extent in phase space. Table 3.1 shows the design Run II accelerator parameters [62].

Figure 3.2 and Figure 3.3 show, respectively, the evolution in the integrated luminosity, defined as $\mathcal{L}=\int L d t$, and the instantaneous luminosity delivered by

\footnotetext{
${ }^{2}$ The production rate, for $8 \mathrm{GeV}$ antiprotons, is about $18 \bar{p}$ per $10^{6} p$
} 


\begin{tabular}{l|c}
\hline \hline Parameter & Run II \\
\hline number of bunches $\left(N_{b}\right)$ & 36 \\
revolution frequency $[\mathrm{MHz}]\left(f_{b c}\right)$ & 1.7 \\
bunch rms $[\mathrm{m}] \sigma_{l}$ & 0.37 \\
bunch spacing $[\mathrm{ns}]$ & 396 \\
protons/bunch $\left(N_{p}\right)$ & $2.7 \times 10^{11}$ \\
antiprotons/bunch $\left(N_{\bar{p}}\right)$ & $3.0 \times 10^{10}$ \\
total antiprotons & $1.1 \times 10^{12}$ \\
$\beta^{*}[\mathrm{~cm}]$ & 35 \\
\hline \hline
\end{tabular}

Table 3.1: Accelerator parameters for Run II configuration.

Tevatron since the machine was turned on up to January 2008. The progressive increase in the integrated luminosity and the continuous records in the instantaneous luminosity ${ }^{3}$ prove the good performance of the accelerator.

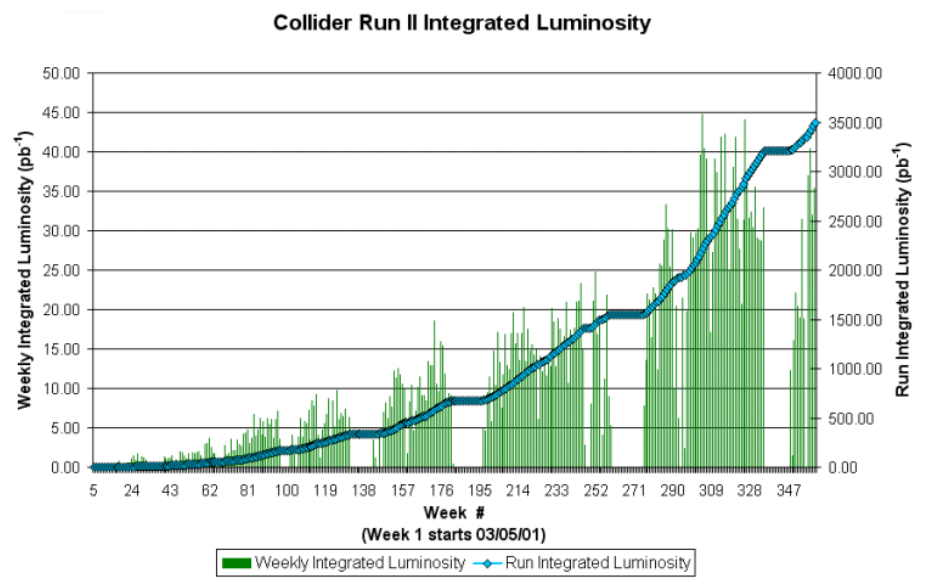

Figure 3.2: Tevatron Collider Run II Integrated Luminosity. The vertical green bar shows each week's total luminosity as measured in $\mathrm{pb}^{-1}$. The diamond connected line displays the integrated luminosity.

\footnotetext{
${ }^{3}$ As of January 2008, the record in the instantaneous luminosity was close to $3.0 \times 10^{32} \mathrm{~cm}^{-2} \mathrm{~s}^{-1}$.
} 


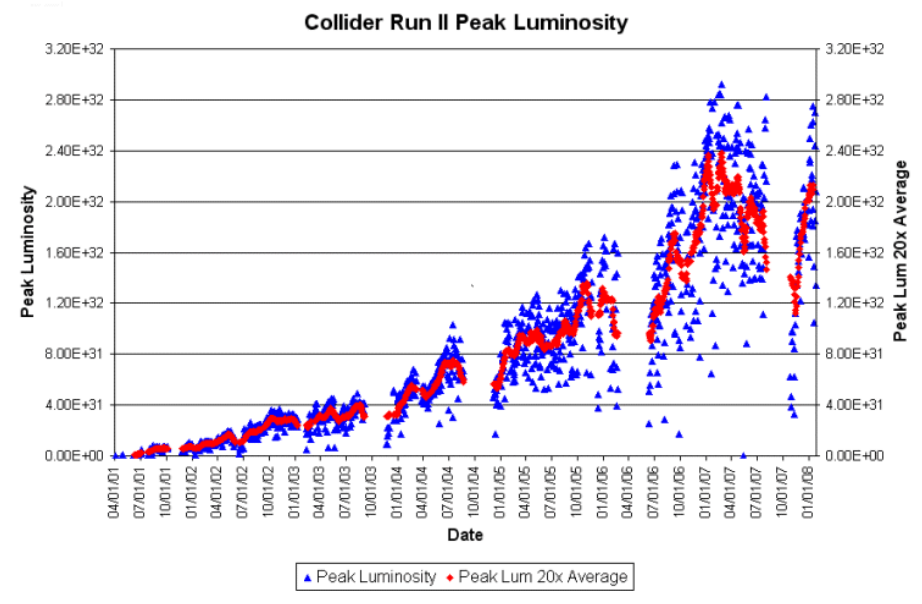

Figure 3.3: Tevatron Collider Run II Peak Luminosity. The blue squares show the peak luminosity at the beginning of each store and the red triangle displays a point representing the last 20 peak values averaged together.

\subsection{CDF Run II detector}

The CDF Run II detector [63], in operation since 2001, is an azimuthally and forward-backward symmetric apparatus designed to study $p \bar{p}$ collisions at the Tevatron. It is a general purpose, cylindrical-shaped detector which combines:

- A tracking system, that provides a measurement of the charged particle momenta, event $\mathrm{z}$ vertex position and detects secondary vertices.

- A Time-of-Flight system, to identify charged particles.

- A non-compensated calorimeter system, with the purpose of measuring the energy of charged and neutral particles produced in the interaction.

- Drift chambers and scintillators for muon detection.

The detector is shown in Figure 3.4 and Figure 3.5. CDF uses a coordinate system where the positive $z$-axis lies along the direction of the incident proton beam, $\phi$ is the azimuthal angle, $\theta$ is the polar angle (measured from the detector center), and $p_{\mathrm{T}}$ is the component of momentum in the transverse plane. A description of 


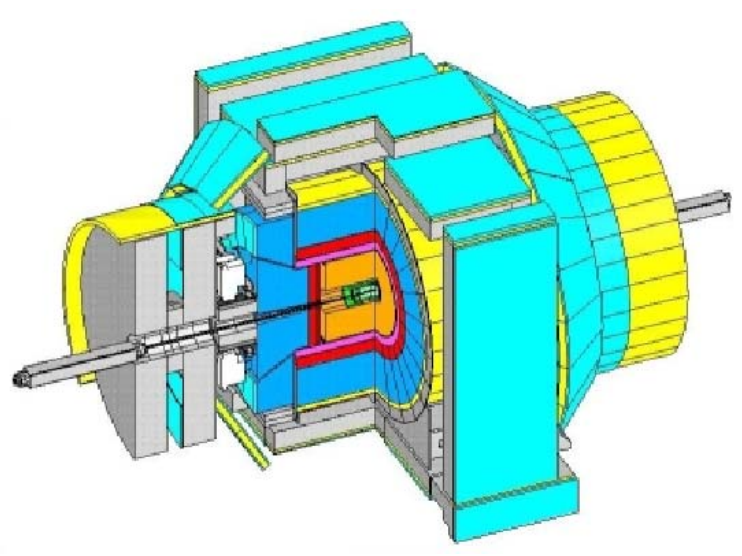

Figure 3.4: Isometric view of the CDF Run II detector.

all the systems starting from the devices closest to the beam and moving outward is presented in the next sections, where the detectors most relevant in the analysis are explained in more detail.

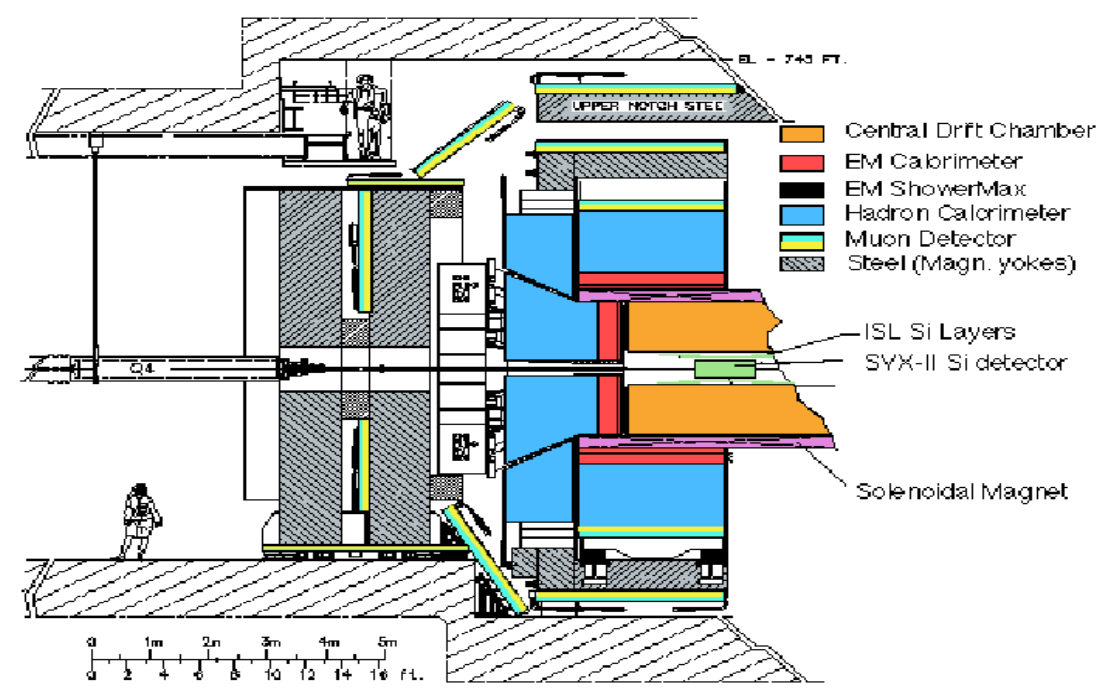

Figure 3.5: $r \times \eta$ side view of the CDF Run II detector. 


\subsubsection{Tracking and Time of Flight systems}

The tracking and time of flight systems are contained in a superconducting solenoid, $1.5 \mathrm{~m}$ in radius and $4.8 \mathrm{~m}$ in length, which generates a $1.4 \mathrm{~T}$ magnetic field parallel to the beam axis.

The part of the tracking system closest to the beam pipe is a silicon microstrip detector [64], which must be radiation-hard due its proximity to the beam. It extends from a radius of $1.5 \mathrm{~cm}$, the beam pipe, to $28 \mathrm{~cm}$, covering $|\eta|<2$ and has eight layers in a barrel geometry. The innermost layer is a single-sided silicon microstrip detector called Layer 00 (L00) which provides a $r \times \phi$ position measurement. The first five layers after the L00 constitute the Silicon Vertex Detector (SVXII) and the two outer layers comprise the Intermediate Silicon Layers system (ISL). These seven layers are made of double-sided silicon sensors, giving $r \times \phi$ and $z$ position information. The best position resolution achieved is $9 \mu \mathrm{m}$ in SVXII and the impact parameter resolution, including L00, arrives to $40 \mu \mathrm{m}$ at $p_{\mathrm{T}}>3 \mathrm{GeV} / \mathrm{c}$.

Surrounding the silicon detector is the Central Outer Tracker (COT) [65], the anchor of the CDF Run II tracking system. It is a $3.1 \mathrm{~m}$ long cylindrical drift chamber that covers the radial range from 40 to $137 \mathrm{~cm}(|\eta|<1)$. The COT contains 96 sense wire layers, which are radially grouped into eight "superlayers", as inferred from the end plate section shown in Figure 3.6.

Each superlayer is divided in $\phi$ into "supercells", and each supercell has 12 sense wires and a maximum drift distance that is approximately the same for all superlayers. Therefore, the number of supercells in a given superlayer scales approximately with the radius of the superlayer. The entire COT contains 30,240 sense wires. Approximately half the wires run along the $z$ direction ("axial"). The other half are strung at a small angle $\left(2^{\circ}\right)$ with respect to the $z$ direction ("stereo"). The combination of the axial and stereo information allows the measurement the $z$ positions. Particles originated from the interaction point, which have $|\eta|<1$, pass through all 8 superlayers of the COT.

The supercell layout, shown in Figure 3.7 for superlayer 2, consists of a wire plane containing sense and potential wires, for field shaping and a field (or cathode) 


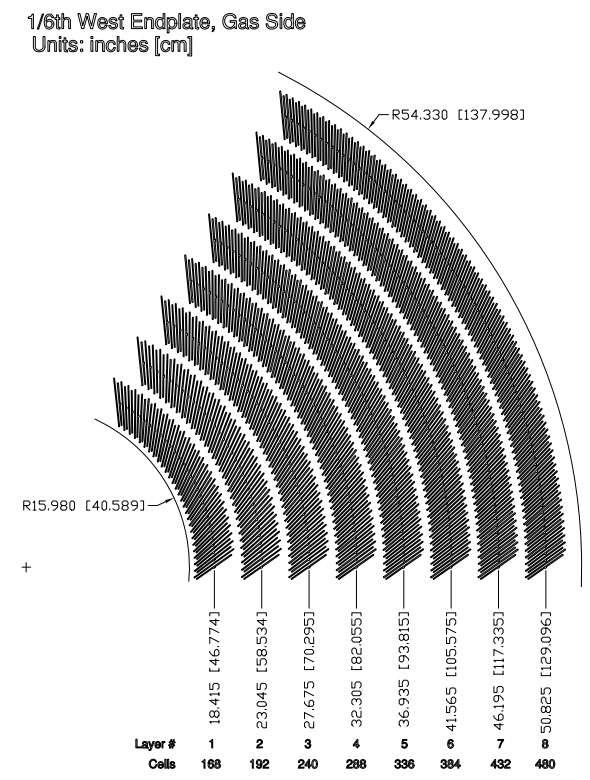

Figure 3.6: Layout of wire planes on a COT endplate.

sheet on either side. Both the sense and potential wires are $40 \mu \mathrm{m}$ diameter gold plated tungsten. The field sheet is $6.35 \mu \mathrm{m}$ thick Mylar with vapor-deposited gold on both sides. Each field sheet is shared with the neighboring supercell.

The COT is filled with an Argon-Ethane gas mixture and Isopropyl alcohol (49.5: $49.5: 1$ ). The mixture is chosen to have a constant drift velocity, approximately 50 $\mu \mathrm{m} / \mathrm{ns}$ across the cell width and the small content of isopropyl alcohol is intended to reduce the aging and build up of debris on the wires. When a charged particle passes through, the gas is ionized. Electrons drift toward the sense wires. Due to the magnetic field that the COT is immersed in, electrons drift at a Lorentz angle of $35^{\circ}$. The supercell is tilted by $35^{\circ}$ with respect to the radial direction to compensate for this effect. The momentum resolution of the tracks in the COT chamber depends on the $p_{\mathrm{T}}$ and is measured to be approximately $0.15 \%$, with corresponding hit resolution of about $140 \mu \mathrm{m}$ [66]. In addition to the measurement of the charged particle momenta, the COT is used to identify particles, with $p_{\mathrm{T}}>$ $2 \mathrm{GeV}$, based on $\mathrm{dE} / \mathrm{dx}$ measurements. 


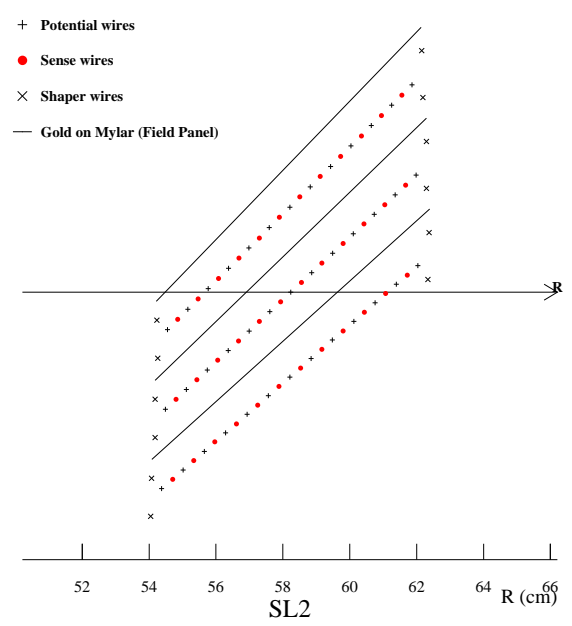

Figure 3.7: Layout of wires in a COT supercell.

Just outside the tracking system, CDF II has a Time of Flight (TOF) detector [67-69]. It consits on a barrel of scintillator, almost $3 \mathrm{~m}$ long, located at $140 \mathrm{~cm}$ from the beam line with a total of 216 bars, each covering $1.7^{\circ}$ in $\phi$ and pseudorapidity range $|\eta|<1$. Particle identification is achieved by measuring the time of arrival of a particle at the scintillators with respect to the collision time. Thus, combining the measured time-of-flight and the momentum and path length, measured by the tracking system, the mass of the particle can then determined. The resolution in the time-of-flight measurement has achieved $\approx 100 \mathrm{ps}$ and it provides at least two standard deviation separation between $K^{ \pm}$and $\pi^{ \pm}$ for momenta $\mathrm{p}<1.6 \mathrm{GeV} / c$.

As a summary, Figure 3.8 illustrates the Tracking and Time of Flight systems.

\subsubsection{Calorimeter system}

The calorimeter system surrounding the CDF tracking volume, outside of the solenoid coil. The different calorimeters that compose the system are scintillatorbased detectors, segmented in projective towers (or wedges), in $\eta \times \phi$ space, that point to the interaction region. The total coverage of the system is $2 \pi$ in $\phi$ and 


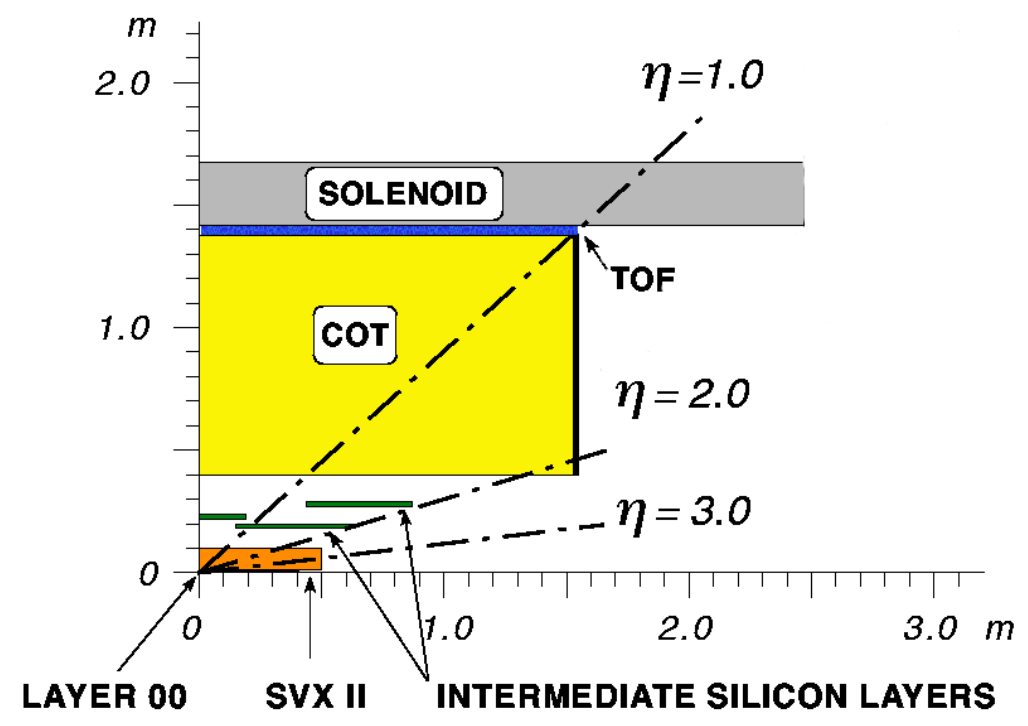

Figure 3.8: The CDF II tracker layout showing the different subdetector systems.

about $|\eta|<3.64$ units in pseudorapidity.

The calorimeter system is divided in two regions: central and plug. The central calorimeter covers the region $|\eta|<1.1$ and is split into two halves at $|\eta|=0$. It conceived as a hybrid system of sampling scitilators and strip wire proportional chambers. The forward plug calorimeters cover the angular range corresponding to $1.1<|\eta|<3.64$, as it is shown in Figure 3.9. Due to this structure, two "gap" regions are found at $|\eta|=0$ and $|\eta| \sim 1.1$.

\subsubsection{Central Calorimeters}

The central calorimeters consist of 478 towers, each one is $15^{\circ}$ in azimuth times approximately 0.11 in pseudorapidity. Each wedge consists of an electromagnetic component backed by a hadronic section. In the central electromagnetic calorimeter (CEM) [70], the scintillators are interleaved with lead layers. The total material 


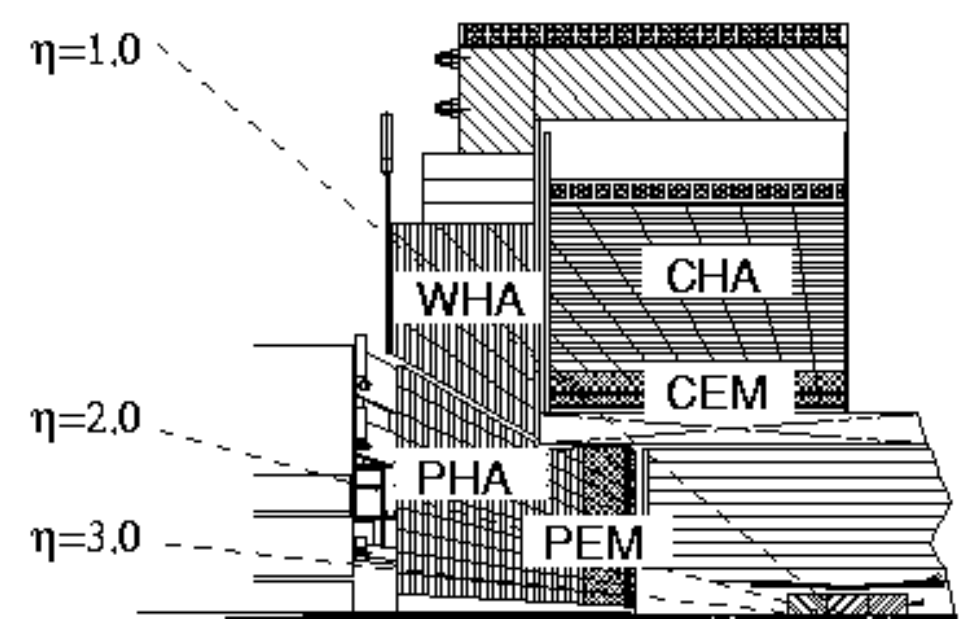

Figure 3.9: Elevation view of $1 / 4$ of the $\mathrm{CDF}$ detector showering the components of the CDF calorimeter: CEM, CHA, WHA, PEM and PHA.

has a depth of 18 radiation lengths $\left(X_{0}\right)^{4}$. The central hadronic section (CHA) [71] has alternative layers of steel and scintillator and is 4.7 interaction lengths deep $\left(\lambda_{0}\right)^{5}$. The endwall hadron calorimeter (WHA), with similar construction to $\mathrm{CHA}$, is located with half of the detector behind the CEM/CHA and the other half behind the plug calorimeter. The function of the WHA detector is to provide a hadronic coverage in the region $0.9<|\eta|<1$.3. In the central calorimeter the light from the scintillator is redirected by two wavelength shifting (WLS) fibers, which are located on the $\phi$ surface between wedges covering the same pseudorapidity region, up through the lightguides into two phototubes (PMTs) per tower.

The energy resolution for each section was measured in the testbeam and, for a

\footnotetext{
${ }^{4}$ The radiation length $X_{0}$ describes the characteristic amount of matter transversed, for highenergy electrons to lose all but $1 / e$ of its energy by bremsstrahlung, which is equivalent to $\frac{7}{9}$ of the length of the mean free path for pair $e^{+} e^{-}$production of high-energy photons. The average energy loss due to bremsstrahlung for an electron of energy $\mathrm{E}$ is related to the radiation length by $\left(\frac{d E}{d x}\right)_{b r e m s}=-\frac{E}{X_{0}}$ and the probability for an electron pair to be created by a high-energy photon is $\frac{7}{9} X_{0}$.

${ }^{5}$ An interaction length is the average distance a particle will travel before interacting with a nucleus: $\lambda=\frac{A}{\rho \sigma N_{A}}$, where $A$ is the atomic weight, $\rho$ is the material density, $\sigma$ is the cross section and $N_{A}$ is the Avogadro's number.
} 
perpendicular incident beam, it can be parameterized as:

$$
(\sigma / E)^{2}=\left(\sigma_{1} / \sqrt{E}\right)^{2}+\left(\sigma_{2}\right)^{2}
$$

where the first term comes from sampling fluctuations and the photostatistics of PMTs, and the second term comes from the non-uniform response of the calorimeter. In the CEM, the energy resolution for high energy electrons and photons is $\frac{\sigma\left(E_{\mathrm{T}}\right)}{E_{\mathrm{T}}}=\frac{13.5 \%}{\sqrt{E_{\mathrm{T}}}} \oplus 1.5 \%$, where $E_{\mathrm{T}}=E \sin \theta$, being $\theta$ the beam incident angle. Charge pions were used to obtain the energy resolution in the CHA and WHA detectors that are $\frac{\sigma\left(E_{\mathrm{T}}\right)}{E_{\mathrm{T}}}=\frac{50 \%}{\sqrt{E_{\mathrm{T}}}} \oplus 3 \%$ and $\frac{\sigma\left(E_{\mathrm{T}}\right)}{E_{\mathrm{T}}}=\frac{75 \%}{\sqrt{E_{\mathrm{T}}}} \oplus 4 \%$, respectively.

\subsubsection{Shower Maximum Detector}

The Electronmagnetic Calorimeter includes proportional wire strip chambers, the shower maximum detector (CES) [72,73]. These chambers are embeded in the calorimeter, at a depth equivalent to $5.9 X_{0}$. This is the point where the maximum deposition of energy of the shower is expected for a $15 \mathrm{GeV}$ electron [70]. The wires and strips in the CES run orthogonally and are digitized inpedependently, thus providing two dimensional information of the shower position and development.

The chambers are located at a distance of $184 \mathrm{~cm}$ perpendicular to the beam line. There are 64 golden-plated tungten wires running along the module, shileded by indivudual cells in a three piece aluminum extrusion. Each chamber is divided into two sections subsequent along the $z$-axis. A small inactive region is created where both regions meet.

The strips, made of copper, are attached to the aluminum extrusion with multiple layers of epoxy. The gain of the strips have been found to vary up to a $40 \%$ due to small distorsions of the shape. There are 128 strip, 69 in the section closer to the central plane of the dectector, and 59 in the outter section. In the later section, the strips are wider, thus fitting a smaller number in the same length.

This detector is instrumental for the reconstruction of hadronically decaying tau leptons, as it allows the definition of the impact position of $\pi^{0}$. Previous studies with photons have shown that the combination of the energy measurement of the

calorimeter towers and the positioning of the shower with the CES is capable of 


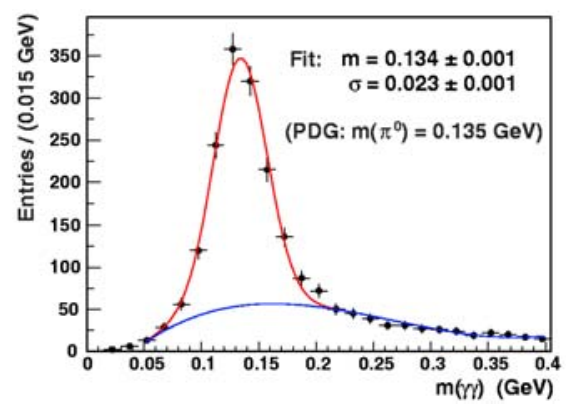

(a) Resolved photons in the same tower

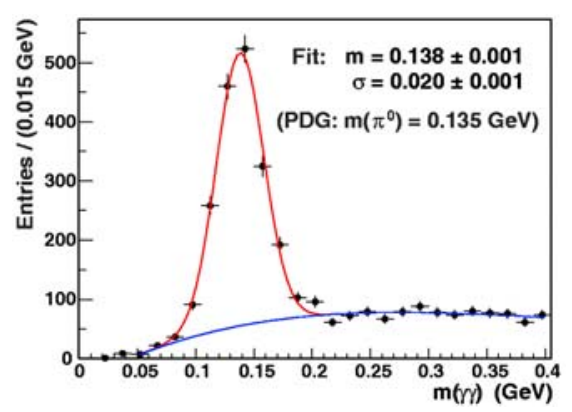

(b) Resolved photons in the different towers

Figure 3.10: Invariant mass of $\pi^{0}$ candidates, reconstructed from two resolved photons.

resolving photons from a $\pi^{0}$ decay, Figure 3.10, and even $\rho$ mesons, [74]. The CES also plays a very important roll in the trigger system, especially for electrons [75].

\subsubsection{Plug Calorimeters}

One of the major upgrades for the Run II was the plug calorimeter [76]. The new plug calorimeters are built with the same technology as the central components and replace the Run I gas calorimeters in the forward region. The $\eta \times \phi$ segmentation depends on the tower pseudorapidity coverage. For towers in the region $|\eta|<2.1$, the segmentation is $7.5^{\circ}$ in $\phi$ and from 0.1 to 0.16 in the pseudorapidity direction. For more forward wedges, the segmentation changes to $15^{\circ}$ in $\phi$ and about 0.2 to 0.6 in $\eta$.

As in the central calorimeters, each wedge consists of an electromagnetic (PEM) and a hadronic section (PHA). The PEM, with 23 layers composed of lead and scintillator, has a total thickness of about $21 X_{0}$. The PHA is a steel/scintillator device with a depth of about $7 \lambda_{0}$. In both sections the scintillator tiles are read out by WLS fibers embedded in the scintillator. The WLS fibers carry the light out to PMTs tubes located on the back plane of each endplug. Unlike the central calorimeters, each tower is only read out by one PMT. 
Testbeam measurements determined that the energy resolution of the PEM for electrons and photons is $\frac{\sigma}{E}=\frac{16 \%}{\sqrt{E}} \oplus 1 \%$. The PHA energy resolution is $\frac{\sigma}{E}=$ $\frac{80 \%}{\sqrt{E}} \oplus 5 \%$ for charged pions that do not interact in the electromagnetic component. Table 3.2 summarizes the calorimeter subsystems and their characteristics.

\begin{tabular}{|c|c|c|c|}
\hline Calorimeter & Coverage & Thickness & Energy resolution $(\mathrm{E}$ in $\mathrm{GeV})$ \\
\hline$\overline{\mathrm{CEM}}$ & $|\eta|<1.1$ & $18 X_{0}$ & $\frac{13.5 \%}{\sqrt{E_{\mathrm{T}}}} \oplus 2 \%$ \\
\hline CHA & $|\eta|<0.9$ & $4.7 \lambda_{0}$ & $\frac{50 \%}{\sqrt{E_{\mathrm{T}}}} \oplus 3 \%$ \\
\hline WHA & $0.9<|\eta|<1.3$ & $4.7 \lambda_{0}$ & $\frac{75 \%}{\sqrt{E_{\mathrm{T}}}} \oplus 4 \%$ \\
\hline$\overline{\mathrm{PEM}}$ & $1.1<|\eta|<3.6$ & $21 X_{0}, 1 \lambda_{0}$ & $\frac{16 \%}{\sqrt{E}} \oplus 1 \%$ \\
\hline PHA & $1.2<|\eta|<3.6$ & $7 \lambda_{0}$ & $\frac{80 \%}{\sqrt{E}} \oplus 5 \%$ \\
\hline
\end{tabular}

Table 3.2: CDF II Calorimeter subsystems and characteristics. The energy resolution for the EM calorimeter is given for a single incident electron and that for the hadronic calorimeter for a single incident pion.

The forward calorimeter also has a shower profile detector, the Plug Shower Maximum (PES), equivalent to the CES in the central region. Also the Central Preradiator (CPR) and the Plug Preradiator (PPR) are located at the inner face of the calorimeters, and are also used in particle identification, separating $e^{ \pm}, \gamma$ and $\pi^{0}$ and jets.

\subsubsection{Muons system}

The muon system, which consists of sets of drift chambers and scintillators, is installed beyond the calorimetry system as the radially outermost component of CDF Run II detector $(\mathrm{r} \sim 3.5 \mathrm{~m})$. The muon system [77] is divided into different subsystems: the Central Muon Detector, CMU, the Central Muon Upgrade Detector, CMP, the Central Muon Extension Detector, CMX, and the Intermediate Muon Detector, IMU.

The coverage of the muon systems is almost complete in phi, except some gaps, and spans in polar angle up to $|\eta| \approx 1.5$, Figure 3.11. Attached to the calorimeter modules, the CMU consists of a stack of 4 layers of drift chambers. The different 
layers are slightly shifted in $p h i$ for better performance. These chambers are singlewired and the read-out is equiped with a TDC and an ADC at each end of the wire. The $\phi$-position is then calculated from the dirft time, measured with the TDC, while the hit $z$-positio is found through harge division with the ADC.

The central muon upgrade detector, CMP, forms a box around the detector of stacked drift chambers. A layer of $60 \mathrm{~cm}$ of steel, partially used for the magnetic field return, provides the needed shielding to absorb particles, other than muons, leaking the calorimeter. This system overlaps with the CMU, and covers the central part

Beyond the CMU and CMP detectors, the central muon extension detector, CMX, This detector consists of stacked cells of drift tubes conforming a conical section. The chambers are stacked at a small angle, allowing for polar angle mesurement. Given the space constrains in the collision hall, the coverage is not complete in $\phi$.

The main component of the IMU are the Barrel Chambers (BMU). This detector is shaped as two contigous barrels of dift chambers located on the outer radius of the toroids. These chambers expand the muon coverage of CDF up to $|\eta| \approx 1.5$, but on cover the upper 270deg in azimuth.

Sets of scintillators were also installed for triggering and spurious signal rejection. The central muon scintillator upgrade, CSP, are counters installed on the outer surface of the CMP chambers. Two layers of scintillators are mounted on the internal and external sides of the CMX, the so-called central muon extension scintillator, CSX. Finally, the IMU incorporates two scintillator systems: the barrel scintillator upgrade, BSU, and the Toroid Scintillator Upgrade, TSU. The BSU detector is made of rectangular scintillators mounted on the outside of the BMU chambers and with the same azimuthal coverage. The TSU detector is made of trapezoidal scintillators mounted on the inner face of the toroid and covering $2 \pi$ in azimuth. 


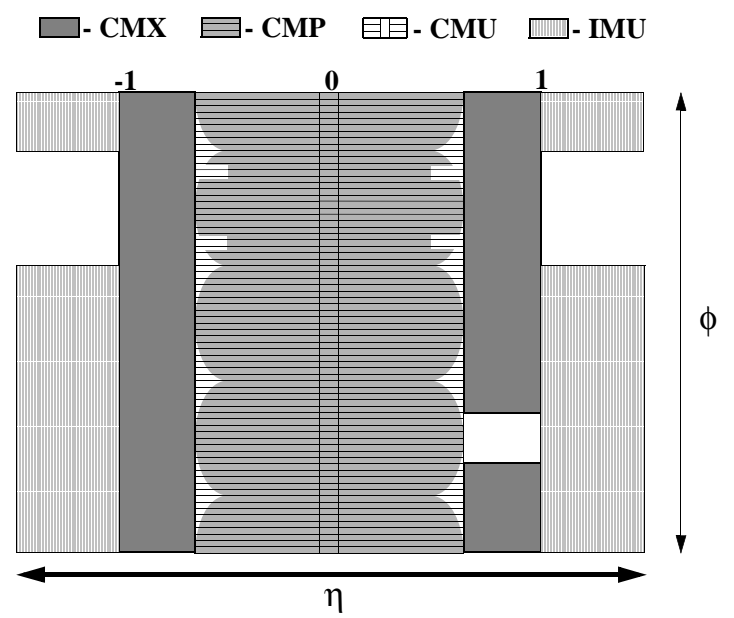

Figure 3.11: $\eta$ - $\phi$ coverage of the different muon subsystems: central muon detector (CMU), central muon upgrade detector (CMP), central muon extension (CMX), and the intermediate muon detector (IMU). The IMU includes the barrel chambers (BMU) and some scintillators.

\subsection{Luminosity Measurement}

\subsubsection{CLC detector}

In CDF, the beam luminosity is determined using gas Čerenkov counters (CLC) [78] located in the pseudorapidity region $3.7<|\eta|<4.7$, which measure the average number of inelastic interaction per bunch crossing. Each module consists of 48 thin, gas-filled, Čerenkov counters. The counters are arranged around the beam pipe in three concentric layers, with 16 counters each, and pointing to the center of the interaction region. The cones in the two outer layers are about $180 \mathrm{~cm}$ long and the inner layer counters, closer to the beam pipe, have a length of $110 \mathrm{~cm}$. The Čerenkov light is detected with photomultiplier tubes located at the end of the tubes, Figure 3.12 . 


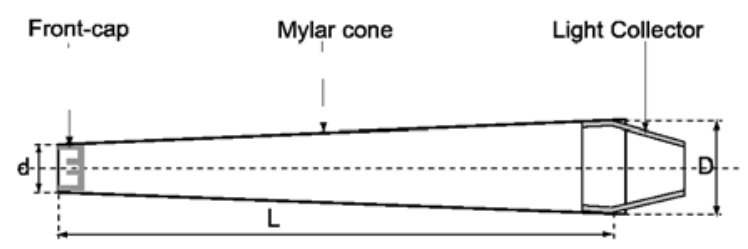

Figure 3.12: Schematic drawing of a cone of the Čerenkov luminosity counters, CLC. An aluminum light collector directs the light reflected in the mylar cone to the photomultiplier, PMT, attached at the end of the tube.

\subsubsection{Measurement of the luminosity}

The average number of primary interactions, $\mu$, is related to the instantaneous luminosity, $\mathcal{L}$, by the expression:

$$
\mu \cdot f_{b c}=\sigma_{t o t} \cdot \mathcal{L}
$$

where $f_{b c}$ is the bunch crossings frequency at Tevatron, on average $1.7 \mathrm{MHz}$ for $36 \times 36$ bunch operations, and $\sigma_{t o t}$ is the total $p \bar{p}$ cross section.

Since the CLC is not sensitive at all to the elastic component of the $p \bar{p}$ scattering, the equation (3.3) can be rewritten using the inelastic cross section, $\sigma_{i n}$, as

$$
\mathcal{L}=\frac{\mu \cdot f_{b c}}{\sigma_{\text {in }}},
$$

where now $\mu$ is the average number of inelastic $p \bar{p}$ interactions. The method used in CDF for the luminosity measurement is based on the counting of empty crossings [79]. This method determines $\mu$ by measuring the first bin of the distribution which corresponds to the probability of having zero inelastic interactions, $P_{0}$, through the relation: 


$$
P_{0}(\mu)=e^{-\mu}
$$

which is correct if the acceptance of the detector and its efficiency were $100 \%$. Given the limited extent of this statement, there are some selection criteria, $\alpha$, to define an "interaction". An "interaction" is defined as a $p \bar{p}$ crossing with hits above a fixed threshold on both sides of the CLC detector. Therefore, an empty crossing is a $p \bar{p}$ crossing with no interactions. Given these selection criteria, the experimental quantity $P_{0}$, called $P_{0}^{e x p}\{\alpha\}$, is related to $\mu$ as:

$$
P_{0}^{\exp }\{\mu ; \alpha\}=\left(e^{\epsilon_{\omega} \cdot \mu}+e^{-\epsilon_{e} \cdot \mu}-1\right) \cdot e^{-\left(1-\epsilon_{0}\right) \cdot \mu},
$$

where the acceptances $\epsilon_{0}$ and $\epsilon_{\omega / e}$ are, respectively, the probability to have no hits in the combined east and west CLC modules and the probability to have at least one hit exclusively in west/east CLC module. The evaluation of these parameters is based on Monte Carlo simulations, and typical values are $\epsilon_{0}=0.07$ and $\epsilon_{\omega / e}=0.12$.

To obtain the luminosity measurement using the equation (3.4), the value of $\sigma_{i n}$ is still needed. At the beginning of Run II, an extrapolation to $2 \mathrm{TeV}$ of the value measured at $\sqrt{s}=1.8 \mathrm{TeV}$ by $\mathrm{CDF}[80]$ was used. The cross section would be $\sigma_{i n}=60.4 \mathrm{mb}$. To facilitate the comparison of $\mathrm{CDF}$ and $\mathrm{D} \varnothing$ cross section measurements in Run II, the collaborations agreed to use a common inelastic cross section [81], $\sigma_{i n}=59.3 \mathrm{mb}$ that is about $1.9 \%$ smaller than previous value. Since CDF never modified the value used online and offline, the CDF quoted luminosity is multiplied offline by a factor of 1.019 .

Different sources of uncertainties have been taken into account to evaluate the systematic uncertainties on the luminosity measurement [82]. The dominated contributions are related to the detector simulation and the event generator used, and have been evaluated to be about $3 \%$. The total systematic uncertainty in the CLC luminosity measurements is $5.8 \%$, which includes uncertainties on the measurement, $4.2 \%$, and on the inelastic cross section value, $4 \%$. 


\subsection{Trigger and Data Acquisition}

The average interaction rate at the Tevatron is $1.7 \mathrm{MHz}$ for $36 \times 36$ bunches. In fact, the actual interaction rate is higher because the bunches circulate in three trains of 12 bunches in each group spaced 396 ns which leads to a crossing rate of $2.53 \mathrm{MHz}$. The interaction rate is orders of magnitude higher than the maximum rate that the data acquisition system can handle. Furthermore, the majority of collisions are not of interest. This leads to implementation of a trigger system that preselects events online and decides if the corresponding event information is written to tape or discarded.

The CDF trigger system consists of three trigger levels, see Figure 3.13 and Figure 3.14. The first two levels are hardware based, while the third one consists on a processor farm. The decisions taken by the system are based on increasingly more complex event information. The two hardware levels are monitored and controlled by the Trigger Supervisor Interface, TSI, which distributes signals from the different sections of the trigger and DAQ system, a global clock and bunch crossing signal. 


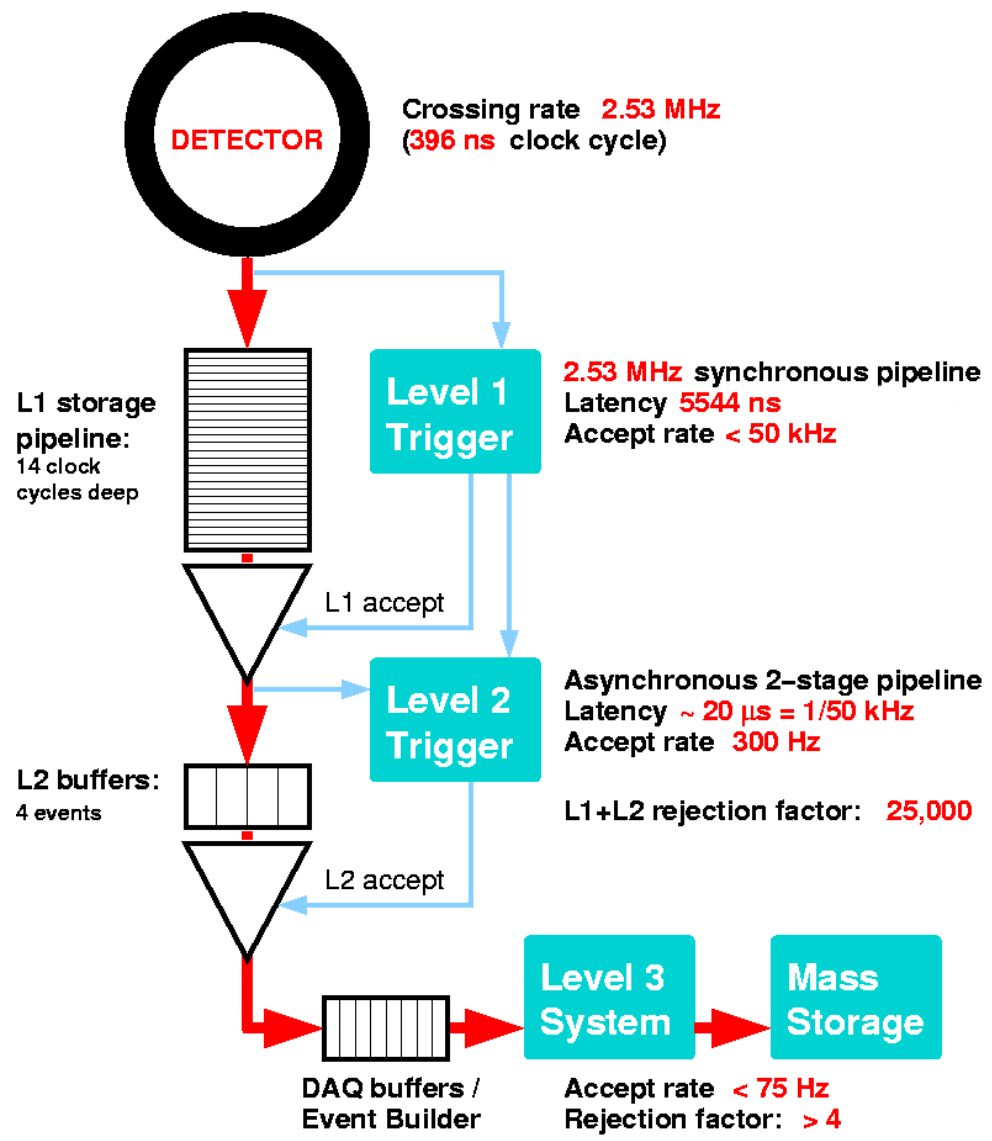

Figure 3.13: Block diagram showing the global trigger and DAQ systems at CDF II. 


\subsubsection{Level 1 trigger}

The Level 1 trigger is a synchronous system that reads events and takes a decision every beam crossing. The depth of the $\mathrm{L} 1$ decision pipeline is approximately $4 \mu \mathrm{s}$, L1 latency. The L1 buffer must be at least as deep as this processing pipeline or the data associated with a particular L1 decision would be lost before the decision is made. The L1 buffer is 14 crossings deep (5544 ns at $396 \mathrm{~ns}$ bunch spacing) to provide a margin for unanticipated increases in L1 latency. The Level 1 reduces the event rates from $2.53 \mathrm{MHz}$ to less than $50 \mathrm{kHz}$.

The Level 1 hardware consists of three parallel processing streams which feed inputs of the Global Level 1 decision unit. One stream finds calorimeter based objects, L1 CAL, another finds muons, L1 MUON, while the third one finds tracks in the COT, L1 TRACK. Since the muons and the calorimeter based objects require the presence of a track pointing at the corresponding outer detector element, the tracks must be sent to the calorimeter and muon streams as well as the track only stream.

- The L1 CAL calorimeter trigger is employed to detect electrons, photons, jets, total transverse energy and missing transverse energy, $E_{\mathrm{T}}^{\mathrm{miss}}$. The calorimeter triggers are divided into two types: object triggers (electron, photons and jets) and global triggers $\left(\sum E_{\mathrm{T}}\right.$ and $\left.E_{\mathrm{T}}^{\text {miss }}\right)$. The calorimeter towers are summed into trigger towers of $15^{\circ}$ in $\phi$ and by approximately 0.2 in $\eta$. Therefore, the calorimeter is divided in $24 \times 24$ towers in $\eta \times \phi$ space [83]. The object triggers are formed by applying thresholds to individual calorimeter trigger towers, while thresholds for the global triggers are applied after summing energies from all towers.

- The L1 TRACK trigger is designed to detect tracks on the COT. An eXtremely Fast Tracker, XFT, [84] uses hits from 4 axial layers of the COT to find tracks with a $p_{\mathrm{T}}$ greater than some threshold, $\sim 2 \mathrm{GeV} / \mathrm{c}$. The resulting track list is sent to the extrapolation box, XTRP, $[85]$ that distributes the tracks to the Level 1 and Level 2 trigger subsystems.

- L1 MUON system uses muon primitives, generated from various muon detector elements, and XFT tracks extrapolated to the muon chambers by the 
XTRP to form muon trigger objects. For the scintillators of the muon system, the primitives are derived from single hits or coincidences of hits. In the case of the wire chambers, the primitives are obtained from patterns of hits on projective wire with the requirement that the difference in the arrival times of signals be less than a present threshold. This maximum allowed time difference imposes a minimum $p_{\mathrm{T}}$ requirement for hits from a single tracks.

Finally, the Global Level 1 makes the L1 trigger decision based on the objects of interest found by the different Level 1 processes. Different sets of Level 1 conditions are assigned to the Level 1 trigger bits. If these conditions are met, the the bit is set to true. All this information is later hadled the the TSI and transfered to the other trigger levels, and eventually, to tape.

\subsubsection{Level 2 trigger}

The Level 2 trigger is an asynchronous system which processes events that have received a L1 accept in FIFO (First In - First Out) manner. It is structured as a two-stage pipeline with data buffering at the input of each stage. The first stage is based on a dedicated hardware processor which assembles information from a particular section of the detector. The second stage consists of a programmable processors operating on lists of objects generated by the first stage. Each of the L2 stages is expected to take approximately $10 \mu$ s giving a latency of approximately $20 \mu \mathrm{s}$. The L2 buffers provide a storage of four events. After the Level 2, the event rate is reduced to about $300 \mathrm{~Hz}$.

In addition of the trigger primitives generated for L1, data for the L2 come from the shower maximum strip chambers in the central calorimeter and the $r \times \phi$ strips of the SVX II. There are three hardware systems generating primitives at Level 2: Level 2 cluster finder, L2CAL, shower maximum strip chambers in the central calorimeter, XCES, and the Silicon Vertex Tracker (SVT).

- The L2CAL hardware carries out the hardware cluster finder functions. It receives trigger tower energies from the L1 CAL and applies seed and "shoulder" thresholds for cluster finding. It is basically designed for triggering on 
jet.

- The shower maximum detector provides a much better spacial resolution than a calorimeter tower. The XCES boards perform sum of the energy on groups of four adjacent CES wires and compare them to a threshold (around $4 \mathrm{GeV}$ ). This information is matched to XFT tracks to generate a Level 2 trigger. This trigger hardware provides a significant reduction in combinatorial background for electrons and photons.

- Silicon Vertex Tracker, SVT, [86] uses hits from the $r \times \phi$ strips of the SVX II and tracks from the XFT to find tracks in SVX II. SVT improves on the XFT resolution for $\phi$ and $p_{\mathrm{T}}$ and adds a measurement of the track impact parameter, $d_{0}$. Hereby the efficiency and resolution are comparable to those of the offline track reconstruction. The SVT enables triggering on displaced tracks, that have a large $d_{0}$.

When the objects reconstructed by the Level 2 processors meet the conditions stated in the trigger table for the Level 2, the event is assigned the corresponding Level 2 trigger bit, provided that the corresponding Level 1 bit is already set. Now, the TSI sends the event to the Level 3 farm. 


\section{RUN II TRIGGER SYSTEM}

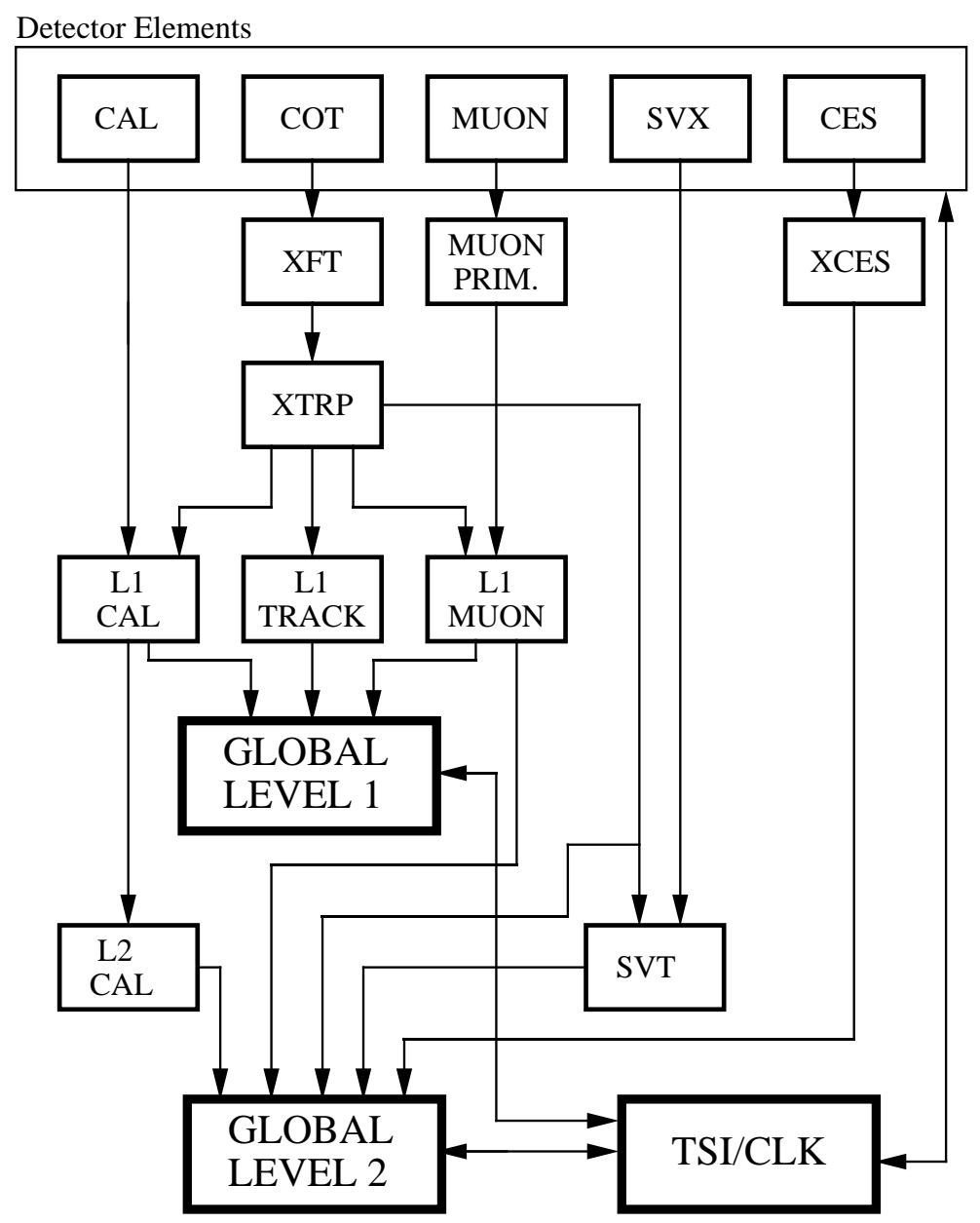

PJW 9/23/96

Figure 3.14: Block diagram showing the Level 1 and Level 2 trigger systems. 


\subsubsection{Level 3 trigger}

After an event is accepted at Level 2, it has to be read out completelly. This operation involves collecting data from over a couple of hundreds of VME Readout Buffers (VRBs). The purpose of the Event Builder is assembling the event from pieces of data from the L2 system into complete events. It is divided into 16 subfarms, each consisting of 12 to 16 processor nodes. Once the event is built, it is sent to one node in the Level 3 farm. The Level 3 trigger reconstructs the event following given algorithms. These algorithms take advantage of the full detector information and improved resolution not available to the lower trigger levels. This includes a full 3-dimensional track reconstruction and tight matching of tracks to calorimeter and muon-system information. Events that satisfy the Level 3 trigger requirements are then transfered onward to the Consumer Server/Data Logger (CSL) system for storage first on disk and later on tape. The average processing time per event in Level 3 is on the order of a few seconds. The Level 3 leads to a further reduction in the output rate, roughly $50 \mathrm{~Hz}$.

A set of requirements that an event has to fulfill at Level 1, Level 2 and Level 3 constitutes a trigger path. The CDF II trigger system implements about 200 trigger paths. An event will be accepted if it passes the requirements of any one of these paths and, depending of the trigger path, it will be stored in a trigger dataset. A complete description of the different datasets at CDF Run II can be found in [87].

Another important feature of the tirgger system of CDF is that Level 2 accepts can be prescaled. This means that only a fraction of the events that fullfil the trigger requirements are actually accepted. Even if this implies loosing potential good events, it becomes necessary at high luminosity. Given the continously improving performance of the Tevatron, prescaling trigger has become common practice in the last years. Moreover, the trigger system allows for dynamic prescaling of trigger accepts, meaning that the scaling factor varies with the instantaneous luminosity, so the output bandwidth is maximally utilized. 


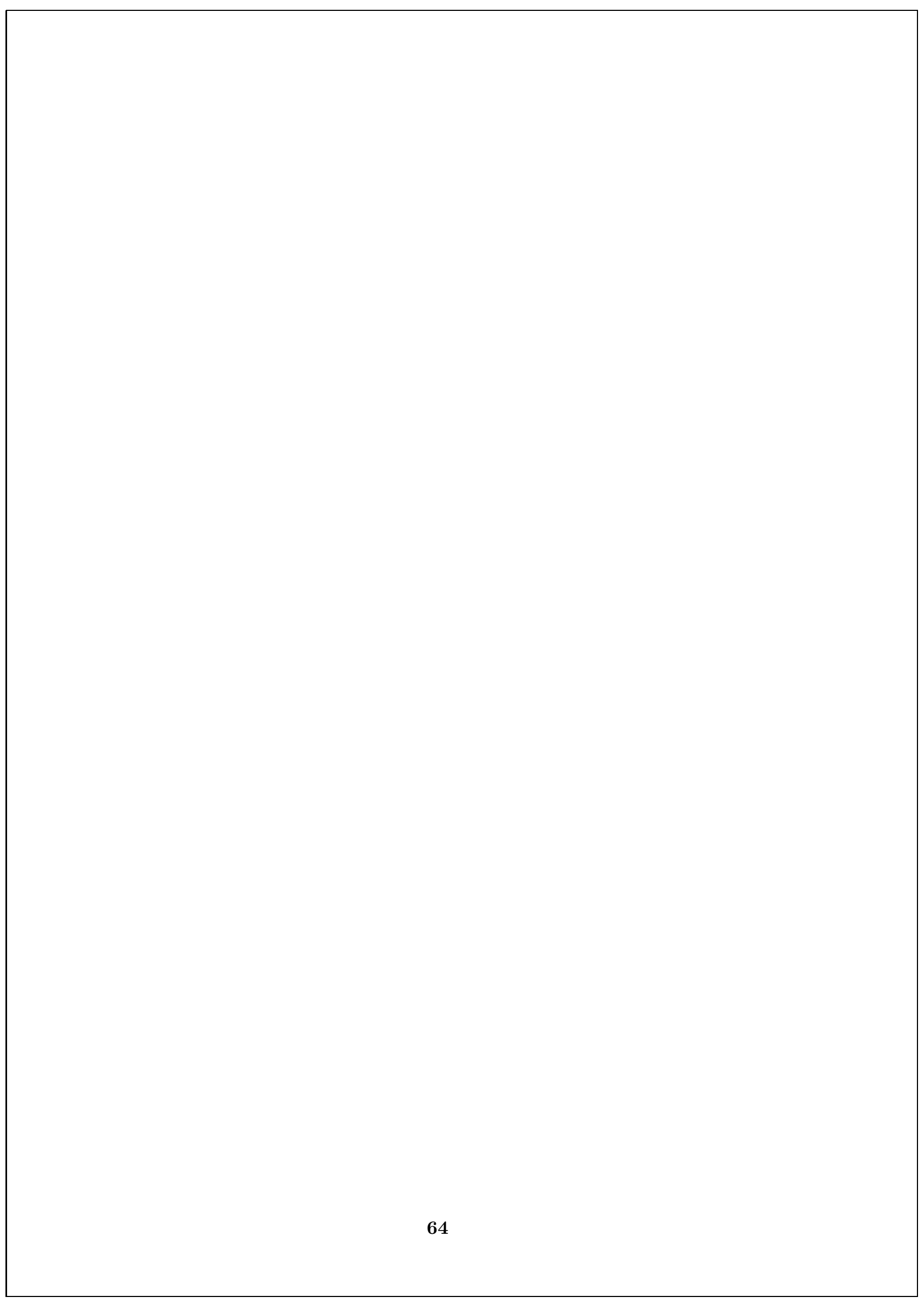




\section{Chapter 4}

\section{Data Samples and Simulation}

As stated in Chapter 1, this analysis combines three different final states: $\tau_{e} \tau_{\mathrm{h}}$, $\tau_{\mu} \tau_{\mathrm{h}}$ and $\tau_{e} \tau_{\mu}$, where $\tau_{e}$ represents a tau lepton decaying to an electron, $\tau_{\mu}$ represents a tau lepton decaying to a muon and $\tau_{\mathrm{h}}$ represents a tau decaying hadronically.

The data is collected for each channel uses different trigger requirements, so-called trigger paths in CDF. The trigger requirements introduce inefficiencies that have to be taken into account when comparing real data vs simulation distributions.

The analysis described in the following chapters is based on $\sim 1.8 \mathrm{fb}^{-1}$ of data, collected from almost the beginning of RunII and up to the end of March 2007. In CDF nomenclature, this correspond to the data taken from period 0 to period 11 , runs 138425 to 237795 . This period of time spans over several years, in which the definition of the trigger paths evolved. This evolution included the addition of pre-scaling of the L2 trigger and the disabling of some trigger paths above some luminosities. For this reason, the integrated luminosities over this period of datataking varies for different trigger paths. 


\subsection{Data}

For the exploration of the $\tau_{e} \tau_{\mu}$ channel we use data collected with the "SUSY dilepton" trigger paths: datasets edilad, edilbh, edilai, edilaj. The data is taken from the officially produced Stntuple samples.

The "SUSY dilepton" triggers select two leptons $(l=e, \mu)$ with with $p_{T}>4 \mathrm{GeV}$ (during period $i$, the threshold for one of the leptons was increased to $8 \mathrm{GeV}$ ). At least one of the leptons is in the central region. In this analysis we use only the trigger paths that select one electron and one muon, both in the central detector region. Same-flavor leptons are used for consistency checks and study of lepton isolation consistency between the data and MC.

The same triggers are used in several of the trilepton SUSY searches. We use the efficiency for the electron leg of the trigger from parallel studies [88]. After a turn on, it reaches a constant value of $\sim 0.96$ for electrons above $10 \mathrm{GeV}$. The trigger efficiencies for the muon leg of the trigger are well understood in CDF [89]. In the high- $E_{\mathrm{T}}\left(p_{\mathrm{T}}\right)$ ranges the results for both legs agree with the JP values obtained for the inclusive electron(muon) samples.

For the final states with a hadronically decaying tau we use the "lepton+track" triggers that require a central electron or muon, and an isolated track (used as a starting point for tau reconstruction).

The "track" (tau) leg of the "lepton+track" triggers is described in the Chapter 5 , where we also present the results of the efficiency studies.

For the trigger efficiency of the muon leg we use the results for the high- $p_{T}$ muon trigger - except for the $p_{T}$ threshold the requirements are the same. Our muon trigger efficiency studies in 5.3 .3 data show that for muons with $p_{T}>10 \mathrm{GeV}$ we are at the plateau of the efficiency, and that it is consistent with the results for the high- $p_{T}$ muons in the region $p_{T}>20 \mathrm{GeV}$. The trigger efficiency for the electron leg is obtained with the same samples and procedures as for the SUSY dilepton trigger, and uses the same parametrization.

The overall trigger efficiencies for our final states are product of the efficiencies of the two legs used in event selection. They are applied to the MC samples by 
assigning a weights to each selected MC event event.

In this analysis we use Run 2 data in the run range 150145-2 and apply the Top/EWK/Exotics good run list v17 (good electron, muon, no Si requirement, excluded COT compromised runs). This run range has both the CMX and CMU+CMP systems operational. The integrated luminosity of our sample is $\sim 1.8 \mathrm{fb}^{-1}$.

\subsection{Monte Carlo}

Simulation is a very powerful tool to predict the composition of the final state distributions and the contributions of not-yet-observed processes. Moreover, reconstruction and identification efficiencies as well as event acceptance calculation rely on an accurate performance of the simulation. Monte Carlo, MC, techniques are used to simulate $\mathrm{p} \overline{\mathrm{p}}$ collisions and the signal read out by the CDF. The collisions are generated using PYTHIA [90] and the interaction of the collision products with the detector is simulated with GEant3 [91]. A special package is used to emulate the first and second level trigger. Finally, the simulated events are processed with the same code used for real data [92]. The beam and detector conditions are also taken into account in the simulation. This is specially important when considering the effects of multiple interactions. This run dependent $\mathrm{MC}$ tries to reproduce data by retrieving the status of the different systems from the detec-

tor data base and overlaying multiple interactions according to the instantaneous luminosity.

The hard scattering processes were generated using PYTHIA. For SM background estimation, the CDF validated version was used, Pyтнia 6.216. For signal $\phi \rightarrow \tau \tau$ Monte Carlo, however, a newer version was used, a customized release PүтніA 6.409. PyтнiA generator uses a leading order matrix element calculation for the hard scattering, with a tuned parton showering for radiation effects.

Pseudoscalar MSSM Higgs boson production via inclusive quark annihilation, $\mathrm{b} \overline{\mathrm{b}} \rightarrow \mathrm{A}^{0}$, process 157 , shows a softer spectrum than the exclusive channel $\mathrm{gg} \rightarrow \mathrm{A}^{0}+$ $\mathrm{b}$, process 189 . This was also the case for the outgoing b-quark $p_{\mathrm{T}}$ in inclusive $\mathrm{b} \overline{\mathrm{b}} \rightarrow \mathrm{Z}$ (Pythia process 1 ) and the exclusive channel $\mathrm{gg} \rightarrow \mathrm{Z}+\mathrm{b}$ (PYthia process 31), Figure 4.1. This issue was fixed after Рүтніa 6.4 for the neutral electroweak 
boson and a few releases later for the MSSM Higgs bosons.

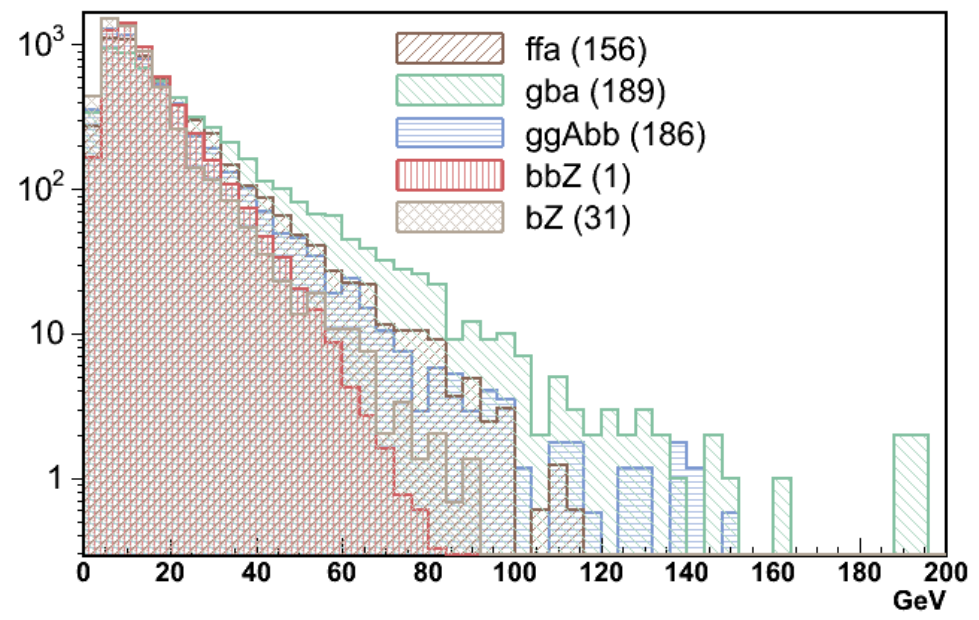

Figure 4.1: Comparison of the highest- $p_{\mathrm{T}}$ b-quark produced in the hard scattering in different processes in Pyтнia 6.216. The inclusive processes, 1 and 157, show a softer spectrum than the exclusive counterparts, 31 and 189

Both tree level processes, $g g \rightarrow \mathrm{A}^{0}$ and $\mathrm{b} \overline{\mathrm{b}} \rightarrow \mathrm{A}^{0}$, were generated separately and weighted accordingly. Given the expected sensitivity of this search, the samples were generated setting $\tan \beta=50$ and 14 mass points sweeping the range $90<\mathrm{m}_{\mathrm{A}^{0}}<250 \mathrm{GeV}$. The other sPythiA parameters are set to the Snowmass E3 (LC) group benchmark, parameter set 2, for heavy sparticles.

These samples were run-dependent generated, thus taking into consideration the detector conditions and overlying minimum bias. Electroweak and $\mathrm{t} \overline{\mathrm{t}}$ backgrounds were estimated from MC samples, also generated with PyтніA. All of these samples used leading order set of parton distribution functions, PDF, CTEQ5L [11]. Tau lepton are set to be treated as stable particles by PутнIA, because the decays are better simulated with TAUOLA [93]. 


\section{Chapter 5}

\section{Trigger efficiency}

\subsection{Introduction}

This chapter presents a measurement of the trigger efficiency of the track leg of the "lepton plus track" trigger paths. We measured the efficiency of these trigger paths with respect to fully reconstructed $\tau$ s using jets and di-lepton samples. We defined relevant variables to account for inefficiencies of the XFT track matching, L2 cluster matching and L3 isolated track finding. The overall trigger efficiency is the product of those three components.

\subsection{Trigger paths description}

The "lepton plus track" dataset contains events from several different trigger paths. Three families of those paths are relevant for this analysis. Two of them require a $\mu$ and an isolated track, "CMUP plus track" and "CMX plus track". A third family triggers on a central electron and an isolated track, "Electron plus track". The paths involving $\mu$ s were "dynamically prescaled" or "luminosity enabled" in the last periods of data taking. The whole list of trigger paths studied can be found in table 5.1 . 


\begin{tabular}{l|c}
\hline \hline Trigger Path Family & Trigger Paths involved \\
\hline "Electron plus track" & TAU_ELECTRON8_TRACK5_ISO \\
\hline "CMUP plus track" & TAU_CMUP8_TRACK5_ISO, tag 1 to 9 \\
& TAU_CMUP8_TRACK5_ISO_L2_LOOSE_DPS \\
& TAU_CMUP8_TRACK5_ISO_L2_LOOSE_LUMI_240 \\
& TAU_CMUP8_TRACK5_ISO_L2_LOOSE_LUMI_260 \\
\hline "CMX plus track" & TAU_CMX8_TRACK5_ISO \\
& TAU_CMX8_TRACK5_ISO_LUMI_200 \\
& TAU_CMX8_TRACK5_ISO_L2_LOOSE_DPS \\
\hline \hline
\end{tabular}

Table 5.1: "lepton plus track" trigger paths used in this analysis

Different versions of these trigger paths had different requirements. Only a few transitions affected the track leg of the trigger. The "Electron plus track" triggers underwent a transition after run 209770, when the isolation requirements at level 3 were modified and a level 2 calorimeter cluster was required to match the XFT track. The "CMUP plus track" and "CMX plus track" trigger paths also suffered two relevant changes. First, a level 2 XFT track was included simultaneously for both $\mu$ families, in the transition from tag 7 to 8 of the TAU_CMUP8_TRACK5_ISO path and from tag 6 to 7 of TAU_CMX8_TRACK5_ISO. Moreover, a second transition modified the level 3 isolation requirements after run 209770, simultaneous to the transition in the "Electron plus track".

\subsubsection{Level 2 trigger requirements}

An XFT track with a $p_{T}>5 \mathrm{GeV}$ is required in all the different versions of the TAU_ELECTRON8_TRACK5_ISO trigger paths. It is also required from tag 8 of the path TAU_CMUP8_TRACK5_ISO and tag 7 of TAU_CMX8_TRACK5_ISO.

Moreover, this XFT track has to match a cluster for the path TAU_ELECTRON8_TRACK5_ISO, versions 8 and 10. This cluster has to fulfill the requirements summarized in table 5.2 . 


\begin{tabular}{l}
$\overline{\frac{\text { L2 cluster requirements }}{E_{T}>4 G e V}}$ \\
Number of towers $\leq 5$ \\
pass $=2$ \\
\hline \hline
\end{tabular}

Table 5.2: L2 cluster cuts.

\begin{tabular}{c}
$\overline{\frac{\text { L3 track requirements }}{p_{T}>5 G e V}}$ \\
$|\eta|<1.5$ \\
isolation \\
\hline \hline
\end{tabular}

Table 5.3: Level 3 track cuts.

\subsubsection{Level 3 trigger requirements}

The level 3 trigger requirements are summarized in table 5.3. The isolation requirement is fulfilled when no shoulder tracks with sufficient $p_{T}$ and close enough in $Z$ lay in the isolation annulus. The definition of the isolation annulus and the cuts applied to select shoulder tracks was modified after run 209770. We refer to the trigger settings before this run as the "old trigger" and to the ones after that change as "new trigger". Table 5.4 shows the definition of isolation for both triggers.

\begin{tabular}{c|c|c}
\hline \hline & "old trigger" & "new trigger" \\
\hline L3 isolation annulus & $0.175<\Delta R<0.524$ & 10degrees $<$ Angle $<$ 30degrees \\
& $|\Delta Z|<15 \mathrm{~cm}$ & $|\Delta Z|<5 \mathrm{~cm}$ \\
track cuts & $p_{T}>1.5 \mathrm{GeV}$ & $p_{T}>1.5 \mathrm{GeV}$ \\
\hline \hline
\end{tabular}

Table 5.4: Isolation annulus definition, and cuts for shoulder tracks 


\subsection{Data Samples and event selection}

For this trigger study, we chose to use jet samples because they do not include track related requirements in their trigger paths. Therefore, these events are not correlated with the "lepton plus track" sample. This dataset, however, has the inconvenience of being populated mainly by fake $\tau$ s. This introduces a bias in the study of trigger level isolation, due to the differences in track multiplicity between jets and $\tau$ s. Moreover, a measurement of the efficiency of the level 2 calorimeter cluster requirement added to the "electron plus track" trigger path would be biased if measured in the jet samples.

We selected tight $\tau \mathrm{S}$ in the runs marked as good by the "good run list, em mu" version 13. Note that tight $\tau$ ID includes offline replication of level 3 isolation. After selecting $\tau \mathrm{s}$, we checked whether those $\tau \mathrm{s}$ would have passed the trigger requirements of the track leg of the different "lepton plus track" paths.

We also used high $p_{T}$ samples of both $\mu$ s and electrons for obtaining the absolute normalization of the level 3 efficiencies. We selected events in the "good run list, em mu", version 13, that had two almost-tight electrons or two almost-tight $\mu \mathrm{s}$ with an invariant mass consistent with the $\mathrm{Z}$ mass peak. By almost-tight we mean that we applied all the standard ID cuts except isolation. For the tracks of each of the leptons, we calculated the isolation related variables we use for $\tau \mathrm{s}$. We applied the same isolation cuts we apply to $\tau \mathrm{s}$ and then check whether these tracks would have passed the requirements in the "lepton plus track" paths.

\subsection{Level 2 trigger efficiency}

As previously stated, there are two kinds of requirements at level 2 that could potentially be applied in the different paths: XFT track matching and level 2 cluster matching.

We found the main source of XFT track finding inefficiency was related to the tracks crossing the central plane of the COT at the radius where the axial superlayers are located. Therefore, when the tracks cross the $z=0$ plane, we calculated

and parametrized the efficiency in terms of the radius of crossing of the central 


\begin{tabular}{c|r|r}
\hline \hline parameter & old trigger & new trigger \\
\hline$\epsilon_{\text {plateau }}$ & 0.9548 & 0.9539 \\
$A_{1}$ & 0.2646 & 0.2563 \\
$C_{1}$ & 58.92 & 58.91 \\
$S_{1}$ & 2.3810 & 2.3504 \\
$A_{2}$ & 0.3468 & 0.3320 \\
$C_{2}$ & 82.17 & 82.23 \\
$S_{2}$ & 2.6352 & 2.624 \\
$A_{3}$ & 0.4823 & 0.4604 \\
$C_{3}$ & 105.8 & 105.8 \\
$S_{3}$ & 2.796 & 2.812 \\
$A_{4}$ & 0.04457 & 0.03854 \\
$C_{4}$ & 131.0 & 130.8 \\
$S_{4}$ & 3.489 & 3.695 \\
\hline \hline
\end{tabular}

Table 5.5: Fitted parameters for the L2 trigger efficiency as a function of $R Z 0$.

plane of the COT, RZ0. When the tracks do not cross the central plane, then we found that the efficiency increases with the length of the track inside the COT.

The $R Z 0$ efficiency, figure 5.1, shows a very well defined behavior: a plateau of very high efficiency with four dips. We fitted the distribution to a flat plateau and 4 Gaussians:

$$
\epsilon(x)=\epsilon_{\text {plateau }}-\sum_{i=1}^{4} A_{i} e^{-\frac{\left(\frac{x-C_{i}}{S_{i}}\right)^{2}}{2}} .
$$

The fit shows the dips are precisely located at the radius where the axial superlayers of the COT are placed, table 5.5.

For the tracks that don't cross the $Z=0$ plane, we used the path length of the track in the $r-z$ plane inside the COT, Lrz. The longer this path is, the higher the efficiency, figure 5.2. Table 5.6 shows the result of the fit to a turn-on curve: 

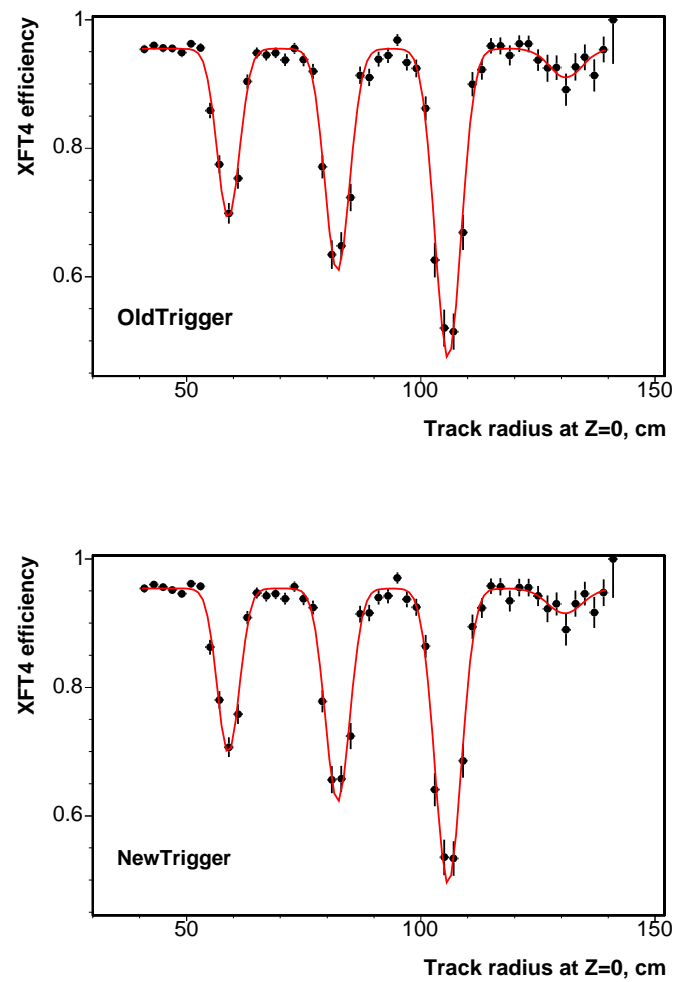

Figure 5.1: XFT track finding efficiency for tracks that cross the $Z=0$ plane. We parameterized this efficiency as a function of the radius at which the tracks cross that plane, $R Z 0$. 


\begin{tabular}{c|r|r}
\hline \hline parameter & old trigger & new trigger \\
\hline$\epsilon_{\text {plateau }}$ & 0.9750 & 0.9665 \\
$C$ & 1.958 & 24.63 \\
$S$ & 30.32 & 22.09 \\
\hline \hline
\end{tabular}

Table 5.6: Fitted parameters for the L2 trigger efficiency as a function of $\operatorname{Lr} z$.

$$
\epsilon(x)=\frac{\epsilon_{\text {plateau }}}{1+e^{\frac{C-x}{S}}}
$$

Regarding the cluster requirement, there is no dataset that would allow an independent measurement of the efficiency of a reconstructed $\tau$ to match a level 2 cluster with sufficient statistics. After several studies, we concluded that the only feasible way to approach this was relaying on Monte Carlo samples, like $Z \rightarrow \tau \tau$. Given that the simulation of the trigger, trigSim, is not fully consistent with data, we would have to scale our measurement of the trigger efficiency with a set of factors that can be obtained by from QCD Monte Carlo samples and the Jet samples, figure 5.3.

\subsection{Level 3 trigger efficiency}

The level 3 trigger requires a COT isolated track. As previously stated, there are two different implementations of isolation in our current datasets, corresponding to different time periods. We refer to them as "old trigger" and "new trigger". The cuts applied for these two definitions of isolation are summarized in table 5.4. Some of the $\tau$ ID cuts we apply offline are tighter that the ones applied at L3, like $p_{T}$ or $\eta$. In our range of acceptance, the trigger efficiency doesn't show a significant dependence on these variables.

However, L3 isolation is closely related to the goodness of the determination of the track parameters at trigger level. In the case when there are extra tracks in 

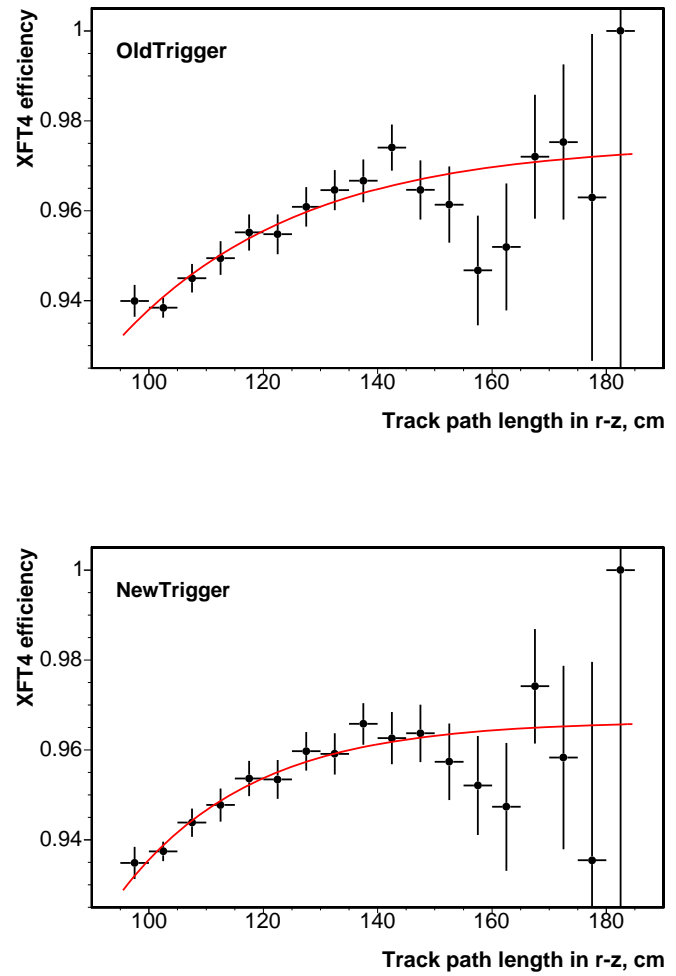

Figure 5.2: XFT track finding efficiency for tracks that don't cross the $Z=0$ plane. We parameterized this efficiency as a function of the length of the track path in the $r-z$ plane. 

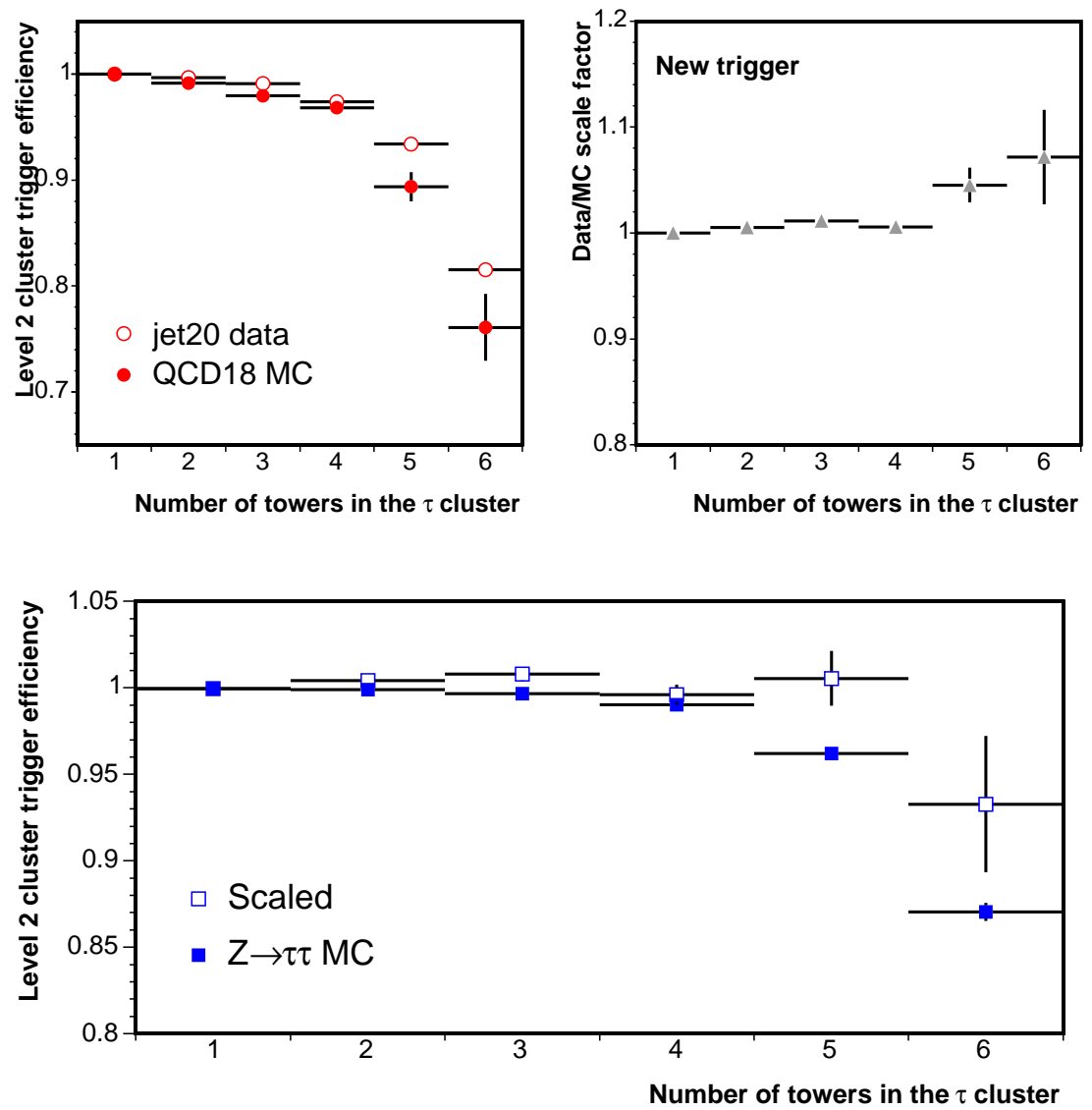

Figure 5.3: We compared the efficiency in Jet20 data and QCD $18 \mathrm{MC}$ in order to obtain a scale factor than we then applied to $Z \rightarrow \tau \tau$ MC. 
the signal cone, the variation of the parameters of these extra tracks could make them migrate from the signal cone at offline production level to the isolation cone at trigger level, leading to trigger inefficiency. Some parameters of the track like $\phi$ or the curvature, i.e. $p_{T}$, are determined much more precisely at trigger level than others, like $\cot \theta$. As it is shown in Figure 5.6 for instance, the trigger efficiency is related to the closeness of the extra tracks in the signal cone to the boundary of the cone. Moreover, the presence of tracks due to multiple interactions also has a dramatic impact on the efficiency of this trigger.

We selected events with 2 almost-tight electrons or two almost-tight $\mu$ s in the high-Pt samples. By almost-tight we mean we applied all standard cuts, except isolation. Then for each of the leptons in this events, we defined a 10degree cone, an got $\tau$-like signal and isolation variables: number of tracks in the signal cone, number of tracks in the isolation cone, sum of the $p_{T}$ of the tracks in the isolation cone, sum of the $E_{T}$ of $\pi^{0}$ in the isolation cone, .... We measured the trigger efficiency for the tracks of those leptons after applying $\tau$-like isolation cuts as a function of the number of primary verteces. This environment is closer to our signal, but it only compares to the jets samples in the 1-prong high- $p_{T}$ region. This measurement of the efficiency takes into account tracks that could lay in the isolation annulus, both tracks from other interaction, from the underlying event or fake tracks.

We account for track migration as a source of inefficiency by looking at the closeness of the extra tracks in the $\tau$ signal cone to the boundary. For the "old trigger", we calculated the $\Delta \theta$ of each extra track to the L3 signal cone boundary. We parameterized the efficiency as a function of smallest $\Delta \theta$ among those tracks, $\Delta \theta_{\text {min }}$. For the new trigger, the cone is defined in angle, instead of $\Delta R$. The natural variable to parameterize the efficiency in this case is, then, the angle to the cone cone boundary of the closest track to the boundary in the cone, $\Delta \alpha_{\min }$. For these measurement we ran over the events on the jet samples. However, calculating the efficiency in the jet samples could introduce a bias in our measurement because of the differences in track multiplicity between jets and $\tau \mathrm{s}$. Most of the $\tau \mathrm{s}$ we reconstruct are fakes, which are more likely to fail trigger requirements because of the presence of low $p_{T}$ tracks in the trigger isolation cone. In order to avoid this bias, and to make this "track migration" efficiency multiplicative with the overall efficiency we obtained from the jet samples, we only used $\tau$ candidates that were 


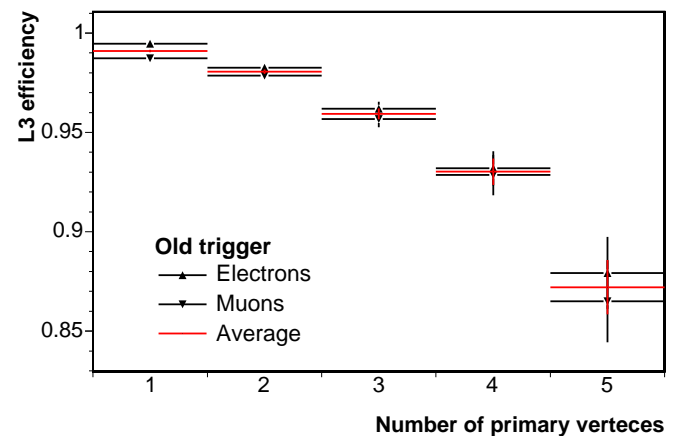

(a) No L2 requirements

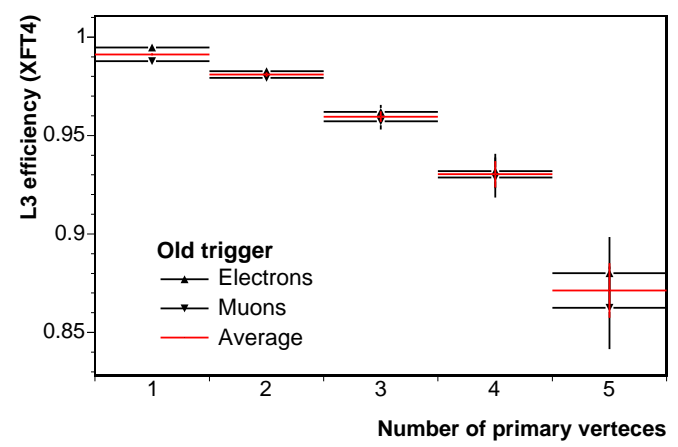

(b) XFT track matched

Figure 5.4: L3 efficiency parameterized in terms of the number of primary verteces for the old trigger. 


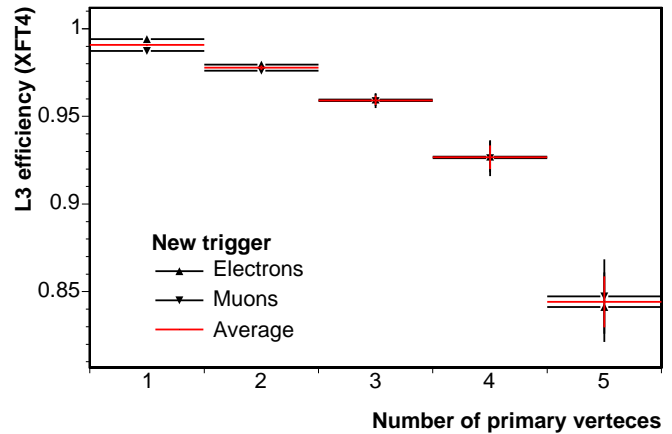

(a) XFT track matched

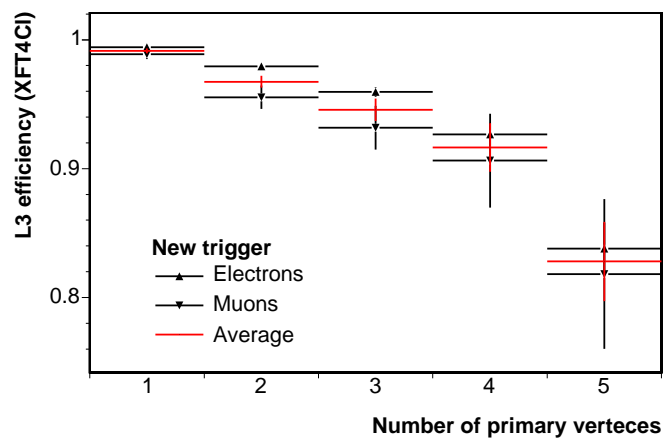

(b) XFT track and L2Cluster matched

Figure 5.5: L3 efficiency parameterized in terms of the number of primary verteces for the new trigger. 


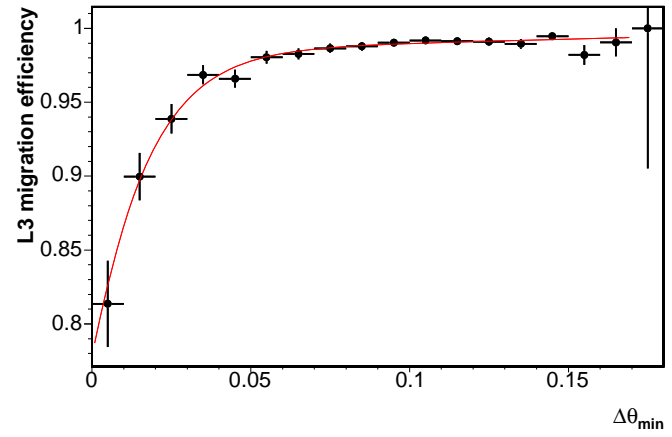

(a) No L2 requirements

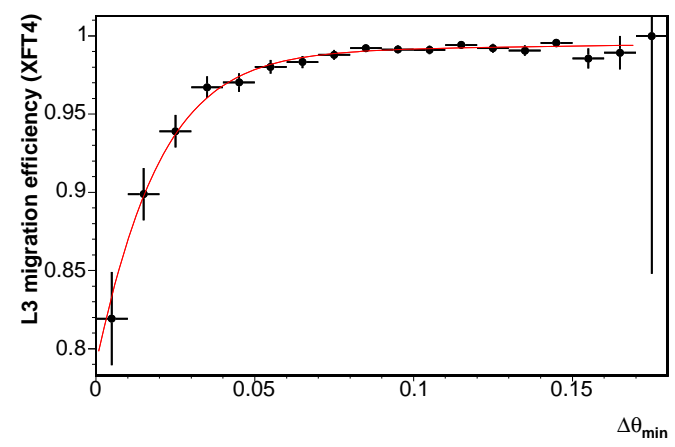

(b) XFT track matched

Figure 5.6: L3 "migration" efficiency for the "old trigger", parameterized in terms of $\Delta \theta_{\min }$ 


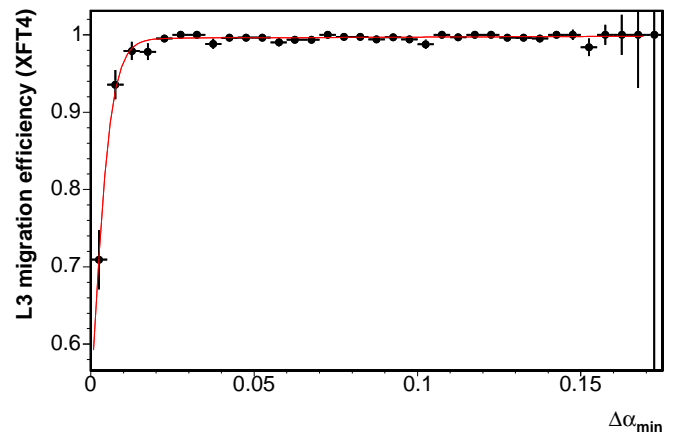

(a) XFT track matched

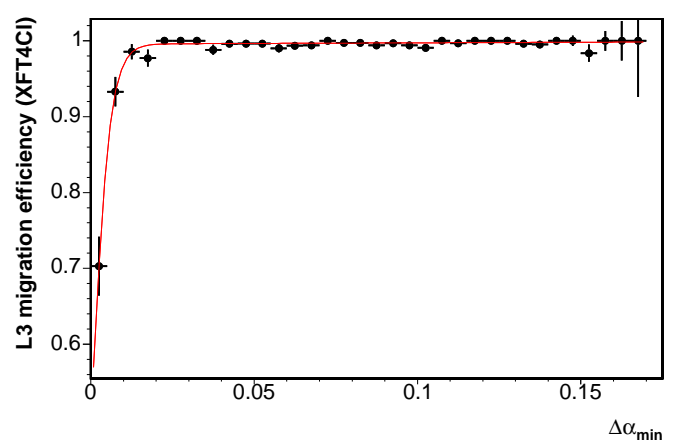

(b) XFT track and L2Cluster matched

Figure 5.7: L3 "migration" efficiency for the "new trigger", parameterized in terms of $\Delta \alpha_{\min }$ 


\begin{tabular}{c|rr|rr}
\hline \hline \multirow{2}{*}{ parameter } & \multicolumn{2}{|c|}{ "old trigger" } & \multicolumn{2}{c}{ "new trigger" } \\
& no L2 & XFT & XFT & XFT+L2Cluster \\
\hline$\epsilon_{\text {plateau }}$ & 0.9848 & 0.9896 & 0.9955 & 0.9958 \\
$\epsilon_{\text {slope }}$ & $-2.011 \times 10^{-2}$ & $-2.312 \times 10^{-2}$ & $-2.991 \times 10^{-4}$ & $3.9491 \times 10^{-5}$ \\
$C$ & $1.517 \times 10^{-2}$ & $1.678 \times 10^{-2}$ & $2.985 \times 10^{-3}$ & $2.7921 \times 10^{-3}$ \\
$S$ & $5.272 \times 10^{-2}$ & $2.543 \times 10^{-2}$ & $1.598 \times 10^{-2}$ & $1.5801 \times 10^{-2}$ \\
\hline \hline
\end{tabular}

Table 5.7: Results of the fits to $\Delta \theta_{\min }$ and $\Delta \alpha_{\text {min }}$.

surrounded by a very clean environment. This condition was achieved by requiring no extra tracks in a cone of $\Delta R=0.7$

Both the overall efficiency and the track migration need to be calculated for each different set of L2 and L3 requirements. Figure 5.4 shows the L3 efficiency for the "old trigger" and Figure 5.5 for the "new trigger", considering the different L2 requirements in each trigger path.

Figure 5.6 and Figure 5.7 show the "L3 migration" efficiency in terms of $\Delta \theta_{\text {min }}$ and $\Delta \alpha_{\min }$, fitted to a turn-on function:

$$
\epsilon(x)=\frac{\epsilon_{\text {plateau }}+\epsilon_{\text {slope }} \times x}{1+e^{\frac{C-x}{S}}}
$$

The results of the fits are shown in table 5.7. 


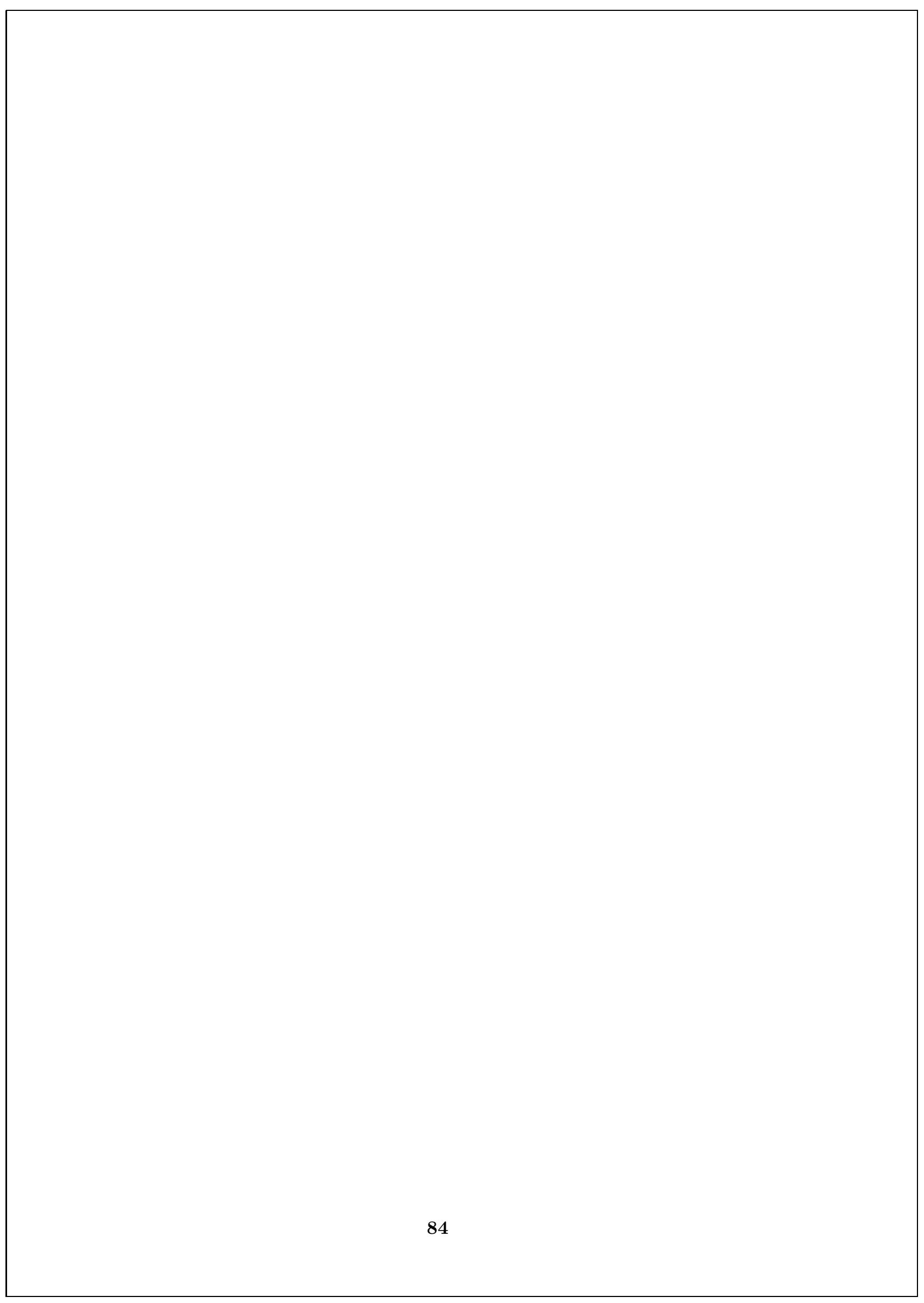




\section{Chapter 6}

\section{Particle identification}

The particles of interest in this analysis are electrons (from $\tau_{e}$ ), muons (from $\tau_{\mu}$ ), and the products of hadronically decaying taus. The selection criteria for electrons and muons closely follows the usual CDF recommendations. Our minimum $p_{\mathrm{T}} / E_{\mathrm{T}}$ requirements for muons and electrons are set to $6 \mathrm{GeV}$ for the $\tau_{e} \tau_{\mu}$ channel, and to $10 \mathrm{GeV}$ for the $\tau_{h a d} \tau_{e / \mu}$ channels.

We apply the standard cuts for tight medium- and high- $p_{T}$ leptons (excluding isolation), and use the common CDF reconstruction and identification DATA/MC scale factors. The results for electrons and muons can be found in [94] and [95]. Muon reconstruction efficiencies are consistent in the high and medium- $p_{T}$ samples, and we use the data/MC scale factors as quoted in CDF official documentation [96].

For the electron and muon selection in the $\tau_{e} \tau_{\mu}$ channel we do not apply calorimeter isolation and use a fixed track isolation cuts instead: $I_{t r k}^{0.4}=\sum p_{T}^{i s o}<2 \mathrm{GeV}$, where the sum is over all tracks within $\Delta R<0.4$ and $\Delta z_{0}<5 \mathrm{~cm}$ of the muon or electron track. For the $\tau_{l} \tau_{\text {had }}$ channels, we additionally apply the calorimeter isolation as defined for low/medium- $p_{T}$ leptons $[94,95]$. 


\subsection{Electrons}

The following is a list of the electron ID cuts used in the analysis:

- $E_{T}>10.0 \mathrm{GeV}$ for $\tau_{l} \tau_{\text {had }}, E_{T}>6.0 \mathrm{GeV}$ for $\tau_{e} \tau_{\mu}$

- $p_{T}>8.0 \mathrm{GeV}$ for $\tau_{l} \tau_{h a d}, p_{T}>5.0 \mathrm{GeV}$ for $\tau_{e} \tau_{\mu}$

- $E_{\text {had }} / E_{E M}<0.055+0.00045 * E$

- $L_{s h r}<0.2$

- $E / P<2.0$ (no cut if $E_{T}>50 \mathrm{GeV}$ )

- $-3<q \Delta X<1.5 \mathrm{~cm}$

- $|\Delta Z|<3 \mathrm{~cm}$

- $\chi_{C E S \text { strip }}^{2}<10$

- $\left|z_{0}\right|<60 \mathrm{~cm}$

- $\left|d_{0}^{\text {corr }}\right|<0.2 \mathrm{~cm}$

- $\left|z_{C O T}\right|<140.0 \mathrm{~cm}$

- $\geq 3$ stereo, 2 axial layers ( $\geq 5$ hits)

- $E_{\text {rel }}^{i s o}<0.1$ or $E_{T}^{i s o}<2.0 \mathrm{GeV}$, not applied for $\tau_{e} \tau_{\mu}$

- $\sum p_{T}^{i s o}<2 \mathrm{GeV}($ cone 0.4$)$

- satisfy fiduciality requirements

- not from conversion

Conversion removal is applied to electron candidates by forming all possible oppositely charged track pairs and rejecting the candidate if at least one satisfies $\left|S_{X Y}\right|<0.2 \mathrm{~cm},|\Delta \lambda|<0.04$.

The data/MC scale factor of the fixed track isolation cut is determined from $e e$ in the $Z$ mass peak. We use data from the SUSY dilepton ee sample and $Z / \gamma \rightarrow e e$ 

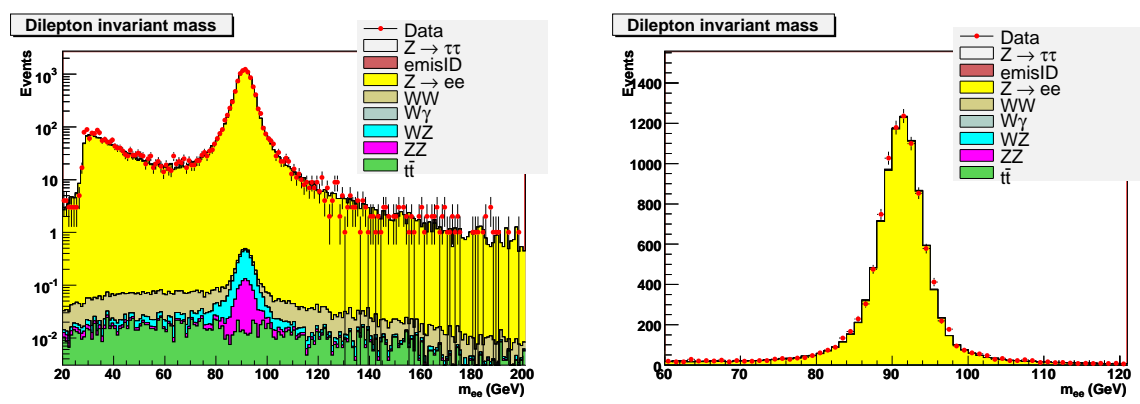

Figure 6.1: Invariant mass of the selected electron pairs ( $1 \mathrm{fb}-1$ sample).

oficial MC samples. The electrons are required to pass the full electron ID except for isolation. We find that the MC describes the data quite well and obtain a data/MC scale factor of $0.997 \pm 0.001$ for the track isolation cut.

For this type of isolation there should be no dependence on electron $E_{\mathrm{T}}$ as illustrated on Figure 6.2 (shown later in the text), where we plot the scale factor as a function of electron $E_{T}$. To reduce backgrounds in the low- and intermediate- $E_{T}$ region, we select only back-to-back electron pairs $(\Delta \phi>3.0)$. We would like to note that this distribution is for demonstration purposes to show that there is no general $E_{T}$-trend. The $E_{T}<15 \mathrm{GeV}$ region is probably more sensitive to the background subtraction rather than the efficiency ratio itself. The other issue regarding electrons is the data/MC energy scale. The plots on Figure 6.1 show the invariant mass of lepton pairs and the data/MC agreement in the position of the $Z$-peak (after applying all the recommended corrections in CDF).

\subsection{Muons}

The analysis uses central muons reconstructed in the CMU and CMP and CMX detectors, with the following cuts

- $p_{T}>10.0 \mathrm{GeV}$ for $\tau_{l} \tau_{h a d}, p_{T}>6.0 \mathrm{GeV}$ for $\tau_{e} \tau_{\mu}$

- $\left|z_{0}\right|<60 \mathrm{~cm}$ 
- $\left|d_{0}^{\text {corr }}\right|<0.2 \mathrm{~cm}$

- $\sum p_{T}^{i s o}<2 \mathrm{GeV}($ cone 0.4$)$

- $E_{\text {rel }}^{i s o}<0.1$ or $E_{T}^{i s o}<2.0 \mathrm{GeV}$, not applied for $\tau_{e} \tau_{\mu}$

- $\geq 3$ stereo, 2 axial layers ( $\geq 5$ hits)

- $\rho_{C O T}>140 \mathrm{~cm}$

$\underline{p_{T}>20 \mathrm{GeV}}$

- $E_{E M}<2+\max (0,0.0115 *(p-100)) \mathrm{GeV}$

- $E_{\text {had }}<6+\max (0,0.028 *(p-100)) \mathrm{GeV}$

- $\left|\Delta X_{C M U}\right|<3 \mathrm{~cm}$ and $\left|\Delta X_{C M P}\right|<7 \mathrm{~cm}(\mathrm{CMUP})$

- $\left|\Delta X_{C M X}\right|<6 \mathrm{~cm}(\mathrm{CMX})$

$\underline{p_{T}<20 \mathrm{GeV}}$

- $E_{E M}<2 \mathrm{GeV}$

- $E_{\text {had }}<3.5+\left(p_{T} / 8.0\right) \mathrm{GeV}$

- $\left|\Delta X_{C M U}\right|<3 \mathrm{~cm}$ or $\chi_{C M U}^{2}<9.0$

- $\left|\Delta X_{C M P}\right|<7 \mathrm{~cm}$ or $\chi_{C M P}^{2}<9.0$

- $\left|\Delta X_{C M X}\right|<6 \mathrm{~cm}$ or $\chi_{C M X}^{2}<9.0$

Just like in the electron case we measure the additional data/MC scale factor for the track isolation requirement from events in the $Z$-peak and get a data/MC scale factor of $1.00 \pm 0.001$.

As expected the scale factor has no $p_{T}$ dependence. The distribution, together with the results for electrons is shown in Figure 6.2. The di-muon mass distributions in the data and MC are in good agreement - Figure 6.3. 

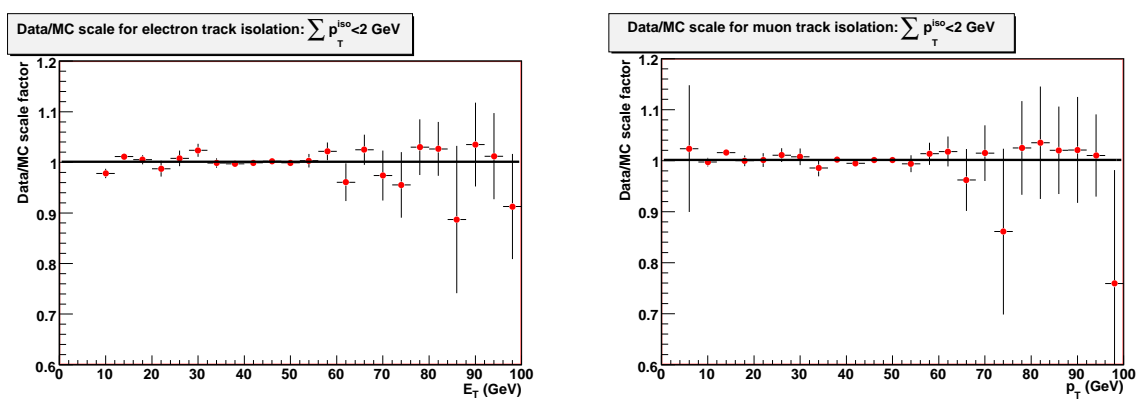

Figure 6.2: Data/MC scale factor for track isolation of electrons (left), and muons (right). The scales are plotted as a function muon $p_{T}$ (electron $E_{T}$ ).
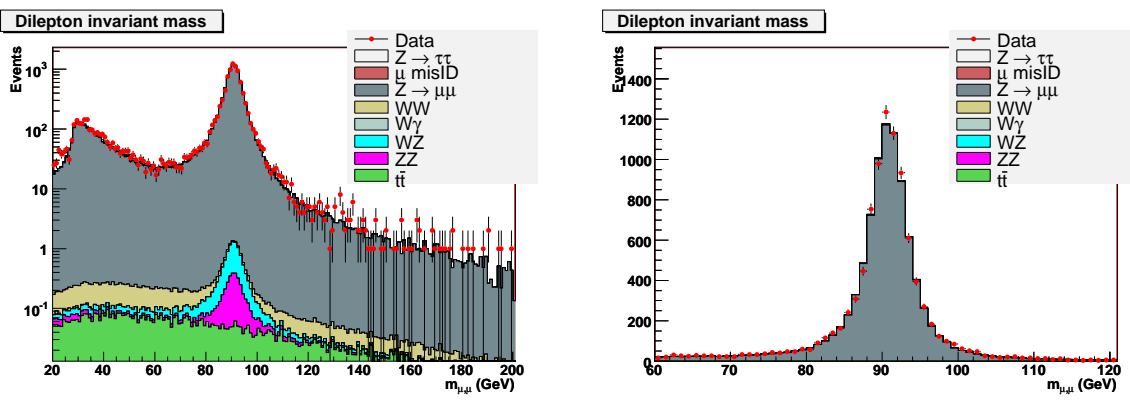

Figure 6.3: Invariant mass of selected muon pairs ( $1 \mathrm{fb}-1$ sample). 


\subsection{Taus}

Here and below "tau reconstruction" refers to the reconstruction of the visible decay products $X_{h}$ of taus experiencing semi-hadronic decays: $\tau \rightarrow X_{h} \nu_{\tau} . X_{h}$ can be a $\pi^{ \pm} / K^{ \pm}$, or some short-lived intermediate resonance that decays directly (or through some intermediate states) to final states containing $\pi^{ \pm, 0}, K^{ \pm, 0}$.

Details on the tau reconstruction procedure can be found in previous papers [97, 98] and the references therein. The selection criteria used in this analysis are similar, with the exception of the mass cut in the 3-prong taus,and the track isolation requirement. Here we only list the selection cuts as used in this analysis and the derived data/MC scale factors.

Tracks and $\pi^{0}$ 's in the signal cone are used to construct the four-momentum of the hadronic system. The four-momentum is used in subsequent event cuts and for the determination of the mass of the system $M\left(\operatorname{trks}+\pi^{0} s\right)$. In some cases there is a non-negligible energy loss due to $\pi^{0}$ reconstruction inefficiency (mostly when they hit near the edges of the CES detectors). Therefore, in some cases one has to apply corrections to the measured tau energy from tracks and $\pi^{0}$ 's, as discussed in [99]. Here we apply similar (but simplified) corrections.

Corrections are mostly needed for the 1-prong tau decays, and we correct the energies only in this case using information on the energy deposited in the tau calorimeter cluster. We use two corrections that are applied only in cases when the energy in the calorimeter cluster is larger than the estimate from tracks $+\pi^{0}$ 's. First, we want to account for the case with possible $\pi^{0}$ losses. This correction is applied when we have substantial EM energy in the cluster $\left(E_{E M} / E_{t o t}>0.2\right)$. In this case the we assign as tau $p_{T}$ the sum of the energy of the charged track and the EM energy contained in the cluster (minus MIP energy). To avoid overestimation due to large EM energy deposition from the track, we require that $E_{\text {had }}>0.3 p^{t r k}$. In cases where the conditions for this correction are not met we look for cases of potentially large hadronic energy contribution (possibly due to $K_{L}$ ): $p^{\text {trk }}<$ $E_{\text {had }}-\sigma_{\text {had }}$, where $\sigma_{\text {had }}=0.5 \sqrt{E_{\text {had }}}$. In this case we assign $E_{T}^{\tau c l}$ as tau $p_{T}$ instead of $p_{T}\left(\operatorname{trks}+\pi^{0} s\right)$.

We define the variable $\xi^{\prime}$ to suppress electrons and muons depositing large amount 
of EM energy.

$$
\xi^{\prime}=\frac{E_{t o t}}{\sum|\vec{p}|}\left(0.95-\frac{E_{E M}}{E_{t o t}}\right)
$$

where $E_{t o t}, E_{E M}, E_{h a d}$ are the total, electromagnetic and hadronic tau cluster energies, and $\vec{p}$ are the momenta of charged tracks associated with the tau.

To suppress electrons accompanied by bremsstrahlung, we reject 1-prong tau candidates if a $\pi^{0}$ candidate with $\left|\Delta z_{C E S}\right|<2.0 \mathrm{~cm}$ of the projection of the track and in $\phi$ lies between the CES intersect of the track helix and its tangential. To account for CES position, and track extrapolation resolution, the veto region is extended by $0.01 \mathrm{rad}$ beyond the points of the tangential intersect track hit in CES. This procedure is similar to the one used in before [74]. In the previous analysis we also suppressed electrons not accompanied by bremsstrahlung using information from the CES clusters matched to the track. It is a very powerful electron removal tool, but it is not used in this iteration of the analysis. It will be reintroduced after some additional studies are performed on CES calibration and data/MC removal efficiency. The track in one-prong taus in the $\tau_{e} \tau_{\text {had }}$ channels are restricted to the fiducial part of the CES detector $\left|x_{C E S}\right|<21 \mathrm{~cm}$. This restriction is imposed to avoid the effect of deficiencies in the simulation of detector response to electrons near the $\phi$ cracks.

The following is a summary of the tau reconstruction and ID cuts.

- $E_{T}^{\text {seed twr }}>6.0 \mathrm{GeV}$

- $E_{T}^{s h t w r}>1.0 \mathrm{GeV}$

- $N^{t w r} \leq 6$

- $\theta_{\text {sig }}=\min \left(0.17, \frac{5.0 \mathrm{rad} / \mathrm{GeV}}{E^{\tau c l}}\right) \mathrm{rad} \quad 1$

- $\theta_{\text {iso }}=0.52 \mathrm{rad}$

\footnotetext{
${ }^{1}$ To prevent the signal cone from becoming too small and sensitive to track $/ \pi^{0}$ direction resolution we set limits $0.05<\theta_{\text {sig }}$ rad for tracks, and $0.1<\theta_{\text {sig }} \operatorname{rad}$ for $\pi^{0}$ 's
} 
- $p_{T}^{\text {seed }}{ }^{\text {trk }}>6.0 \mathrm{GeV} \quad 2$

- $p_{T}^{\text {sh }}{ }^{\text {trk }}>1.0 \mathrm{GeV}$

- $E_{T}^{\tau c l}>9.0 \mathrm{GeV}$

- $p_{T}>15.0 \mathrm{GeV}$ for 1-prongs, $p_{T}>20 \mathrm{GeV}$ for 3-prongs

- $\Delta z^{\text {sh trk }}<5.0 \mathrm{~cm}$

- $9.0<\mid z_{C E S}^{\text {seed }}$ trk $\mid<230.0 \mathrm{~cm}$

- traverse all 4 axial SL's in COT

- $\sum p_{T, t r k}^{i s o}<2 \mathrm{GeV}$, no tracks with $p_{T}>1.5 \mathrm{GeV}$

- $\sum E_{T, \pi^{0}}^{i s o}<1 \mathrm{GeV}$

- $N_{\text {sig }}^{\text {trk }}=1,3$

- $\left|\sum Q^{\operatorname{trk}}\right|=1$

- $M\left(\operatorname{trks}+\pi^{0} s\right)<1.8 \mathrm{GeV}$

- $\xi^{\prime}>0.1$

Tau detection efficiency is affected by the requirements imposed on tracks and $\pi^{0}$ 's in the isolation annulus. Possible difference between data and MC can appear due to deficiency in the simulation of the underlying event and multiple interactions. To compare data and MC we select muons and electrons from $Z \rightarrow \mu \mu$ and $Z \rightarrow e e$ events and compare the tau-style isolation efficiencies. In this case the isolation annulus is fixed to be between $10^{\circ}-30^{\circ}$ degrees with respect to the selected lepton. In principle this comparison can be done by randomly selecting regions of the detector, but the use of $\mathrm{Z}$ events ensures environment that is similar to our event selection.

The electrons (or muons) are required to have opposite charge, pass the standard tight ID cuts for high- $p_{T}$ leptons (up to isolation), and have an invariant mass $66<M_{l l}<116 \mathrm{GeV}$.

\footnotetext{
${ }^{2}$ Seed track quality: $\geq 3$ stereo, $\geq 2$ axial layers with at least 5 hits
} 
For the selected leptons we replicate the L3 isolation offline (just like for tau selection) and determine the data/MC scale factors and uncertainties for the isolation requirements $\sum p_{T, t r k}^{i s o}<2 \mathrm{GeV}, \sum E_{T, \pi^{0}}^{i s o}<1 \mathrm{GeV}$. The isolation efficiencies obtained from electrons and muons as a function of number of primary verteces are shown in Figure 6.4. One can clearly see the deterioration with increased number of interactions in the event. For practical purposes we derive one single scale factor and uncertainty by integrating over all vertex multiplicities. The average scale factor from electrons and muons is $\epsilon_{\text {data }}^{i s o} / \epsilon_{M C}^{i s o}=0.989 \pm 0.001$ (runs $<209769$ ), and $\epsilon_{\text {data }}^{i s o} / \epsilon_{M C}^{i s o}=0.984 \pm 0.001$ for (runs $>209769$ ).

We use the same procedure to determine the scale factor for the offline replication of the L3 isolation requirements, given that the tau isolation requirements are satisfied. We find $\epsilon_{\text {data }}^{L 3}$ iso $/ \epsilon_{M C}^{L 3}$ iso $=0.997 \pm 0.001$ for the period of the "old trigger" (runs $<209769$ ), and $\epsilon_{\text {data }}^{L 3 \text { iso }} / \epsilon_{M C}^{L 3}$ iso $=0.999 \pm 0.001$ (runs> 209769).

Tracks with $p_{T}>1.0 \mathrm{GeV}$ from the underlying event and multiple interactions can contribute to track "multiplicity migration" if they are contained in the signal cone. We compare the number of additional tracks in a $10^{\circ}$ cone with respect to the muon direction and find an average of $0.025 \pm 0.013$ additional tracks in the data, and $0.027 \pm 0.003$ in the MC. This corresponds to an uncertainty in tau selection efficiency due to "multiplicity migration" of $0.3 \%$.

Hadronic scale uncertainty in the MC affects tau acceptance through the requirement on minimum tau cluster energy, tau seed tower $E_{T}$ threshold, and the $\xi^{\prime}$ cut. In the previous iteration we found an uncertainty of $2 \%$, consistent with the result obtained in $\mathrm{CDF}$ [100].

The effect of the cut on the mass of the hadronic tau decay products $m\left(t r k s+\pi^{0}\right.$ 's) on data and MC is examined using a sample of taus from $\mathrm{W}$ decays. The data is selected with the "tau+MET" trigger. For this test we use the $0 \mathrm{~h}+0 \mathrm{i}$ samples (0d stntuples were not available at the time). Tau ID is the same as for the Higgs search, except for a higher threshold on the seed track: $p_{T}>10 \mathrm{GeV}$ due to the trigger requirements. To suppress multi-jet backgrounds we impose tight event cuts: $E_{\mathrm{T}}^{\mathrm{miss}}>30 \mathrm{GeV}$, no extra jets with $E_{T}>5 \mathrm{GeV}$ in the detector. The latter requirement introduces a large dependence on the modeling of jet multiplicities (including very soft jets), that complicates the exact determination of tau yields in data and MC. Since we are not attempting to measure the $W \rightarrow \tau \mu$ production, 


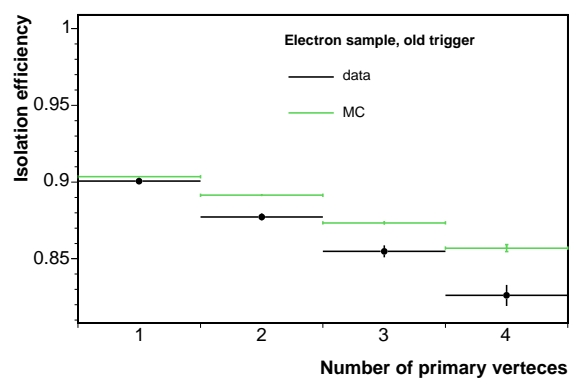

(a) Efficiency measured with $Z \rightarrow e e$ events, run $<209769$

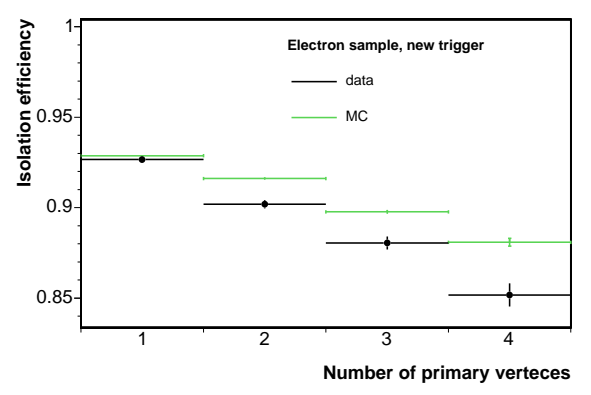

(c) Efficiency measured with $Z \rightarrow e e$ events, run > 209769

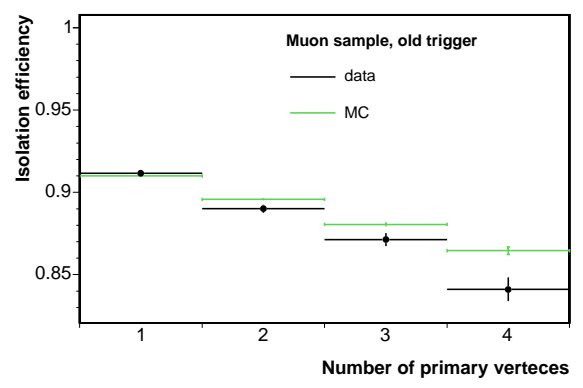

(b) Efficiency measured with $Z \rightarrow \mu \mu$ events, run $<209769$

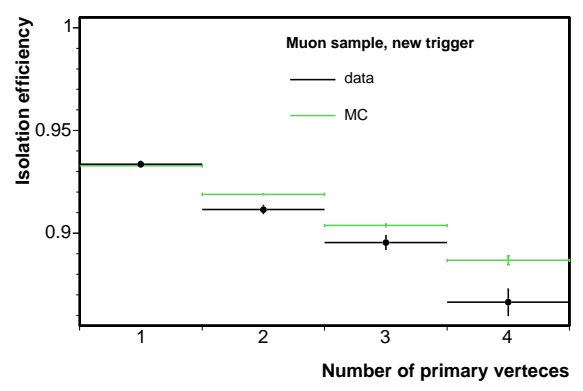

(d) Efficiency measured with $Z \rightarrow \mu \mu$ events, run $>209769$

Figure 6.4: Comparison of the efficiency of the $\sum p_{T, t r k}^{i s o}<2 \mathrm{GeV}, \sum E_{T, \pi^{0}}^{\text {iso }}<$ $1 \mathrm{GeV}$ cuts in data and MC using $Z \rightarrow e e, \mu \mu$ events $\left(1.8 \mathrm{fb}^{-1}\right.$ sample). 
we only need to determine the relative contributions from the major processes that contribute to the selected events. The relative contributions of the MC events from EW processes are fixed to the ratios of their cross sections. The number of residual $j e t \rightarrow \tau$ fakes is obtained by performing a fraction fit of the track multiplicity distribution with templates from MC and a JET20 sample. The fitted distribution is shown in Figure 6.5.

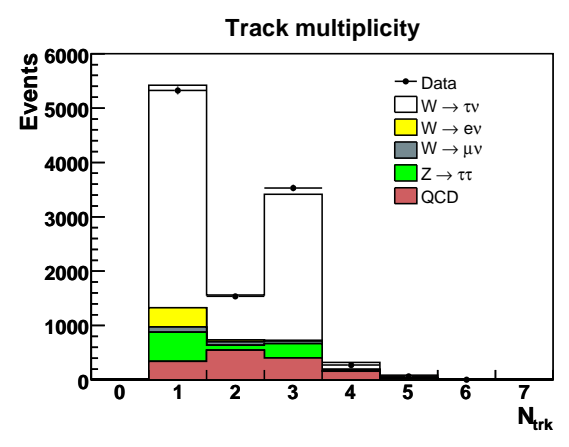

(a) Fitted track multiplicity distribution for tau candidates from the $\tau+E_{\mathrm{T}}^{\prime}$ sample

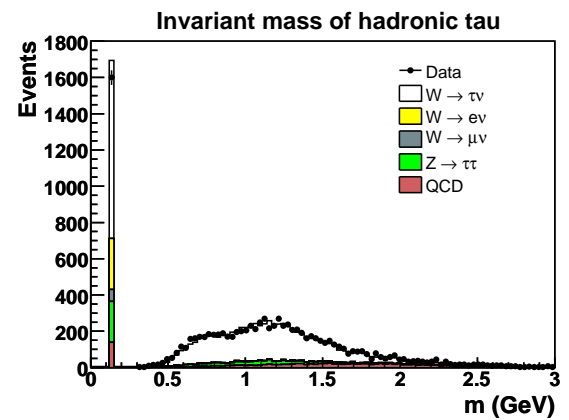

(b) Mass of the hadronic decay products (1,3-prong)

Figure 6.5: Track multiplicity and $m\left(t r k s+\pi^{0}\right.$ 's $)$ for tau candidates. Contributions from $W \rightarrow \tau \nu$ and various backgrounds are included as shown in the Legend.

The mass cut $(m<1.8 \mathrm{GeV}$ ) efficiency for 1-prongs is $0.952 \pm 0.003$ for data and $0.969 \pm 0.003$ in the MC. From here we determine $\epsilon_{\text {data }}^{m \text { cut }} / \epsilon_{M C}^{m}$ cut $=0.982 \pm 0.05$. To account for deficiencies in the reconstructed mass in the data we decided to relax the mass in the 3-prong samples to $2.2 \mathrm{GeV}$, and find efficiency of $0.944 \pm 0.004$ for data and $0.978 \pm 0.003$ in the MC, resulting in a data/MC scale factor of $\epsilon_{\text {data }}^{m \text { cut }} / \epsilon_{M C}^{m}$ cut $=0.965 \pm 0.005$. Due to the discrepancy in the mass distributions in the high-mass region for 3-prongs we increase the uncertainty on the mass cut efficiency to $2 \%$.

Based on the data/MC comparisons we assign a $3 \%$ systematic uncertainty on the selection of hadronically decaying taus. When applicable, the MC events are scaled using the derived scale factors. 
The determination of the tau energy scale is not straight forward. The presence of neutrinos in tau decays prevent us from making reconstructing narrow resonances like it is done for electrons and muons. Here we use a comparison of the data and MC $p_{T}$ distributions of reconstructed taus in $W \rightarrow \tau \nu$ decays and look for indication of relative data/MC energy shifts. In the previous iteration of the analysis [101] we derived corrections of the order of $1 \%$ to be applied to the data. We use the same procedure and confirm, that these corrections are still valid for the new sample as shown in Figure 6.6.

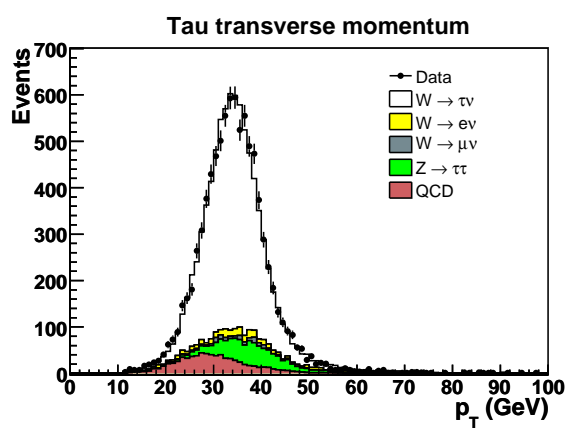

(a) $p_{T}$ spectrum of the decay products in hadronic tau decays.

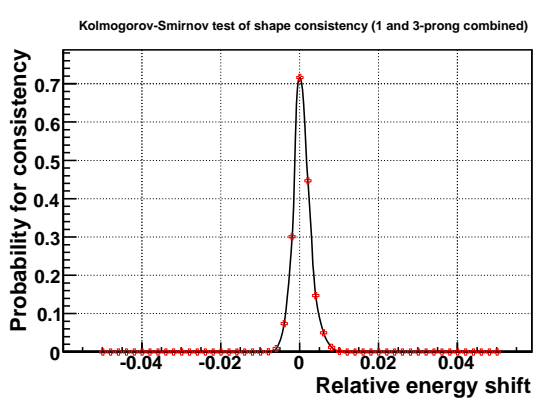

(b) $p_{T}$ spectrum of the decay products in hadronic tau decays.

Figure 6.6: Distributions of tau $p_{T}$ in data and MC after correcting data for the observed shifts (left), and the resulting KS test (right).

The $p_{T}$ distributions and the corresponding KS test results after applying the previously derived corrections are shown in Figures 6.6.

\subsection{Missing transverse energy}

The presence of $E_{\mathrm{T}}^{\mathrm{miss}}$ in an event (apart from the instrumental part) is an indication of the presence of "invisible" particle(s). In the studies topological states we have three or four neutrinos. Therefore, $E_{\mathrm{T}}^{\mathrm{miss}}$ represents the transverse component of the sum of the neutrinos momenta (and it is more appropriate to call it missing transverse momentum). Most of the events have taus that are almost 
back-to-back in the transverse plane and the neutrino momenta are balanced to a large degree. As a result the signal signature is not always characterized by a large $E_{\mathrm{T}}^{\text {miss }}$.

$E_{\mathrm{T}}^{\mathrm{miss}}$ is used both in the event selection cuts and signal extraction. The "raw" $E_{\mathrm{T}}^{\text {miss }}$ in the event is calculated for the $z$-position of the primary vertex (if no vertex is present, the $z$-position of the leptons is used). We apply muon corrections when a track passes the muon ID requirements, by replacing the transverse energy in the hit tower(s) by the track $p_{T}$. Similarly, the tau calorimeter cluster $E_{T}$ is replaced by the calculated tau $p_{T}$. Calorimeter response to jets is taken into account by applying the jet energy corrections up to (and including) Level 5 to all jets with $E_{T}^{\text {raw }}>10 \mathrm{GeV}$ in the region $|\eta|<2.4$ (if they are not identified as $e, \mu$, or $\tau$ ). 


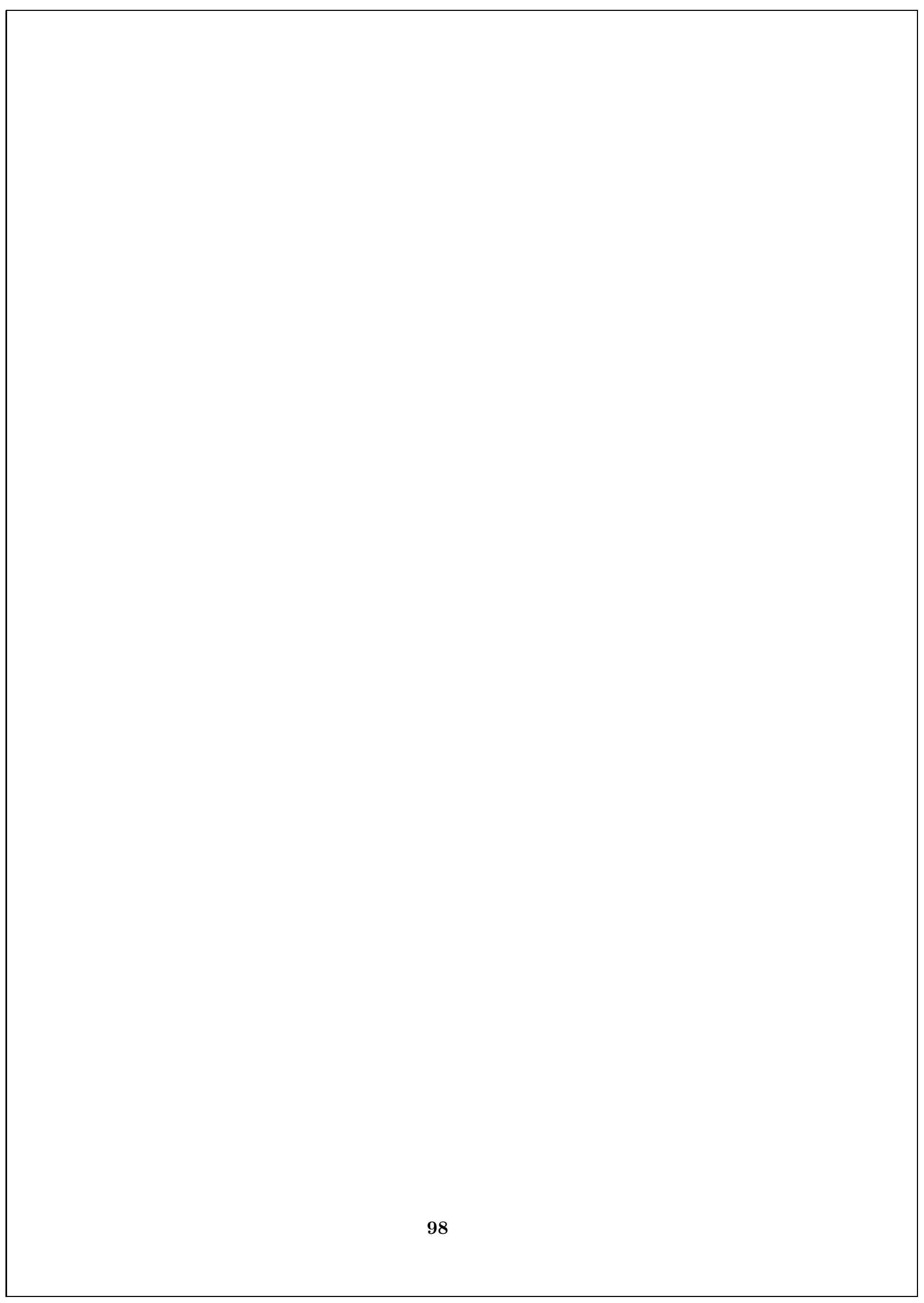




\section{Chapter 7}

\section{Event Selection and Background Estimation}

\subsection{Fake Rate Measurement and Consistency Checks}

Using ST5, JET 20, 50, 70, 100 samples we have determined the $j e t \rightarrow \tau$ fake rates to be used in QCD background estimation. We are interested in relative fake rates. We used parametrization in terms of $E_{T}^{c l}+E_{T}^{c a l}$ iso,$\eta_{\text {det }}$, and track multiplicity in the signal cone. Apart from the change in parametrization, the procedure for the fake rate determination is the same as the one described in previous publications [97]. Here we present the results obtained for the current set of tau identification cuts, and applicable to the larger data sample used in this analysis.

In the fake estimations we use the highest- and second-highest energy jets in the event. The reasoning behind this choice is that the $j e t \rightarrow \tau$ backgrounds in our sample are dominated by di-jet and multi-jet events. We take the average of the two estimates as central value and assign the half-difference as systematic uncertainty. There is no discrepancy between the fake rates obtained with the different samples. 
The next important step is to verify that these fake rates provide adequate description of the backgrounds expected to pass our final selection. The sources contributing to the $j e t \rightarrow \tau$ backgrounds were listed in Section 7.3.3. We identify three independent samples that allow testing of the fake rate predictions against the actual number of observed events. The source are examined separately to account for possible jet property differences.

- multi-jet sample passing event cuts:

Non-isolated leptons (or lepton fakes) selected by explicitly requiring tracks in the isolation cone around the lepton candidate $(e, \mu)$. Event cuts are applied without modification.

- $W+j e t(s)$ :

Select control region in the $P_{\zeta}$ vs $P_{\zeta}^{v i s}$ plane as indicated on Figure 7.7 . Obviously the $\zeta$ cut is replaced, however, this should have no effect on the jet properties. Because of the different kinematics, $W+j e t(s)$ backgrounds are quite efficiently suppressed and have small contribution to our final sample.

- $\gamma+j e t(s)$ :

Relevant only for $\tau_{e} \tau_{h}$ channel, represents the source of background events with an electron from non-removed conversion (probably with one very soft leg). All event cuts applied as for signal, the selected electron is required to be a conversion leg.

These control samples allow us to test the fake rate predictions in an environment that is compatible with the signal region. Tau contamination and backgrounds cross-contamination in these samples are minimal.

We compare the several distributions to check the consistency of the predicted and observed fake taus. The shape of the distributions should not be compared with the expectation in our final sample. This is especially true for $W+$ jet $(s)$ sample selected from data. In this case the topology is quite different from our signal due to redefinition of the $\zeta$ region.

The comparisons are presented in the following plots. he distributions of the predicted/observed tau fakes agree within the assigned uncertainties. 
The observed distributions give us confidence in the prediction of the effective mass shape for $j e t \rightarrow \tau$ fakes. The predicted number of fakes taus is well contained between the upper and lower bands (half-spread of the order of $15 \%$ ).

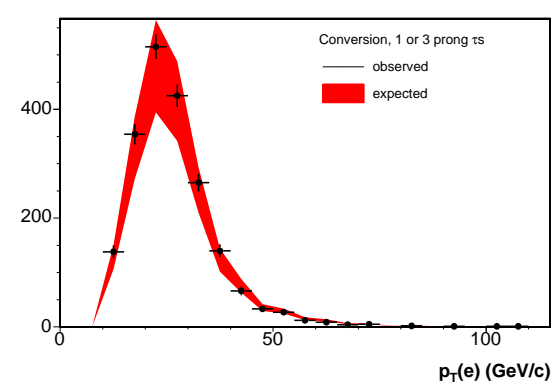

(a) Conversion electron $E_{T}$

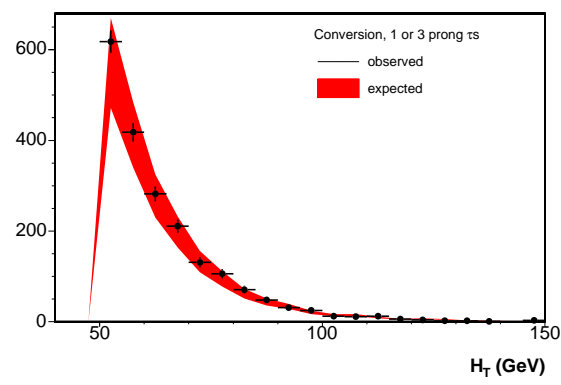

(c) $\tilde{H}_{T}$

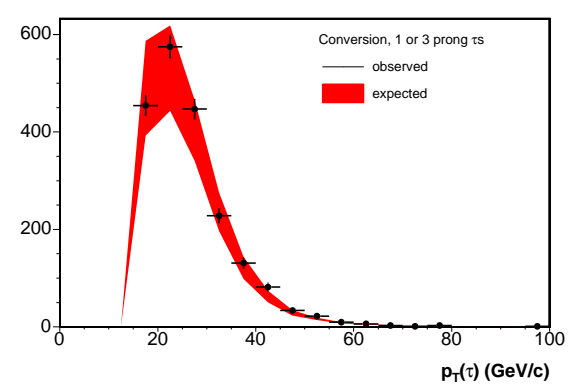

(b) Fake tau $p_{T}$

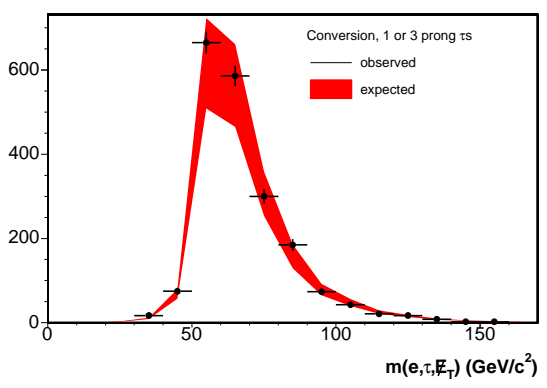

(d) $m_{v i s}$

Figure 7.1: Consistency checks of $j e t \rightarrow \tau$ fake predictions using the $\gamma+j e t$ sample. The upper and lower bounds come from using the fake rates from the highest and second-highest $E_{T}$ jet in the event. 


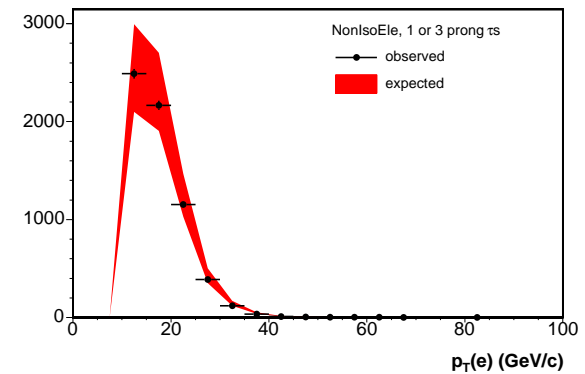

(a) Non-isolated electron $E_{T}$

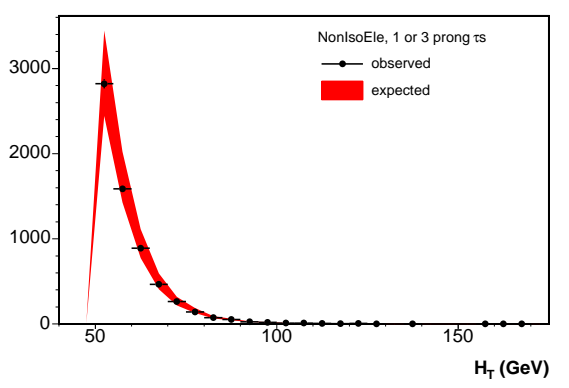

(c) $\tilde{H}$

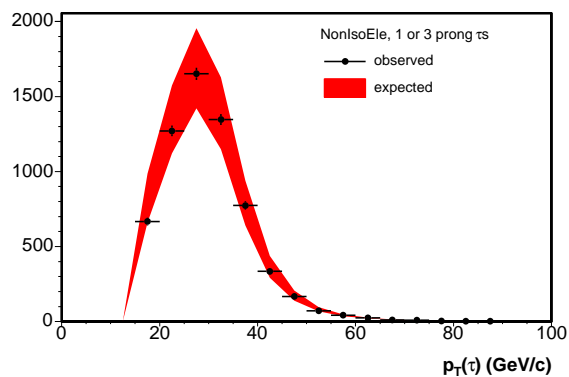

(b) Fake tau $p_{T}$

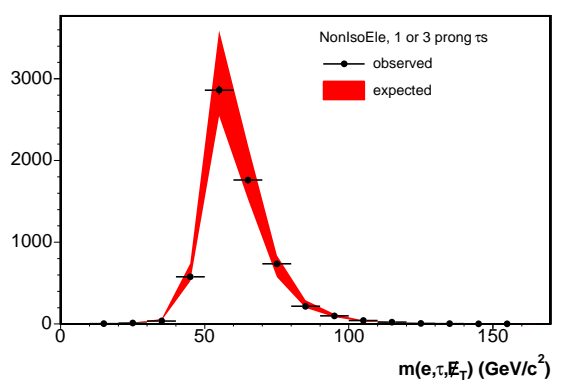

(d) $m_{\text {vis }}$

Figure 7.2: Consistency checks of $j e t \rightarrow \tau$ fake predictions using the non-isolated electron sample. The upper and lower bounds come from using the fake rates from the highest and second-highest $E_{T}$ jet in the event. 


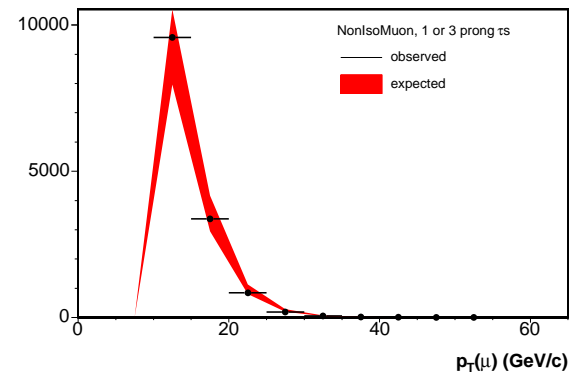

(a) Non-isolated electron $E_{T}$

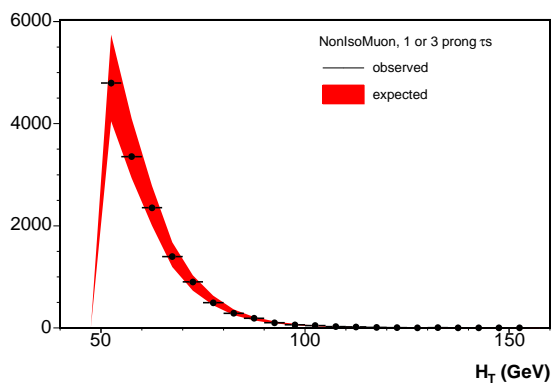

(c) $\tilde{H}$

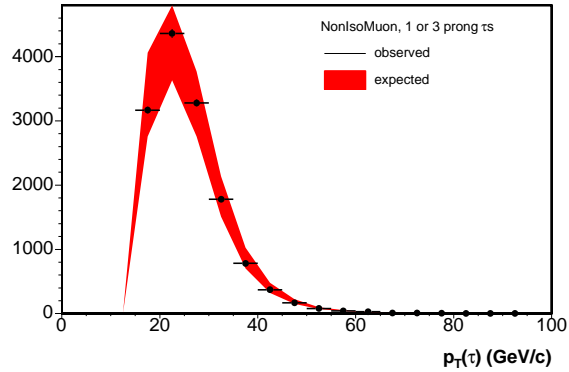

(b) Fake tau $p_{T}$

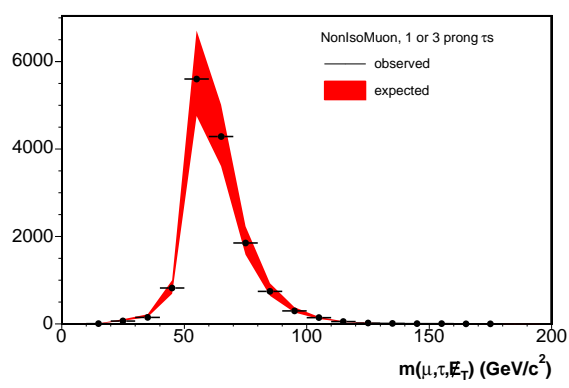

(d) $m_{v i s}$

Figure 7.3: Consistency checks of $j e t \rightarrow \tau$ fake predictions using the non-isolated muon sample. The upper and lower bounds come from using the fake rates from the highest and second-highest $E_{T}$ jet in the event. 


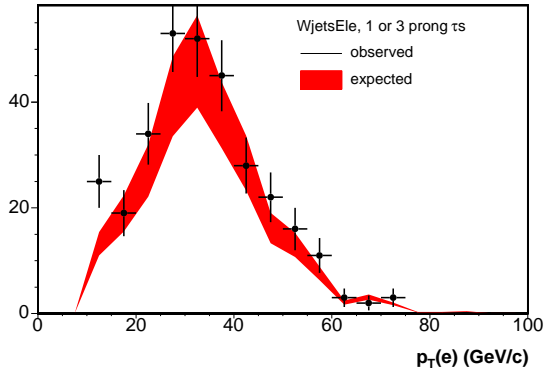

(a) Electron $E_{T}$

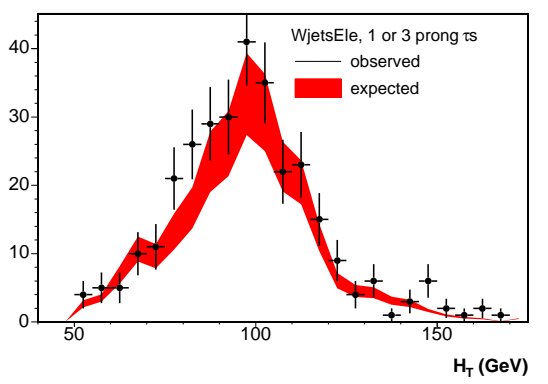

(c) $\tilde{H}_{T}$

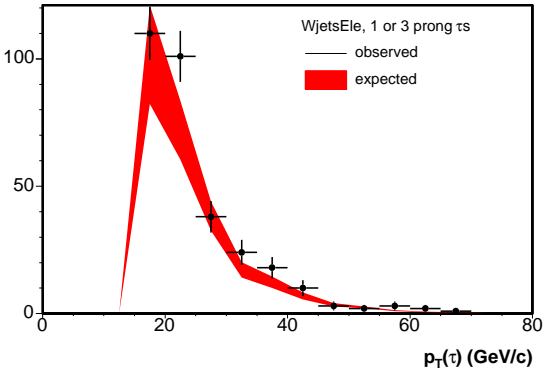

(b) Fake tau $p_{T}$

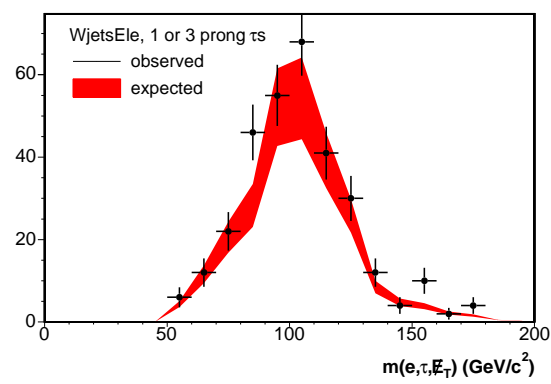

(d) $m_{\text {vis }}$

Figure 7.4: Consistency checks of $j e t \rightarrow \tau$ fake predictions using region dominated by $W+j e t(s), W \rightarrow e \nu$. The upper and lower bounds come from using the fake rates from the highest and second-highest $E_{T}$ jet in the event. 


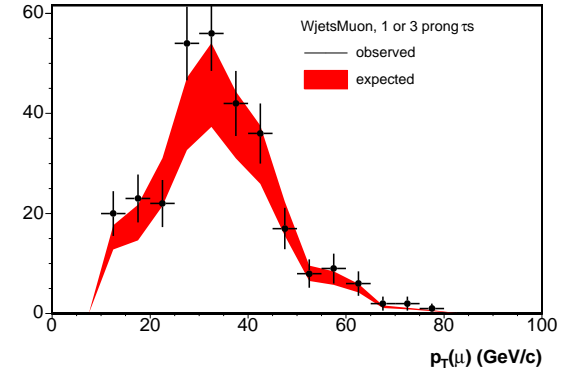

(a) Muon $p_{T}$

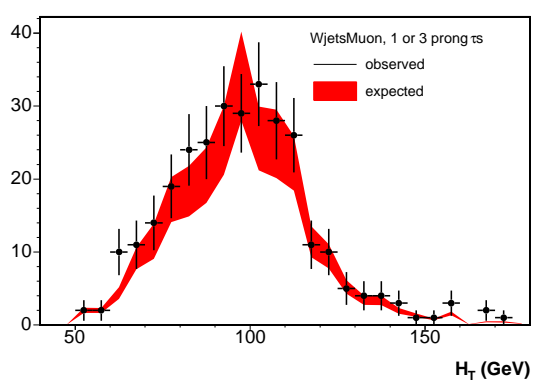

(c) $\tilde{H}_{T}$

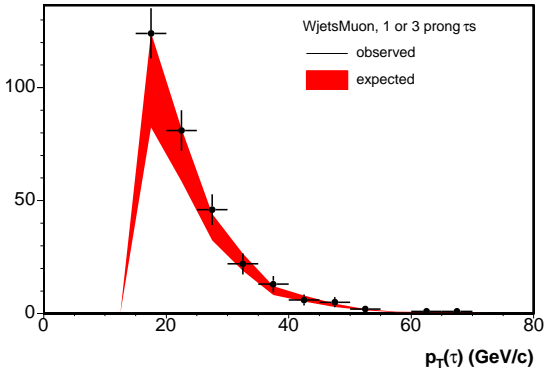

(b) Fake tau $p_{T}$

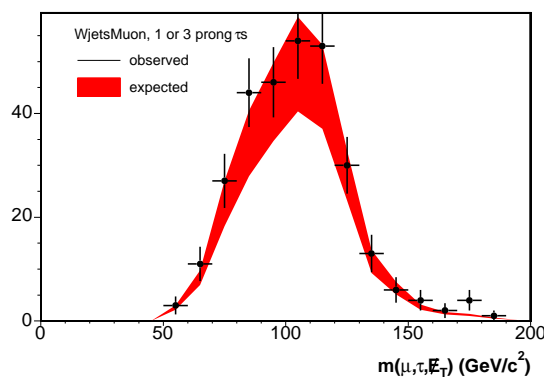

(d) $m_{v i s}$

Figure 7.5: Consistency checks of $j e t \rightarrow \tau$ fake predictions using region dominated by $W+j e t(s), W \rightarrow \mu \nu$. The upper and lower bounds come from using the fake rates from the highest and second-highest $E_{T}$ jet in the event.

\subsection{Event cuts}

Apart from the irreducible background from $Z / \gamma^{*} \rightarrow \tau \tau$ the major other backgrounds in this analysis are $Z / \gamma^{*} \rightarrow l l, W \rightarrow l \nu+j e t(s)(l=e, \mu)$, and "QCD" $(p \bar{p} \rightarrow j e t s)$. The event cuts are designed as a compromise between effective suppression of the major reducible backgrounds and maintaining sufficient signal efficiency. Other backgrounds, such as $t \bar{t}$ and di-boson events are taken into account in the analysis (and are also suppresses by some of the cuts), but due to their 
small contribution played limited role in designing the cuts.

\subsubsection{General requirements}

The two leptons must come from the same interaction: $\left|z_{0}^{(1)}-z_{0}^{(2)}\right|<5 \mathrm{~cm}$. To increase signal detection efficiency we do not require a reconstructed primary vertex in the event. The $p \bar{p}$ interaction point $\left|z_{0}^{i n t}\right|$ is taken as the average $z_{0}$ 's of the two leptons: $z_{0}^{i n t}=0.5 \times\left(z_{0}^{(1)}+z_{0}^{(2)}\right)$, and must be in the luminous region: $\left|z_{0}^{i n t}\right|<60 \mathrm{~cm}$. The electron and muon are required to have opposite charge: $Q^{(1)} Q^{(2)}=-1$.

\subsection{2 $\zeta$ cut}

The " $\zeta$ cut" in an attempt to discriminate events with $E_{\mathrm{T}}^{\text {miss }}$ that are not consistent with a particle decaying to two taus. It is targeted at di-boson, $W \rightarrow l \nu+j e t(s)$, but also helps to suppress QCD, and $t \bar{t}$ events.

We define a bisection axis $\zeta$ in the transverse plane for the directions of the visible tau decay products (in this case the electron and muon) -see Figure 7.6. The transverse momentum of $\phi$ (or any other particle decaying to two taus) is

$$
\boldsymbol{P}_{T}^{\phi}=\boldsymbol{P}_{T}^{v i s}\left(\tau_{1}\right)+\boldsymbol{P}_{T}^{v i s}\left(\tau_{2}\right)+\boldsymbol{E}_{\mathbf{T}}^{\text {miss }}
$$

where we assume that $\boldsymbol{E}_{\mathbf{T}}^{\text {miss }}$ is the sum of the transverse momenta of the neutrinos from tau decays. The transverse momentum of the tau visible products is

$$
\boldsymbol{P}_{T}^{v i s \tau}=\boldsymbol{P}_{T}^{v i s}\left(\tau_{1}\right)+\boldsymbol{P}_{T}^{v i s}\left(\tau_{2}\right)
$$

The projections of $\boldsymbol{P}_{T}^{\phi}$ and $\boldsymbol{P}_{T}^{v i s \tau}$ onto the defined $\boldsymbol{\zeta}$ axis are

$$
P_{\zeta}^{\phi}=\boldsymbol{P}_{T}^{\phi} \cdot \zeta
$$

and

$$
P_{\zeta}^{v i s \tau}=\boldsymbol{P}_{T}^{v i s \tau} \cdot \boldsymbol{\zeta}
$$

respectively. 


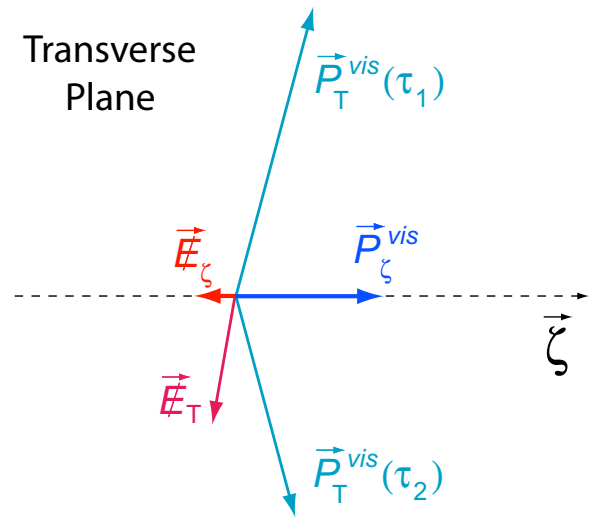

Figure 7.6: Illustration of the definition of parameters used in the $\zeta$ cut.

Figure 7.7 shows the $P_{\zeta}$ vs $P_{\zeta}^{v i s \tau}$ distribution for $\phi \rightarrow \tau \tau$, and $W \rightarrow l \nu+j e t(s)$ MC events. The distributions are plotted after applying the other event cuts. The graphical cut shown on these plots results in small efficiency loss and substantial background suppression.

The rational behind this cut is simple: both the neutrinos and the visible decay products from tau decays go at small angles from the initial tau direction. Therefore, the sum of the neutrino's momenta should not go opposite to the direction of the sum of visible products. The defined acceptance region implicitly takes into account MET resolution. The cut placement is determined by inspecting the predicted distributions and minimizing the signal losses. Figure 7.7 shows the effect of the cut on signal (Higgs) and several of the backgrounds.

Given the choice to apply a 2D cut, we can only show the data/MC comparison of the projections of the events on the two axis. The plots in Figure 8.1 (section 8.1) show the distributions before the application of the bisector-related cuts.

\subsubsection{Suppression of QCD backgrounds.}

Jets are suppressed to a significant degree by the lepton ID cuts and the isolation requirements. However, at low $E_{T}$ their number is so large that event cuts are 


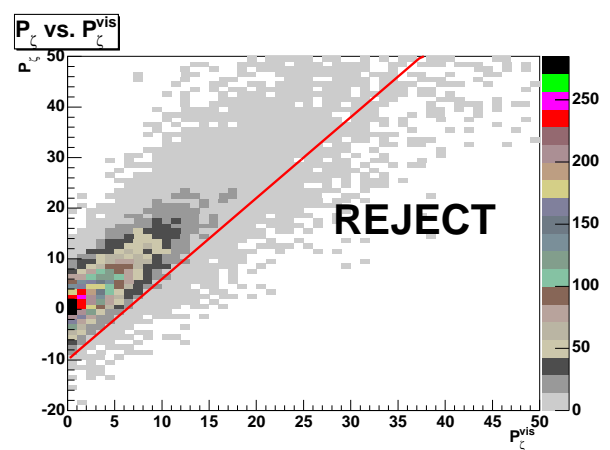

(a) $A \rightarrow \tau \tau \mathrm{MC}$

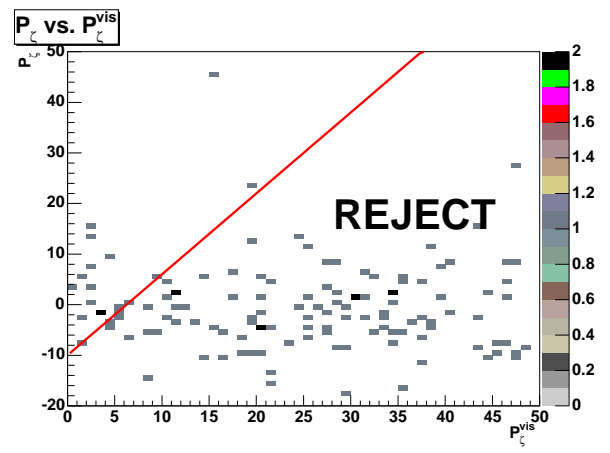

(b) $W \rightarrow e \nu \mathrm{MC}$

Figure 7.7: Effect of the $\zeta$ cut.

needed to clean up the sample.

For the $\tau_{l} \tau_{\text {had }}$ we define $H_{T}=\left|p_{T}^{l}\right|+\left|p_{T}^{\tau}\right|+E_{\mathrm{T}}^{\text {miss }}$. Previously we placed a uniform cut of $H_{T}>50 \mathrm{GeV}$. In this analysis we apply different cuts, based on track multiplicity of the tau candidates. 3-prong tau decays have larger contamination from misidentified jets and we raise the threshold to $H_{T}>55 \mathrm{GeV}$. For 1-prong tau decays in the $\tau_{e} \tau_{\text {had }}$ channel we keep the cut at $50 \mathrm{GeV}$. The jet contamination in 1-prong decays in the $\tau_{\mu} \tau_{\text {had }}$ is the smallest, and we use a cut of $H_{T}>45 \mathrm{GeV}$ to recover some efficiency in the low-mass Higgs region. The cuts are placed based 
on the inspection of the distributions of same-sign events in the data.

For the $\tau_{e} \tau_{\mu}$ the jet backgrounds can be reduced significantly with sufficiently high lepton $p_{T}$. However, this leads to a loss in signal efficiency. As compromise require $\left|E_{T}^{e}\right|+\left|p_{T}^{\mu}\right|>30 \mathrm{GeV}$, that allows us to keep the jet backgrounds under control. Figure 7.8 shows the distributions for like-sign events in the data, and the lowestmass Higgs boson $\left(m_{A}=90 \mathrm{GeV}\right)$ considered in this search, and Higgs with mass $m_{A}=200 \mathrm{GeV}$. In addition for approximating the shape, like-sign events also give us an approximate estimate of the number of background events that will enter the sample. The cut value is chosen to cut out the bulk of the exponentially increasing background events.

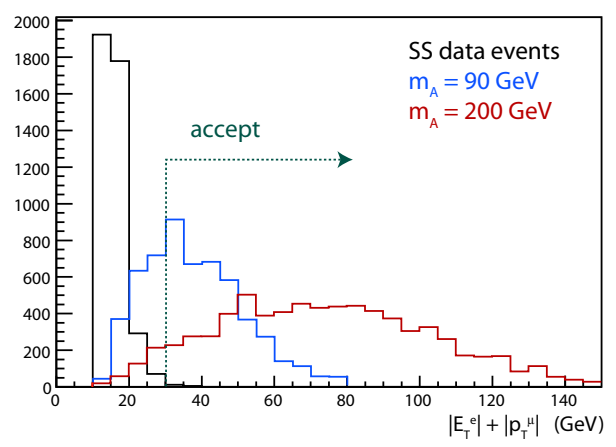

Figure 7.8: Effect of the $\left|E_{T}^{e}\right|+\left|p_{T}^{\mu}\right|$ cut on like-sign data events and Higgs signal $\left(m_{A}=90,200 \mathrm{GeV}\right)$. The normalization of the Higgs signal is arbitrary.

\subsection{4 $Z \rightarrow \mu \mu$ removal}

In the $\tau_{\mu} \tau_{\text {had }}$ channel we apply a cut to reduce the background from $Z \rightarrow \mu \mu$ events. We veto events with 1-prong tau decays if the invariant mass of the tau track and the muon is within $10 \mathrm{GeV}$ of $m_{Z}$ in cases when the track points to the $\phi$ cracks, or the calorimeter cluster $E_{T}$ is smaller than the maximum of $10 \mathrm{GeV}$, and $40 \%$ of the seed track $p_{T}$. 


\subsection{Backgrounds and estimation methods}

\subsection{1 $Z \rightarrow l l$}

This group includes $Z \rightarrow \tau \tau$ decays to the same final states as the signal. Apart from di-tau mass (and related parameters) these events are practically indistinguishable from the signal ${ }^{1}$. This is the largest expected background for this analysis. We do not make any attempt to suppress the $Z$ background. Any differences in di-tau mass related parameters are used at the time of signal extraction/ limit setting procedure. $Z \rightarrow e e$ and $Z \rightarrow \mu \mu$ also contribute some background events due to particle misidentification. These backgrounds are also estimated using MC samples. The contribution of these backgrounds is estimated using the MC simulated events. The samples are normalized to the CDF measurement of the $Z$ production cross section in $Z \rightarrow e e$ (lepton universality is assumed).

\subsubsection{Di-boson, $W+\gamma$, and $t \bar{t}$}

These are small backgrounds (due to small production cross sections). The final states contains the particles expected in our signal final state (in the $W+\gamma$ case there must be an unremoved photon conversion). These backgrounds are substantially suppressed by the " $\zeta$ cut". All these backgrounds are estimated using MC samples.

\subsubsection{Backgrounds with misidentified or non-isolated $e$ or $\mu$ in the $\tau_{e} \tau_{\mu}$ detection mode}

The leptons in this group come either from a misidentified particle in a jet, or a real lepton contained in a jet. We do not separate these two sources and use a procedure based on the selecting events with non-isolated leptons to estimate the contamination in our final sample and predict the shapes in the distributions of interest.

\footnotetext{
${ }^{1}$ In principle, there are some differences due to boson spin resulting in differences in $\tau$ polarizations. This information will be used in an update of the analysis with the use of a NN for signal selection
} 
We use the same $p_{T}$ thresholds and isolation requirements for electrons and the muons that allows us to treat them in a symmetric way. For each lepton we define a sideband regions in terms of track isolation that is separated from the signal region $\left(I_{i s o}^{t r k}<2 \mathrm{GeV}\right): 4<I_{i s o}^{t r k}<10 \mathrm{GeV}$. The other lepton is required to be isolated. The area of each sideband is larger than the size of the signal region to increase the statistics of the selected events. The sum of the events in the two lepton sidebands are used to approximate the background shapes for events that are contained in the signal region. The sample is normalized to account for the different area in the sidebands. The distribution of track isolation is not guaranteed to be flat in signal and sideband regions. This effect is handled using information from the like-sign events: we estimate the expected number of like-sign events using the sidebands and compare with the observed number of isolated like-sign $e \mu$ pairs (after subtracting contributions from the other background sources). The ratio is used as an additional scale factor $f_{s b}$ for the background in the opposite-sign sample. The distributions in the sidebands of like-sign electrons and muons is shown in Figure 7.9. From these distributions we obtain $f_{s b}=1.15 \pm 0.20$.

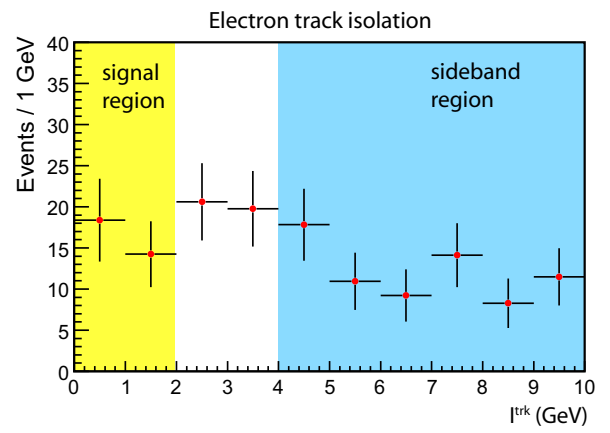

(a) Electron isolation

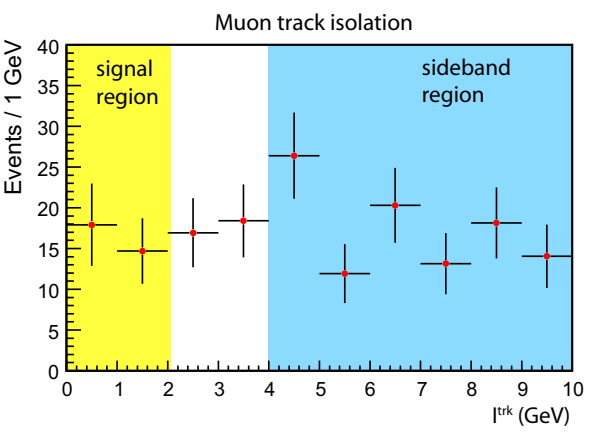

(b) Muon isolation

Figure 7.9: Track isolation for electrons and muons in same-sign events passing all other selection criteria.

This procedure fully accounts for multi-jet backgrounds. In this case both leptons originate from jets. Another source we have to consider is $W+$ jets, where only one of the leptons is misidentified, and the other one is from $W \rightarrow l \nu$. For this case the above procedure will account only for half of the events from this source 
(each sideband should contain the respective events with one misidentified lepton, however when taking the average of the two we allow half of these $W+$ jets events). $W+$ jets events are effectively suppressed by the $\zeta$-cut and we their contribution to the final sample is negligible. To confirm this hypothesis we use inclusive $W$ MC samples. We do not expect the MC to reliably predict the absolute number of background events and instead of scaling the MC samples to the data luminosity we normalize to the excess of events in the data in a control region.

We select $W \rightarrow \mu \nu$ candidates with $p_{T}^{\mu}>25 \mathrm{GeV}$ and $E_{\mathrm{T}}^{\mathrm{miss}}>25 \mathrm{GeV}$ and look for an additional isolated electron candidate. We ask that the events are not in our signal region by reversing the $\zeta$-cut. We observe a total of 40 events in the data sample with expected backgrounds (other than $W+$ jets) of 35.8 events. We repeat the same procedure for $W \rightarrow e \nu$ and find excess of 2.8 events in 31 observed events in the data.

The MC scale factors have large uncertainty (due to data statistics in the normalization regions), but these comparisons point to the smallness of $W+$ jets backgrounds. Indeed, after scaling the MC we predict that there are $2 W+j e t$ events in the signal region that are not accounted for by the sideband subtraction method. This contribution is very small compared to the total background estimate and these events are not treated as a separate background source. Instead, we add them to the rest of misidentified/non-isolated lepton backgrounds.

\subsubsection{Backgrounds from misidentified $j e t \rightarrow \tau_{h a d}$ in the $\tau_{l} \tau_{\text {had }}$ detection modes}

There are three quite different processes that contribute to this group:

- $W \rightarrow l \nu+j e t(s):$ one of the jets fakes a hadronic tau

- $\gamma+$ jet: the photon undergoes conversion with one soft/undetected leg, resulting in reconstruction of an isolated electron; the jet fakes a hadronic tau

- $p \bar{p} \rightarrow n$ jets: one jet fakes a tau, another one fakes an $e / \mu$ 
In the previous searches we have applied two different methods for $j e t \rightarrow \tau_{\text {had }}$ misidentification: fake rate method, and method based on combined use of same sign events and $W+$ jets MC. In this iteration we will apply both. At this time we use the estimates of the former as the "default" method, and the prediction of the latter as a cross-check. Since the samples are statistically independent, on can combine the results to obtain a smoother background shape (will follow soon).

The fake rate method assumes that the jets from the above sources have similar properties and that differences are covered by assigning systematic uncertainties $(\sim 15 \%)$. The challenge of applying this method to events selected with the "lepton+track" triggers is that the tau candidates are already quite isolated (in terms of tracks). Consequently, when applying fake rates we have to account for the substantial "contamination" with real taus. We have developed a method that accounts for this contamination, and it has been successfully used in our previous analysis [97] The following is an outline of the method.

Let us start with a simplified case, and consider an initial sample passing loose tau cuts, and a final sample produced after applying the tight cuts. The number of real taus and $j e t \rightarrow \tau$ fakes that survive this transition depend on the efficiency and fake rate (calculated with respect to the loose objects).

Let $\hat{N}$ be the number of tau candidates passing the loose tau cuts, and denote the number of candidates passing the tight tau cuts by $N$. There are three sources that contribute to the observed events: real taus, leptons $(l=e, \mu)$, and "jets". This is reflected in the following set of equations

$$
\begin{gathered}
\hat{N}=\hat{N}^{\tau}+\hat{N}^{j e t}+\hat{N}^{l} \\
N=N^{\tau}+N^{j e t}+N^{l} \\
N^{\tau}=\epsilon \hat{N}^{\tau} \\
N^{j e t}=f \hat{N}^{j e t},
\end{gathered}
$$

where the last two expressions are the definitions of relative efficiency and fake rate. 
Then it is easy to show that the jet background can be written as

$$
N^{j e t}=\frac{f}{\epsilon-f}[\epsilon \hat{N}-N]-C_{l},
$$

where $C_{l}$ is a correction for $e, \mu$ contributions (which is small for the considered processes). It has the form

$$
C_{l}=\frac{f}{\epsilon-f}\left[\epsilon \hat{N}^{l}-N^{l}\right]
$$

where the leptons passing as loose and tight taus can be obtained from MC simulation.

If we take into account that efficiency and fake rate are actually functions of the parametrization variables (whatever they might be) and write the equations for infinitesimally small regions in parameter space, we get the same expressions for the fake tau density in terms of the event densities $\hat{n}$ and $n$ (instead of number of events)

$$
n^{j e t}(\Omega)=\frac{f(\Omega)}{\epsilon(\Omega)-f(\Omega)}[\epsilon(\Omega) \hat{n}(\Omega)-n(\Omega)]-c_{l},
$$

where $\Omega$ denotes a point in the efficiency/fake rate parametrization space. The densities $\hat{n}(\Omega)$ and $n(\Omega)$ are given by

$$
\begin{aligned}
& \hat{n}(\Omega)=\sum_{i}^{\hat{N}} \delta\left(\Omega-\Omega_{i}\right) \\
& n(\Omega)=\sum_{i}^{N} \delta\left(\Omega-\Omega_{i}\right) .
\end{aligned}
$$

To obtain the number of $j e t \rightarrow \tau$ fakes in the final sample we substitute the above densities in the expression for $n^{\text {jet }}(\Omega)$ and integrate over the parameter space. The QCD estimation reduces to a sum over all loose taus which enter with weight

$$
w_{i}^{\overline{I D}}=\frac{f\left(\Omega_{i}\right) \epsilon\left(\Omega_{i}\right)}{\epsilon\left(\Omega_{i}\right)-f\left(\Omega_{i}\right)}
$$


if the candidate did not pass tight tau ID cuts, and

$$
w_{i}^{I D}=\frac{f\left(\Omega_{i}\right)\left(\epsilon\left(\Omega_{i}\right)-1\right)}{\epsilon\left(\Omega_{i}\right)-f\left(\Omega_{i}\right)}
$$

if it did. It is straight forward to apply the "lepton correction".

Using these weights we can obtain the distributions for various event variables.

We choose to parameterize the the fake rates and efficiencies in terms of track multiplicity, sum of the tau cluster $E_{T}$ and calorimeter isolation $E_{T}$, and $\eta_{\text {det }}$ of the tau candidate. The fake rates are obtained from jet samples as described in section 7.1 .

As can be see, the agreement between the predicted and observed tau fakes is quite good. One can argue that the observed fakes in the $W+$ jets control region are closer to the upper limit of our prediction. This is not surprising, since the fake rates reflect the quark/gluon jet composition in multi-jet events. We can account for this small deviation by using $W+$ jets $\mathrm{MC}$ events, normalized to account for the observed difference in the control region. Using this normalization we can obtain contribution to the signal region. This procedure allows us to bring the tau fakes estimated to the level of the central value of the expectation. These additional contributions are included in the table with the summary of the observed backgrounds.

\subsection{Signal selection efficiency}

The Higgs signal selection efficiency for the defined cuts is shown in Figure 7.10 for the three detection modes. The efficiency is defined with respect to Higgs $\rightarrow \tau \tau$ production, where both taus can decay in any mode. 


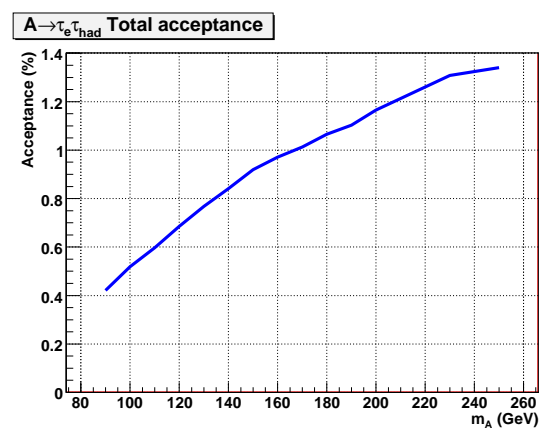

(a) $\tau_{e} \tau_{h a d}$

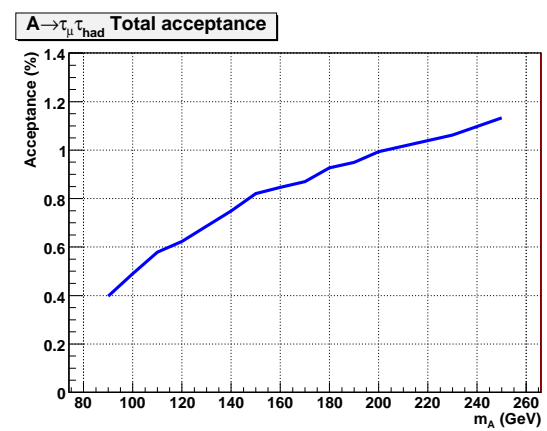

(b) $\tau_{\mu} \tau_{h a d}$

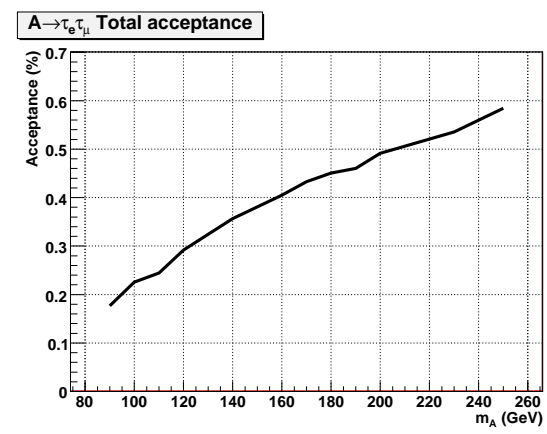

(c) $\tau_{e} \tau_{\mu}$

Figure 7.10: Signal selection efficiencies for the three detection modes as a function of Higgs mass $\left(m_{A}\right)$. 


\section{Chapter 8}

\section{Results}

\subsection{Observed Events}

The final events counts for the observed events are compared with background estimations in Table 8.1 and Table 8.2. The errors on both tables only account for statistical effects, unless otherwise quoted.

\begin{tabular}{lc}
\multicolumn{2}{l}{ CDF Run II Preliminary $1.8 \mathrm{fb}^{-1}$} \\
\hline source & events \\
\hline \hline$Z \rightarrow \tau \tau$ & $604.8 \pm 5.5$ \\
$Z \rightarrow e e$ & $1.5 \pm 0.4$ \\
$Z \rightarrow \mu \mu$ & $17.7 \pm 0.9$ \\
$W / Z \gamma$, di-bosons & $11.4 \pm 0.1$ \\
$t \bar{t}$ & $9.1 \pm 0.1$ \\
fake/non-ISO & $57.3 \pm 3.3$ \\
\hline Sum BG & $701.9 \pm 6.5$ \\
\hline DATA & 726 \\
\hline \hline
\end{tabular}

Table 8.1: Predicted backgrounds and observed events in the $\tau_{e} \tau_{\mu}$ after applying all selection cuts. The quoted errors are statistical only. 
The systematic errors are included as a gaussian constraints in the likelihood, one per source of uncertainty. Different uncertainties affect some or all the SM contributions to the final state, thus accounting for correlations between backgrounds.

CDF Run II Preliminary $1.8 \mathrm{fb}^{-1}$

\begin{tabular}{lcc}
\hline source & $\tau_{e} \tau_{\text {had }}$ & $\tau_{\mu} \tau_{\text {had }}$ \\
\hline \hline$Z \rightarrow \tau \tau$ & $1376.9 \pm 8.3$ & $1353.7 \pm 8.1$ \\
$Z \rightarrow l l$ & $69.7 \pm 2.0$ & $107.3 \pm 2.3$ \\
di-boson events & $4.3 \pm 0.1$ & $3.3 \pm 0.05$ \\
$t \bar{t}$ & $3.7 \pm 0.1$ & $3.0 \pm 0.07$ \\
total jet $\rightarrow \tau$ fakes & $466.5( \pm 15 \%$ sys $)$ & $283.6( \pm 15 \%$ sys $)$ \\
\hline Sum BG & 1921.1 & 1750.8 \\
\hline DATA & 1979 & 1666 \\
\hline \hline
\end{tabular}

Table 8.2: Predicted backgrounds and observed events in the $\tau_{e} \tau_{\text {had }}$ and $\tau_{\mu} \tau_{\text {had }}$ after applying all selection cuts. The quoted errors are statistical only.

The following plots show comparisons of various distributions of the predicted Standard Model processes and the observed events.

\subsection{Systematic uncertainties}

Systematic errors are the main source of uncertainty in this search. Some of these errors affect the overall normalization of the signal or background templates. This kind of systematic errors, so-called rate systematics, summarize effects that impact the number of events in the signal and background templates. However, the shapes of these templates are not affected by these sources of uncertainty.

Contrarily, some other systematic uncertainties make the shapes of the templates to vary. This second kind of systematic errors, named shape systematics, could also affect the overall number of events. Particle identification and event selection are affected by these uncertainties, thus changing the shapes and normalizations of the templates. These differences in shape are accounted for by producing sets of shifted templates in parallel to the nominal ones. 


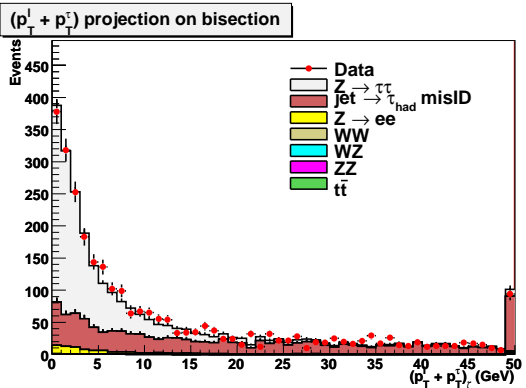

(a) $\tau_{e} \tau_{\text {had }}$ candidates: projections of $p_{T}$ of visible decay products on the bisector

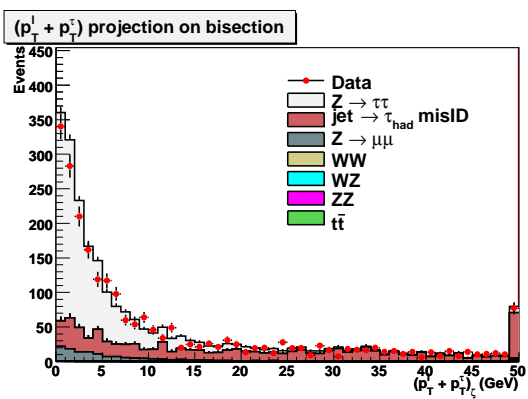

(c) $\tau_{\mu} \tau_{\text {had }}$ candidates: projections of $p_{T}$ of visible decay products on the bisector

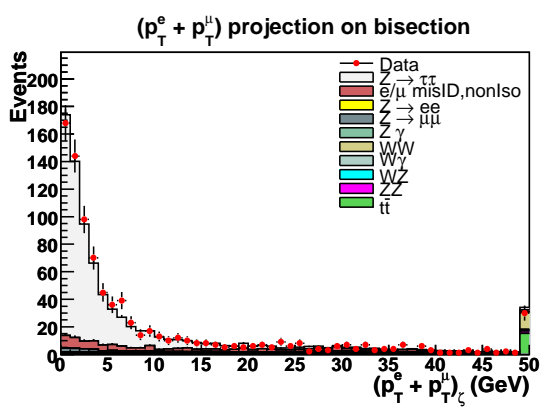

(e) $\tau_{e} \tau_{\mu}$ candidates: projections of $p_{T}$ of visible decay products on the bisector

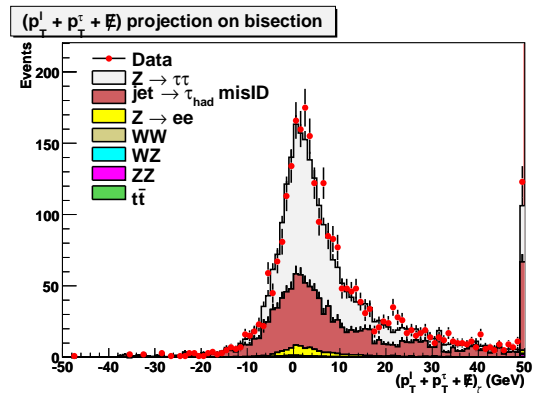

(b) $\tau_{e} \tau_{\text {had }}$ candidates: projections of $p_{T}$ of visible decay products and $E_{\mathrm{T}}^{\mathrm{miss}}$ on the bisector

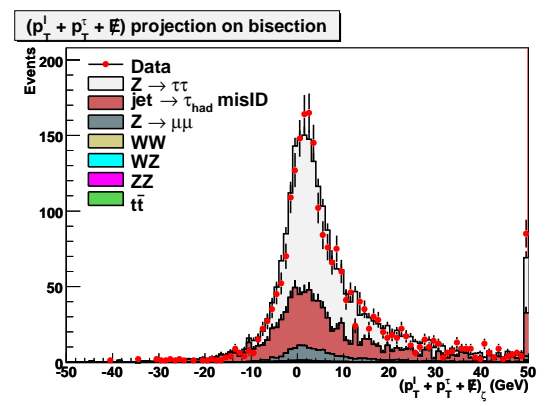

(d) $\tau_{\mu} \tau_{\text {had }}$ candidates: projections of $p_{T}$ of visible decay products and $E_{\mathrm{T}}^{\mathrm{miss}}$ on the bisector

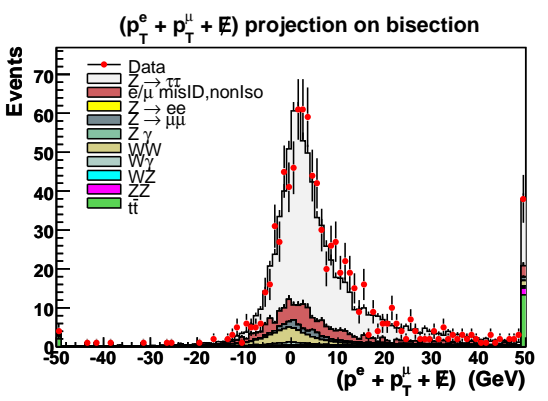

(f) $\tau_{e} \tau_{\mu}$ candidates: projections of $p_{T}$ of visible decay products and $E_{\mathrm{T}}^{\text {miss }}$ on the bisector

Figure 8.1: Distributions of data and predicted backgrounds for the projections on the two axes used in the "zeta-cut. The last bin includes the overflows. 


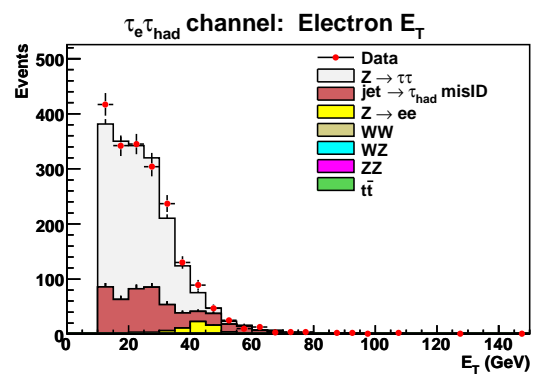

(a) Electron $E_{T}$

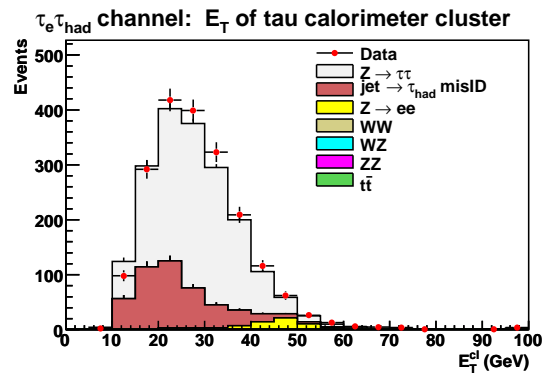

(c) Tau calorimeter cluster $E_{T}$

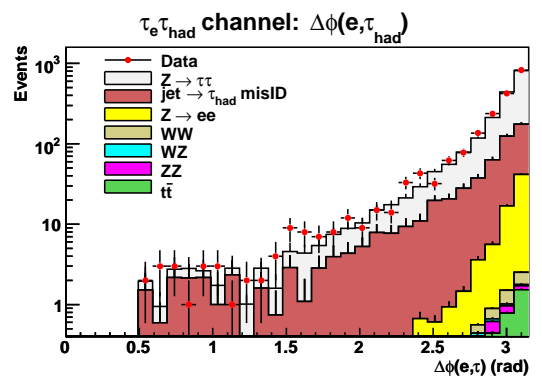

(e) $\Delta \phi$ between the tau and electron

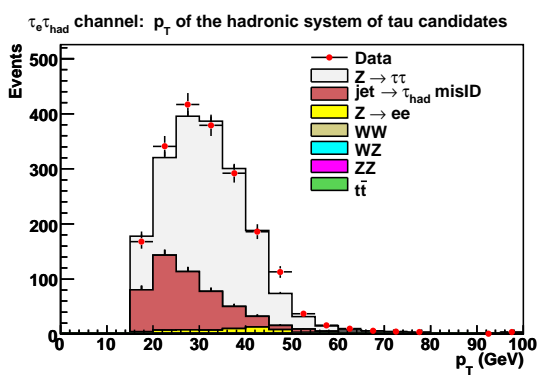

(b) Tau $p_{T}$

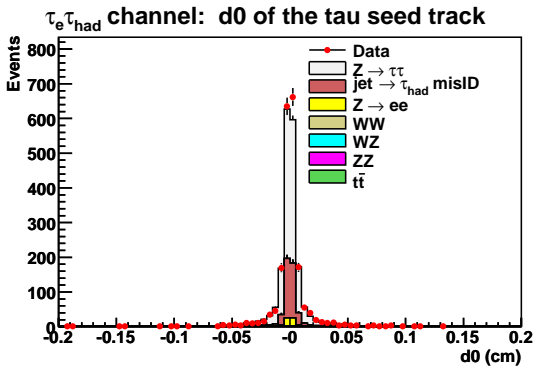

(d) Tau seed track impact parameter

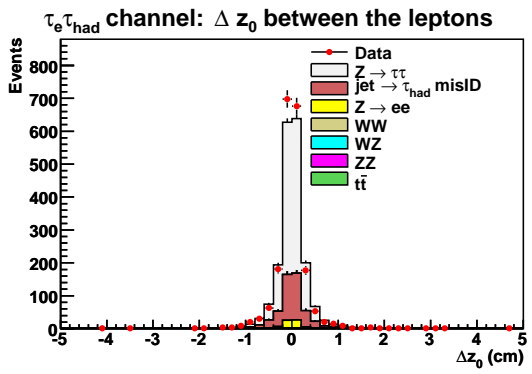

(f) $\Delta z_{0}$ between the tau and electron

Figure 8.2: Distributions for $\tau_{e} \tau_{h a d}$ candidate events. 


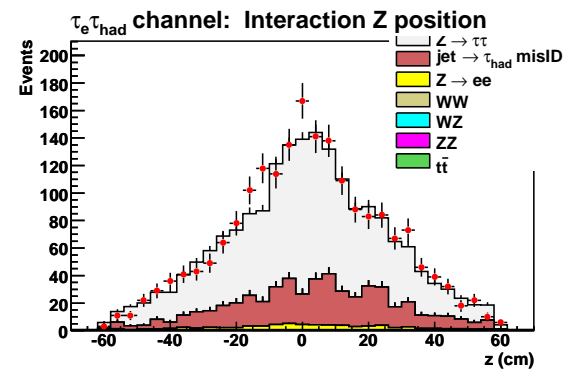

(a) $\left(z_{\tau}+z_{e}\right) / 2$

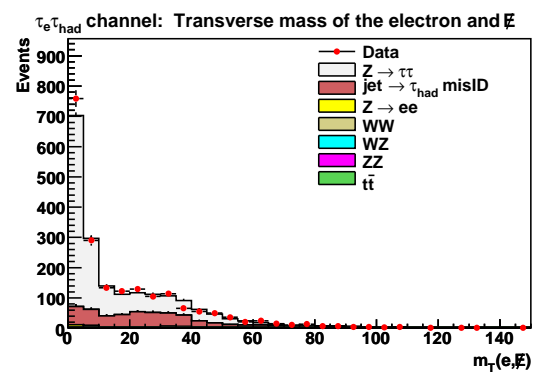

(c) Transverse mass of electron and $E_{\mathrm{T}}^{\text {miss }}$

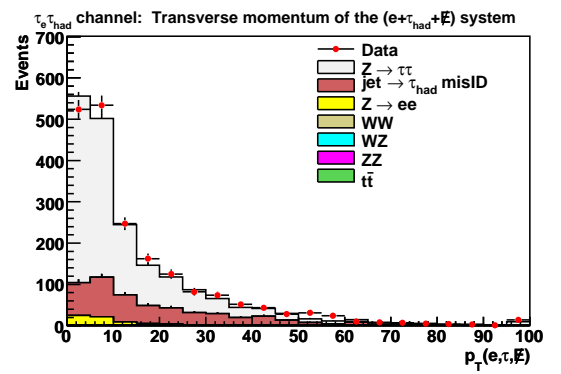

(e) $p_{T}$ of $e+\tau+E_{\mathrm{T}}^{\text {miss }}$

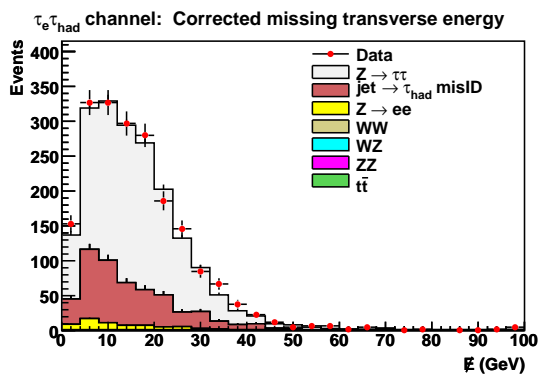

(b) Missing $E_{T}$

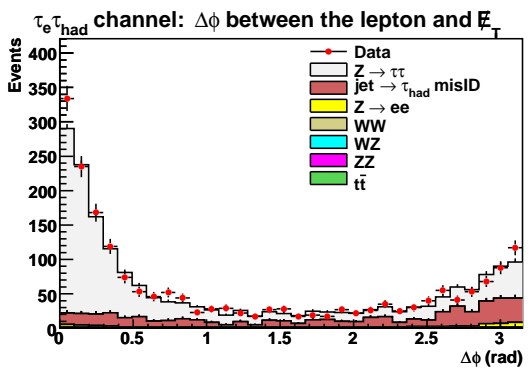

(d) $\Delta \phi\left(e, E_{\mathrm{T}}^{\text {miss }}\right)$

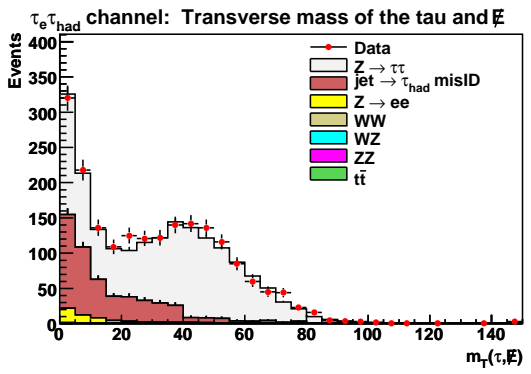

(f) Transverse mass of tau and $E_{\mathrm{T}}^{\mathrm{miss}}$

Figure 8.3: Distributions for $\tau_{e} \tau_{\text {had }}$ candidate events. 


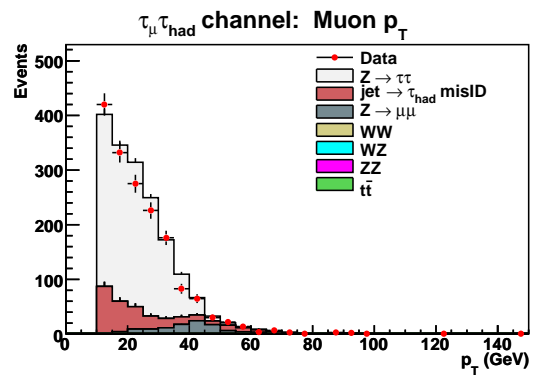

(a) Muon $p_{T}$

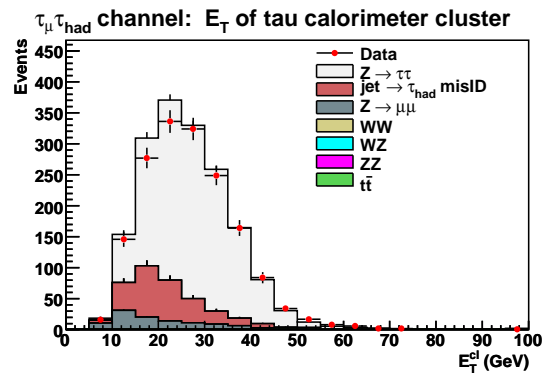

(c) Tau calorimeter cluster $E_{T}$

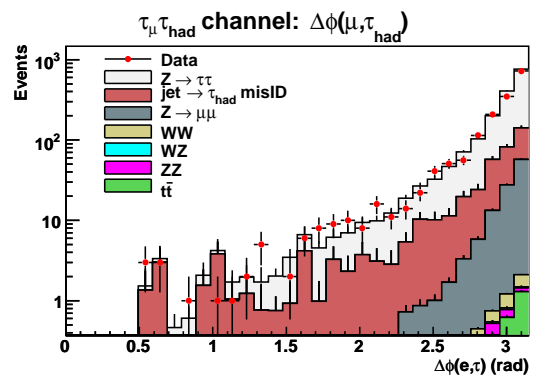

(e) $\Delta \phi$ between the tau and the muon

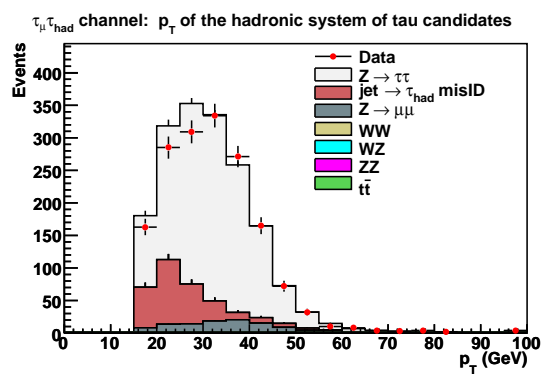

(b) Tau $p_{T}$

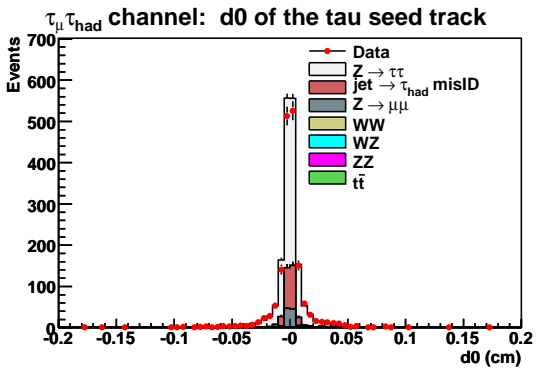

(d) Tau seed track impact parameter

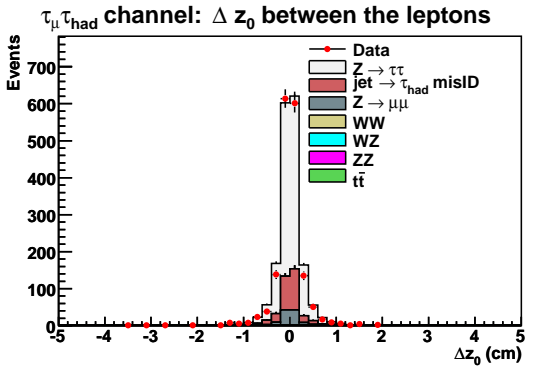

(f) $\Delta z_{0}$ between the tau and the muon

Figure 8.4: Distributions for $\tau_{\mu} \tau_{h a d}$ candidate events. 


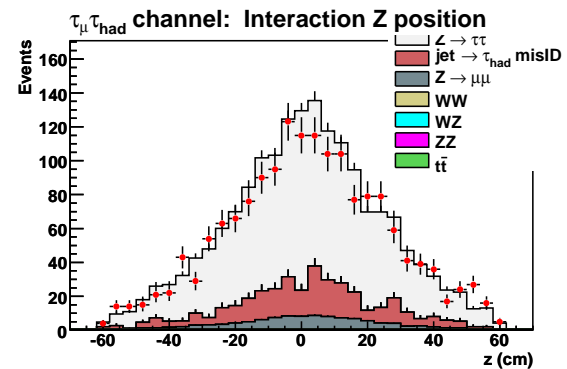

(a) $\left(z_{\tau}+z_{\mu}\right) / 2$

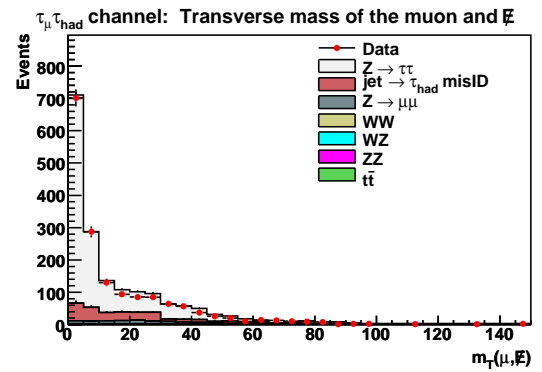

(c) Transverse mass of muon and $E_{\mathrm{T}}^{\text {miss }}$

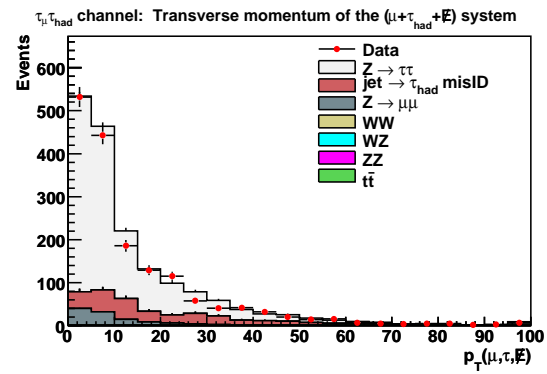

(e) $p_{T}$ of $\mu+\tau+E_{\mathrm{T}}^{\mathrm{miss}}$

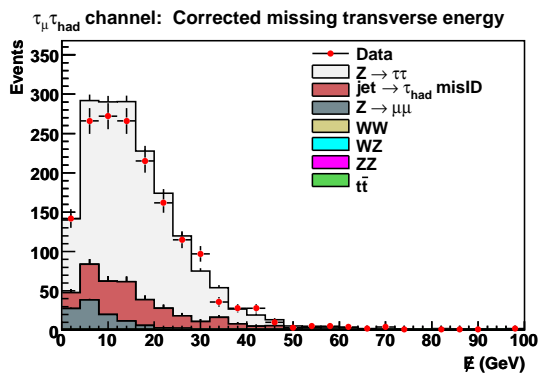

(b) Missing $E_{T}$

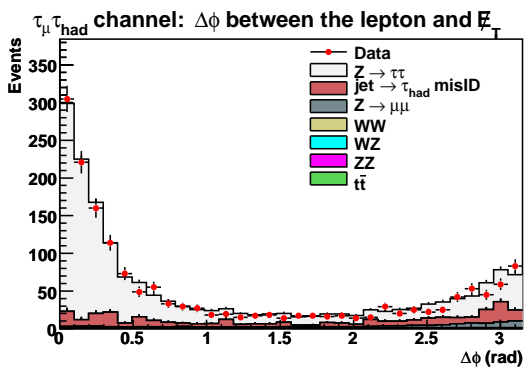

(d) $\Delta \phi$ between the muon and $E_{\mathrm{T}}^{\text {miss }}$

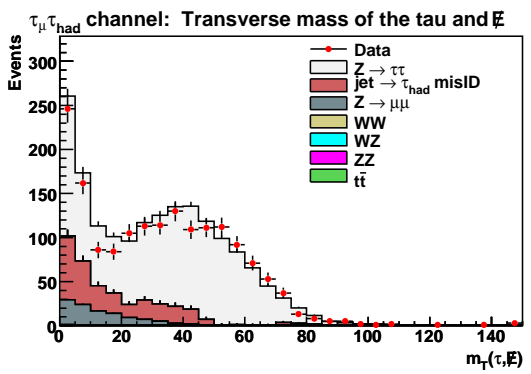

(f) Transverse mass of tau and $E_{\mathrm{T}}^{\mathrm{miss}}$

Figure 8.5: Distributions for $\tau_{\mu} \tau_{\text {had }}$ candidate events. 


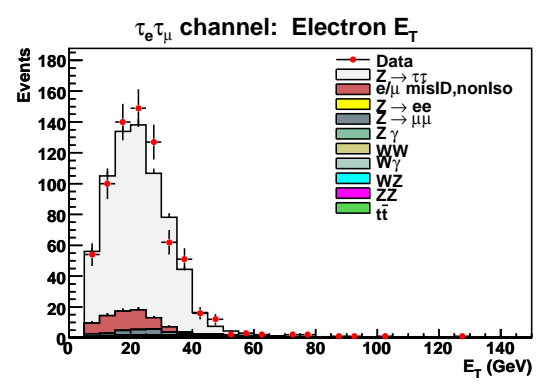

(a) Electron $E_{T}$

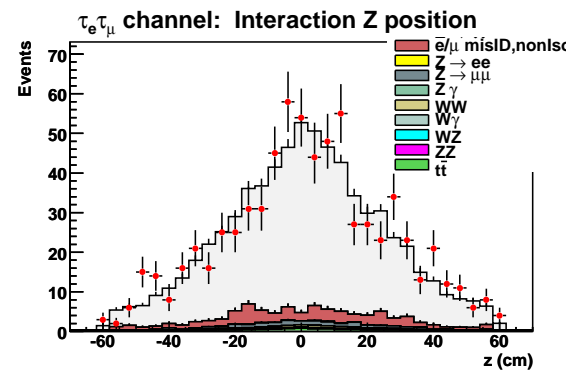

(c) $\left(z_{0}^{e}+z_{0}^{\mu}\right) / 2$

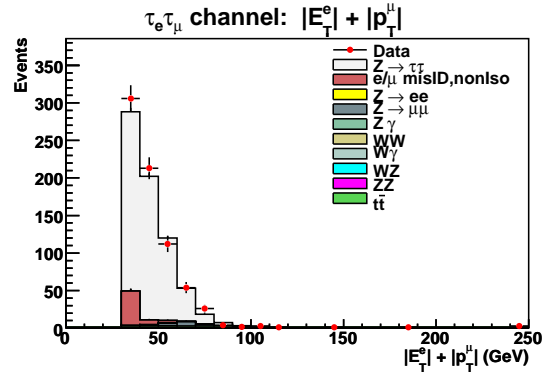

(e) Scalar sum of muon $p_{T}$ and electron $E_{T}$

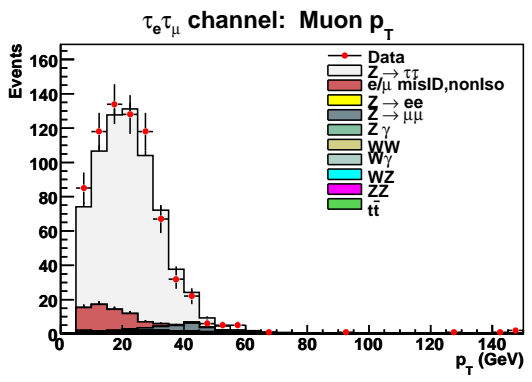

(b) Muon $p_{T}$

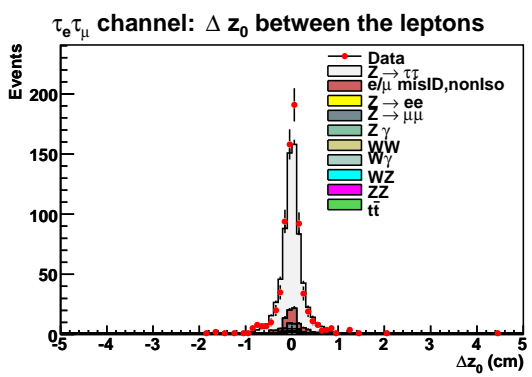

(d) $\Delta z_{0}$ between the electron and muon

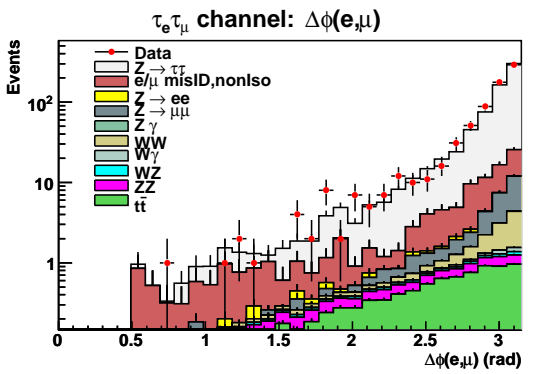

(f) $\Delta \phi$ between the electron and muon

Figure 8.6: Distributions for $\tau_{e} \tau_{\mu}$ candidate events. 


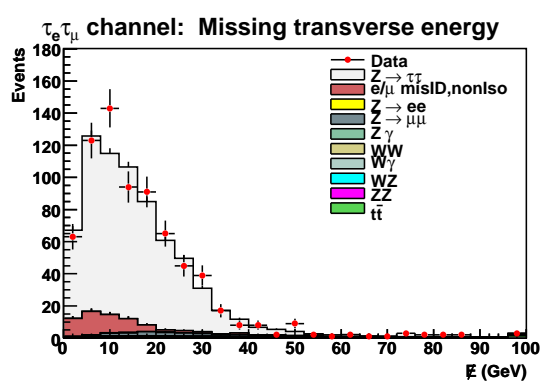

(a) Missing $E_{T}$

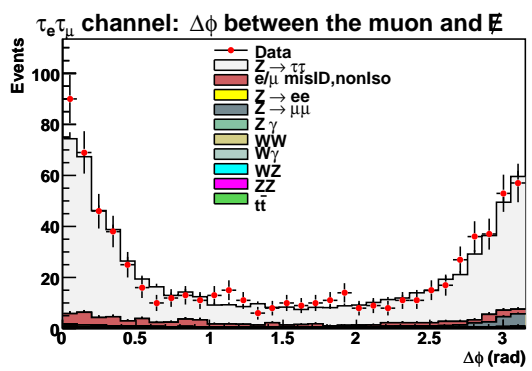

(c) $\Delta \phi$ between the muon and $E_{\mathrm{T}}^{\mathrm{miss}}$

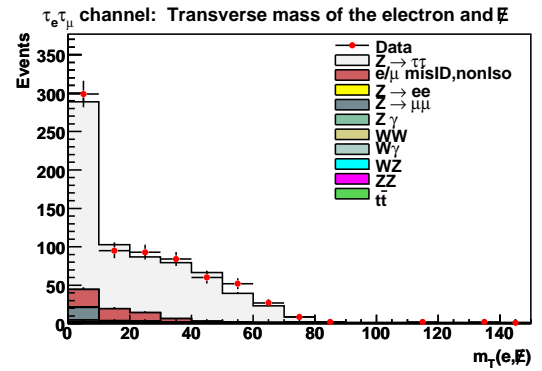

(e) Transverse mass of the electron and $E_{\mathrm{T}}^{\text {miss }}$

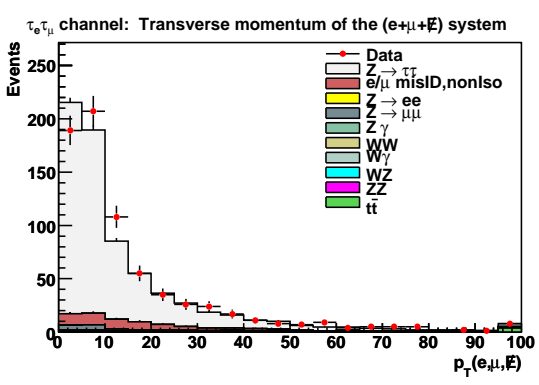

(b) $p_{T}$ of $e+\mu+E_{\mathrm{T}}^{\text {miss }}$

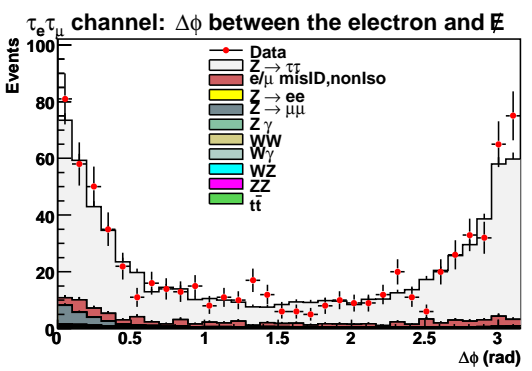

(d) $\Delta \phi$ between the electron and $E_{\mathrm{T}}^{\text {miss }}$

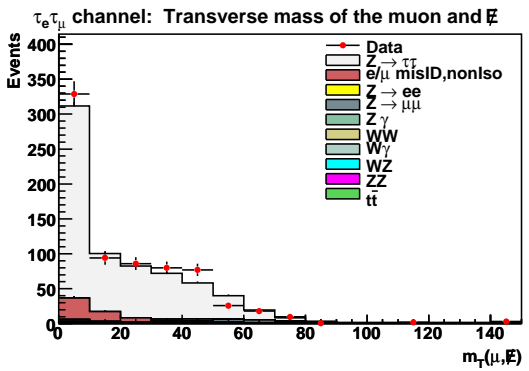

(f) Transverse mass of the muon and $E_{\mathrm{T}}^{\text {miss }}$

Figure 8.7: Distributions for $\tau_{e} \tau_{\mu}$ candidate events. 


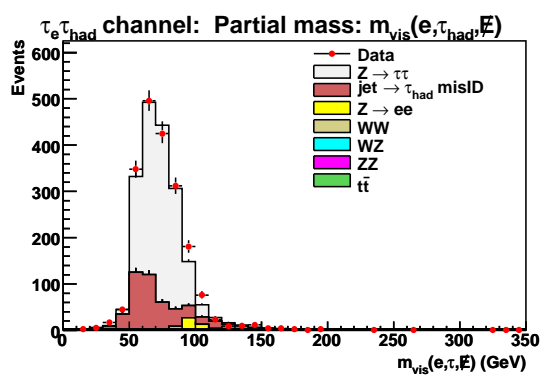

(a) $\tau_{e} \tau_{h a d}$

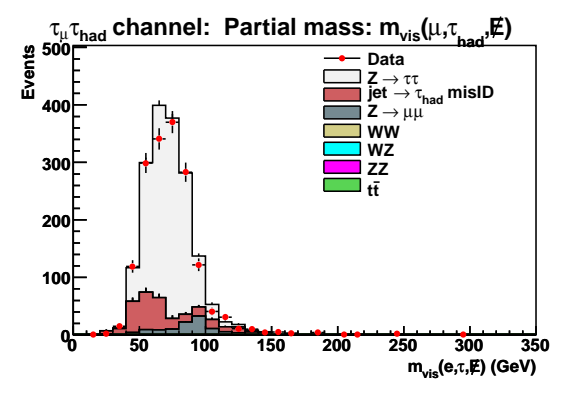

(b) $\tau_{\mu} \tau_{\text {had }}$

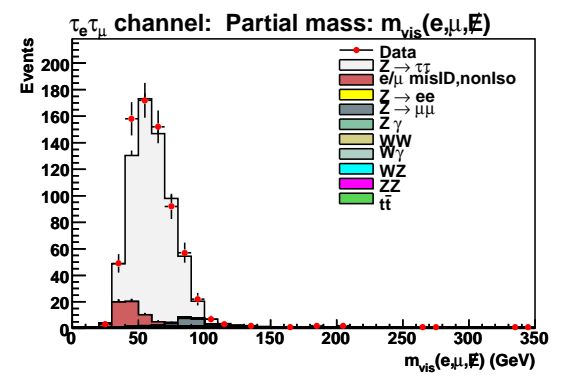

(c) $\tau_{e} \tau_{\mu}$

Figure 8.8: Distributions in the three channels used for signal extraction. All background normalizations are absolute. 
The sources of uncertainty that affect this search could be split in several classes: trigger efficiency, particle identification efficiency scale factors, event selection, background estimation deficiencies, PDFs used in MC generation and luminosity measurement. Most of them affect only the total number of events, but as summarized on Table 8.2, some of them also have impact on the shapes. The fact that templates shifted to account for the effect of shape systematics could contain a different total number of events incorporates the systematics associated to event cuts in a very comfortable way.

The different trigger paths used to collect the data in our final sample require two different objects. The efficiency for each object has been studied deeply and the overall efficiency is assumed to be multiplicative of the two factors. The trigger decision is staggered in three levels, and the efficiencies for each level is calculated separately. In order to make them multiplicative, the calculation is always done with respect to objects that passed the previous level.

The trigger efficiency uncertainty has been calculated to be of a $1 \%$ for muons, a $0.3 \%$ for electrons and a $3 \%$ for hadronic taus. These errors are mostly realted to the limitations in samples used to calculate the efficiency. In the case of hadronic taus, there is no unbiased pure sample of taus ready for a measurement of the trigger efficiency. Therefore, the calculation had to be done with jets, instead of hadronic taus. Given that the identification and selection cuts are above the turn-on of the trigger efficiencies, these systematic uncertainties affect the rates but does not affect the shapes.

Signal detection efficiency is estimated with simulation for most background sources except for jets faking taus and non-isolated leptons. Monte Carlo techniques are used to predict the particle interactions in the detector. We account for possible differences between data and MC particle ID efficiencies through the introduction of scaling factors applied to MC. The uncertainties in determining these factors are assigned as systematic errors of particle reconstruction and ID efficiency. For muons and electrons we use the standard ID's (up to isolation) and assign the corresponding official uncertainties for intermediate- and high- $p_{T}\left(E_{T}\right)$ muons (electrons). In addition we add in quadrature the uncertainty due to the track isolation cuts that are not part of the results in these references, which was found to be very small and independent of the lepton $p_{T}$. These are treated as rate uncertainties 
that do not affect the shapes. For the case of electrons and muons the uncertainty in the data/MC scale factor are different below and above $20 \mathrm{GeV}$. We use common systematic uncertainties obtained by weighting the fraction of electrons (or muons) in the low- and high- $p_{T}$ regions. For a conservative estimate we use the fractions found in the $\tau_{e} \tau_{\mu}$ channel, which contain the larger portion of soft leptons. The fraction of muons below (above) $20 \mathrm{GeV}$ is $42 \%$ (58\%). For electrons, the fractions below (above) $20 \mathrm{GeV}$ are $43 \%$ (57\%), similar to the electrons. We take $1 \%$ uncertainty for muons with $p_{\mathrm{T}}>20 \mathrm{GeV}$, and $4 \%$ uncertainty for muons with $p_{T}<20 \mathrm{GeV}$, and assign overall $2.7 \%$ uncertainty to muon reconstruction and identification. For electrons, we use uncertainties of $0.6 \%(3.2 \%)$ for the case when electron $E_{\mathrm{T}}$ is above (below) $20 \mathrm{GeV}$. and assign uncertainty for electron identification of $2.4 \%$.

Deficiencies in the simulation of calorimeter response to jets lead to MC/data differences in measured $E_{\mathrm{T}}$. These are accounted for by the MC-specific jet energy corrections. To estimate the systematic uncertainties, we vary the jet corrections applied to the MC by one sigma to produce "shifted" templates for the signal extraction/limit setting. This is an example of a shape uncertainty accompanied by some change in the event rate coming through the application of the event cuts.

Shape systematic uncertainties are associated not only to the jet energy scale, but also to the electromagnetic scale and to the tau energy scale. A $1 \%$ uncertainty per electron is used to produce the EM shifted templates. Also, for the tau energy scale, a $1 \%$ uncertainty is applied per tau, due to the limitation of the method.

The systematics related to background estimation from the MC samples are determined by the uncertainties in the cross-sections and branching fractions.

QCD-like backgrounds is estimated with fakes, as previously stated. For nonisolated leptons or leptons inside jets. The statistical error is added in quadrature to the error on the normalization scale factor (same-signs to sidebands). This systematic uncertainty affects the QCD background normalization in the $\tau_{e} \tau_{\mu}$ channel.

For the $\tau_{e} \tau_{\text {had }}$ and $\tau_{\mu} \tau_{\text {had }}$ channels, the estimation of the $j e t \rightarrow \tau$ misidentification is assigned a systematic uncertainty on the normalization of a $15 \%$. This uncertainty is the maximum half difference between the prediction using Fake Rates 


\begin{tabular}{lccc}
\hline \hline Parameter & type & Error $(\%)$ & applies to: \\
\hline \hline$e$ ID & rate & 2.4 & $e$ in MC \\
muon reco+ID & rate & 2.6 & $\mu$ in MC \\
tau ID & rate & 3.0 & $\tau$ in MC \\
$e$ trig & rate & 0.3 & $e$ in MC \\
muon trig & rate & 1.0 & $\mu$ in MC \\
tau trig & rate & 3.0 & $\tau$ in MC \\
\hline z-vertex cut & rate & 0.5 & all MC \\
\hline fake/non-iso lepton bg in $\tau_{e} \tau_{\mu}$ & rate & 20.0 & fake/non-iso bg \\
fake $\tau_{\text {had }}$ in $\tau_{e} \tau_{\text {had }}$ & rate & 15.0 & fake tau bg \\
fake $\tau_{\text {had }}$ in $\tau_{\mu} \tau_{h a d}$ & rate & 15.0 & fake tau bg \\
$\sigma \times \mathcal{B}(Z \rightarrow l l)$ & rate & 2.2 & $Z$ MC \\
$Z \rightarrow l l$ backgrounds & rate & 6 & $\tau_{e} / \mu \tau_{h a d}$ \\
$\sigma(t \bar{t})$ & rate & 13.4 & $t \bar{t} \mathrm{MC}$ \\
di-boson cross sections & rate & 10 & di-boson MC \\
PDFs $(H i g g s)$ & rate & 5.7 & signal \\
Luminosity & rate & 6.0 & all MC \\
\hline JES & shape & $\pm 1 \sigma($ per jet $)$ & all MC \\
EM scale & shape & \pm 1.0 & $e$ in MC \\
Tau $p_{T}$ scale & shape & \pm 1.0 & $\tau_{\text {had }}$ in MC \\
\hline \hline
\end{tabular}

Table 8.3: Systematic uncertainties by source.

extracted for only the leading jet and for exclusively the subleading jet in the event.

Because the main production mechanisms of signal events are gluon fusion and b-quark annihilation, the error on the parton distribution functions have a strong impact in the signal acceptance. The total systematic uncertainty on signal acceptance related to the limited knowledge of the PDFs is a $5.7 \%$.

The luminosity uncertainty in CDF has been estimated to be a $6 \%$, due to the limitations of the modeling of the simulation and performance of the Cerenkov counters. Table 8.2 summarizes the systematic uncertainties. 


\subsection{Fitting Method}

An eventual Higgs signal like the one searched in an analysis like this would lay on top of a very large background from $Z / \gamma * \rightarrow \tau \tau$, plus other smaller backgrounds including fakes and diboson production. Instead of only counting events, a technique based on fitting templates adds sensitivity because it it uses the information stored on the shapes. To distinguish the Higgs signal from this background we use the "visible mass" variable, $m_{v i s}$, defined as the mass of a pseudo-four-vector formed from adding together the lepton and reconstructed tau four vectors to the transverse and total energy components of the $\mathscr{E}_{\mathrm{T}}$ vector (setting its $z$ component to zero):

$$
m_{v i s}=\sqrt{\left(\sum_{\phi} E_{\phi}\right)^{2}-\left(\sum_{\phi} \boldsymbol{p}_{\phi}\right)^{2}}
$$

where $\phi$ refers to $\tau_{1}, \tau_{2}$ and $E_{\mathrm{T}}$, and

$$
\boldsymbol{E}_{\mathbf{T}}=\left(\begin{array}{llll}
E_{\mathrm{T}}^{x}, & E_{\mathrm{T}}^{y}, & 0, & E_{\mathrm{T}}
\end{array}\right)
$$

This variable provides better signal discrimination against background than other ones, like transverse mass. The fact that the variable has or doesn't have a physical meaning is not relevant, as long as the shapes are under control and shows discriminant power. The distribution shape of this variable for samples of signal and background is shown in Figure 8.9, only $\tau_{l} \tau_{\text {had }}$ channel.

The likelihood function is built with the $m_{v i s}$ spectrum:

$$
\mathcal{L}=\prod_{i j} \frac{\mu_{i j}^{n_{i j}} e^{-\mu_{i j}}}{n_{i j} !}
$$

where $n_{i j}$ is the observed number of events in the $m_{v i s}$ distribution, bin $i$, for channel $j$ (where $j=1$ corresponds to the $\tau_{e} \tau_{h}$ channel, $j=2$ to $\tau_{\mu} \tau_{h}$, and $j=3$ to $\tau_{e} \tau_{\mu}$ ), and $\mu_{i j}$ is the expected number of events in that bin. 


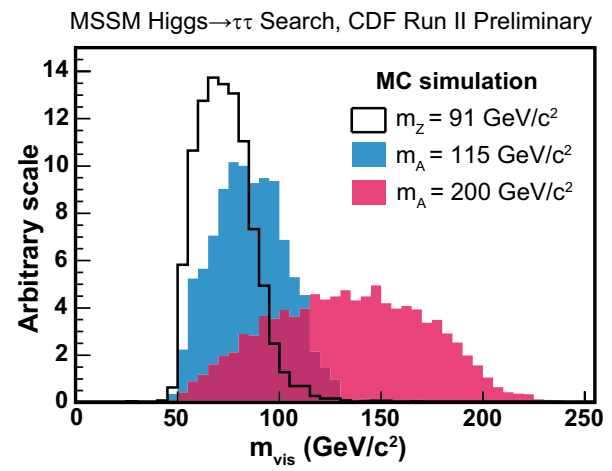

Figure 8.9: Visible mass distributions for $Z$ and Higgs bosons (normalized to the same area).

The total expected number of events is the combination of all the different sources (backgrounds and signal, index $k$ ). The spectrum of each source is normalized with the Luminosity $L$, the cross section times branching ratio $\sigma_{k}$ for source $k$, and the detection efficiency in the bin $\epsilon_{i j k}$ :

$$
\mu_{i j}=\sum_{k} L \sigma_{k} \epsilon_{i j k}
$$

In principle if all the parameters except the signal cross section $\sigma_{h}$ were known exactly, we could apply Bayes' Theorem to arrive at a posterior density $\mathcal{P}\left(\sigma_{h}\right)$ in the unknown variable:

$$
\mathcal{P}\left(\sigma_{h}\right)=\frac{\mathcal{L}\left(\sigma_{h}\right)}{\int_{0}^{\sigma_{\max }} \mathcal{L}\left(\sigma_{h}^{\prime}\right) d \sigma_{h}^{\prime}}
$$

Here we assume a uniform prior density in the unknown signal cross section up to some maximum cutoff. This posterior density $\mathcal{P}\left(\sigma_{h}\right)$ can then be used to determine confidence intervals and estimate the true value of the signal rate. 


\subsection{Nuisance Parameters for Systematic Uncertain- ties}

The systematic uncertainties referred to in Section 8.2 are incorporated as nuisance parameters in the likelihood, see Table 8.2. Each of these uncertainties affect some or all the background or signal sources in some or or all the channels. The uncertainty on the luminosity affects the normalization of all the backgrounds and all channels, for instance. However the electron ID scale factor uncertainty does not impact the $\tau_{\mu} \tau_{h}$ channel. Also, the $Z / \gamma^{*} \rightarrow \tau \tau$ cross section assumed is taken from the CDF measured value of $254.9 \pm 5.7 \mathrm{pb}$ (excluding the luminosity uncertainty) and affects only some backgrounds in all he channels. The systematic uncertainties incorporated in the fit are

- integrated luminosity,

- background cross sections,

- QCD ( $\tau$ fake) rates,

- lepton and tau trigger/ID data/MC ratios,

- PDFs, and

- electron, tau and jet energy scale.

Each of these systematic uncertainties are related to a parameter in the likelihood, and these parameters are allowed to float in the fit. However, the parameters are not completely free, but are constrained to the nominal value with a gaussian corresponding to the uncertainty in the parameter. The signal cross section does not fluctuate, it is the parameter used for the scan. The overall likelihood can then be written

$$
\mathcal{L}=\prod_{i j} \frac{\mu_{i j}^{n_{i j}} e^{-\mu_{i j}}}{n_{i j} !} \times G\left(L, L_{0}, \sigma_{L}\right) \times \ldots
$$

where the $G$ functions are gaussians constraining the values of the imprecisely known parameters. 


\subsubsection{Template Morphing}

The energy scale uncertainties are treated using a "template morphing" technique. Each background source bin efficiency $\epsilon_{i j k}$ is calculated for the nominal case, and for $\pm 1-\sigma$ shifts due to the uncertainty in the electron, tau, and jet scale uncertainty. Then, a morphing parameter controls the admixture of the nominal and shifted bin efficiency when calculating the expected number of events in a bin. For example, let $f^{e}$ represent the electron energy scale morphing parameter, then, the expected number of events in a given bin $i j k$ can be written in terms of an efficiency as

$$
\epsilon_{i j k}=\epsilon_{i j k}^{n o m}+f^{e}\left(\frac{\epsilon_{i j k}^{+}-\epsilon_{i j k}^{-}}{2}\right) .
$$

Thus the nominal value of the number of expected events corresponds to $f^{e}=0$, and a unit gaussian constraint term on the value of $f^{e}$ is added to the likelihood. The tau and jet energy scale uncertainties are handled in similar fashion simultaneously. The predicted number of events from a given source in a bin is never allowed to be negative as a result of the morphing.

\subsubsection{Profile Likelihood}

The nuisance parameters in the likelihood need to disappear before a posterior density in the signal rate $\mathcal{P}\left(\sigma_{h}\right)$ can be obtained. The pure Bayesian method would proceed with a marginalization by integrating the likelihood over the nuisance parameters. A different method, referred to as profiling, has been chosen for this analysis. In this method, the likelihood is maximized with respect to all the nuisance parameters (using MINUIT) at each point in $\sigma_{h}$. This became the preferred choice because the profile calculation is more than an order of magnitude faster to compute and gives results nearly identical to the marginalization calculation. The resulting profile likelihood $\mathcal{L}^{\max }\left(\sigma_{h}\right)$ is used exactly as described above in deriving the posterior density $\mathcal{P}\left(\sigma_{h}\right)$.

Given that a flat prior with a cut-off was chosen, the normalization from likelihood to obtain probability is trivial. Therefore, to obtain a 95\% CL limit on the value 
of $\sigma_{h}$, the posterior density has to be integrated to that value of $\sigma_{h}$ below which $95 \%$ of the probability density lies. Even when a signal excess is observed, one could follow this procedure to calculate a meaningful exclusion limit.

\subsection{Expected Sensitivity}

The expected sensitivity of the search is assessed by performing many pseudoexperiments. In each of these, we generate an outcome based on the expected number of events in each bin from a random Poisson distribution about the mean number expected. Given that nothing is known about the potential signal generated by new physics, zero signal cross section is assumed. At this stage, the primary goal of this search is setting limits.

For each pseudo-experiment, the 95\% CL upper limit is calculated as discussed in the previous section. A distribution of expected 95\% CL upper limits is formed for each mass point. The median of the distributions are taken as the expected limit, and 1 and 2- $\sigma$ points integrate, respectively $68 \%$ and $95 \%$ of the pseudoexperiments. Table 8.4 shows the median expected upper limit, and also the $\pm 1 \sigma$ and $\pm 2 \sigma$ ranges.

\subsection{Results}

A fit to observed data is shown in Figure 8.11. The different templates are normalized and shifted to the fit output and a signal contribution for a Higgs of $\mathrm{m}_{A}$ has been normalized to the $95 \%$ CL exclusion limit.

The last column in Table 8.4 shows the observed 95\% CL upper limit for each assumed Higgs mass. They are in agreement with the expectation and there is no evidence for Higgs signal. The same information is displayed in Figure 8.10. 


\begin{tabular}{|c|ccccc|c|}
\hline \hline $\begin{array}{c}\text { mass } \\
(\mathrm{GeV})\end{array}$ & $-2 \sigma$ & $-1 \sigma$ & $\begin{array}{c}\text { median } \\
(\mathrm{pb})\end{array}$ & $+1 \sigma$ & $+2 \sigma$ & $\begin{array}{c}\text { observed } \\
(\mathrm{pb})\end{array}$ \\
\hline 90 & 18.576 & 23.422 & 29.051 & 35.129 & 42.122 & 27.651 \\
100 & 10.322 & 13.278 & 17.445 & 23.543 & 31.834 & 24.805 \\
110 & 4.251 & 5.672 & 7.943 & 11.209 & 15.174 & 11.528 \\
120 & 2.469 & 3.248 & 4.623 & 6.689 & 8.966 & 5.873 \\
130 & 1.711 & 2.314 & 3.080 & 4.250 & 5.706 & 3.214 \\
140 & 1.189 & 1.596 & 2.191 & 3.028 & 4.095 & 2.101 \\
150 & 0.907 & 1.172 & 1.648 & 2.355 & 3.119 & 1.596 \\
160 & 0.739 & 0.991 & 1.334 & 1.822 & 2.476 & 1.361 \\
170 & 0.563 & 0.787 & 1.062 & 1.537 & 2.083 & 1.179 \\
180 & 0.486 & 0.655 & 0.903 & 1.259 & 1.785 & 0.977 \\
190 & 0.430 & 0.562 & 0.793 & 1.097 & 1.538 & 0.885 \\
200 & 0.363 & 0.483 & 0.672 & 0.955 & 1.260 & 0.768 \\
230 & 0.261 & 0.338 & 0.482 & 0.672 & 0.961 & 0.532 \\
250 & 0.213 & 0.281 & 0.379 & 0.519 & 0.718 & 0.465 \\
\hline \hline
\end{tabular}

Table 8.4: Expected and observed 95\% CL limits on the Higgs signal cross section. For the expected limits, the columns illustrate the range of the expectation around the median. 


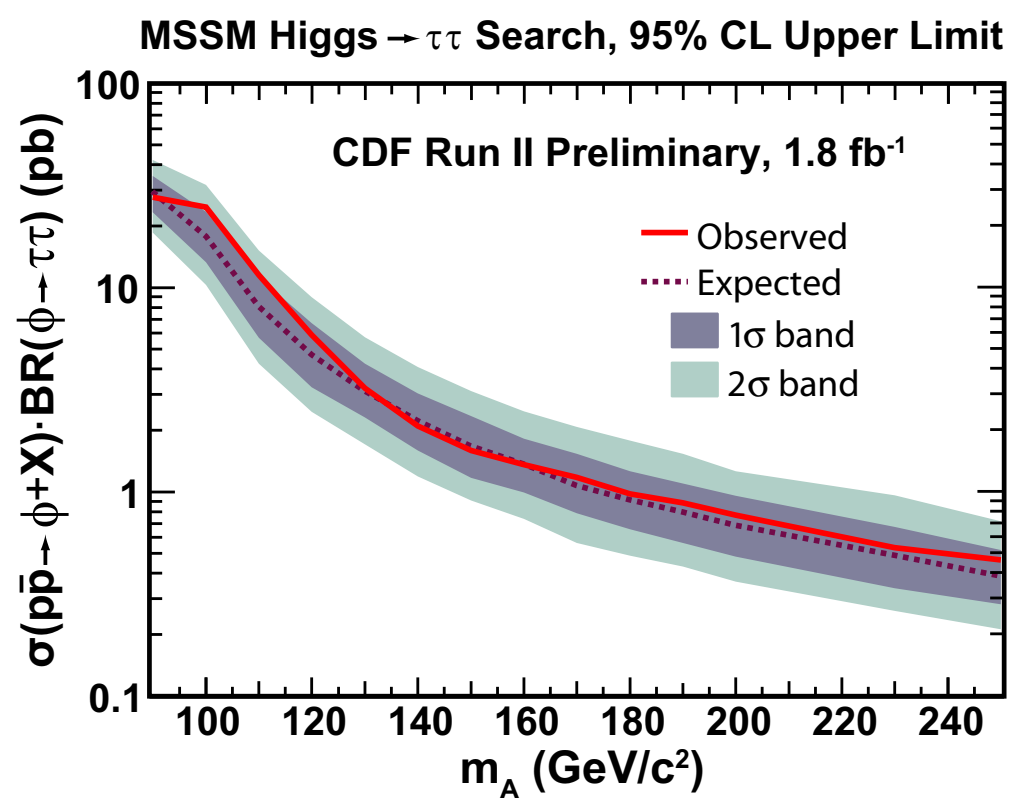

Figure 8.10: Observed and expected 95\% CL upper limits on $\sigma(p \bar{p} \rightarrow \phi+X) \times$ $B R(\phi \rightarrow \tau \tau)$ as a function of Higgs mass. The shaded areas show the $\pm 1,2 \sigma$ bands on the expected limits. 


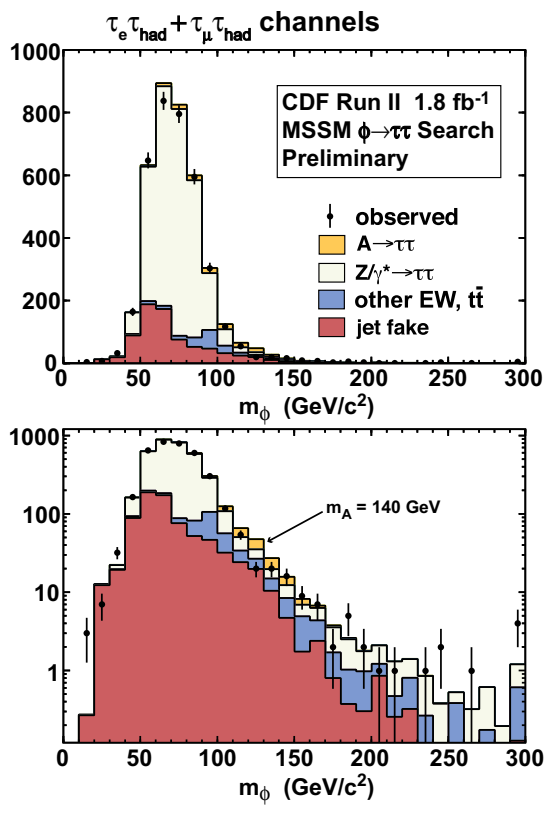

(a) $\tau_{e} \tau_{h a d}+\tau_{e} \tau_{h a d}$
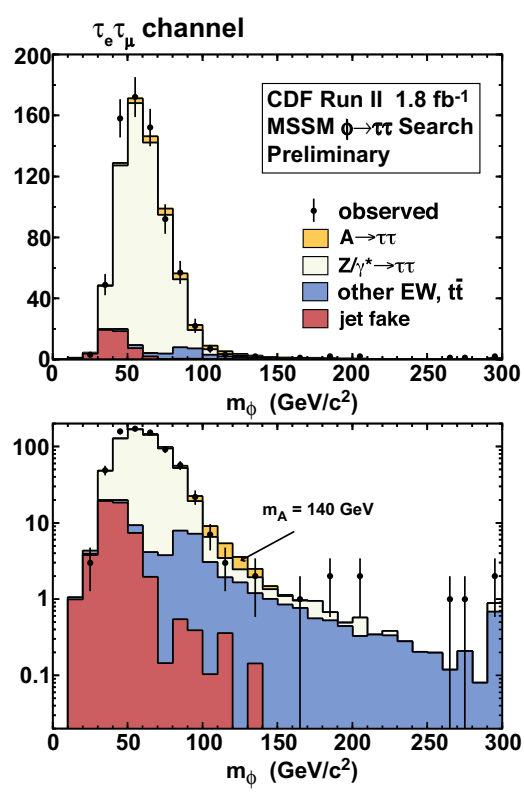

(b) $\tau_{e} \tau_{\mu}$

Figure 8.11: Observed distribution of $m_{v i s}$ along with a signal corresponding to $m_{A}=140 \mathrm{GeV}$. The signal distribution is normalized to the excluded signal at $95 \%$ CL. The backgrounds are normalized to the expectations after performing the fits. 


\subsection{Interpretation of the Limits}

The observed limits $@ 95 \%$ CL on $\sigma(p \bar{p} \rightarrow \phi) \times \mathcal{B}(\phi \rightarrow \tau \tau)$ are used to exclude regions in the $\tan \beta$ vs $m_{A}$ plane, where $\tan \beta$ is the ratio of the vacuum expectation values for the Higgs fields that couples to the up- and down-type fermions; $m_{A}$ is the mass of the $C P$-odd neutral Higgs boson.

The MSSM parameter space is quite large. For the interpretation, we take two benchmarks [102] that have become the standard in the field - the $m_{h}^{\max }$ and the no-mixing scenarios.

The $m_{h}^{\max }$ scenario has parameters chosen such that the maximum possible Higgs mass as a function of $\tan \beta$ is obtained. The no-mixing scenarios additionally assumes no mixing in the stop-squark sector. The common SUSY parameters for these benchmarks are Higgs mixing parameter $\mu=+0.2 \mathrm{TeV}, S U(2)$ gaugino mass parameter $M_{2}=0.2 \mathrm{TeV}$. The difference is in the choice of the SUSY mass scale $M_{S U S Y}$, the squark mixing parameter $X_{t}$, and gluino mass $m_{\tilde{g}}$. For $m_{h}^{\max }$, $M_{S U S Y}=1 \mathrm{TeV}, X_{t}^{\overline{M S}}=\sqrt{6} M_{S U S Y}$. The corresponding values for no-mixing are $M_{S U S Y}=2 \mathrm{TeV}$ and $X_{t}=0$. In both cases $m_{\tilde{g}}=0.8 M_{S U S Y}$. In all calculations we used top quark mass $m_{t}=178.0 \mathrm{GeV} / \mathrm{c}^{2}$ (using $m_{t}=174.0 \mathrm{GeV} / \mathrm{c}^{2}$ has negligible effect on the results). Earlier benchmarks [103] have the same parameters except for $\mu=-0.2 \mathrm{TeV}$ and $M_{S U S Y}=1 \mathrm{TeV}$ for both the $m_{h}^{\max }$ and the no-mixing scenarios

As discussed in Chapter 2, the MSSM neutral Higgs sector has three bosons, the pseudoscalar $A$, and the scalar $h$ and $H$. One of the scalars (either $h$ or $H$ ) is nearly degenerate in mass and production cross-section with the $A$. For a given value of $\tan \beta$ in a given scenario, there is a "crossover mass" below which the $h$ shadows the $A$ and above which it is the $H$. In the $m_{h}^{\max }$ scenario the crossover point is at $\mathrm{m}=130 \mathrm{GeV}$. 


\subsubsection{Production Cross-section Calculations}

There are two production modes that are relevant for our search. Gluon fusion, $g g \rightarrow \phi$, and $b \bar{b}$ annihilation, $b \bar{b} \rightarrow \phi$, where $\phi$ is $A, H, h$.

The full MSSM production cross-sections for $b \bar{b} \rightarrow \phi$ has not been calculated. However, this process has been calculated to NLO and NNLO for the Standard Model Higgs [104] [105]. From the calculated cross sections for $b \bar{b} \rightarrow \phi$ in the Standard Model, we could naively apply a factor of $\tan ^{2} \beta$. However, this would not take into account radiative effects. To do this properly we use the FeynHiggs program by S. Heinemeyer [106]. This program takes MSSM parameters as input and outputs the couplings and branching ratios for the SM and MSSM Higgses. We take the ratio $\Gamma_{\phi \rightarrow b b}^{\mathrm{MSSM}} / \Gamma_{\phi \rightarrow b b}^{\mathrm{SM}}$ and multiply the SM production cross section of Kilgore et al. to get the $b \bar{b} \rightarrow \phi$ production cross section in the MSSM. Here $\Gamma_{\phi \rightarrow b b}^{\mathrm{MSSM}}, \Gamma_{\phi \rightarrow b b}^{\mathrm{SM}}$ are the partial widths of $\phi \rightarrow b \bar{b}$ in MSSM and SM calculated by FeynHiggs. The $\Delta m_{b}$ correction to $\tan \beta$ changes the coupling of the Higgs to b, and therefore to $\tau$ as well. We cannot ignore the effect of $\Delta m_{b}$ on the branching ratio, since as the coupling of the Higgs to b goes down (up), the corresponding branching fraction to $\tau \tau$ goes up (down). Therefore, we use the values of Higgs to $\tau \tau$ branching ratio output by the FeynHiggs program.

For the cross-sections for gluon fusion we use the HIGLU program by M. Spira [107].A somewhat dated manual can be found at [108]. It calculates the NLO cross-sections for $g g \rightarrow A, H, h$ (using CTEQ6 PDFs). The various MSSM parameters such as $\tan \beta, \mu, M_{S U S Y}$, and the tri-linear couplings are taken as input parameters. HIGLU can calculate the cross-sections for any $\tan \beta$ but does not take into account radiative corrections modifying the Higgs couplings to $b$ quarks have a significant effect. Fortunately, these corrections behave exactly like in the case of $b \bar{b} \rightarrow$ Higgs [109]. Therefore, we calculate the SM cross-section using HIGLU and apply the MSSM enhancement factors from FeynHiggs as described above for the $b \bar{b} \rightarrow$ Higgs case. 


\subsubsection{Excluded region in $\tan \beta$ vs $m_{A}$}

Figure 8.12 shows the excluded parameter region in the $\tan \beta$ vs $m_{A}$ plane. As a result of the observed excess of events, the observed $\tan \beta$ exclusions are significantly weaker than the expectation.

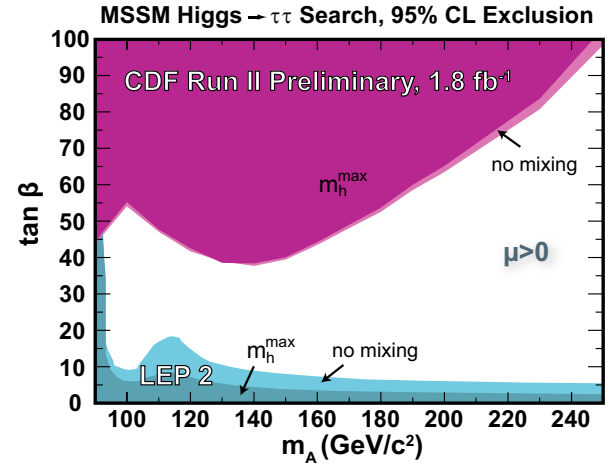

(a) $\mu>0$

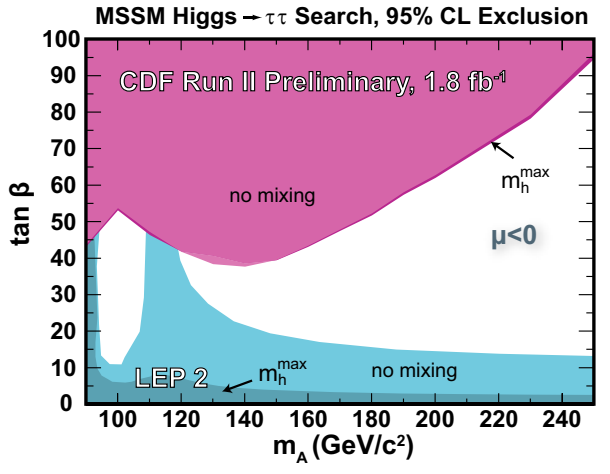

(b) $\mu<0$

Figure 8.12: Excluded $\tan \beta$ as a function of $m_{A}$ for the "no-mixing" and $m_{h}^{\max }$ scenarios with $\mu>0$ and $\mu<0$.

\subsection{Full di-tau mass reconstruction using MET pro- jection.}

Full di-tau mass reconstruction is possible in cases when the parent particle $(Z$, $\phi)$ has substantial boost in the transverse plane (tau decay products are not backto-back). The reconstruction method relies on the "collinear approximation": the direction of the neutrinos from tau decays is taken to be the same as the one of the visible decay products. This is a reasonable assumption for fairly energetic taus ${ }^{1}$ Using $E_{\mathrm{T}}$ to represent the sum of the transverse momenta of the neutrinos, one can determine their energies $E_{\nu}^{(i)}$ by solving the system of equations:

\footnotetext{
${ }^{1}$ Obviously this approximation works better for hadronic tau decays. In leptonic decays there are two neutrinos, leading to an additional approximation.
} 


$$
\begin{aligned}
& E_{\nu}^{(1)} \sin \theta^{(1)} \cos \phi^{(1)}+E_{\nu}^{(2)} \sin \theta^{(2)} \cos \phi^{(2)}=E_{x}^{\text {miss }} \\
& E_{\nu}^{(1)} \sin \theta^{(1)} \sin \phi^{(1)}+E_{\nu}^{(2)} \sin \theta^{(2)} \sin \phi^{(2)}=E_{y}^{\text {miss }}
\end{aligned}
$$

Here $\theta^{(i)}, \phi^{(i)}$ are the polar and azimuthal angles of the visible tau decay products, $E_{x}^{\text {miss }}$ and $E_{y}^{\text {miss }}$ are the two components of the corrected $E_{\mathrm{T}}$. The neutrino energies are added to the measured energies of the visible decay products, allowing the reconstruction of the full di-tau mass. Events with negative energy solutions are discarded (some of them can be recovered if we take into account $E_{\mathrm{T}}$ resolution).

This method is very promising for LHC, where the statistics are not an issue. It was applied at CDF in Run I [110] in the $\tau_{e} \tau_{\text {had }}$ channel but due to limited statistics the sensitivity of extracting information from the mass distribution was shown to be weaker than performing a counting experiment.

In the following plots the selected non back-to-back events must satisfy $\mid \sin \left(\phi^{(1)}-\right.$ $\left.\phi^{(2)}\right) \mid>0.3$, which was found to be a good compromise between resolution and efficiency. After some tuning, non back-to-back events will be treated as separate channels in the next update of the results. The plots in Figure 8.13 are shown to demonstrate the current performance of full di-tau mass reconstruction. 


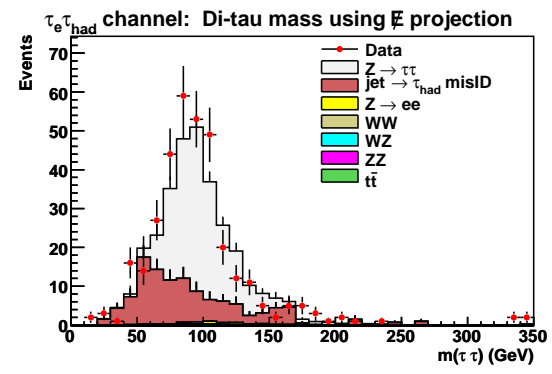

(a) $\tau_{e} \tau_{h a d}$

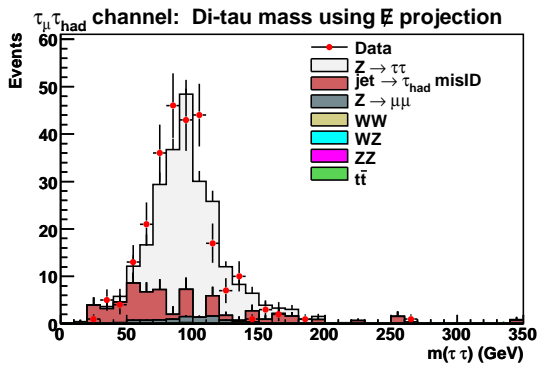

(b) $\tau_{\mu} \tau_{h a d}$

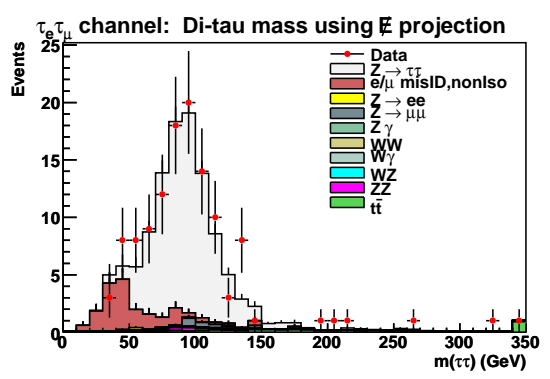

(c) $\tau_{e} \tau_{\mu}$

Figure 8.13: Di-tau mass reconstructed with the $\mathcal{E}_{\mathrm{T}}^{\prime}$ projection method. 


\section{Chapter 9}

\section{Conclusions}

A search for for neutral MSSM Higgs bosons in the di- $\tau$ decay channel has been performed with $1.8 \mathrm{fb}^{-1}$ of data. Given that no evidence for signal over the expected SM backgrounds has been found, 95\% CL exclusion limits have been set on the production cross sections times branching fraction to taus. The signal extraction techniques used in this analysis have proven to improve the sensitivity.

The sensitivity achieved by this analysis is based on two points: the robustness of the description of the different backgrounds and the boldness of the method for tau reconstruction.

As stated in the introductory sections, despite its smaller branching fraction, the $\tau \tau$ decay channel provides much better sensitivity when compared to other decay channels. Still unpublished, but already authorized by the CDF collaboration, the latest release of the search for MSSM Higgs bosons in the 3b channel shows an improving, but still poorer, sensitivity, Figure 9.1

The only experiment capable of performing comparable searches is DØ. Again, the sensitivity of this analysis provides a larger excluded region, due partially to the larger dataset used, Figure 9.2

The fact that this result has been included in the Particle Data Group Review of Particle Physics [4] shows not only the relevance of analysis in the field, but 


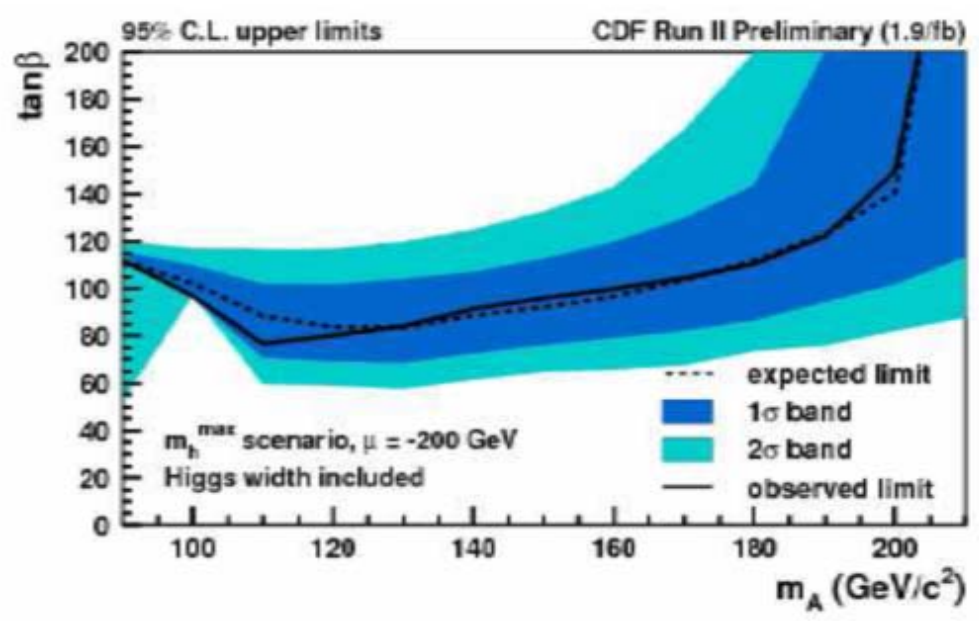

Figure 9.1: Excluded $\tan \beta$ as a function of $m_{A}$ for the 3 b-quark analysis at $\mathrm{CDF}$

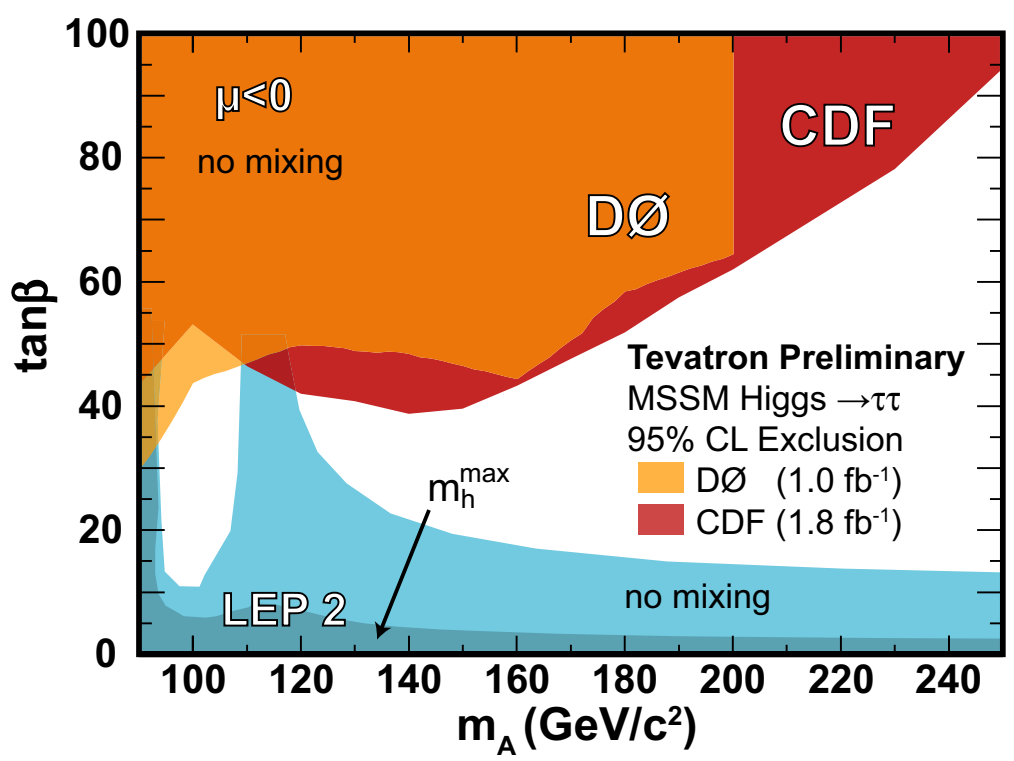

Figure 9.2: Excluded $\tan \beta$ as a function of $m_{A}$ regions for LEP, D $\varnothing$ and CDF using the $\tau \tau$ decay channel 
also the endorsement of the community. Nevertheless, several improvements could be implemented to further increase the sensitivity. These include the application of multivariate techniques or splitting this inclusive search into groups depending on the presence of b-tagged jets. However, the biggest improvement is coming in a few months, with the beginning of an LHC program that promises to finally conquer the Terascale. 


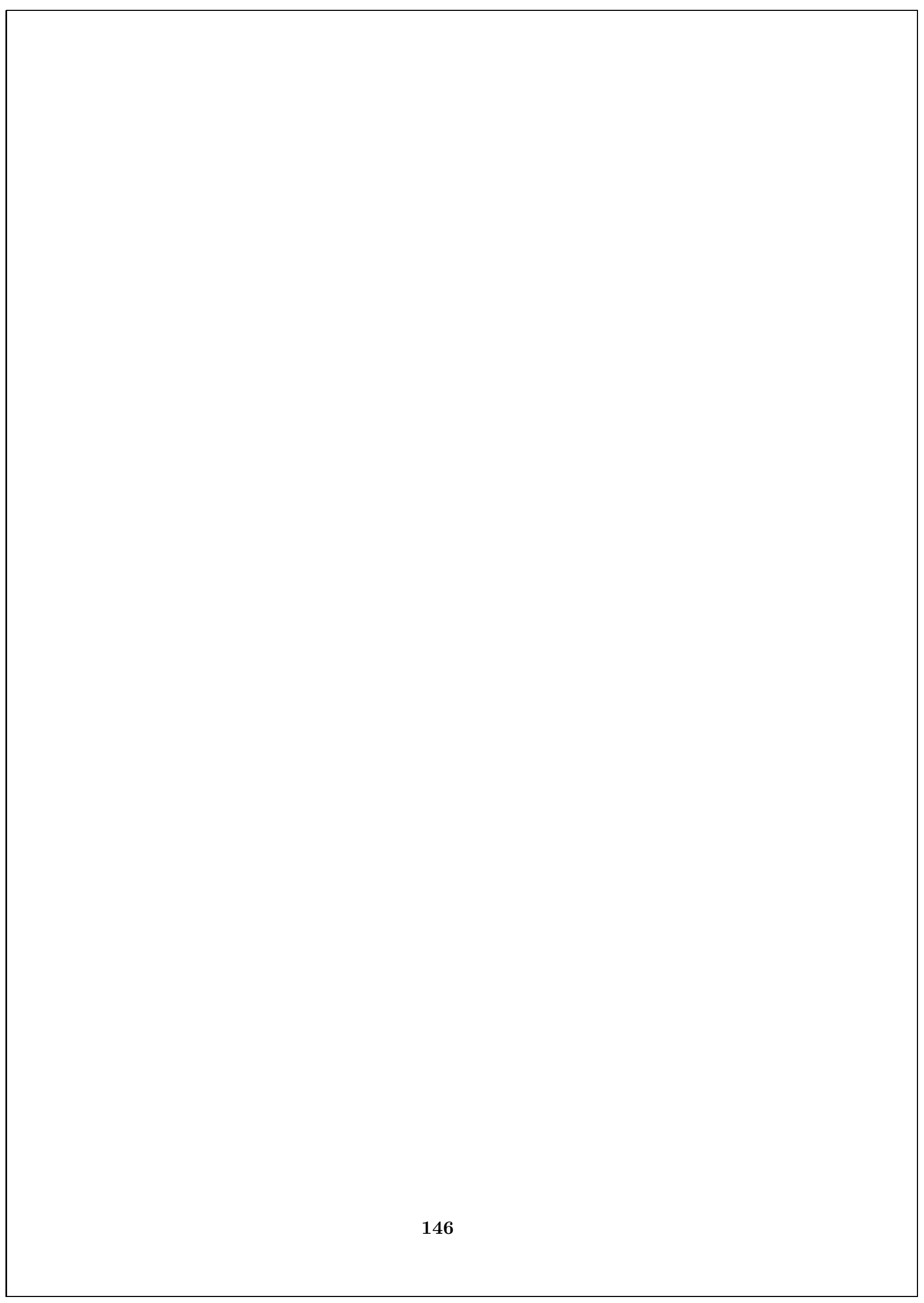




\section{Appendix A}

\section{Resum}

Aquest treball exposa els resultats obtinguts en la cerca dels bosons de Higgs neutres en el context del la Mínima Extensió Superesimètrica del Model Estàndard. L'anàlisi descrit a la tesi ha estat realitzat amb les dades preses pel detector CDF, al col.lisionador hadrònic Tevatron, Fermilab (EUA).

\section{A.1 Introducció teòrica}

El Model Estàndard, MS, descriu la natura a partir de dos tipus fonamentals de partícules, els bosons i els fermions. Per una banda, els fermions constintueixen la matèria i són sis anomenats leptons i sis quarks. Aquestes partícules responen a l'estadística proposada per Fermi i Dirac i estan organitzats en una estructura de tres famílies. Per una altra banda, els bosons segueixen l'estadística proposada per Bose i Enstein, son les partícules responsables de les interaccions entre els fermions, taula 2.1. El MS incorpora sota el seu formalisme les interaccions electrofeble i forta.

L'electró (e), el muó $(\mu)$, el tau $(\tau)$, els corresponents neutrins $\left(\nu_{\mathrm{e}}, \nu_{\mu}, \nu_{\tau}\right)$ juntament amb les seues antipartícules són els leptons. Els quarks s'anomenen up (u), down (d), strange (s), charm (c), bottom (b) i top (t), i cadascun d'ells té un company 
d'antimatèria. La interacció electromagnètica està mediada pel fotó $(\gamma)$, que és un dels bosons del MS; la interacció forta està mediada pels gluons, g, i la feble pels bosons $\mathrm{Z} \mathrm{i} \mathrm{W}^{ \pm}$.

El MS és una teoria quàntica de camps galga, en la qual les interaccions fonamentals es deriven de les simetries imposades al lagrangià. L'excel.lència en l'acord entre el model teòric i l'experiment no té precedent en la història de la ciència. Tanmateix, alguns aspectes de la teoria, com ara el mecanisme de Higgs per al trencament de la simetria electrofeble, encara no han pogut comprobar-se experimentalment. Aquesta i algunes altres dificultats teòriques i experimentals suggereixen que, més que una teoria del tot, el MS és més un model efectiu a baixa energia.

La supersimetria és un dels candidats a superar les dificultats del MS. Afegint una nova simetria que associa un fermió a cada bosó i viceversa, aquesta nova teoria resol els problemes del MS. La Mínima Extensió Supersimètrica del Model Estàndard, MSMS, és la concreció de la supersimetria que exigeix una menor ampliació del sector Higgs del MS.

Mentre que el MS proposa la introducció d'un doblet Higgs, el MSMS n'introdueix dos. Els graus de llibertat adicionals incorporats a la teoria materialitzen en cinc nous estats físics. És a dir, si al MS només hi havia un bosó de Higgs, en MSMS hi apareixen cinc, normalment representats com $\mathrm{A}^{0}, \mathrm{H}^{0}, \mathrm{~h}^{0} \mathrm{i} \mathrm{H}^{ \pm}$. Els tres primers són neutres, mentre que els dos últims són carregats. A nivell arbre, la fenomenologia del sector Higgs es pot parametritzar amb dos paràmetres: $\tan \beta$ i m $\mathrm{A}^{0}$, figura 2.6.

Els processos de producció de bosons de Higgs del MSMS neutres al Tevatron són la fusió de gluons, g $\mathrm{g} \rightarrow \mathrm{A}^{0}$, i l'aniquil.lació de quarks $\mathrm{b}, \mathrm{b} \overline{\mathrm{b}} \rightarrow \mathrm{A}^{0}$. Les seccions eficaces de producció mitjancant aquestos dos processos es pot trobar a la Figura 2.9 (seccions eficaces en pb). Els bosons de Higgs no són estables, sinó que es desintegren immediatament en altres partícules. Les fraccions d'embrancament de les desintegracions dels bosons neutres són practicament constants dintre dels intervals de $\tan \beta \mathrm{i} \mathrm{m}_{\mathrm{A}^{0}}$ d'interés al Tevatron, figura 2.10 .

Encara que el canal fonamental de desintegració és $\mathrm{A}^{0} \rightarrow \mathrm{b} \overline{\mathrm{b}}$, el canal $\mathrm{A}^{0} \rightarrow \tau^{+} \tau^{-}$ ha mostrat millor sensibilitat. Açó es deu a què en un col.lisionador hadrònic, com ara el Tevatron, la producció de parelles de quarks b, b $\bar{b}$, és molts ordres 
de magnitud major a la producció de bosons de Higgs. L'extracció d'una possible senyal provinient del Higgs en un canal com b b és molt més complicada per la magnitud i la dificultat de modelar adequadament aquestos fons. Per aquesta raó, les búsquedes de bosons de Higgs en desintegracions a parelles de quarks b no poden sondejar els mecanismes principals de producció, sinó que s'han de buscar en producció associada (per exemple, a altres quakrs b).

Amb les desintegracions a $\tau^{+} \tau^{-}$, la cerca és sensible als bosons de Higgs produïts amb els dos mecanismes principals. La dificultat de l'anàlisi, tanmateix, rau a identificar eficientment leptons tau i modelar correctament els fons. Els problemes de la reconstrucció de taus provenen del fet que aquest leptó no és estable, sinó que es desintegra ràpidament a altres leptons o a mesons. Les desintegracions hadròniques, que incluen la majoria de la fracció d'embrancament dels taus, tenen una signatura similar a la dels jets, que es produeixen abundantment al Tevatron.

L'estrategia de l'anàlisis presentada en aquesta memòria consisteix en seleccionar successos en els quals un dels taus es desintegra leptònicament, es a dir, $\tau \rightarrow \mathrm{e} \bar{\nu}_{\mathrm{e}} \nu_{\tau}$ o $\tau \rightarrow \mu \bar{\nu}_{\mu} \nu_{\tau}$, i l'altre es desintegra leptònicament, amb un sabor diferent que el primer, o hadrónicament.

\section{A.2 Dispositiu Experimental}

El Tevatron es un col.lisionador de protons i antiprotons, $\mathrm{p} \overline{\mathrm{p}}$, amb una energia al centre de masses de $\sqrt{s}=1.96 \mathrm{TeV}$. Es l'últim pas d'un complexe d'acceleradors situats a Fermilab, als Estats Units d'Amèrica. Aquestes col.lisions són les més energètiques que es produeixen a un laboratori i permeten l'estudi de la física a la frontera del coneixement.

CDF i DØ són els detectors que s'han construït per a analitzar les col.lisions del Tevatron. Aquestos experiments han estat disenyats i possats en funcionament per col.laboracions internacionals formades per centenars de científics.

La cerca que es descriu en aquesta tesi es basa en les dades presses per CDF fins a Març de 2007. Aquest detector té l'estructura habitual dels experiments a la física d'altes energies: una geometria cilíndrica al voltant del feix de partícules en 
la qual diferents subsistemes han estat instal.lats concèntricament, figura 3.5.

El sistema més proper al feix és el detector de traces, format per un detector de sicili i una cambra de deriva. Aquest sistema està dintre un camp magnètic d'1.4 T que corba les trajectòries de les partícules carregades. Aquestes partícules, en atravessar els sensors de sicili creen parells portador-forat que generen corrents que poden ser digitalitzats. Radialment de dins cap a fora, el detector de silici està format pel Layer 00 (L00), SVXII i ISL. Seguint en aquesta direcció, la COT és una cambra de deriva que envolta el silici. Les partícules ionitzen el gas, on un fort camp elèctric separa i atrapa les càrregues. Aquestes càrregues son recollides per un o més fils conductors repartits pel volum de la cambra. Donada la geometria concreta d'aquest detector, es poden reconstruir les trajectòries tri-dimensionals de les partícules que la creuen, figura 3.6.

Al recorregut cap a l'exterior del detector, els calorímetres són els següent subsistema. Un potent imant solenoidal separa el detector de traces dels calorímetres centrals, constituïts per vint-i-quatre falques que conformen un cilindre buit. Adicionalment, els laterals del detector també estan recoberts per una extensió dels calorímetres, figura 3.9.

Finalment, l'espectròmetre de muons de CDF està format per una col.lecció de sistemes amb diferents tecnologies, figura 3.11. En atravessar un muó el detector de muons, deixa una col.lecció de senyals que poden ser agrupats en matrius. Posterioment, es combinen aquestes matrius amb les trajectòries del detector de traces per a obtenir més precisió i puresa a la reconstrucció.

No totes les col.lisions produïdes pel Tevatron són interessants des del punt de vista de la física d'altes energies. Donada la limitació de tecnologia actual per a emmagatzemar els successos, cal dissenyar un sistema de dispar que seleccione en línia les col.lisions relevants per a fer els estudis objecte de d'aquestos experiements. Aquest sistema, anomenat trigger, té a CDF una arquitectura basada en tres nivells de selecció, figura 3.13. L'avaluació de l'eficiència del sistema de trigger és una part fonamental de qualsevol anàlisi. 


\section{A.3 Mostra de dades $\mathrm{i}$ trigger}

Per a l'estudi d'estats finals amb dos taus s'ha disenyat una col.lecció de diferents triggers. Per una banda aquests triggers han de seleccionar succesos amb un leptó, electró o muó, i, a més a més, una traça aïllada al succés ("lepton+track"), que permeten identificar processos en els quals un tau es desintegra leptònicament i l'altre hadrònicament. Per una altra, cal trobar successos en els quals un tau es desintegra en un electró i l'altre en un muó ("SUSY dilepton"). L'eficiència del trigger utilitzat a aquesta cerca ha estat analitzada i calculada detalladament com mostra el capítol 5.

Les mostres de dades utilitzades per a aquesta tesi són les corresponents a aquestes dues famílies de triggers. Les dades considerades van ser adquierides durant els primers anys de l'experiment amb una una lluminositat integrada d'aproximadament $1.8 \mathrm{fb}^{-1}$.

Les diferents contribucions del MS a l'estat final s'han estimat o bé amb dades reals, quan és factible, o bé amb mostres de simulació. A més a més, aquestes mostres de simulació són necessàries per a avaluar l'eficiència de reconstrucció i identificació així com l'acceptància global.

En el cas d'aquest anàlisi, la contribució dels processos electrofebles $\left(\mathrm{Z}, \mathrm{W}^{ \pm}\right.$, dibonsons, ... ) i de producció de parelles $\mathrm{t} \overline{\mathrm{t}}$ han estat estimades amb mostres de simulació generades amb PYTHIA. Igualment, l'acceptància per a possibles successos de nova física també s'ha avaluat amb mostres de simulació, secció 4.2. Per contra, l'eficiència del trigger i els processos lligats a la cromodinàmica quàntica s'han calculat amb dades recollides en mostres de jets o leptons d'alt $p_{\mathrm{T}}$.

\section{A.4 Identificació de partícules i selecció de succesos}

La reconstrucció i identificació de leptons es troba molt ben entesa a CDF. Els electrons es reconstrueixen a partir de dipòsits energètics electromagnètics al calorímetre que coincideixen amb una traça. La identificació d'un electró amb suficient puresa es duu a terme exigint que l'electró reconstruït acomplisca els talls relacionats a la secció 6.1 . 
Els muons són reconstruïts combinant traces amb matrius a l'espectròmetre i requerint un dipòsit d'energia al calorímetre compatible amb una partícula mínimament ionitzant. La llista completa de talls aplicats en la identificació de muons es troba en la secció 6.2 .

La reconstrucció de taus hadrònics és més sofisticada. Es basa en intentar reconstruir tots el mesons als quals es desintegra el leptó tau. Per una banda, els mesons carregats són identificats mitjançant traces i dipòsits d'energia preferentment hadrònica i s'assumeix que es tracta de pions carregats, $\pi^{ \pm}$. Complementàriament, l'energia dels mesons neutres es calcula amb el calorímetre i es localitzen mitjancant el detector de màxim de cascada, sempre considerant que es tracta de pions neutres, $\pi^{0}$, secció 6.3. Posteriorment s'apliquen algunes correccions per a minvar l'efecte d'aquestes assumcions.

Com què a l'estat final abunden els neutrins, l'energia transversa perduda té una importància singular a aquest anàlisi. Es calcula respecte al vertex de la col.lisió principal i es corregeix per l'escala d'energia dels jets, muons, electrons i taus.

Finalment, la selecció final de successos es basa en la identificació dels porductes finals de les desintegracions de dos leptons tau. La significància de la cerca depén no solament de la eficiència d'identificació de succesos interesants, sinó que també de la reducció de les contribucions de successos generats per altres processos. Per aquesta raó, s'apliquen talls per a reduir les contribucions de succesos poc interessants. Per exemple, el tall en $\zeta$, secció 7.2.2 pràcticament elimina la contaminació de succesos del tipus $\mathrm{W}^{ \pm}+$jets, mentre que el tall en $\mathrm{H}_{T}$ redueix molt significativament els processos de tipus QCD, secció 7.2.3.

Un dels fons mes difícils de controlar i reduir es el causat per la confusió de jets i taus. Al Tevatron es produeixen moltíssims jets que, encara que amb poca probablitat, fluctuen a signatures molt semblants a les que produeix un tau. Per a entendre aquesta font de successos de fons s'ha emprat un sistema de determinació de la taxa de falsificació de jets a taus. La taxa es calcula en una mostra de dades independent i s'aplica després a la mostra final, secció 7.1. 


\section{A.5 Resultats}

Una vegada demostrat que les contribucions del MS han estat entenses amb precisió, l'estratègia d'extracció de senyal entra en joc. La variable escollida per a discriminar una possible senyal del bosons de Higgs de la MSMS és la "massa visible". Reconstrüts el quadrivectors dels productes visibles dels taus, el leptó en en cas de les desintegracions leptóniques o els mesons per a les desintegracions hadròniques, es computa el quadrivector suma d'aquestos productes i l'energia tranversa perduda. L'anomenada "massa visible" correspon a la massa associada a aquest quadrivector suma.

A sovint les cerques de noves partícules s'han fet amb experiments de contatge. Tanmateix en aquest cas s'ha triat una variables discriminatòria per a poder fer un ajust de les expectatives a les dades adquirides. El procediment consisteix en determinar les contribucions del cada fons del MS a la distribució final esperada. Alhora de fer l'ajust, cada contribució fluctua segons els errors sistemàtics estimats per a cada tipus de procés. Aquestos errors sistemàtics estan associats a les diferents indeterminacions experimentals, com l'incertesa en la mesura de la lluminositat, o teòriques, com els errors de truncament a la suma de sèries pertorbatives.

Es defineix una versemblança que es minimitza escanetjant en la secció eficaç de les possibles contribucions de la senyal buscada, enregistrant la secció eficaç de senyal per a la que s'integrant un $95 \%$ de l'àrea de l'estadístic, en el nostre cas $-\log (\mathcal{L}$. Es repeteix aquesta operació per a un nombre suficient de pseudoexperiments, i es defineix el límit esperat com la mediana de la distribució, i s'assignen bandes $+1 \sigma \mathrm{i}-1 \sigma$ als punts on s'integra el $68 \%$ de la probabilitat. Aquest procediment bayesià s'aplica una vegada més a les dades, que ens donaran el límit observat.

Si en l'escaneig en secció eficaç la versemblanç no prefereix el valor nul, pot haver-hi un indici de senyal. En aquest cas caldria calcular la significància de l'excés.

Realitzada la cerca, no s'ha trobat cap excés significatiu sobre les previsions del MS. El resultat de la cerca es un límit d'exclusió a un nivell de confiança del 95\%, de la secció eficaç de producció multiplicada per la fracció d'embrancament. Per a interpretar aquest límit en el marc del MSMS, ha estat traduit al pla tan $\beta$ vs. $\mathrm{m}_{\mathrm{A}^{0}}$, 
figura 8.12, segons els escenaris habituals.

\section{A.6 Conclusions}

Aquest anàlisi es fonamenta principalment en dos pilars: la solidesa de la reconstrucció i identificació de taus amb CDF i la robustesa de la predicció de les contribucions del MS a l'estat final estudiat. La fiabilitat de la reconstrucció de taus ha quedat justificada per les mesures accesòries amb taus i altres leptons (com les seccions eficaces de processos com $\mathrm{Z} \rightarrow \tau \tau, \mathrm{Z} \rightarrow$ e e i $\mathrm{Z} \rightarrow \mu \mu$ ), mentre que la predicció de les contribuions del MS s'ha comprobat en les diverses regions de control.

Tot i que el resultat de la bùsqueda és negatiu, limita l'espai de paràmetres possibles per a la MSMS més que cap altre experiment (incloent els resultats de DØ). Amb la vista posada a l'LHC, el proper col.lisionador d'hadrons, aquest anàlisi suposa una referència molt valuosa.

La quantitat de dades adquirides pels experiments del Tevatron i el progrés en les tècniques experiments utilitzades han fet avançar la sesibilitat d'una forma significativa. L'anàlisi expossat en aquesta tesi ha supossat un pas important en el coneixement de la física fonamental. La inclusió d'aquest resultat en el Particle Data Group Review of Particle Physics fa palessa la qualitat i la relevància que la comunitat de física de partícules reconeix en aquest treball. 


\section{Bibliography}

[1] Mary K. Gaillard, Paul D. Grannis, and Frank J. Sciulli. The standard model of particle physics. Rev. Mod. Phys., 71:S96-S111, 1999.

[2] A.D. Martin F. Halzen. Quarks and Leptons: An Introductory Course in Modern Particle Physics. Wiley Text Books, 1984.

[3] David Griffiths. Introduction to Elementary Particles. Wiley, 1987.

[4] W. M. Yao et al. Review of particle physics. J. Phys., G33:1-1232, 2006.

[5] CDF and D $\varnothing$ Collaborations. A combination of cdf and DØ results on the mass of the top quark. hep-ex/0703034, 2007.

[6] R. P. Feynman. Mathematical formulation of the quantum theory of electromagnetic interaction. Phys. Rev., 80:440-457, 1950.

[7] R. Keith Ellis, W. James Stirling, and B. R. Webber. Qcd and collider physics. Camb. Monogr. Part. Phys. Nucl. Phys. Cosmol., 8:1-435, 1996.

[8] G. Dissertori, I. G. Knowles, and M. Schmelling. High energy experiments and theory. Oxford, UK: Clarendon (2003) 538 p.

[9] John C. Collins. The problem of scales: Renormalization and all that. 1995.

[10] A. D. Martin, W. J. Stirling, R. S. Thorne, and G. Watt. Update of parton distributions at nnlo. Phys. Lett., B652:292-299, 2007. MRST Webpage, http://durpdg.dur.ac.uk/hepdata/mrs.html. 
[11] H. L. Lai et al. Improved parton distributions from global analysis of recent deep inelastic scattering and inclusive jet data. Phys. Rev., D55:1280-1296, 1997. CTEQ Webpage, http://www.phys.psu.edu/ cteq/.

[12] D. Stump et al. Uncertainties of predictions from parton distribution functions. i: The lagrange multiplier method. Phys. Rev., D65:014012, 2002.

[13] J. Pumplin et al. Uncertainties of predictions from parton distribution functions. ii: The hessian method. Phys. Rev., D65:014013, 2002.

[14] E. Fermi. An attempt of a theory of beta radiation. 1. Z. Phys., 88:161-177, 1934.

[15] S. L. Glashow. Partial symmetries of weak interactions. Nucl. Phys., 22:579$588,1961$.

[16] Abdus Salam and John Clive Ward. Electromagnetic and weak interactions. Phys. Lett., 13:168-171, 1964.

[17] Steven Weinberg. A model of leptons. Phys. Rev. Lett., 19:1264-1266, 1967.

[18] L. D. Faddeev and V. N. Popov. Feynman diagrams for the yang-mills field. Phys. Lett., B25:29-30, 1967.

[19] G. Arnison et al. Experimental observation of lepton pairs of invariant mass around 95-gev/c**2 at the cern sps collider. Phys. Lett., B126:398-410, 1983.

[20] G. Arnison et al. Experimental observation of isolated large transverse energy electrons with associated missing energy at $\mathrm{s}^{* *}(1 / 2)=540$-Gev. Phys. Lett., B122:103-116, 1983.

[21] H. Georgi, Helen R. Quinn, and Steven Weinberg. Hierarchy of interactions in unified gauge theories. Phys. Rev. Lett., 33:451-454, 1974.

[22] Leonard Susskind. Dynamics of spontaneous symmetry breaking in the weinberg- salam theory. Phys. Rev., D20:2619-2625, 1979.

[23] Stephen P. Martin. A supersymmetry primer. 1997.

[24] Hitoshi Murayama. Supersymmetry phenomenology. 2000. 
[25] Rudolf Haag, Jan T. Lopuszanski, and Martin Sohnius. All possible generators of supersymmetries of the s matrix. Nucl. Phys., B88:257, 1975.

[26] Robert Nyden Hill and Edward H. Kerner. Unique canonical representation of the inhomogeneous lorentz group in relativistic particle dynamics. Phys. Rev. Lett., 17:1156, 1966.

[27] Joseph Polchinski and Leonard Susskind. Breaking of supersymmetry at intermediate-energy. Phys. Rev., D26:3661, 1982.

[28] Edward Witten. Dynamical breaking of supersymmetry. Nucl. Phys., B188:513, 1981.

[29] Romesh K. Kaul. Gauge hierarchy in a supersymmetric model. Phys. Lett., B109:19, 1982.

[30] Ugo Amaldi, Wim de Boer, and Hermann Furstenau. Comparison of grand unified theories with electroweak and strong coupling constants measured at lep. Phys. Lett., B260:447-455, 1991.

[31] Ugo Amaldi, Wim de Boer, Paul H. Frampton, Hermann Furstenau, and James T. Liu. Consistency checks of grand unified theories. Phys. Lett., B281:374-383, 1992.

[32] Paul Langacker and Ming-xing Luo. Implications of precision electroweak experiments for $\mathrm{m}(\mathrm{t})$, rho(0), $\sin ^{* *} 2$-theta(w) and grand unification. Phys. Rev., D44:817-822, 1991.

[33] John R. Ellis, S. Kelley, and Dimitri V. Nanopoulos. Probing the desert using gauge coupling unification. Phys. Lett., B260:131-137, 1991.

[34] F. Gliozzi, Joel Scherk, and David I. Olive. Supersymmetry, supergravity theories and the dual spinor model. Nucl. Phys., B122:253-290, 1977.

[35] S. Heinemeyer, W. Hollik, D. Stockinger, A. M. Weber, and G. Weiglein. Precise prediction for $\mathrm{m}(\mathrm{w})$ in the mssm. JHEP, 08:052, 2006.

[36] H. E. Haber, (ed. ) Harvey, Jeffrey A., and (ed. ) Polchinski, J. Recent directions in particle theory: From superstrings and black holes to the standard model. proceedings, theoretical advanced study institute in elementary 
particle physics, boulder, usa, june 1-26, 1992. pages 589-686. Singapore, Singapore: World Scientific (1993) 827 p.

[37] David J. Gross and R. Jackiw. Effect of anomalies on quasirenormalizable theories. Phys. Rev., D6:477-493, 1972.

[38] C. Bouchiat, J. Iliopoulos, and P. Meyer. An anomaly free version of weinberg's model. Phys. Lett., B38:519-523, 1972.

[39] Luis Alvarez-Gaume and Edward Witten. Gravitational anomalies. Nucl. Phys., B234:269, 1984.

[40] John F. Gunion, Howard E. Haber, Gordon L. Kane, and Sally Dawson. The higgs hunter's guide. SCIPP-89/13.

[41] S. Dawson. The mssm and why it works. 1997.

[42] Savas Dimopoulos and Howard Georgi. Softly broken supersymmetry and su(5). Nucl. Phys., B193:150, 1981.

[43] N. Sakai. Naturalness in supersymmetric guts. Zeit. Phys., C11:153, 1981.

[44] P. Fayet. Spontaneously broken supersymmetric theories of weak, electromagnetic and strong interactions. Phys. Lett., B69:489, 1977.

[45] Pierre Fayet. Relations between the masses of the superpartners of leptons and quarks, the goldstino couplings and the neutral currents. Phys. Lett., B84:416, 1979.

[46] Steven Weinberg. Supersymmetry at ordinary energies. 1. masses and conservation laws. Phys. Rev., D26:287, 1982.

[47] N. Sakai and Tsutomu Yanagida. Proton decay in a class of supersymmetric grand unified models. Nucl. Phys., B197:533, 1982.

[48] Savas Dimopoulos, Stuart Raby, and Frank Wilczek. Proton decay in supersymmetric models. Phys. Lett., B112:133, 1982.

[49] John R. Ellis, Dimitri V. Nanopoulos, and Serge Rudaz. Guts 3: Susy guts 2. Nucl. Phys., B202:43, 1982. 
[50] Glennys R. Farrar and Pierre Fayet. Phenomenology of the production, decay, and detection of new hadronic states associated with supersymmetry. Phys. Lett., B76:575-579, 1978.

[51] F. Zwirner. Observable delta $\mathrm{b}=2$ transitions without nucleon decay in a minimal supersymmetric extension of the standard model. Phys. Lett., B132:103-106, 1983.

[52] Lawrence J. Hall and Mahiko Suzuki. Explicit r-parity breaking in supersymmetric models. Nucl. Phys., B231:419, 1984.

[53] John R. Ellis, G. Gelmini, C. Jarlskog, Graham G. Ross, and J. W. F. Valle. Phenomenology of supersymmetry with broken r-parity. Phys. Lett., B150:142, 1985.

[54] Graham G. Ross and J. W. F. Valle. Supersymmetric models without rparity. Phys. Lett., B151:375, 1985.

[55] Sally Dawson. R-parity breaking in supersymmetric theories. Nucl. Phys., B261:297, 1985.

[56] Savas Dimopoulos and Lawrence J. Hall. Lepton and baryon number violating collider signatures from supersymmetry. Phys. Lett., B207:210, 1988.

[57] Carl E. Carlson, Probir Roy, and Marc Sher. New bounds on r-parity violating couplings. Phys. Lett., B357:99-104, 1995.

[58] Gautam Bhattacharyya. R-parity-violating supersymmetric yukawa couplings: A mini- review. Nucl. Phys. Proc. Suppl., 52A:83-88, 1997.

[59] John F. Gunion, Howard E. Haber, Gordon L. Kane, and Sally Dawson. Errata for the Higgs hunter's guide. 1992.

[60] Marcela S. Carena and Joseph D. Lykken. Physics at Run II: Supersymmetry / Higgs Workshop. Prepared for Physics at Run II: Workshop on Supersymmetry / Higgs: 1st General Meeting, Batavia, Illinois, 14-16 May 1998.

[61] Fermilab Beam Division. http://www-bd.fnal.gov. 
[62] Fermilab Beam Division. Run II Handbook. http://wwwbd.fnal.gov/runII/index.html.

[63] R. Blair et al. The cdf-ii detector: Technical design report. Technical report, FNAL, 1996. FERMILAB-PUB-96/390-E.

[64] A. Sill. Cdf run ii silicon tracking projects. Nucl. Instrum. Meth., A447:1-8, 2000.

[65] Anthony Allen Affolder et al. Cdf central outer tracker. Nucl. Instrum. Meth., A526:249-299, 2004.

[66] CDF Tracking Group. see http://wwwcdf.fnal.gov/internal/detectors/parameters.html and http://wwwcdf.fnal.gov/cdfsim/validation/cot/cotVal.html.

[67] C. Paus et al. Design and performance tests of the CDF time-of-flight system. Nucl. Instrum. Meth., A461:579-581, 2001.

[68] C. Grozis et al. A time-of-flight detector for cdf. Int. J. Mod. Phys., A16S1C:1119-1121, 2001.

[69] C. Grozis et al. The time of flight detector at cdf. Nucl. Phys. Proc. Suppl., 93:344-347, 2001.

[70] L. Balka et al. The cdf central electromagnetic calorimeter. Nucl. Instrum. Meth., A267:272, 1988.

[71] S. Bertolucci et al. The cdf central and endwall hadron calorimeter. Nucl. Instrum. Meth., A267:301, 1988.

[72] Lawrence Nodulman. Hybrid shower counters for CDF. Nucl. Instr. Meth., 176:345, 1980.

[73] L. Nodulman et al. Central shower counter prototype for the Fermilab Collider Detector Facility. Nucl. Instr. Meth., 204:351, 1983.

[74] A Anastassov. Non-isolated $\pi^{0} / \gamma$ reconstruction. CDF $/ 6688,2003$.

[75] L Nodulman. Ces strp/wire ratio tuning in run ii. CDF/MEMO/ELECTRON/CDFR/8211, 2006. 
[76] M. G. Albrow et al. The cdf plug upgrade electromagnetic calorimeter: Test beam results. Nucl. Instrum. Meth., A480:524-546, 2002.

[77] C. M. Ginsburg. Cdf run 2 muon system. Eur. Phys. J., C33:s1002-s1004, 2004.

[78] D. Acosta et al. The cdf cherenkov luminosity monitor. Nucl. Instrum. Meth., A461:540-544, 2001.

[79] D. Acosta et al. The performance of the cdf luminosity monitor. Nucl. Instrum. Meth., A494:57-62, 2002.

[80] F. Abe et al. Measurement of the $\bar{p} p$ total cross-section at $\sqrt{s}=546 \mathrm{gev}$ and 1800-gev. Phys. Rev., D50:5550-5561, 1994.

[81] S. Klimenko, J. Konigsberg, and Tony M. Liss. Averaging of the inelastic cross sections measured by the cdf and the e 811 experiments. FERMILABFN-0741.

[82] S. Jundariani et al. Luminosity uncertainty for run 2 up until august 2004. $\mathrm{CDF} / \mathrm{ANA} / 7446,2005$.

[83] H.J. Frisch and P.J. Wilson. Trigger tower organization and summing in $\eta-\phi$ space for run ii and beyond. CDF/DOC/TRIGGER/CDFR/2045, 2001.

[84] The xft system webpage. http://www.physics.ohiostate.edu/ hughes/xft/.

[85] The xtrp webpage. http://web.hep.uiuc.edu/engin/cdf/xtrp/.

[86] I. Vila. Performance and first physics results of the svt trigger at cdf ii. 2003.

[87] The Trigger and Datasets Working Group. Run ii trigger table and datasets plan. CDF/PHYS/TRIGGER/CDFR/4718, 2001.

[88] Amit Lath Sunil Somalwar Sourabh Dube, John Zhou. Searches for charginoneutralino production in msugra model in a di-electron + track channel. $\mathrm{CDF} / 8445,2006$. 
[89] John Strologas Michael Gold, Vladimir Rekovic. Search for charginoneutralino production in the inclusive low-pt dimuon+lepton channel with $1 \mathrm{fb}-1 . \mathrm{CDF} / 8479,2006$.

[90] Torbjorn Sjostrand, Leif Lonnblad, and Stephen Mrenna. PYTHIA 6.2: Physics and manual. 2001.

[91] R. Brun et al. GEANT 3 manual, CERN Program Library Long Writeup. 1994.

[92] E. Gerchtein and M. Paulini. CDF detector simulation framework and performance. 2003.

[93] S. Jadach, Z. Was, R. Decker, and Johann H. Kuhn. The tau decay library TAUOLA: Version 2.4. Comput. Phys. Commun., 76:361-380, 1993.

[94] Sunil Somalwar Sourabh Dube, John Zhou. Medium et electron identification efficiency and scale-factors. CDF/8321, 2006.

[95] D.Bortoletto E.Lytken, A.Canepa. Intermediate pt muon id efficiencies with the $1 \mathrm{fb}-1$ dataset. $\mathrm{CDF} / 8336,2006$.

[96] Ulysses Grundler Anyes Taffard Xiaojian Zhang. High-pt muons recommended cuts and efficiencies for summer 2006. CDF/8262, 2006.

[97] A. Abulencia et al. Search for neutral MSSM Higgs bosons decaying to tau pairs in $p \bar{p}$ collisions at $\sqrt{s}=1.96 \mathrm{TeV}$. Phys. Rev. Lett., 96:011802, 2006.

[98] Darin E. Acosta et al. Search for new physics using high mass tau pairs from $1.96 \mathrm{TeV} p \bar{p}$ collisions. Phys. Rev. Lett., 95:131801, 2005.

[99] M. Goncharov T. Kamon V. Khotilovich R. Lander T. Ogawa C. Pagliarone F. Ratnikov A. Safonov J.R. Smith E. Vataga S. Baroiant, M. Chertok. Energy measurement for hadronic taus. CDF/6654, 2003.

[100] A. Abulencia. Measurement of sigma p anti-p -i Z . Br $(\mathrm{Z}-i$ 2tau $)$ in $\mathrm{p}$ anti-p collisions at $\mathrm{s}^{* *}(1 / 2)=1.96 \mathrm{TeV}$. Phys. Rev., D75:092004, 2007.

[101] John Conway Dongwook Jang Amit Lath Anton Anastassov, Cristobal Cuenca. Search for neutral mssm higgs boson(s) in the tau tau decay channel. CDF/8639, 2007. 
[102] Marcela S. Carena, S. Heinemeyer, C. E. M. Wagner, and G. Weiglein. Suggestions for benchmark scenarios for MSSM Higgs boson searches at hadron colliders. Eur. Phys. J., C26:601-607, 2003.

[103] Marcela S. Carena, S. Heinemeyer, C. E. M. Wagner, and G. Weiglein. Suggestions for improved benchmark scenarios for Higgs- boson searches at LEP2. 1999.

[104] F. Maltoni, Z. Sullivan, and S. Willenbrock. Higgs-boson production via bottom-quark fusion. Phys. Rev., D67:093005, 2003.

[105] Robert V. Harlander and William B. Kilgore. Higgs boson production in bottom quark fusion at next-to- next-to-leading order. Phys. Rev., D68:013001, 2003.

[106] M. Frank et al. The Higgs boson masses and mixings of the complex MSSM in the Feynman-diagrammatic approach. JHEP, 02:047, 2007.

[107] Michael Spira. HIGLU and HDECAY: Programs for Higgs boson production at the LHC and Higgs boson decay widths. Nucl. Instrum. Meth., A389:357$360,1997$.

[108] Michael Spira. HIGLU: A Program for the Calculation of the Total Higgs Production Cross Section at Hadron Colliders via Gluon Fusion including QCD Corrections. 1995.

[109] Marcela S. Carena, S. Heinemeyer, C. E. M. Wagner, and G. Weiglein. MSSM Higgs boson searches at the Tevatron and the LHC: Impact of different benchmark scenarios. Eur. Phys. J., C45:797-814, 2006.

[110] A. Connolly. A search for supersymmetric higgs bosons in the di-tau decay mode in p-anti-p collisions at 1.8 tev. CDF/6806, 2003. 


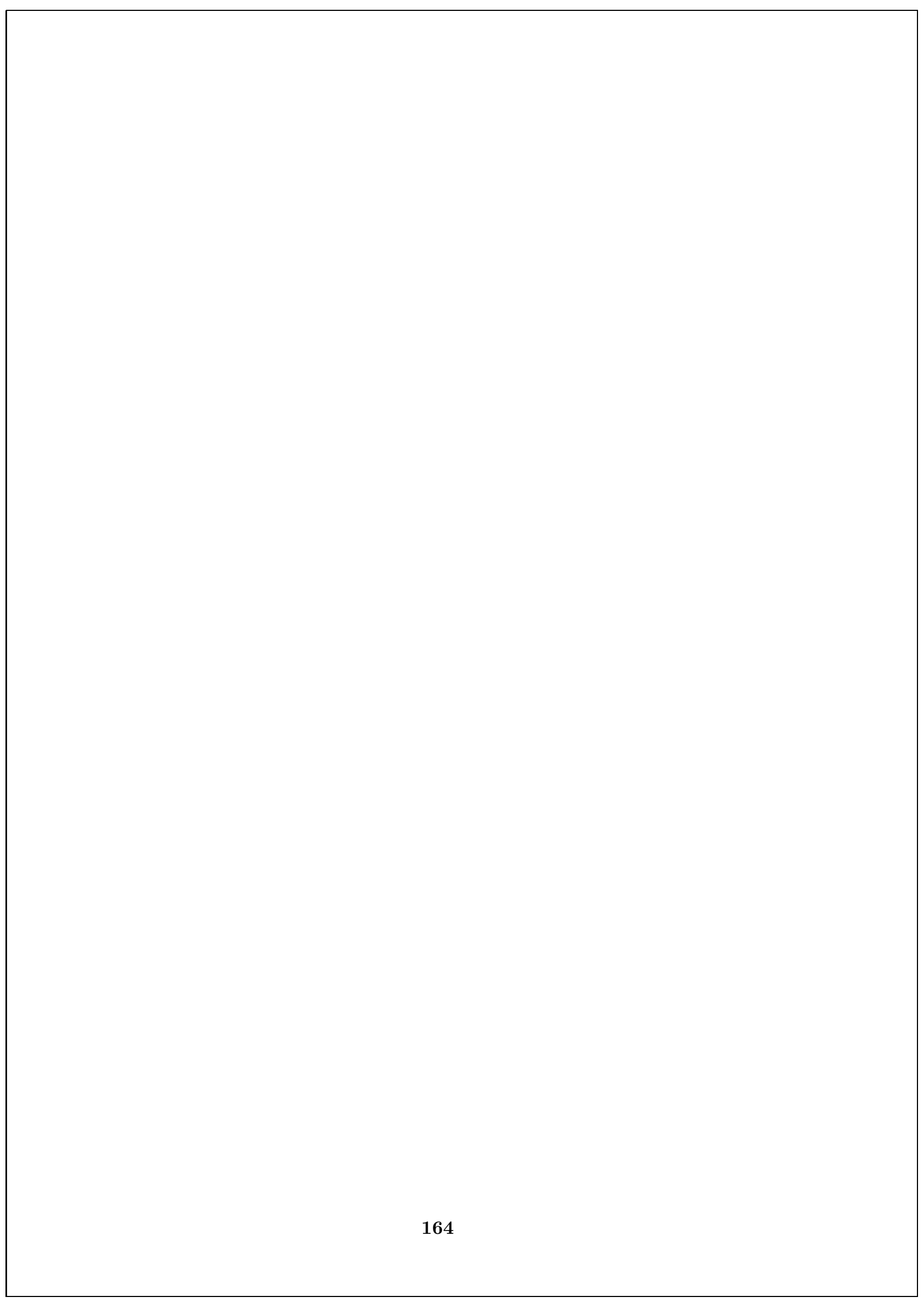




\section{List of Figures}

2.1 Basic components of the Standard Model . . . . . . . . . . . . 6

2.2 The value of the running coupling constant, $\alpha_{S}$, as a function of the energy scale E . . . . . . . . . . . . . . . . . 10

2.3 Uncertainty on gluon and u-quark PDFs . . . . . . . . . . . 14

2.4 The minimum of the Higgs potential occurs at $-\mu^{2} /(2 \lambda)$, not at zero 19

$2.5 M_{W}$ as a function of $m_{t}$ as predicted by the SM. . . . . . . 26

2.6 MSSM neutral Higgs bosons mass spectrum . . . . . . . . . . . 35

2.7 Main MSSM pseudoscalar Higgs boson production diagrams . . . . 36

2.8 Standard Model Higgs boson production cross sections at the Teva-

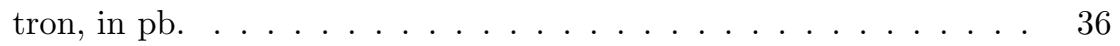

2.9 Pseudoscalar MSSM Higgs boson production cross sections at the Tevatron, in $\mathrm{pb}$, for $\tan \beta=30 \ldots \ldots \ldots \ldots$

2.10 Pseudoscalar MSSM Higgs boson branching ratios, for $\tan \beta=6$ and $\tan \beta=30 \ldots \ldots \ldots \ldots \ldots \ldots \ldots \ldots$

3.1 The Tevatron Collider Chain at Fermilab . . . . . . . . . . . . 40

3.2 Tevatron Collider Run II Integrated Luminosity . . . . . . . . . . . 42 
3.3 Tevatron Collider Run II Peak Luminosity . . . . . . . . . . . . . . 43

3.4 Isometric view of the CDF Run II detector . . . . . . . . . . 44

$3.5 r \times \eta$ side view of the CDF Run II detector $\ldots \ldots \ldots \ldots 4$

3.6 Layout of wire planes on a COT endplate . . . . . . . . . . 46

3.7 Layout of wires in a COT supercell . . . . . . . . . . . . . . 47

3.8 The CDF II tracker layout showing the different subdetector systems 48

3.9 CDF calorimeter system . . . . . . . . . . . . . . . . . . . 49

3.10 Invariant mass of $\pi^{0}$ candidates, reconstructed from two resolved

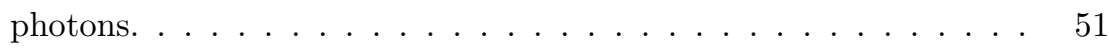

3.11 CDF muon system . . . . . . . . . . . . . . . 54

3.12 CLC tube schematic view . . . . . . . . . . . 55

3.13 Block diagram showing the global trigger and DAQ systems at CDF II 58

3.14 Block diagram showing the Level 1 and Level 2 trigger system . . . 62

4.1 Comparison of the highest- $p_{\mathrm{T}}$ b-quark produced in the hard scattering in different processes in PYтHIA $6.216 \ldots \ldots \ldots$

5.1 XFT track finding efficiency for tracks that cross the $Z=0$ plane. $\quad 74$

5.2 XFT track finding efficiency for tracks that don't cross the $Z=0$

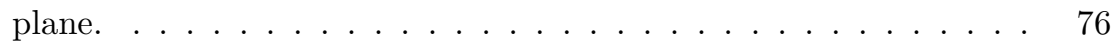

5.3 Comparison of the efficiency in Jet20 data and QCD 18 MC. . . . 77

5.4 L3 efficiency parameterized in terms of the number of primary verteces for the old trigger. . . . . . . . . . . . . . . 79

5.5 L3 efficiency parameterized in terms of the number of primary verteces for the new trigger. . . . . . . . . . 80 
5.6 L3 "migration" efficiency for the "old trigger", parameterized in terms of $\Delta \theta_{\text {min }} \ldots \ldots \ldots \ldots \ldots \ldots \ldots \ldots$

5.7 L3 "migration" efficiency for the "new trigger", parameterized in

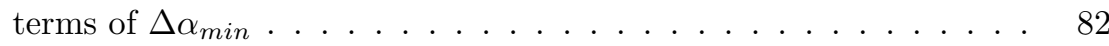

6.1 Invariant mass of the selected electron pairs ( $1 \mathrm{fb}-1$ sample $). . \quad 87$

6.2 Data/MC scale factor for track isolation of electrons and muons plotted as a function muon $p_{T}\left(\right.$ electron $\left.E_{T}\right) \ldots \ldots \ldots$

6.3 Invariant mass of selected muon pairs ( $1 \mathrm{fb}-1$ sample) . . . . . . . 89

6.4 Comparison of the efficiency of the cuts in data and MC using $Z$ events $\left(1.8 \mathrm{fb}^{-1}\right.$ sample $) \ldots \ldots \ldots \ldots$. . . . . . . . . . . 94

6.5 Track multiplicity and $m\left(t r k s+\pi^{0}\right.$ 's) for tau candidates. . . . . 95

6.6 Distributions of tau $p_{T}$ in data and $\mathrm{MC}$ after correcting data for the observed shifts. . . . . . . . . . . . . . . . 96

7.1 Consistency checks of $j e t \rightarrow \tau$ fake predictions using the $\gamma+j e t$ sample. . . . . . . . . . . . . . . . . . . . 101

7.2 Consistency checks of $j e t \rightarrow \tau$ fake predictions using the non-isolated electron sample. . . . . . . . . . . . . . . . . . . . . . 102

7.3 Consistency checks of $j e t \rightarrow \tau$ fake predictions using the non-isolated muon sample. . . . . . . . . . . . . . . . . . . 103

7.4 Consistency checks of $j e t \rightarrow \tau$ fake predictions using region dominated by $W+j e t(s), W \rightarrow e \nu . \ldots \ldots \ldots$. . . . . . . 104

7.5 Consistency checks of jet $\rightarrow \tau$ fake predictions using region dominated by $W+j e t(s), W \rightarrow \mu \nu . \ldots \ldots \ldots \ldots$

7.6 Illustration of the definition of parameters used in the $\zeta$ cut. . . . 107

7.7 Effect of the $\zeta$ cut. . . . . . . . . . . . . . . 108 
7.8 Effect of the $\left|E_{T}^{e}\right|+\left|p_{T}^{\mu}\right|$ cut on like-sign data events and Higgs signal $\left(m_{A}=90,200 \mathrm{GeV}\right) . \ldots \ldots \ldots \ldots$

7.9 Track isolation for electrons and muons in same-sign events passing all other selection criteria. . . . . . . . . . . . . . . . . 111

7.10 Signal selection efficiencies for the three detection modes as a function of Higgs mass $\left(m_{A}\right) . \quad \ldots \ldots \ldots \ldots$

8.1 Distributions of data and predicted backgrounds for the projections on the two axes used in the "zeta-cut . . . . . . . . . . 119

8.2 Distributions for $\tau_{e} \tau_{\text {had }}$ candidate events. . . . . . . . . . . 120

8.3 Distributions for $\tau_{e} \tau_{\text {had }}$ candidate events. . . . . . . . . . . . 121

8.4 Distributions for $\tau_{\mu} \tau_{h a d}$ candidate events. . . . . . . . . . . . . 122

8.5 Distributions for $\tau_{\mu} \tau_{\text {had }}$ candidate events. . . . . . . . . . . . 123

8.6 Distributions for $\tau_{e} \tau_{\mu}$ candidate events. . . . . . . . . . . 124

8.7 Distributions for $\tau_{e} \tau_{\mu}$ candidate events. . . . . . . . . . 125

8.8 Distributions in the three channels used for signal extraction. . . . 126

8.9 Visible mass distributions for $Z$ and Higgs bosons (normalized to

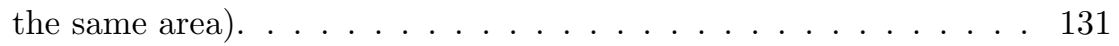

8.10 Observed and expected 95\% CL upper limits on $\sigma(p \bar{p} \rightarrow \phi+X) \times$ $B R(\phi \rightarrow \tau \tau) \ldots \ldots \ldots \ldots \ldots \ldots \ldots \ldots \ldots \ldots$

8.11 Observed distribution of $m_{v i s}$ along with a signal corresponding to $m_{A}=140 \mathrm{GeV} \ldots \ldots \ldots \ldots \ldots \ldots \ldots$

8.12 Excluded $\tan \beta$ as a function of $m_{A}$ for the "no-mixing" and $m_{h}^{\max }$ scenarios with $\mu>0$ and $\mu<0 \ldots \ldots \ldots \ldots$

8.13 Di-tau mass reconstructed with the $E_{\mathrm{T}}$ projection method. . . . . 142 
9.1 Excluded $\tan \beta$ as a function of $m_{A}$ for the 3 b-quark analysis at CDF144

9.2 Excluded $\tan \beta$ as a function of $m_{A}$ regions for LEP, DØand CDF using the $\tau \tau$ decay channel . . . . . . . . . . . . . . . . . . 144 


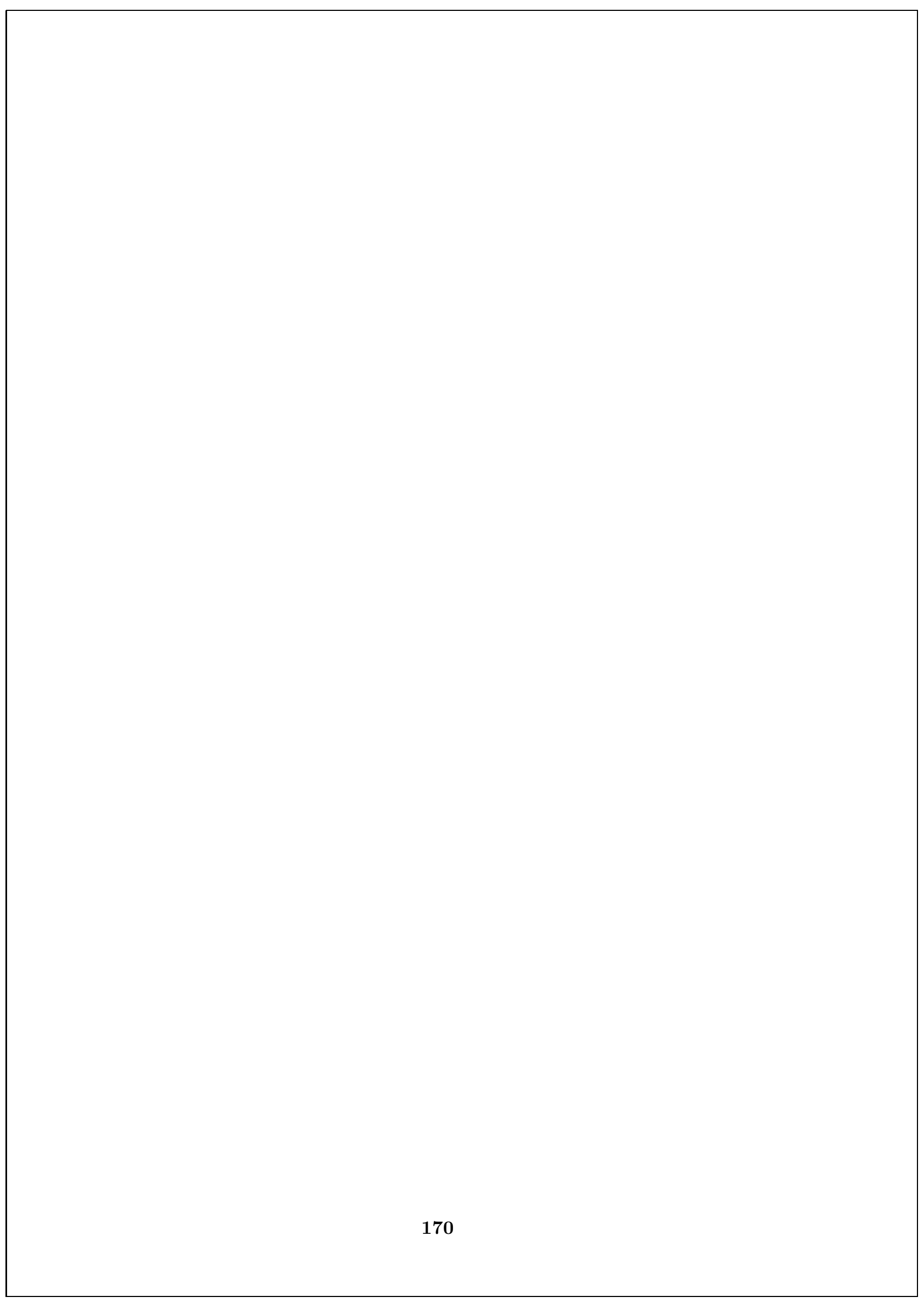




\section{List of Tables}

2.1 Fermionic sector of the SM. . . . . . . . . . . . . . 7

2.2 The gauge bosons of the Standard Model and their interactions. . 8

2.3 Superfields and particle content of the MSSM. . . . . . . . . . . . . 29

2.4 The particle content of the MSSM. . . . . . . . . . . . . . . 31

3.1 Accelerator parameters for Run II configuration . . . . . . . . . . . 42

3.2 CDF II Calorimeter subsystems and characteristics . . . . . . . . 52

5.1 "lepton plus track" trigger paths used in this analysis . . . . . . 70

5.2 L2 cluster cuts. . . . . . . . . . . . . . . . . . 71

5.3 Level 3 track cuts. . . . . . . . . . . . . . . . . 71

5.4 Isolation annulus definition, and cuts for shoulder tracks . . . . . . 71

5.5 Fitted parameters for the L2 trigger efficiency as a function of $R Z 0$. 73

5.6 Fitted parameters for the L2 trigger efficiency as a function of $\operatorname{Lr} z . \quad 75$

5.7 Results of the fits to $\Delta \theta_{\text {min }}$ and $\Delta \alpha_{\text {min }} \ldots \ldots \ldots$. . . . . . . . 83 
8.1 Predicted backgrounds and observed events in the $\tau_{e} \tau_{\mu}$ after applying all selection cuts. . . . . . . . . . . . . . . . . . 117

8.2 Predicted backgrounds and observed events in the $\tau_{e} \tau_{\text {had }}$ and $\tau_{\mu} \tau_{\text {had }}$ after applying all selection cuts. . . . . . . . . . . . 118

8.3 Systematic uncertainties by source. . . . . . . . . . . . . . . . . 129

8.4 Expected and observed 95\% CL limits on the Higgs signal cross

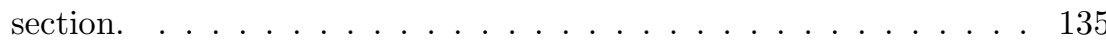




\section{Glossary}

COT Central Outer Tracker

L00 Layer 00

SVXII Silicon Vertex Detector

ISL Intermediate Silicon Layer

CEM Central Electromagnetic Calorimeter

CHA Central Hadronic Calorimeter

WHA Endwall Hadron Calorimeter

CES Shower Maximum Detector

PEM Electromagnetic Plug Calorimeter

PHA Hadronic Plug Calorimeter

PES Plug Shower Maximum

CPR Central Preradiator

PPR Plug Preradiator

CMU Central Muon Detector

CMP Central Muon Upgrade Detector

CMX Central Muon Extension Detector 
IMU Intermediate Muon Detector

BMU Barrel Chambers

CSP Central Muon Scintillator Upgrade

CSX Central Muon Extension Scintillator

BSU Barrel Scintillator Upgrade

TSU Toroid Scintillator Upgrade

CLC Čerenkov Luminosity Counters

XFT eXtremely Fast Tracker

XTRP Extrapolation Module

XCES Shower Maximum Locator

SVT Silicon Vertex Tracker 